\title{
The Influence of Geothermal Sources on Deep Ocean Temperature, Salinity, and Flow Fields \\ by
}

\author{
Kevin George Speer \\ B.S. Physics, B.S. Math \\ University of California at Santa Barbara \\ 1982 \\ SUBMITTED IN PARTIAL FULFILLMENT OF THE \\ REQUIREMENTS OF THE DEGREE OF \\ DOCTOR OF PHILOSOPHY \\ at the \\ WOODS HOLE OCEANOGRAPHIC INSTITUTION \\ and the

\section{MASSACHUSETTS INSTITUTE OF TECHNOLOGY}

May 1988

(C)Kevin G. Speer

The author hereby grants to MIT and WHOI permission to reproduce and distribute copies of this thesis document in whole or in part.

Signature of the author

Joint Program in Oceanogray.

Massachusetts Institute of Technology -

Woods Hole Oceanographic Institution, May 1988

Certified by

Terrence M. Joyce

Thesis Supervisor

Accepted by

Chairman, Jofint Committee for Physical Oceanography,

Massachusetts Institute of Technology -

Woods Hole Oceanographic Institution 


\title{
The Influence of Geothermal Sources on Deep Ocean Temperature, Salinity, and Flow Fields
}

by

\section{Kevin George Speer}

Submitted to the Massachusetts Institute of Technology-Woods Hole Oceanographic Institution Joint Program in May 1988 in partial fulfillment of the requirements for the degree of Doctor of Philosophy.

\begin{abstract}
This thesis is a study of the effect of geothermal sources on the deep circulation, temperature and salinity fields. In Chapter 1 background material is given on the strength and distribution of geothermal heating. In Chapter 2 evidence for the influence of a hydrothermal system in the rift valley of the Mid-Atlantic Ridge on nearby property fields and a model of the flow around such a heat source are presented, with an analysis of a larger-scale effect. Results of an analytical model for a heat source on a $\beta$-plane in Chapter 3 show how the response far from the source can have a structure different from the forcing because of its dependence on two parameters: a Peclet number (the ratio of horizontal advection and vertical diffusion), and a Froude-number-like parameter (the ratio of long wave phase speed to background flow speed) which control the relative amount of damping and advection of different vertical scales. The solutions emphasize the different behavior of a dynamical field like temperature compared to tracers introduced at the source. These ideas are useful for interpreting more complicated solutions from a numerical model presented in the final chapter.
\end{abstract}

Thesis Supervisor: Dr. Terrence M. Joyce

Title:

Senior Scientist

Woods Hole Oceanographic Institution 


\section{ACKNOWLEDGEMENTS}

I thank my advisor, Terry Joyce, for his support and guidance throughout this work and at the crucial moments which arise from time to time during a thesis project.

The assistance of the rest of my thesis committee, Paola Rizzoli, Mike McCartney, Nelson Hogg, Joe Pedlosky, and especially Bruce Warren (the bounds of his patience were explored once again) through several versions of this thesis was crucial to its succesful completion.

Special thanks go to Peter Rona for sharing his CTD data from the TAG site. Special thanks also go to Rick Salmon for sharing his numerical model, spending his time, and for his enthusiasm. Lorraine Barbour's expert drafting was a blessing, and I toast Ruth Gorski for her professional programming help. Sophie Wacongne has helped in many ways, which I well appreciate.

The WHOI Friends of Vents group, founded by Joe Cann, Sarah Little, Cindy L. Van Dover, Andy Trivett, and myself, gave me additional motivation for the plume studies.

Everything will be all right once you know something-RXH.

This study was supported by the National Science Foundation grants OCE8515642 to T. Joyce and OCE82-13967 to B. Warren, and by the WHOI-MIT Joint Program Ocean Ventures Fund. 


\section{Contents}

1 Geothermal Forcing 6

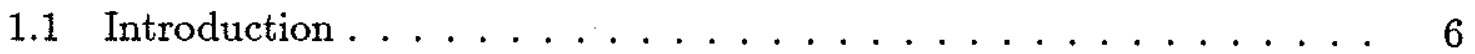

1.2 Origin of Geothermal Sources at Ocean Ridge Crests . . . . . . . 7

1.3 Regional Distribution and Strength . . . . . . . . . . . . . . 10

1.4 Related Studies and This Work . . . . . . . . . . . . . . . 14

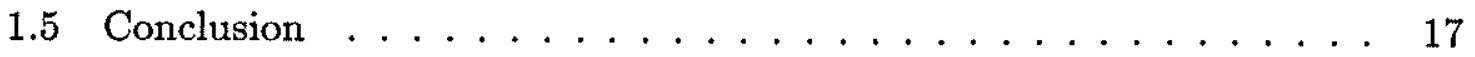

2 The Evidence for the Influence of Geothermal Heating on the Temperature and Salinity Fields in the Tropical Atlantic. 18

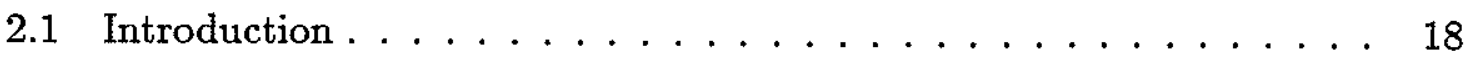

2.2 Data, Methods . . . . . . . . . . . . . . . 19

2.3 Near Field Observations . . . . . . . . . . . . . . . . . . 22

2.4 A turbulent plume model and comparison to observations . . . . . . 31

2.4 .1 Atlantic case . . . . . . . . . . . . . 32

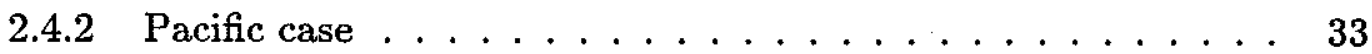

2.5 Discussion . . . . . . . . . . . . . . . . 36

2.6 Horizontal Flow: a 2-d Forced Baroclinic Vortex . . . . . . . . . 38

2.7 Large-scale $\theta-S$ anomalies above the Mid-Atlantic Ridge . . . . . 43

2.8 Summary . . . . . . . . . . . . . . . . . 53

3 The Large-scale Influence of a Heat Source 63

3.1 Introduction . . . . . . . . . . . . . . . 63

3.2 Standard Model . . . . . . . . . . . . . . . . . . . 65

3.3 Solutions $\ldots \ldots \ldots \ldots \ldots \ldots$

3.3.1 Solution with application to the South Pacific . . . . . . . 73

3.4 Extensions of the Standard Model . . . . . . . . . . . . 76

3.4.1 Increased vertical resolution $\ldots \ldots \ldots \ldots \ldots$

3.4.2 Mediterranean source example . . . . . . . . . . 77

3.4.3 Background Vertical Shear . . . . . . . . . . . . . 82

3.4 .4 Nonlinearity . . . . . . . . . . . . . . . . . . . . 88

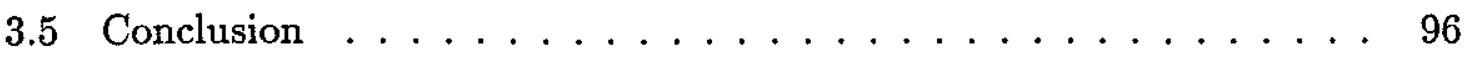


4 A Numerical Simulation of Geothermal Heating 98

4.1 Introduction . . . . . . . . . . . . . . . . . 98

4.2 Geometry, Equations, and Forcing . . . . . . . . . . . . . 99

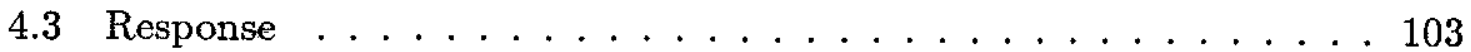

4.3.1 An example of mode separation and nonlinear correction field. 109

4.3 .2 Background shear ................. 123

4.4 Discussion . . . . . . . . . . . . . . . 124

5 Final Conclusion $\quad 128$

A Atlantic Stability Distribution and South Atlantic $\theta-S$ variations near the Mid-Atlantic Ridge

B Mode Interactions

140 


\section{Chapter 1}

\section{Geothermal Forcing}

\subsection{Introduction}

The ocean is forced at its upper boundary by wind stress and by heat and salt fluxes. It is also forced at its lower boundary, because the interior of the earth is hot, and this gives rise to a heat flux at the ocean bottom. The total input to the ocean by this geothermal source according to theoretical models is about $3 \times 10^{13} \mathrm{~W}$ (Sclater, Jaupart, and Galson, 1980). In comparison, the magnitude of the net heat flux over the northern North Atlantic Ocean, for example, is about $1 \times 10^{15} \mathrm{~W}$ (Gill, 1982). Recently, however, it has been demonstrated that the main contact between the surface of the earth and the hot interior occurs at the mid-ocean ridge crest, where new crust is formed as upwelling magma solidifies (see e.g., Cox, 1973). Away from the crest, the age of the ocean bottom increases, the seafloor subsides, and the heat flux into the ocean decreases. The variation of bottom depth and of conductive heat flux away from the crest with age is accurately defined by measurements and theoretical models have been developed which account for it (Sclater et al., 1980). Measurements of the conductive heat flux in the rift valleys at the crest, however, show a great deal of scatter and the net input of heat there by this mechanism is not accurately defined (Morgan, 1982). In addition, there is a discrepancy near the crest between the average conductive heat flux measurements and the predicted total heat flux. Both the scatter and the discrepancy are explained as a result of a convective or hydrothermal component of heat transfer, which amounts to about $1 \times 10^{13} \mathrm{~W}$ (Sclater et al., 1980). If this component alone were spread out over an active part of the ridge system, thought to be confined to a width less than $200 \mathrm{~km}$ centered at the crest and to be of total extent about $50,000 \mathrm{~km}$, then the resulting average heat flux is $1 \mathrm{~W} / \mathrm{m}^{2}$. Sclater et al. (1980) give a heat flux value of about $0.89 \mathrm{~W} / \mathrm{m}^{2}$ for seafloor of age less than $1 \mathrm{Ma}$ (units of $10^{6}$ years before present), which essentially corresponds to the region of a rift valley.

These values are only roughly one percent of an average surface value in the Gulf Stream region, for example, where there is a net heat loss (Gill, 1982), but the 
ability of the geothermal source to drive vertical motion is enhanced because the stratification in deep water is usually more than an order of magnitude weaker. In terms of vertical motion, the strength of forcing at the crest seems significant, and this motivates an investigation of its effect. It should be emphasized that since the area of the crest is small compared to the rest of the ocean, the geothermal source cannot compete with atmospheric forcing at high latitudes in the creation of the general circulation of deep water. That does not prevent geothermal heating from being important in limited regions.

The combination of buoyancy forcing with the injection of passive tracers like helium and manganese (see below) measurable at a large scale seems to be ideal for making inferences about flow (Figure 1.1). In addition, the geothermal sources are especially useful because they occur for the most part at mid-depth in the water column. At these depths relatively little is known about the general circulation away from boundaries. Furthermore, the dynamical processes associated with such sources have not received much attention in the past. Thus from several points of view it seems worthwhile to study this phenomenon. While much work has been done in the fields of geology, geophysics, and chemistry to study geothermal sources, almost no work has been done previously to address the physical oceanographic aspects of this problem. The object of this thesis is to analyze the effect of geothermal sources on the circulation, temperature, and salinity fields of the deep ocean. In particular the procedure will be to show how these fields are affected near such a source, to demonstrate their connection to large-scale structure in the basic stratification, to idealize the physics in an analytical model and to check the results in a numerical simulation.

\subsection{Origin of Geothermal Sources at Ocean Ridge Crests}

The interior of the earth is thought to be maintained at high temperature by radioactive decay. The hot interior gives rise to a heat flux at the solid earth's surface of typical magnitude $0.05 \mathrm{~W} / \mathrm{m}^{2}$ at the ocean floor (Langseth and Von Herzen, 1971). Their compilation of conductive heat flux measurements also showed that at mid-ocean ridges the average is somewhat higher, with maxima sometimes an order of magnitude larger, though the data are highly scattered there. Although the missing convective component of heat flux would raise the the average value, it was not clear that the profiles of heat flux from the abyssal plains to the ridge crests were very supportive of new theoretical models of plate spreading. More recent compilations using only data from well sedimented areas where the conductive component is a good measure of the total heat flux show less scatter and better agreement with the theoretical models (Sclater et al., 1980). 
Influence of Geothermal Sources
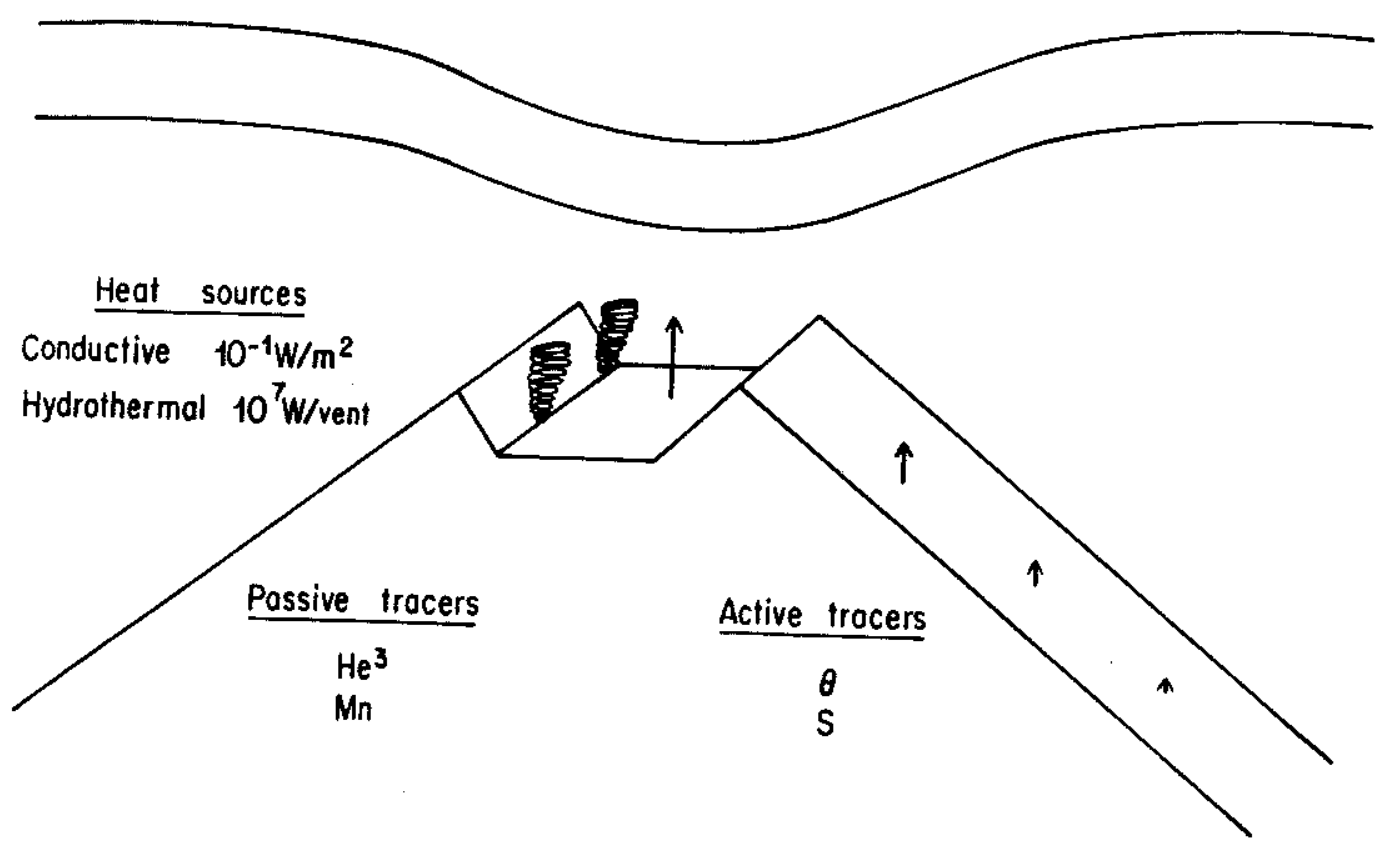

Figure 1.1: Schematic of a segment of a mid-ocean ridge, showing conductive (arrows) and convective (hydrothermal) contributions to heat flux concentrated near the crest. These sources affect the potential temperature $(\theta)$ and salinity $(S)$ fields. Above the ridge segment a large-scale isopycnal deformation is forced, which is not necessarily symmetric. Tracer sources injected at the crest include helium (He) and
manganese $(\mathrm{Mn})$. 
Early measurements of this nonuniformity of heat flux were one of the key ingredients in the development of the plate tectonic model for the evolution of the earth's surface (Cox, 1973). The other key ingredients which are explained in the model are the existence of mid-ocean ridges with a rift valley at their crest, the lack of sediments covering the crest, the concentration of earthquakes along these ridges, and the presence of strong magnetic anomalies forming alternating positive and negative bands off-axis. All of these phenomena are explained by formation of new crust at the mid-ocean ridges. The idea is that convection cells in the mantle divide the crust into plates. At the ridges the plates are spreading, and molten rock fills the gap. Elsewhere the plates slide against one another or subduct one under another, eventually melting and destroying old crust. For this reason the oceanic crust is younger than the continents, which float in the plates and are generally older than $250 \mathrm{Ma}$. Each year, on average, about $30 \mathrm{~km}^{3}$ of new crust is estimated to form. This rock must cool from temperatures near $1000^{\circ} \mathrm{C}$ to ambient seawater temperatures by releasing heat to the ocean. Some of the heat is transferred by conduction, but the ridge is porous, so heat can be removed by convection as well.

The ridges themselves exist because the material which makes them up is warm and buoyant, and therefore floats higher on the underlying layer. The magnetic bands reflect the orientation of the earth's magnetic field when the new crust was formed. This and other dating techniques put the spreading rates between about 1 $\mathrm{cm} / \mathrm{yr}$ and $10 \mathrm{~cm} / \mathrm{yr}$, varying from region to region. The overall trend in spreading rates following the ridge system is from about $1 \mathrm{~cm} / \mathrm{yr}$ in the northern North Atlantic, to $2 \mathrm{~cm} / \mathrm{yr}$ in the South Atlantic, to $3 \mathrm{~cm} / \mathrm{yr}$ in the Indian Ocean, increasing to about $6 \mathrm{~cm} / \mathrm{yr}$ in the equatorial Pacific (Cox, 1973).

Other properties besides heat are transferred at the ridge crest. Seawater, which has many ingredients, percolates into the ridge to depths about $1 \mathrm{~km}$ below the sea floor and comes into contact with hot rock at temperatures greater than $300^{\circ} \mathrm{C}$. Many reactions take place before the water exits, in part because the heated water becomes quite acidic, and thus capable of dissolving more material (Edmond et al., 1982). As a result of these reactions the composition of vent water is very different from that of ambient seawater. Some of the more important constituents of vent water for tracing its dispersal are helium-3, manganese, methane, silica and particulates. The particulates are composed of various minerals which precipitate when the vent water mixes with ambient seawater. Both helium-3 and manganese have been observed thousands of kilometers from their sources along ridge crests, and so are important tracers at the large-scale (Lupton and Craig, 1981; Klinkhammer and Hudson, 1986). Methane, silica, and particulate concentration have been useful for mapping the dispersal closer to the source, at scales less than several hundred kilometers (see e.g. Campbell and Gieskes, 1984). These compositional 
effects can have dynamical consequences in addition to providing tracers. The peculiar compositon of the vent water implies that calculating density by the standard method of measuring conductivity and temperature, and using a reference compositon of seawater to convert conductivity to salinity may not be valid. The problem is certainly insignificant at the large-scale because of dilution, but perhaps not at the small-scale, near the source, and needs to be addressed in the future. It is ignored here in the absence of data appropriate to study the effect.

The final point regarding the composition has to do with salinity. At the high temperatures encountered within the crust, seawater can separate into a "salty" phase, with high concentrations of dissolved salts and a relatively fresh phase (Stefánsson and Björnsson, 1982). Observed salt concentrations in vent water ranging from $15 \%$ to $50 \%$ have been used as evidence for this separation. Salinity variations of this magnitude make salt of similar importance to the total buoyancy flux from a source as temperature, and thus modify the spreading of vent water and the nature of its influence.

\subsection{Regional Distribution and Strength}

The dominant pattern in the distribution of heat flux at the ocean bottom is the concentration of high values along ridge crests. A convenient formula based on measurements and models for the total average heat flux is $0.5 / t^{1 / 2} \mathrm{~W} / \mathrm{m}^{2}$, where $t$ is the age of the bottom in $\mathrm{Ma}$ (Morgan, 1982; age varies from $0 \mathrm{Ma}$ at the crest to about $250 \mathrm{Ma}$ near trenches). This formula diverges at $t=0$, but the theoretical mean heat flow for age less than 1 Ma given by Sclater et al. (1980) is $0.89 \mathrm{~W} / \mathrm{m}^{2}$.

The distribution of conductive and hydrothermal heat flux along the crest is somewhat chaotic, but larger-scale patterns are beginning to appear (Crane et al., 1985). Variations in the heat flux are caused by variations in the properties of the crust and by mechanical changes such as fractures. Also, the nature of the underlying magmatic convection process may impress certain scales on the alongcrest distribution of heat flux (Crane et al., 1985). This latter process is suggested to be related to the occurrence of hot spots separated by about $100 \mathrm{~km}$ along a portion of the Juan de Fuca Ridge in the Pacific near $47^{\circ} \mathrm{N}, 130^{\circ} \mathrm{W}$. Associated with explored hot spots are vent systems consisting of both high-temperature (approx. $300^{\circ} \mathrm{C}$ ) individual sources and low-temperature (approx. $25^{\circ} \mathrm{C}$ ) diffuse or spread-

out sources. Both types of sources are expected to transport similar amounts of heat, but it has not proven possible to measure the heat flux from diffuse sources with much confidence thus far (Converse et al., 1984). Individual high-temperature sources, on the other hand, typically have a chimmey-like structure and direct measurements can be made. 
The results of direct measurements on individual vents give a typical heat output of $1 \mathrm{MW}$ to $10 \mathrm{MW}$ (Converse et al., 1984). These measurements were made by inserting a flowmeter and thermometer directly into the chimney. The total number of sources at a vent system can be many hundreds; Converse et al. (1984) estimate a total heat output by three vent systems at $21^{\circ} \mathrm{N}$ on the East Pacific Rise of $2.2 \times 10^{8} \mathrm{~W}$. This figure includes an estimated contribution by low-temperature diffuse sources of about $35 \%$. At least one other unmeasured hightemperature system exists at this site, but it is not known how many sources there are altogether in the region. These three vent systems occupy an area roughly $1000 \mathrm{~m}$ by $50 \mathrm{~m}$. Within this limited area, the average heat flux is then $4400 \mathrm{~W} / \mathrm{m}^{2}$. Averaged over an entire ridge segment $10 \mathrm{~km}$ by $10 \mathrm{~km}$ the flux is about $2 \mathrm{~W} / \mathrm{m}^{2}$. The conclusion of Converse et al. (1984) for this site was that such a strong heat flux cannot be maintained in steady state by the spreading process. They were only able to constrain the lifetime of this vent system to be between $100 \mathrm{yrs}$. and 40,000 yrs. Such a constraint is useful for geological purposes but will be considered here to mean that the source is steady. In other areas of the ridge crest the hydrothermal component may be absent, and in addition the conductive component may be weak.

An estimate for the average hydrothermal component of heat flux can be made by adding up individual and diffuse sources. This estimate will use numbers from measurements at the East Pacific Rise; it is not known how representative these numbers are for other ridges. If there is one large vent system in the rift valley every $100 \mathrm{~km}$ at $2.2 \times 10^{8} \mathrm{~W}$ plus one smaller group of vents every $2 \mathrm{~km}$ (Van Doveret al., 1987) at, say, $1 \times 10^{7} \mathrm{~W} /$ group, then the average flux over the rift valley of width $10 \mathrm{~km}$ is about $0.75 \mathrm{~W} / \mathrm{m}^{2}$. This rough estimate is similar to the $0.89 \mathrm{~W} / \mathrm{m}^{2}$ value from the plate model for a seafloor age less than $1 \mathrm{Ma}$ (the model value is essentially independent of spreading rate).

Heating results from the convergence of geothermal heat flux near the bottom. One way to estimate the penetration scale of the resulting convection and the effect on the density field is to consider the formation of a mixed layer near the bottom. The initial temperature and salinity profiles are taken to be linear. Conserving heat in a layer of thickness $h$ means that the temperature of the layer will change according to

where

$$
\frac{\partial}{\partial t}\left(h \rho C_{p} \theta\right)=F+\frac{\partial}{\partial t}\left(h \rho C_{p}<\bar{\theta}>\right)
$$

$$
<\bar{\theta}>=\frac{1}{h} \int_{0}^{h} \bar{\theta} d z .
$$

Here $\theta$ is the potential temperature of the layer, $\bar{\theta}$ is the background profile, $t$ is time, $F$ is the heat flux, and $\rho C_{p}=4.05 \times 10^{6} \mathrm{~J} / \mathrm{m}^{3}{ }^{\circ} \mathrm{C}$ is the specific heat (thermodynamic parameters are from Gill (1982), for seawater at $4000 \mathrm{db}, 2^{\circ} \mathrm{C}$, and $35 \%$ ). The first 
term on the right is the geothermal source supplying heat through the bottom of the layer and the second term is the heat mixed down with water initially above the layer. For constant heating the layer temperature is

$$
\theta=T_{0}+\frac{h}{2} \bar{\theta}_{z}+\frac{F t}{h \rho C_{p}} .
$$

Conserving salt means that the vertical integral of the salinity profile is constant, since it is assumed that there is no source of salt. For an initially linear background profile $\bar{S}=S_{0}+\bar{S}_{z} z$ the salinity of the mixed layer is just the average of the bottom value and the value at height $h$, or

$$
S=S_{0}+\frac{h}{2} \bar{S}_{z}
$$

One more condition is necessary to solve for $h$ which is that at the top of the layer the density difference goes to zero or else the layer would continue to mix upwards. To apply this condition an equation of state is needed:

$$
\rho=\rho_{0}+\alpha\left(\theta-T_{0}\right)+\beta\left(S-S_{0}\right),
$$

where $\alpha=-1.71 \times 10^{-4 \circ} \mathrm{C}^{-1}$ is the thermal expansion coefficient, and $\beta=7.71 \times 10^{-4}$ is the saline contraction coefficient. Then the third condition becomes $\alpha(\theta-\bar{\theta}(h))+$ $\beta(S-\bar{S}(h))=0$. Substituting the background profiles into this condition gives

$$
\alpha\left(\theta-T_{0}-\bar{\theta}_{z} h\right)+\beta\left(S-S_{0}-\bar{S}_{z} h\right)=0 .
$$

Solving for $h$,

$$
h=\left[\frac{2 F t / \rho C_{p}}{\bar{\theta}_{z}+\frac{\beta}{\alpha} \bar{S}_{z}}\right]^{1 / 2}
$$

Using background gradients from the Mid-Atlantic Ridge (chapter 2) of $\bar{\theta}_{z}=4 \times 10^{-4}{ }^{\circ} \mathrm{C} / \mathrm{m}$ and $\bar{S}_{z}=4 \times 10^{-5 \%} \% / \mathrm{m}$, and a flux of $1 \mathrm{~W} / \mathrm{m}^{2}$, the thickness can be written approximately as $h=266 t^{1 / 2}$ where $h$ is in meters and $t$ is in years. Note that the unstable salinity gradient increases the thickness of the layer. This formula gives a thickness of $100 \mathrm{~m}$ in about 2 months. To penetrate $500 \mathrm{~m}$ requires about 3.5 yrs. The mixed layer thickness is a useful measure of the strength of the heating, but it probably does not represent the penetration height well in regions where the heat flux is nonuniform. If the heating is somehow confined to a limited area, for example as it is at hydrothermal sites, the resulting buoyant plume can quickly penetrate hundreds of meters (Chapter 2).

To estimate the significance of the heating on deep flow, two comparisons have to be made. First, the flow speed $\delta V$ forced by heating must be compared to a 
background flow speed $V$, assumed to be independent of the heating, and then both speeds can be compared to direct and indirect measurements of flow near ridges. Vertical mixing above a heat source on the ridge crest creates horizontal density gradients. The circulation must adjust to the new density distribution toward a steady state with some geostrophically balanced flow around the heat source. An estimate of this flow is

$$
\delta V=\frac{g}{\rho f X} \frac{1}{2} \frac{\partial \bar{\rho}}{\partial z}(h)^{2}
$$

where $\delta V$ is the flow speed along the ridge, $g$ is gravity, $f$ is the Coriolis parameter, $X=100 \mathrm{~km}$ is the length scale across the ridge, $\frac{\partial \bar{\rho}}{\partial z}=O\left(10^{-9}\right) \mathrm{cm}^{-1}$ is the mean vertical potential density gradient, and $h$ is the mixing height. The scale $\frac{1}{2} \frac{\partial \bar{\rho}}{\partial z} h$ is used for the horizontal density change. The strength of the flow $\delta V$ is thus set by the mixing height $h$, which is related to the residence time of water at the crest by 1.6. For example, a background flow of $V=0.5 \mathrm{~cm} / \mathrm{s}$ on a $500 \mathrm{~km}$ path along the crest gives a residence time of about 1000 days. Over this time period the water mixes to an average thickness of roughly $220 \mathrm{~m}$, and with this value, the velocity $\delta V=0.2 \mathrm{~cm} / \mathrm{s}$. This value is comparable to $V$ but not strong enough to reverse the background flow. This equation may be written in terms of the heat flux by substituting 1.6 into 1.7 , giving

$$
\delta V=\frac{g \alpha F t}{f X \rho C_{p}} .
$$

To have a significant effect, the forced flow ought to be the same size as the background flow. Setting $\delta V=V$ for a fixed $500 \mathrm{~km}$ path length determines $\delta V=V=0.3 \mathrm{~cm} / \mathrm{s}$ from the approximate formula

$$
\delta V=\left(\frac{g \alpha F t}{f \rho C_{p}} \frac{\sqrt{X^{2}+Y^{2}}}{X}\right)^{1 / 2}
$$

where $Y$ is the length scale along the ridge. A minimal path length has $X \sim Y$, and this choice gives the conservative value $\delta V=V=0.1 \mathrm{~cm} / \mathrm{s}$.

Several estimates of flow speed near the Mid-Atlantic Ridge and East Pacific Rise have been made. Roemmich and Wunsch (1985) show geostrophic velocity at $24^{\circ} \mathrm{N}$ in the Atlantic determined by inverse methods. A shallow initial reference choice results in a flow reversal from west to east across the ridge, with southward flow of about $0.5 \mathrm{~cm} / \mathrm{s}$ above the western flank and northward flow of about $0.2 \mathrm{~cm} / \mathrm{s}$ above the eastern flank (their Figure 17a). Also, year-long current meter measurements at $4000 \mathrm{~m}$ from Polymode cluster $\mathrm{C}$ at $16^{\circ} \mathrm{N}, 54^{\circ} \mathrm{W}$ above the western flank of the Mid-Atlantic Ridge show mean velocities of about $0.4 \mathrm{~cm} / \mathrm{s}$ to $1 \mathrm{~cm} / \mathrm{s}$ directed to the north and west (Kamenkovich, Monin, and Voorhis, 1986). A similar velocity of about $0.4 \mathrm{~cm} / \mathrm{s}$ near $3000 \mathrm{~m}$ depth along the East Pacific Rise near $15^{\circ} \mathrm{S}$ is implied by 
the map of adjusted steric height by Reid (1986). Reid (1982) estimates a westward geostrophic speed at $2500 \mathrm{~m}$ depth relative to $3500 \mathrm{~m}$ depth of $0.24 \mathrm{~cm} / \mathrm{s}$ above the East Pacific Rise near $15^{\circ} \mathrm{S}$. All of these speeds are in the range $0.1 \mathrm{~cm} / \mathrm{s}$ to $1 \mathrm{~cm} / \mathrm{s}$. At present one cannot tell whether these measurements are of a background flow or of a flow forced by heating, but even if they are for a background flow the above scale analysis suggests that the residence time on the ridge may be long enough for a significant heating effect.

Joyce, Warren and Talley (1986) showed that an average heat flux of $0.05 \mathrm{~W} / \mathrm{m}^{2}$ on the abyssal plains well away from mid-ocean ridges has a negligible dynamic effect on the vertically integrated deep flow. Thus they could use the temperature anomaly generated by geothermal heating as a passive tracer. It was noted that vertical shear induced by the heating was small, but perhaps not insignificant (a turning of a $0.7 \mathrm{~mm} / \mathrm{s}$ flow through an angle less than $30^{\circ}$ in the lower kilometer of the water column). An average heat flux ten times larger near ridge crests does seem to be strong enough to modify deep flow of this magnitude.

An analogy can be made between the geothermal heating of the ocean at midocean ridges and the heat flux from the ocean to the atmosphere near an oceanic western boundary. The lower part of the atmosphere is heated and humidified as it flows over the warm ocean currents there. The analogy is quantitative in the sense that the net source of heat to the atmosphere from warm water near the western boundaries is about two orders of magnitude smaller than the solar heat flux to the atmosphere over an area the size of an ocean (Gill, 1982). Thus the ratios of the two sources in each system is the same. In the atmosphere the zonal flow is strong but can be modified by the ocean-atmosphere heat flux (Gill, 1982). In most of the ocean continental boundaries prevent the development of similar zonal flow, so that the flow generated by the geothermal heating may be less likely to be overwhelmed by flow generated by other mechanisms.

\subsection{Related Studies and This Work}

The dynamical effect of geothermal sources in deep water has not received much attention, yet they seem to be capable of modifying the deep flow. Furthermore the connection between the smaller-scale and larger-scale motion, tracers, and temperature salinity anomalies has not been clear. Observations of tracers such as helium-3 and manganese in the South Pacific have revealed large-scale plumes indicating both westward and eastward mid-depth flow (Lupton and Craig, 1981; Klinkhammer and Hudson, 1986). The relationship between these tracers and the density field has been shown in maps (Edmond et al., 1982; Reid, 1986), but no explanation has been given to suggest the reason for agreement or for disagreement 
between them in different regions.

A temperature-salinity anomaly similar in scale to the helium-3 plume has been observed in the South Pacific by Reid (1982). The source of the anomaly is geothermal heating at the East Pacific Rise. Larger-amplitude but smaller-scale anomalies have been observed at many locations in the North and South Pacific above the East Pacific Rise (Crane et al., 1985). Such anomalies however, have not previously been reported to occur near the Mid-Atlantic Ridge. Saunders and Francis (1985) explored a portion of the Mid-Atlantic Ridge between $43^{\circ} \mathrm{N}$ and $47^{\circ} \mathrm{N}$ by making vertical temperature profiles, and found warm, well mixed bottom layers in isolated segments of the ridge. These layers were thought to be influenced by the conductive heat flux, but since salinity was not measured anomalous temperature-salinity values could not be reported. Farther south, between $26^{\circ} \mathrm{N}$ and $11^{\circ} \mathrm{N}$, Klinkhammer et al. (1985) showed evidence for hydrothermal activity, based on manganese measurements. Again, no temperature-salinity anomalies were presented, nor have any chemical signals of hydrothermal sources been reported outside the rift valley in the Atlantic. Broecker and Takahashi (1980) illustrate a temperature-salinity anomaly in several profiles taken about $1000 \mathrm{~km}$ west of the Mid-Atlantic Ridge from $5^{\circ} \mathrm{S}$ to $15^{\circ} \mathrm{S}$. They suggest that geothermal heating at the ridge crest in the same latitude range is responsible for the anomaly. Unfortunately stations closer to the ridge were not available to them to show the connection.

One of the objectives of this work is to analyse the effect of heat and salt sources on the temperature and salinity fields of deep water in the Atlantic Ocean. Special emphasis is given to the region of the Mid-Atlantic Ridge near $26^{\circ} \mathrm{N}$ because new measurements from this region have become available. These measurements constitute the first observations of the physical characteristics of a geothermally active portion of the Mid-Atlantic Ridge, and it is the only such location in the Atlantic where good CTD coverage exists. The other objective is to study the circulation forced by heating. In part, this involves the relation between the passive tracers and active tracers (such as temperature) injected at the source. In the case of the South Pacific, the combination of local heating, driving westward flow, and a general upwelling of deep water, driving eastward flow, is necessary to explain the distribution of properties (Chapter 3 ).

It will be convenient to divide the response of the ocean to heating into three regimes, the small-scale, intermediate-scale, and large-scale response. The horizontal length scales which characterise these regimes are $O(100 \mathrm{~m}), O(10 \mathrm{~km})$, and $\mathrm{O}(1000 \mathrm{~km})$ respectively. The dynamical (i.e., time scale) basis for this division will be made clear when models of the response in each of the three regimes are presented in subsequent chapters.

Motion in the small-scale regime is generated by individual sources such as hot 
springs or locations of high conductive heat flux. Above these sources heated water rises in a turbulent plume, entrains, and finally spreads out at some equilibrium level where the density matches that of the surrounding water. The dynamics of this process was presented by Morton, Taylor, and Turner (1956), based on the assumption of a linear proportionality between the average vertical motion and the entrainment. The same assumption is used in this study but the plume model is extended to separate the effects of temperature and salinity on the vertical structure of the plume (Chapter 2). With this extension the temperature-salinity characteristics of the plume can be predicted, and the effect of different background salinity and temperature fields representing the Atlantic and Pacific can be explored. The results presented here describe the prediction and discovery of an anomalously cold, fresh plume in the rift valley of the Mid-Atlantic Ridge, maintained by hot springs on the bottom.

Motion at the intermediate scale is generated by the entrainment and spreading process associated with a turbulent plume, and by the broader upwelling forced by less localized heating elsewhere along the ridge crest. Motion at this scale is closely related to atmospheric convection systems such as hurricanes, and has been termed by Gill (1982) a forced baroclinic vortex. He describes solutions in which the forcing is a specified internal heating representing the latent heat release inside an atmospheric system. In this work an example will be presented in which the forcing is calculated from the small-scale turbulent plume model, and therefore is consistent with the physics of the plume. The example illustrates the displacement of isopycnals which in turn is used as the forcing in a large-scale model. The dominant isopycnal displacement is not a direct result of the heating but of the horizontal flow, generated in response to upwelling at the source.

Motion at the large scale is generated by the average heat flux over long segments of the ridge. Stommel (1982) argued that geothermal heat sources along the East Pacific Rise in the South Pacific were strong enough to force deep flow. Although he did not present solutions to the equations he did sketch the expected pattern of flow resulting from a mass source (upwelling) at a ridge crest. His model was linearized about a resting background state, so there was no competing flow forced by some other mechanism. One of the goals of this study is to examine the large-scale influence of a geothermal heat source in the presence of a non-uniform background flow, and to include nonlinear effects. The kind of nonlinearity which can be important is not inertial, because the flow is weak, but rather the lateral advection of heat. The general condition that this effect is significant is that the particle speed be comparable to the long wave phase speed (Chapter 3 ). This condition appears to be satisfied in some regions for a speed of order one centimeter per second. Various non-uniformities in the background field, together with nonlineari- 
ties, are considered in an analytical model (Chapter 3) and a numerical simulation (Chapter 4).

\subsection{Conclusion}

In summary, relatively strong geothermal heat flux occurs at mid-ocean ridges, where earth's plates are spreading apart owing to convection in the mantle. An estimate of the average heat flux near the crest is $1 \mathrm{~W} / \mathrm{m}^{2}$, and it seems plausible that this forcing can generate deep flow. In addition, geothermal sources introduce a combination of passive and active tracers at mid-depth, where not much is known about the general circulation. This work consists of a descriptive and dynamical investigation of the influence of these sources in the deep ocean at several scales, from the immediate vicinity of a source to the basin as a whole. 


\title{
Chapter 2
}

\section{The Evidence for the Influence of Geothermal Heating on the Temperature and Salinity Fields in the Tropical Atlantic.}

\begin{abstract}
Temperature and salinity measurements from CTD stations in the rift valley of the Mid-Atlantic Ridge at $26^{\circ} \mathrm{N}$ and from several zonal sections across the Atlantic are examined for evidence of geothermal heating. Data from the rift valley show the presence of an anomalously cold, fresh plume originating at a hydrothermal site consisting of many low-temperature and high-temperature sources. A turbulent entrainment model of the buoyant plume predicts the observed penetration height and $\theta-S$ characteristics of the plume, and for comparison, characteristics of a plume from the Pacific are also shown. One consequence of the entrainment occuring in the plume is the generation of a circulation around the source which may be strong enough to trap fluid near the source in the presence of background flow. A model of this flow is presented. The large-scale distribution of salinity is examined on two isopycnal surfaces corresponding to depths of roughly $3500 \mathrm{~m}$ and $4200 \mathrm{~m}$ in the western trough of the North Atlantic. The distribution is dominantly that expected from the movement of deep and bottom water in boundary currents. A weak salinity minimum in a limited region around the Mid-Atlantic Ridge near $15^{\circ} \mathrm{N}$ is suggestive of a local heating effect.
\end{abstract}

\section{$2.1 \quad$ Introduction}

The purpose of this Chapter is to present a study of the physical oceanography near newly discovered hot springs in the rift valley of the Mid-Atlantic Ridge, and to use these specific results to infer the effect of geothermal heating as a whole (hydrothermal plus conductive components) on deep water near the level of the crest. The existence of a large-scale signal of geothermal sources is well known in the Pacific (Edmond et al., 1982). However, no large-scale signal has been observed 
and identified previously in the Atlantic.

There are two other reasons that this observational work focuses on the Atlantic: 1) the availability of a unique CTD data set surrounding the first active vents to be discovered on the Mid-Atlantic Ridge; 2) the existence of several modern CTD sections in which weak signals may be observable.

\subsection{Data, Methods}

Data for this study come from several sources. CTD data at the Trans-Atlantic Geotraverse (TAG) site at $26^{\circ} \mathrm{N}$ on the Mid-Atlantic Ridge and at several locations between this site and $10^{\circ} \mathrm{N}$ were provided by P. Rona at NOAA (Atlantic Oceanography and Meteorology Laboratory, Miami, Florida; data collected in August, 1984, and August, 1985; Rona et al., 1986; Figure 2.1).

The temperature measurements are believed to be accurate to $\pm .005^{\circ} \mathrm{C}$. At the time of the cruise (1984), the sensor was calibrated once per year. The precision is $\pm .001^{\circ} \mathrm{C}$. Bottle sampling was inadequate to calibrate CTD salinity. Salinity profiles were therefore calibrated by comparing the near bottom $\theta-S$ relation to that at station 189 of ATLANTIS II cruise 109, the nearest station of known high quality. It was found that a constant offset of $0.013 \%$ added to the TAG data salinity values made the $\theta-S$ relations agree. The vertical structure in the profiles is thought to be reliable, except in a few places where there are obvious lag problems between the temperature and conductivity sensor. Except for these points the precision is $\pm .001 \%$. Only the lower $1000 \mathrm{~m}$ or $2000 \mathrm{~m}$ of the profiles is used, so no attempt was made to adjust upper water values. Pressure measurements are thought to be accurate to $\pm 5 \mathrm{db}$.

These CTD measurements were designed to be exploratory; the discovery of active vents was a surprise. The measurement of salinity near hydrothermal vents anywhere has been problematic because the composition of the water varies. Thus standard-water calibrations of the salinometer, which assume a fixed composition to convert conductivity measurements to salinity values, may not be appropriate. Future measurements must address this problem, perhaps by direct density measurements.

Temperature and salinity data at $24^{\circ} \mathrm{N}$ (from observations made in August, 1981 ) and $36^{\circ} \mathrm{N}$ (June 1981) come from ATLANTIS II cruise 109, (Roemmich and Wunsch, 1985). Unpublished data at $13^{\circ} \mathrm{N}$ (January 1983), $23^{\circ} \mathrm{S}$ (February 1983), and $53^{\circ} \mathrm{W}$ (April 1983) from OCEANUS cruise $133 \operatorname{legs} 2,4$, and 7 , and at $35^{\circ} \mathrm{W}$ (June 1983) from KNORR cruise 104 were provided by M. McCartney. Data at $11^{\circ} \mathrm{S}$ (March 1983) came from OCEANUS cruise 133, leg 5 by B. Warren. Data at $45^{\circ} \mathrm{N}$ (April 1980) came from OCEANUS cruise 78 , and data at $64^{\circ} \mathrm{W}$ (April 1985) 
a

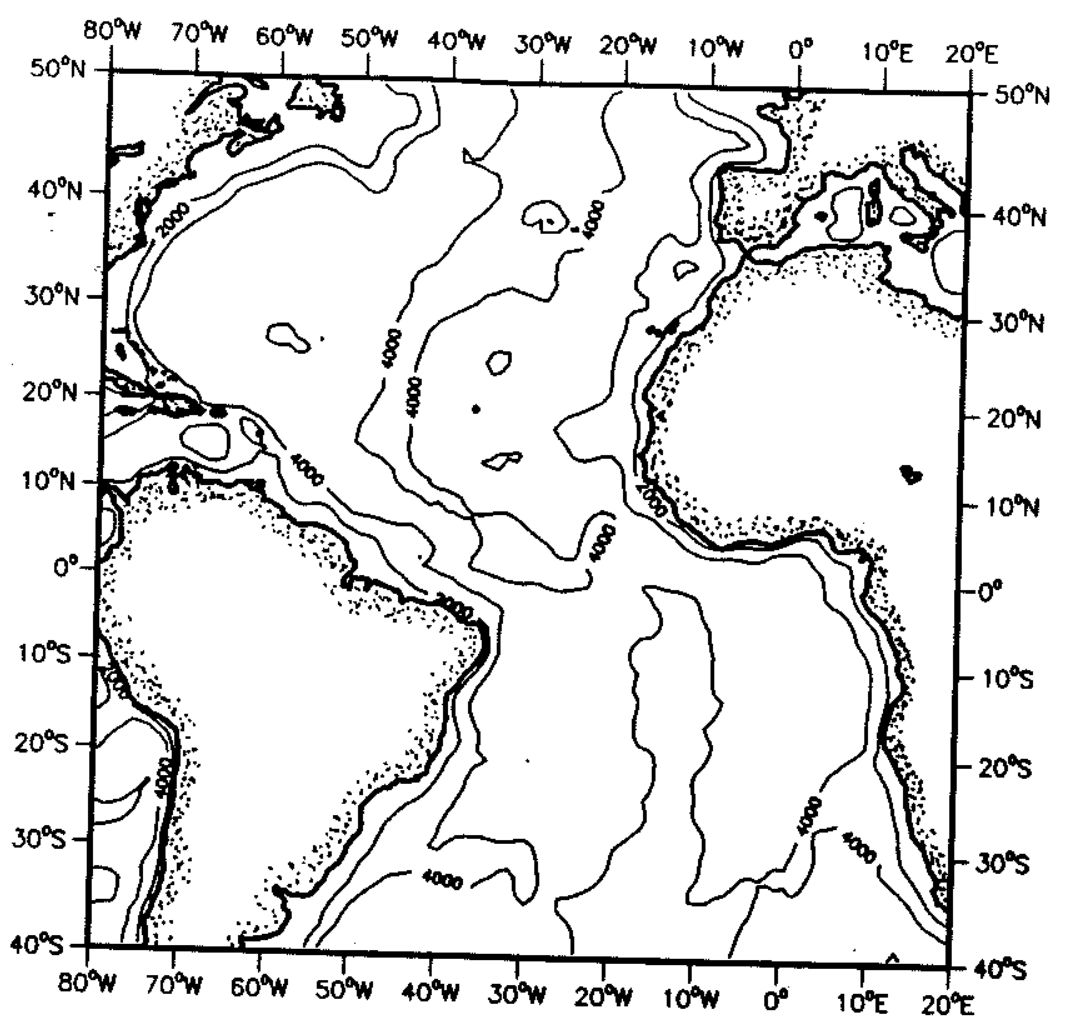

b

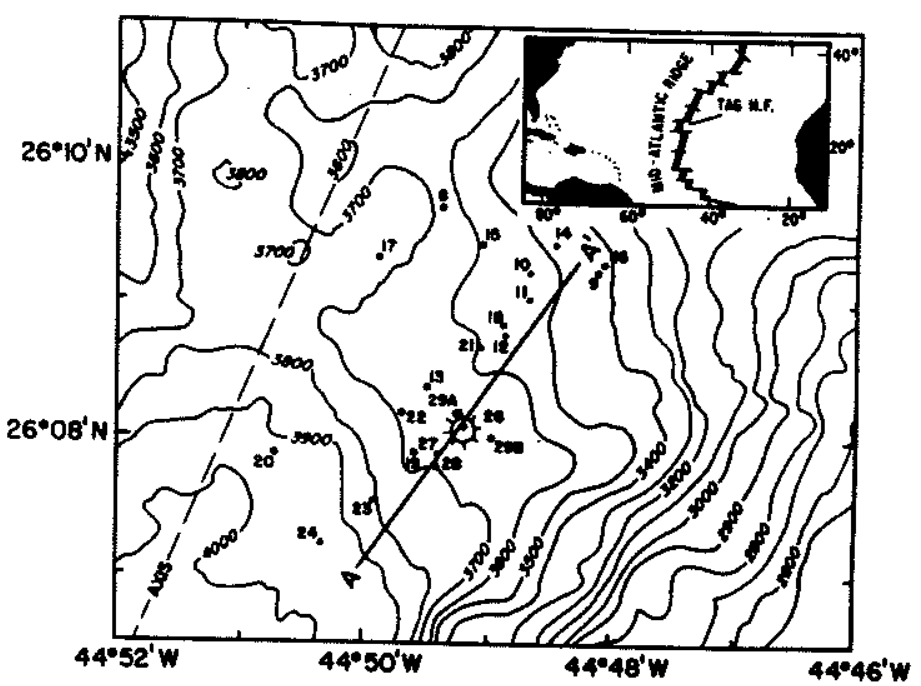

Figure 2.1: a) Atlantic Bathymetry. b) Location of CTD casts at the TAG hydrothermal area. Bottom depth is in meters. The line AA' is the line onto which stations are projected to make a temperature section (Figure 2.6). The hydrothermal mound where vents are concentrated (circle with dashes) and the axis of the valley are shown (TAG site bathymetry from Rona and Speer, in preparation). 
came from ENDEAVOR cruise 129. These data were processed by the WHOI CTD group using their standard practices (Millard, 1982). The expected accuracy of the measurements.is $\pm .002^{\circ} \mathrm{C}, \pm .002 \%, \pm 2 \mathrm{db}$ for temperature, salinity, and pressure.

\begin{tabular}{lllll} 
LOCATION & TIME & SHIP AND CRUISE & BATCH & $\Delta S$ \\
\hline $45^{\circ} \mathrm{N}$ & April 1980 & OCEANUS 78 & & \\
$36^{\circ} \mathrm{N}$ & June 1981 & ATLANTIS II 109 leg 1 & P81 & +.001 \\
$24^{\circ} \mathrm{N}$ & August, 1981 & ATLANTIS II 109 leg 3 & P81 & +.001 \\
$13^{\circ} \mathrm{N}$ & January 1983 & OCEANUS 133 legs 2 & P90 & -.002 \\
$23^{\circ} \mathrm{S}$ & February 1983 & OCEANUS 133 leg 4 & & \\
$11^{\circ} \mathrm{S}$ & March 1983 & OCEANUS 133 leg 5 & P93 & 0 \\
$53^{\circ} \mathrm{W}$ & April 1983 & OCEANUS 133 leg 7 & P93 & 0 \\
$35^{\circ} \mathrm{W}$ & June 1983 & KNORR 104 & P93 & 0 \\
$64^{\circ} \mathrm{W}$ & April 1985 & ENDEAVOR 129 & P93 & 0 \\
TTO & $1981-1983$ & KNORR & P80 & +.004 \\
GEOSECS & $1972-1973$ & KNORR & &
\end{tabular}

Oxygen and nutrient measurements were also made on the WHOI cruises. These data are expected to be accurate to $\pm 0.05 \mathrm{ml} / 1$ for oxygen, and $1 \%$ for the nutrients (only silica is used here).

Other data used here are from the Geochemical Ocean Section Study (GEOSECS) program (July 1972 to April 1973 for Atlantic stations; Bainbridge, 1980), and the Transient Tracers in the Ocean (TTO) program (North Atlantic Study: April 1981 to October 1981; Tropical Atlantic Study: December 1982 to February 1983; Brewer, Sarmiento, and Smethie, 1985). The accuracy of these data is not reported, but is expected to be similar to the WHOI measurements. A few values from the Levitus Atlas (Levitus, 1982) are used where noted.

Salinity values were corrected for differences between standard sea water batches used for calibration according to Table 2 from Mantyla (1986). The corrections $(\Delta S)$ are displayed with batch numbers in the table given here. During the TTO program both standard sea water batch $\mathrm{P} 80$ and batch $\mathrm{P} 90$ were used to calibrate the salinometer measurements, but the reported data are referenced to batch P80 (Mantyla, 1986). A mean offset on a deep isopycnal surface between WHOI data and the TTO data of $+.004 \%$ (with a standard deviation of $.002 \%$ ) was found in places where they overlapped, so this value was added to TTO data used here rather than Mantyla's (1986) correction of $+.003 \%$. These corrections improved the consistency of salinity measurements between the different data sets. A comparison of GEOSECS salinity to TTO salinity in nearby stations revealed a trend from about $.004 \%$ salty to $.004 \%$ fresh, which produced a standard deviation of $.008 \%$ of the difference. Some of this variability may be real, from changes in time, but the better agreement between the other data suggests some calibration error. The GEOSECS salinity was not used here. 
Not all stations in a given cruise were used for making the horizontal maps of properties on constant density surfaces in areas away from the main region of interest near the Mid-Atlantic Ridge. This was justified by a few plots that were made with all the stations, which did not show any new structure. The locations of missing stations are easily visible in the maps as portions of cruise track with unusual or wide spacing.

The method used is first to examine the variations of the basic variables (temperature and salinity), and of several derived quantities. An attempt is made to infer from these variations some process represented in the heat or salt conservation equations. These processes include horizontal and vertical advection and diffusion, and sources of heat and salt. These patterns are assumed to be stationary, and the effect of eddies and other small-scale motion is assumed to be contained in turbulent diffusion coefficients.

\subsection{Near Field Observations}

A vertical profile of potential temperature at the TAG area indicates the presence of hydrothermal activity (Figure 2.2, solid line, cast 26). The depth range is from $2000 \mathrm{~m}$, which is about at the level of the mountain range on either side of the rift valley, to the bottom. The profile is typical of deep profiles above the MidAtlantic Ridge except for the layer of rapid variation in temperature near $2.5^{\circ} \mathrm{C}$ between $3300 \mathrm{~m}$ and $3400 \mathrm{~m}$ depth. In this depth range there are several inversions where cold water overlies warm water. The magnitude of the variation is about $0.05^{\circ} \mathrm{C}$. These variations could only have as their source the underlying hot springs since surrounding casts had only weaker or no variation. Particulate measurements indicate that the CTD went through black smoke at this level, which is made up of mineral percipitates resulting from the mixture of pure vent water with ambient seawater. The hot vent water evidently rises $300 \mathrm{~m}$ to $450 \mathrm{~m}$ above the bottom as a buoyant plume, while mixing with the surrounding seawater.

These temperature variations are not fully compensated by salinity variations to produce a stable profile (Figure 2.3), indicating that active convective overturning is occurring in the part of the plume sampled by the CTD. The plume does appear, though, to have reached its maximum level of upward penetration; no other profiles had significant variations at lower densities. Cast 26 profiled within a few hundred meters of the center of a major plume from the TAG site, passing through its equilibrium level.

For comparison, two stations from a section at $24^{\circ} \mathrm{N}$ are also shown (Figure 2.2, light and dashed lines), one from the crest and one from the eastern trough. The TAG station is warmer than those farther south by up to several tenths of a degree 


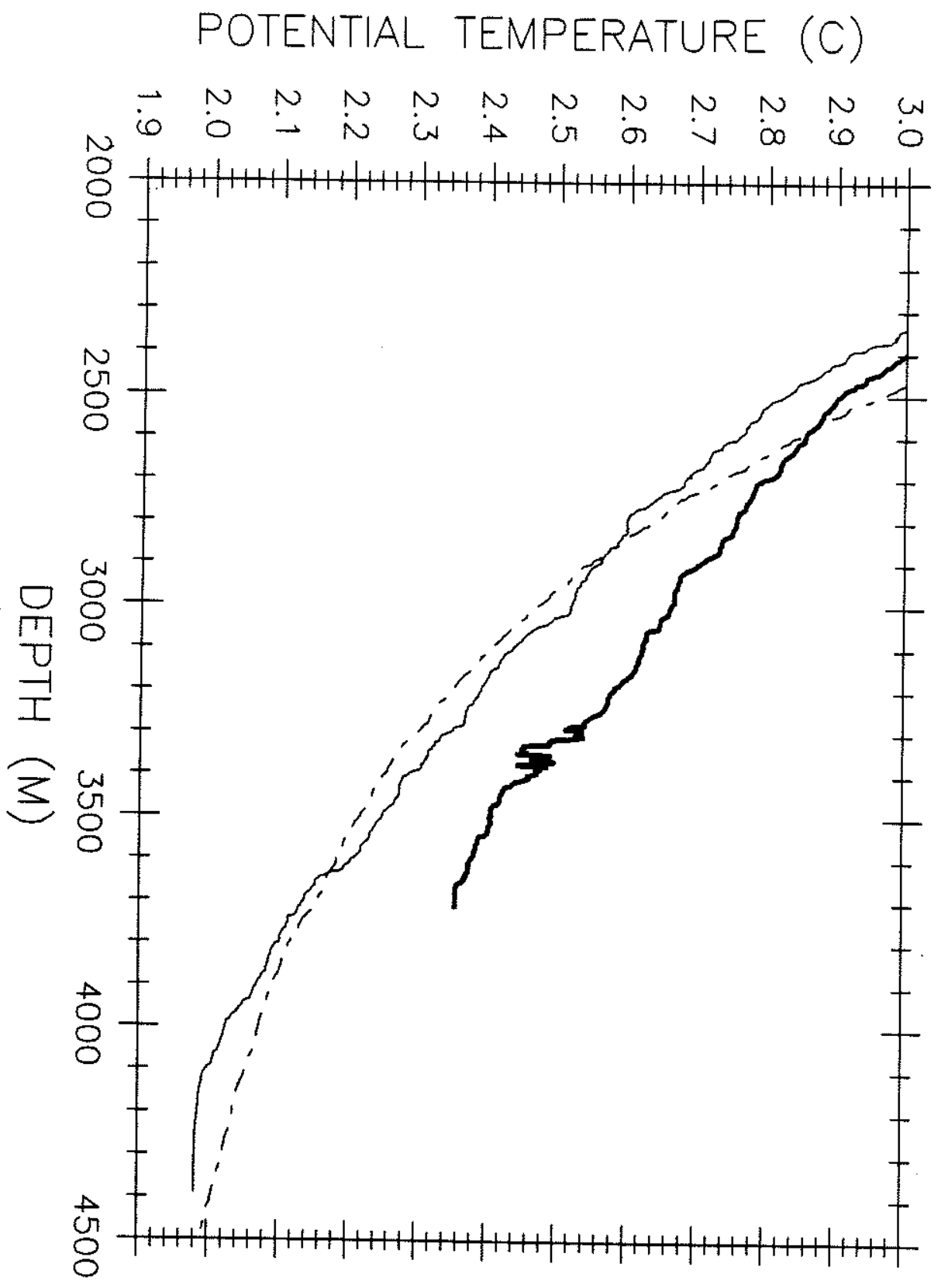

Figure 2.2: Potential temperature-depth curves for TAG cast 26 (heavy solid) and stations 189 (solid, $24^{\circ} 31.05 \mathrm{~N}, 46^{\circ} 9.12 \mathrm{~W}$ ) and 172 (dashed, $24^{\circ} 29.06 \mathrm{~N}$, $\left.31^{\circ} 30.93 \mathrm{~W}\right)$ from a section at $24^{\circ} \mathrm{N}$. 


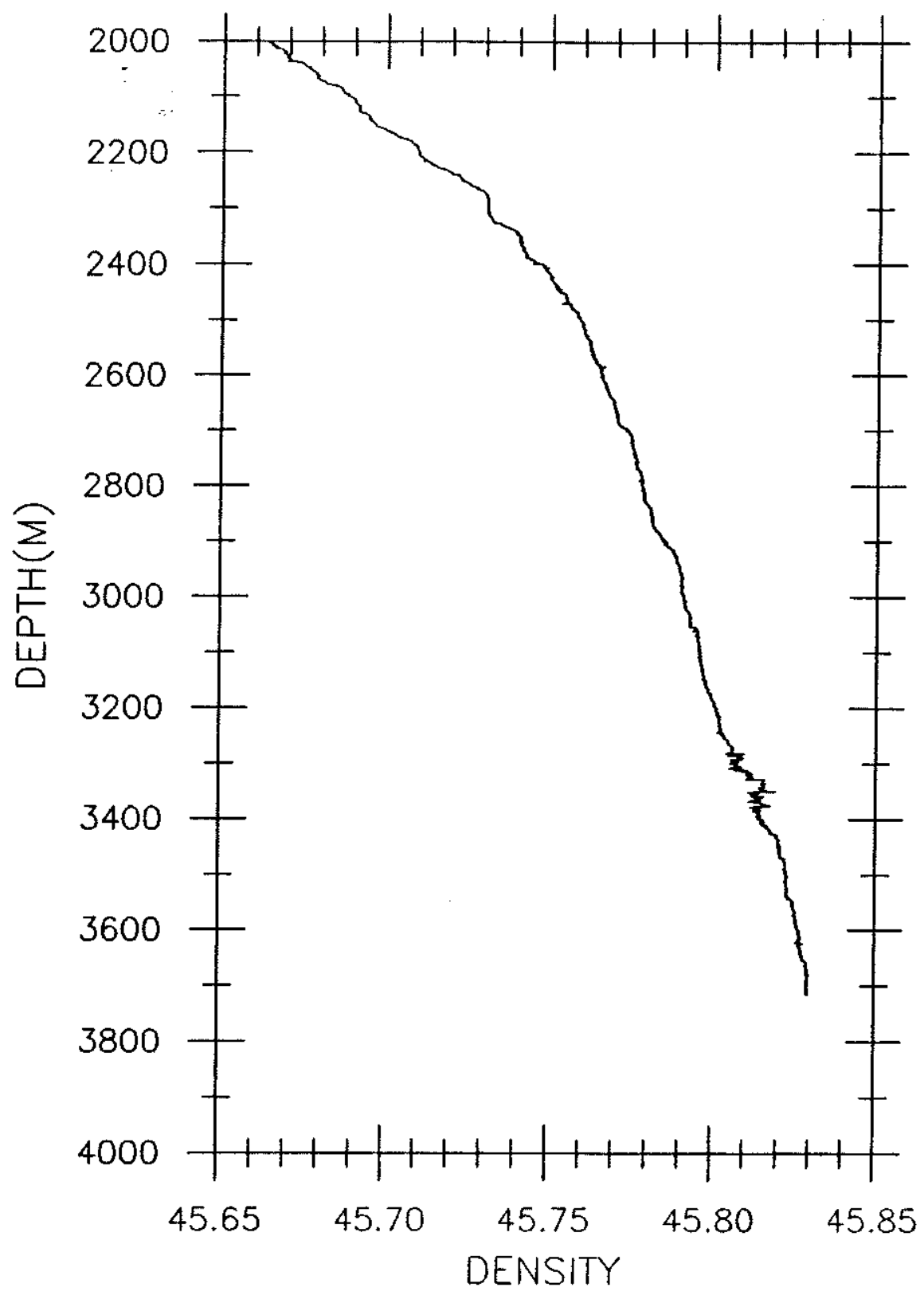

Figure 2.3: Density $\left(\mathrm{g} / \mathrm{cm}^{3}\right)$ referenced to $4000 \mathrm{db}$ versus depth for TAG cast 26 . 
below $2500 \mathrm{~m}$. Thus any large-scale isopycnal spreading of the vent properties might be expected at levels roughly near $3000 \mathrm{~m}$, where the temperature outside the rift valley is about $2.5^{\circ} \mathrm{C}$, i.e. the temperature at the level of the variations.

The temperature-salinity characteristics of deep water are modified by the plume (Figure 2.4). Most of the points in the range $2.4^{\circ} \mathrm{C}$ to $2.55^{\circ} \mathrm{C}$, lie on the warm or fresh side of a straight line representing mixing between water above the plume with water near the bottom. In the above temperature range the $\theta-S$ relation tends to the left of a mixing line owing to the influence of the plume. The amplitude of the salinity variation is about $-.004 \%$ and the amplitude of the temperature variation is about $0.02^{\circ} \mathrm{C}$. Since the salinity of the vent water is the same as the bottom water salinity, the $\theta-S$ curve of the warm buoyant plume is expected to be above the background curve, at higher temperatures. However as the plume approaches its equilibrium level, where its density anomaly disappears, it clearly cannot be both warm and fresh. Until these characteristics start to compensate, the plume has to keep rising because of its buoyancy.

The following process is suggested to explain the rise to equilibrium. The plume starts out hot and then cools by mixing, but it always remains relatively fresher than its surroundings because of its upward motion into higher salinity water. It finally rises past the level where the temperature anomaly is zero, gets heavy, and slows to a stop. Near the equilibrium there would be a range of $\theta-S$ values off the background curve but at constant density. Some evidence of this exists in the profile at station 26 (Figure 2.4). A linear approximation of isopycnals referenced to $3000 \mathrm{~m}\left(\sigma_{3}\right)$ is shown. Some of the $\theta-S$. structure is oriented approximately along isopycnals, especially in the lower part of the plume between $2.44^{\circ} \mathrm{C}$ and $2.48^{\circ} \mathrm{C}$. This tendency appears be a result of the compensating cool-fresh $\theta-S$ variation necessary near equilibrium.

Cast 24 is about $2 \mathrm{~km}$ south of cast 26 and has much reduced variations (Figure 2.5). A weak but distinct deviation is apparent in the range $2.44^{\circ} \mathrm{C}$ to $2.5^{\circ} \mathrm{C}$, which is within the temperature range of the variation in cast 26 . The magnitude of this variation is about $.02^{\circ} \mathrm{C}$. This plot can be compared with Figure 3a of Lupton et al., (1985) for a plume at the Juan de Fuca Ridge in the Pacific, in which the deviation is in the opposite sense. This comparison supports the idea that the opposite vertical salinity gradient in the two oceans may control the characteristics of the plume. The salinity effect in the Atlantic could give the plume added buoyancy, forcing it to rise to a level where it is cooler than its surroundings. A model of this process is presented in the next section.

A section was constructed by projecting some of the casts onto a line oriented approximately along the axis of the valley (Figure 2.6; Figure 1, AA'). The section is about $5 \mathrm{~km}$ long and uses 11 casts. It was constructed from casts occupied over a 


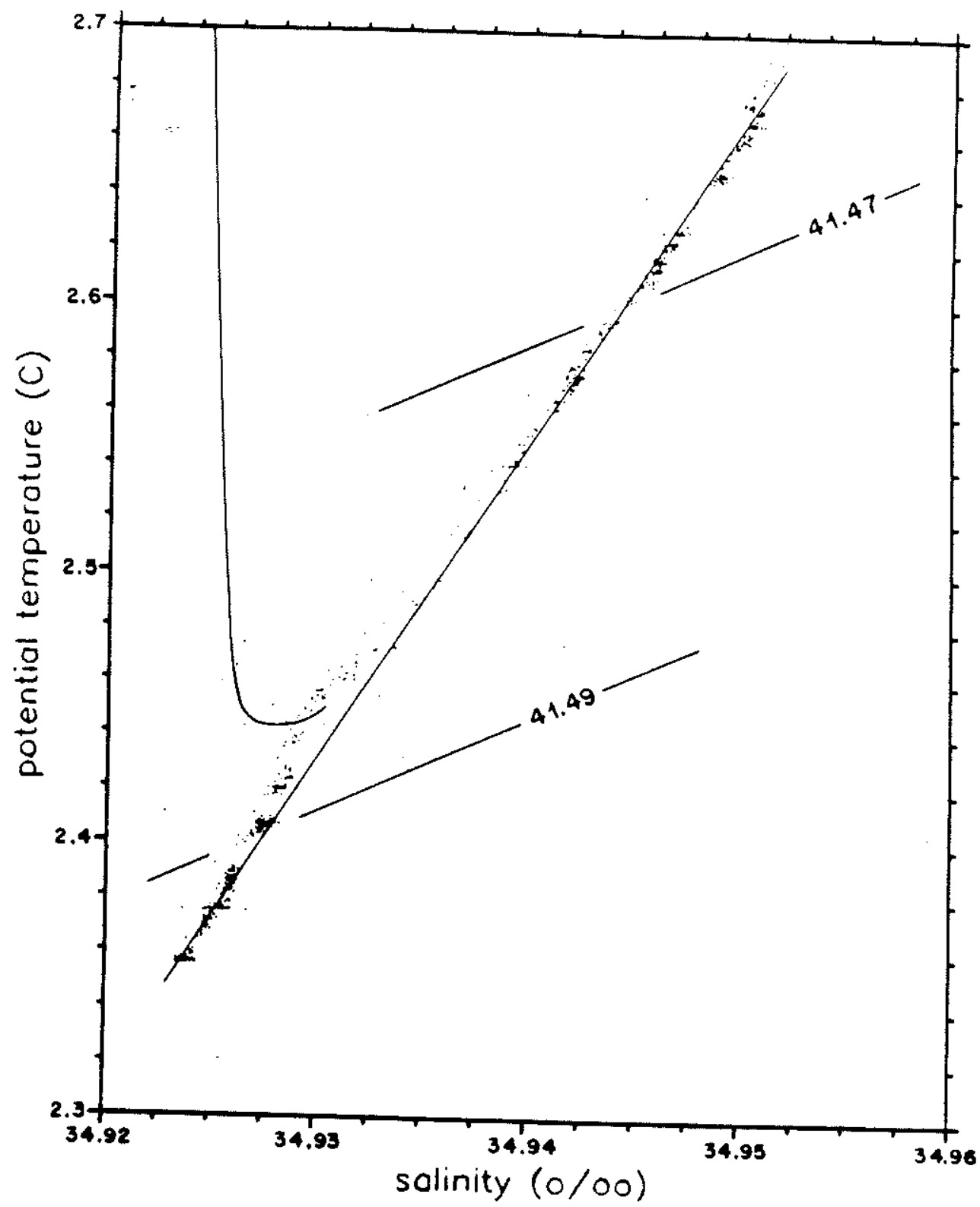

Figure 2.4: Potential temperature versus salinity, cast 26, scatter plot of $1 \mathrm{db}$ pressure sorted values. A straight line has been drawn connecting points above $3500 \mathrm{~m}$ with points near the bottom. The light solid curve is the plume model $\theta-S$ relation from Figure 2.8D (see text, section 2.4.1 and 2.5). 


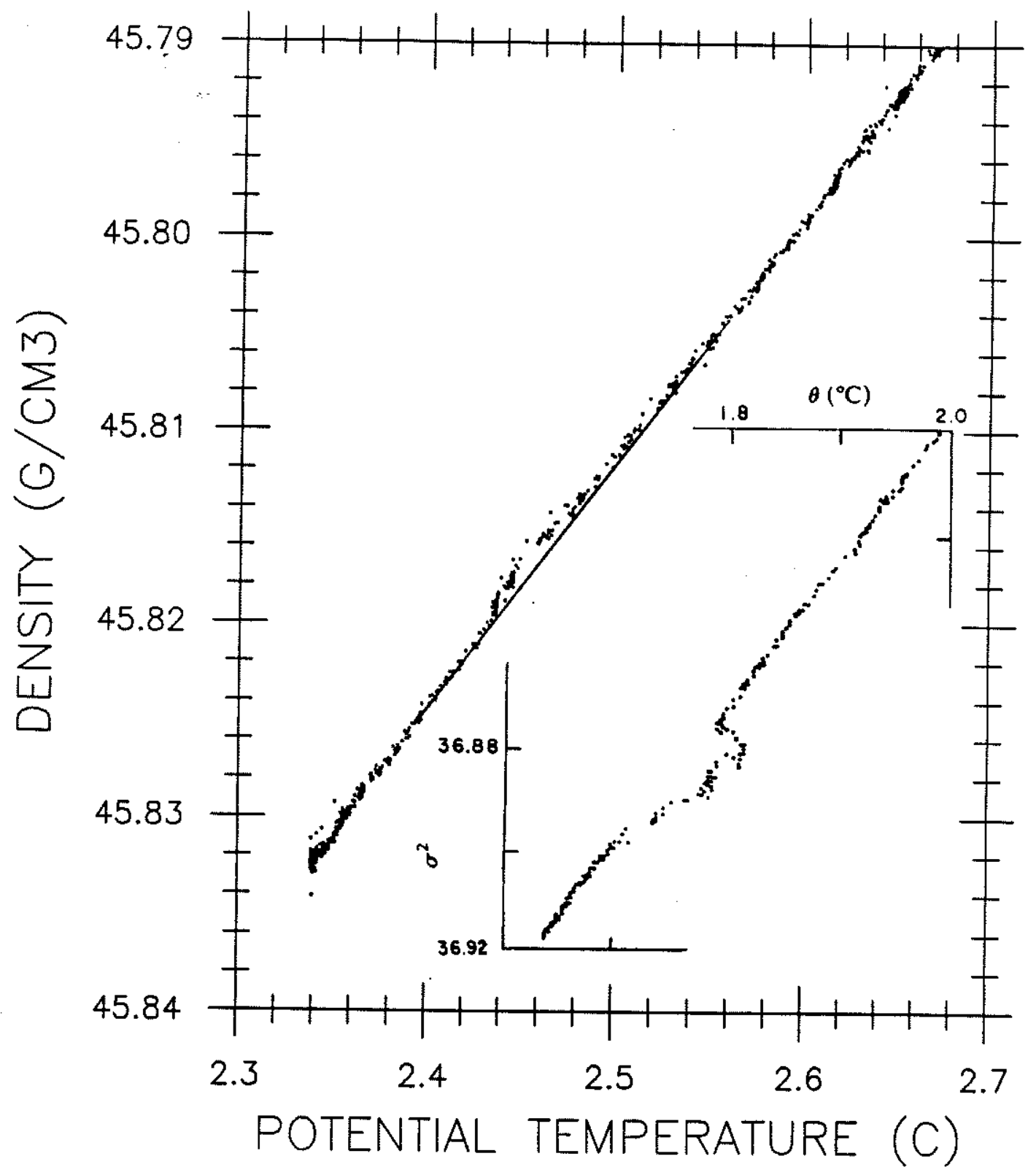

Figure 2.5: Potential temperature versus density for cast $24,2 \mathrm{~km}$ southwest of the central mound where hydrothermal activity is most intense (upper profile), and for a station from the Juan de Fuca Ridge in the Pacific (lower profile). 


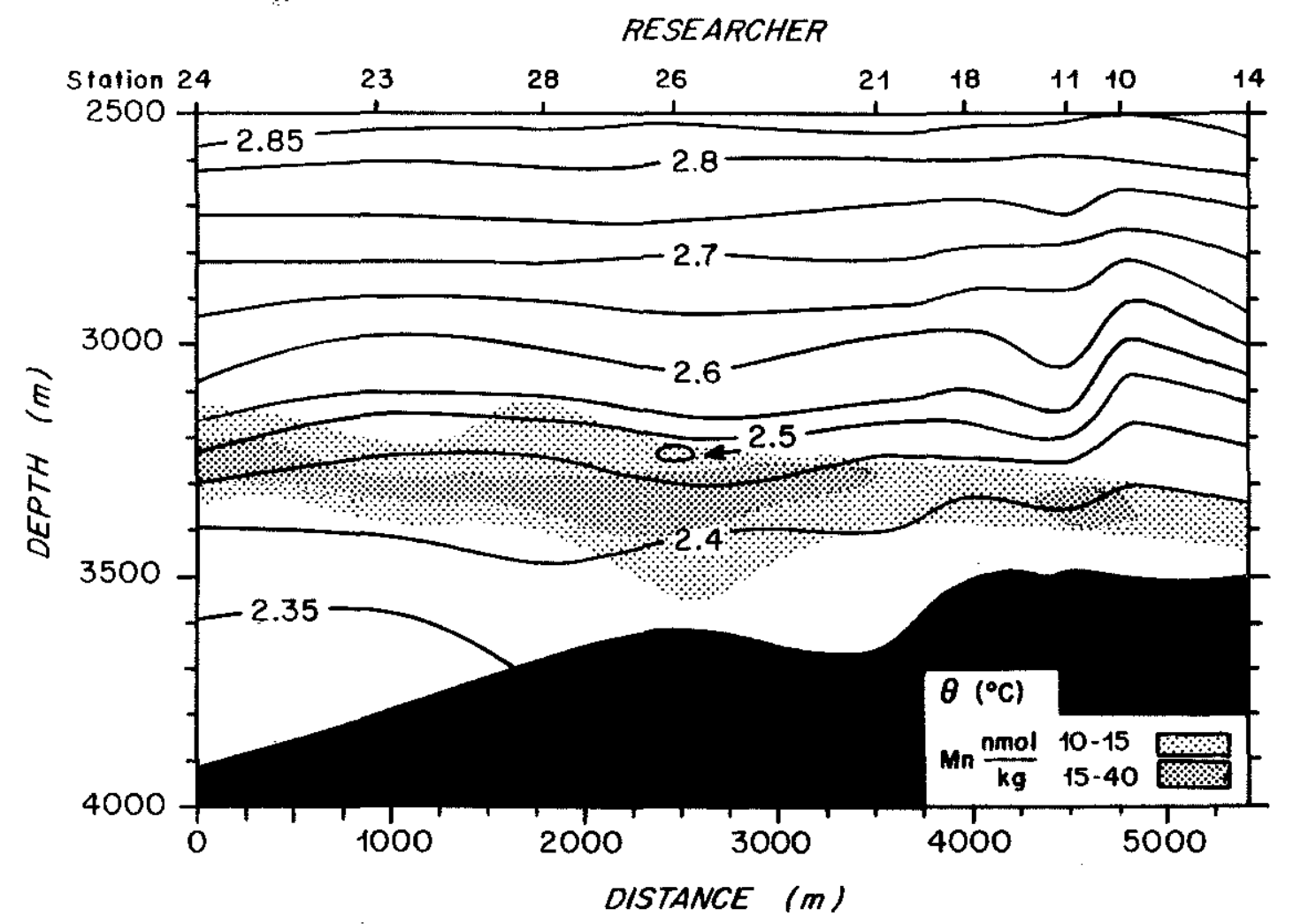

Figure 2.6: Vertical section of potential temperature, oriented parallel to the axis of the median valley (see Figure 1, AA'). An inversion exists around cast 26 near $3400 \mathrm{~m}$ depth.

period of about 2 weeks. Manganese measurements by Klinkhammer et al. (1985) are overlaid on the section to illustrate the position of the plume by its passive tracer characteristics. In general the dynamic influence of the plume, the shear and associated density field structure, are not necessarily coincident with passive tracer anomalies. There is an inversion near $3300 \mathrm{~m}$ depth at station 26 associated with the strong temperature variations within the plume.

A significant feature of the section is the correspondence between the level at which the plume spreads horizontally near $3300 \mathrm{~m}$ and an increased vertical separation of isotherms. This occurs approximately between $0 \mathrm{~m}$ and $2500 \mathrm{~m}$ horizontal distance. Such separation is characteristic of convection in stratified fluids, in which 
the forcing is strong enough to allow buoyant fluid to penetrate to some intermediate level (Morton, Taylor, and Turner, 1956; section 2.6).

Other structure in the temperature field may be associated with geostrophically balanced flow, flow affected by topography, or a sampling scheme too widely spaced in time.

The presence of active convection implies that mixing is enhanced. Little is known about mixing in deep water, and no direct measurements exist at this depth. The (finestructure) temperature Cox number which can be calculated with CTD profiles has been found to be useful in characterizing mixing elsewhere (Georgi and Schmitt, 1982). This number is the ratio of temperature variance to the mean gradient calculated over some vertical interval. The Cox number for all the TAG stations is shown (Figure 2.7). For those profiles that penetrate the plume there are strong peaks near the $45.81 \mathrm{~g} / \mathrm{cm}^{3}$ density surface. These measurements indicate that enhanced mixing occurs within the plume, and that it continues within the plume away from the source. Peaks at lower densities may represent other sources in this segment of the rift valley. It is not known how to relate these measurements to values of the cross-isopycnal mixing, but they do support the idea of increased vertical fluxes at the crest.

The magnitude of the observed Cox number near the plume is about 1000 times larger than the $\mathrm{O}(1)$ values in the thermocline reported by Georgi and Schmitt (1982). They estimate an expected value from internal waves of about 0.25 . An estimate of the heat flux into the layer from vertical diffusion is $\rho C_{p} K_{v} \Delta T A / h$, where $\rho C_{p}$ is the specific heat times density, $\Delta T=0.05^{\circ} \mathrm{C}$ is the temperature variation, $A=1 \mathrm{~km}^{2}$ is the area of the plume, and $h=100 \mathrm{~m}$ is its thickness. The vertical mixing coefficient is not known. If it lies in the range $0.1 \mathrm{~cm}^{2} / \mathrm{s}$ to $1 \mathrm{~cm}^{2} / \mathrm{s}$ outside the rift valley and is really 1000 times larger in the plume, then the heat flux is between $2 \times 10^{7} \mathrm{~W}$ and $2 \times 10^{8} \mathrm{~W}$, which must be balanced by a heat flux out of the layer owing to the plume. This range is reasonable for a typical vent source strength (section 2.4). The area is probably larger, but the total extent is not known. This method might be used to estimate source strength, and could be improved by a purposeful experimental strategy designed to sample the entire segment. The key point is that such an estimate would include both individual and diffuse sources. Until now, heat flux calculations such as those by Converse et al. (1984) have been possible only for individual high-temperature sources. 

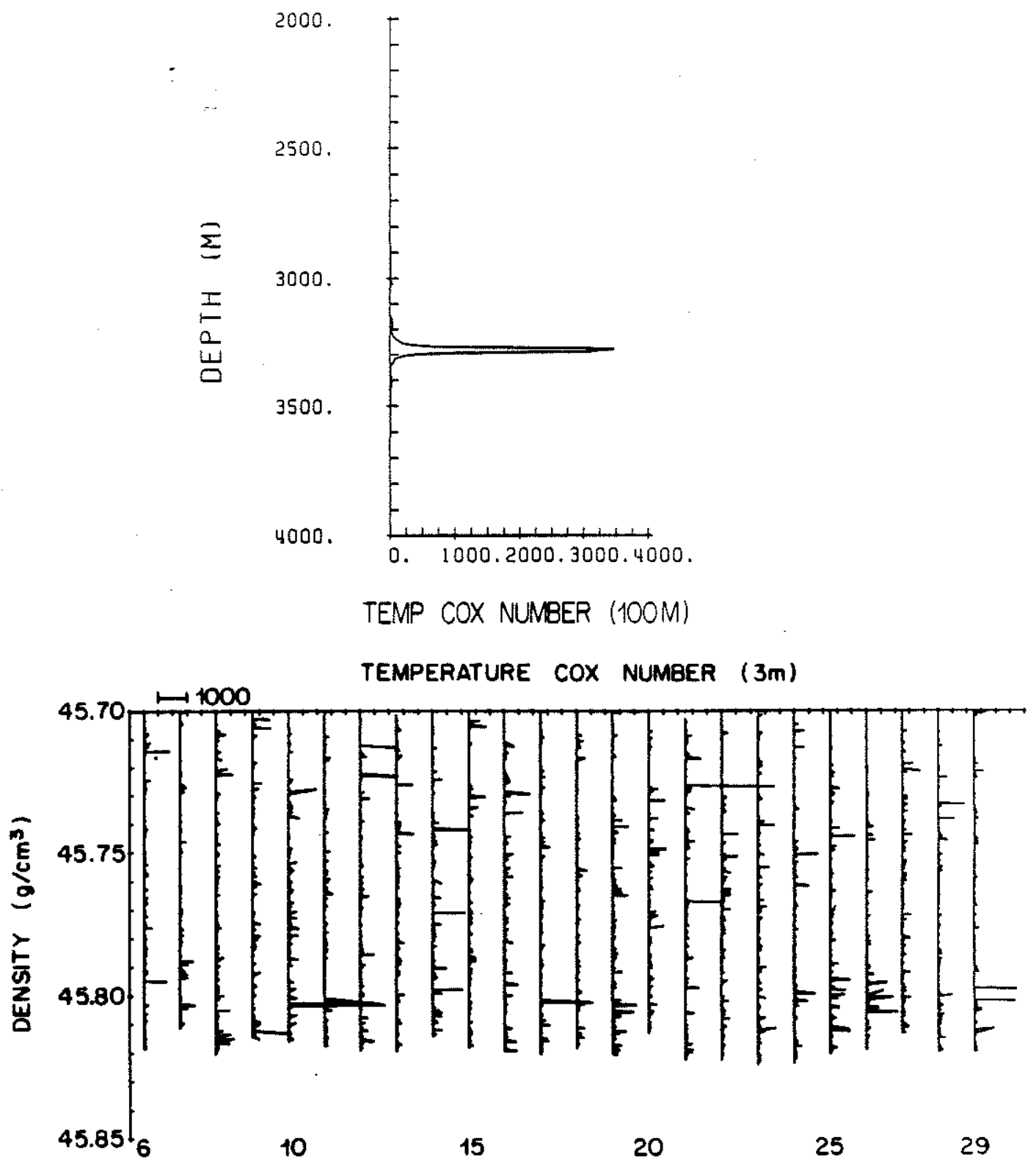

Figure 2.7: Finestructure temperature Cox number for cast 26, variance and mean taken over $100 \mathrm{~m}$ (upper). Finestructure temperature Cox number for all profiles at TAG, stations 6-29, variance and mean taken over $3 \mathrm{~m}$ (lower). Scale to 1000 shown in upper right. Density is referenced to $4000 \mathrm{db}$, and corresponds to a depth range similar to upper plot. 


\subsection{A turbulent plume model and comparison to observa- tions}

A model was used to calculate the behavior of a turbulent plume in the Atlantic and compare it to one in the Pacific. The model is based on the physics discussed by Morton, Taylor and Turner (1956), with an entrainment constant fixed at .072 (their $\alpha$ ). There is a point source of buoyant fluid on a flat bottom. The plume rises through a stably stratified background characterised by the buoyancy frequency $\mathrm{N}$ (see below). Rotation can be neglected because in all cases considered here, the time scale of the plume $\mathrm{N}^{-1}$ is fast compared to that of rotation, $\mathrm{f}^{-1}$. This time-scale separation is part of the definition of the small-scale regime discussed in Chapter 1.

The variables in the model are area (A), vertical velocity $(\mathrm{W})$, potential temperature $(\Theta)$, and salinity $(S)$ of the plume. These are average quantities resulting from a horizontal integration across the plume, and are calculated as a function of height $z$ above bottom. These quantities vary with height because of mixing, which is represented in the model by entrainment. The basic assumption is that the entrainment into the plume is proportional to the velocity. The conservation equations for mass, salt, heat, and momentum are

$$
\begin{gathered}
(A W)_{z}=E W \\
(S A W)_{z}=\bar{S} E W \\
(\Theta A W)_{z}=\bar{\Theta} E W \\
\left(\rho_{o} A W^{2}\right)_{z}=g(\rho-\bar{\rho}) A
\end{gathered}
$$

where $\mathrm{g}$ is gravity and the overbar denotes the background field. The entrainment coefficient is $\mathrm{E}=0.072$.

The new feature is the consideration of temperature and salinity separately to allow the calculation of the $\theta-S$ properties of the plume. An equation of state must be specified. The linear approximation $\rho=1.041548-2.13 \times 10^{-4}(\Theta-2.0)+$ $7.5 \times 10^{-4}(S-34.89)$ was thought to be adequate over most of the range of salinity, temperature, and pressure of the plume. An equation of state valid over the entire range of these variables encountered in the plume is not known. For instance, the effect of salt variations at high temperatures on the equation of state is not well known. As discussed by Little et al. (1987), a nonlinear equation of state is appropriate at the high initial temperatures of the plume. However, the differences between runs with the nonlinear equation of state and those using a linear one were relatively small, about $20 \%$. Because of this situation, and also the exploratory nature of the data set, the model here is kept in its simplest form. Measurements of velocity and temperature at several heights in a buoyant plume on the Juan de 
Fuca Ridge in the Pacific were found by Little et al. (1987) to be consistent with a turbulent plume model.

The background potential temperature $(\bar{\Theta})$ and salinity $(\bar{S})$ are specified linear functions of height. As the plume rises it entrains water with properties appropriate for each level, given by the barred fields. The Atlantic background profile is $\bar{\Theta}=$ $2.335+4 \times 10^{-4} z, \bar{S}=34.923+4 \times 10^{-5} z$. The Pacific background profile is $\bar{\Theta}=$ $1.80+10^{-3} z, \bar{S}=34.608-10^{-4} z$. The Pacific values are taken from Lupton et al. (1985). The initial conditions for area and velocity were chosen by estimating values from a video recording of a vent system outside the central mound at the TAG area. Temperature and salinity initial conditions were obtained by measurement. The initial conditions are $A=0.1 \mathrm{~m}^{2}, \mathrm{~W}=40 \mathrm{~cm} / \mathrm{s}, \Theta=300^{\circ} \mathrm{C}, \mathrm{S}=34.923 \%$ (Atlantic), $\mathrm{S}=34.608 \%$ (Pacific). A Runge-Kutta fourth order routine was used to integrate the equations.

The calculation proceeds until the velocity of the plume drops to zero, at which point the plume has reached its penetration height $z^{*}$. The plume must pass the equilibrium level where the density difference in zero, in order for negative buoyancy to decelerate the flow. In this model the penetration height satisfies

$$
z^{*}=5 B o^{\frac{1}{4}} N^{\frac{-3}{4}}
$$

(Turner, 1973) where the buoyancy flux $B o$ and buoyancy frequency $N$ are given by

$$
\begin{gathered}
B o=g(\alpha(\Theta-\bar{\Theta})-\beta(S-\bar{S})) A W \\
N^{2}=-g\left(\alpha \bar{\Theta}_{z}+\beta \bar{S}_{z}\right) / \rho
\end{gathered}
$$

The variables in these expressions are evaluated at $z=0$. The expansion coefficients $\alpha$ and $\beta$ come from the equation of state. The value of $B o$ in both cases is $2.36 \times 10^{-2} \mathrm{~m}^{4} / \mathrm{s}^{3}$ and the values of $N$ are $7.35 \times 10^{-4} \mathrm{~s}^{-1}$ (Atlantic), $1.68 \times 10^{-3} \mathrm{~s}^{-1}$ (Pacific). The slightly different initial temperature differences $\Theta-\bar{\Theta}$ in the two cases has a negligible effect on the buoyancy flux.

\subsubsection{Atlantic case}

Several basic and derived variables are illustrated in the solution for the Atlantic case (Figure 2.8). In the model, the Atlantic plume rises $330 \mathrm{~m}$. This height compares favorably with the TAG observations of about a $350 \mathrm{~m}$ penetration. A relatively low value of $\mathrm{N}$ at the TAG area results in a penetration height there which is greater than other known plumes. It is still well below the level of the mountains on either side of the rift valley, which rise $1700 \mathrm{~m}$ above the bottom. The plume continues rising $100 \mathrm{~m}$ past its equilibrium level before coming to a stop. Mixing 
occurring in this region tends to spread out the plume over a thick layer, and so has important observational consequences by reducing the amplitude of anomalies. Throughout most of the vertical extent of the plume the velocity is about $10 \mathrm{~cm} / \mathrm{s}$ (Figure 2.8A). This is strong enough to keep particles suspended and is consistent with the measurement of particulates by the CTD at the equilibrium level. With this velocity, the time scale for the plume to reach the equilibrium height is about $1 \mathrm{hr}$, which is similar to the buoyancy frequency time $N^{-1}$ of about $1 / 2 \mathrm{hr}$. Near $200 \mathrm{~m}$ height the temperature of the plume equals that of the background (Figure $2.8 \mathrm{~F}$ ). Past this level the buoyant force comes from the salinity deficit in the plume (Figure 2.8E). Salinity in the plume diverges from the background because of the upward motion of fresh water mixed in from deeper levels. The resulting anomaly around the equilibrium level is cool and fresh (Figure 2.8D).

The entrainment and vertical transport are calculated in the model. The net entrainment is the ratio of final to initial area and for the Atlantic case is $2.4 \times 10^{8}$. The vertical transport past the equilibrium level into the layer is $632 \mathrm{~m}^{3} / \mathrm{s}$, (about 3 times the Pacific value). The volume of the $10 \mathrm{~km}$ by $10 \mathrm{~km}$ segment below $330 \mathrm{~m}$ can be divided by this transport to give a recycling time of about 1.5 yrs. Another way to illustrate this transport is to convert it into a vertical velocity. If, for example, there were 1 vent every $2 \mathrm{~km}$ on average along an active part of the crest $10 \mathrm{~km}$ by $1000 \mathrm{~km}$, then the average upward velocity would be $3 \times 10^{-4} \mathrm{~cm} / \mathrm{s}$. There is no buoyancy flux into the layer by definition, since $\Delta \rho$ is zero, but there are compensating heat and salt fluxes. The heat flux is $\rho C_{p} \Delta T A W=-5 \times 10^{7} \mathrm{~W}$ (heat loss) and the salt flux is $\rho \triangle S A W=2.5 \times 10^{3} \mathrm{~g} / \mathrm{s}$. This heat flux value lies in the range of values from the Cox number estimate in section 2.3 .

\subsubsection{Pacific case}

The penetration height in the Pacific case is $180 \mathrm{~m}$ (Figure 2.9), which agrees with the observations of Lupton et al. (1985), which are close to $200 \mathrm{~m}$. The stronger background stratification (higher $\mathrm{N}$ ) in the Pacific results in the plume rising less far past its equilibrium level than in the Atlantic. If a layer thickness is defined as the difference between the equilibrium height and final height, the layer is twice as thick in the Atlantic as in the Pacific (Figures 2.8B, 2.9B). Thus $\theta-S$ anomalies are spread out over a larger volume in the Atlantic, reducing their amplitude compared to the Pacific. However both the Atlantic and Pacific plumes appear in observations to have a thickness of about $150 \mathrm{~m}$. Perhaps a more realistic background stratification (nonlinear) would result in similar thicknesses. The velocity overall is about $10 \%$ higher in the Pacific case, resulting from a combination of faster time scale (higher $\mathrm{N}$ ) yet smaller penetration height $z^{*}$. Again, throughout most of the vertical extent 


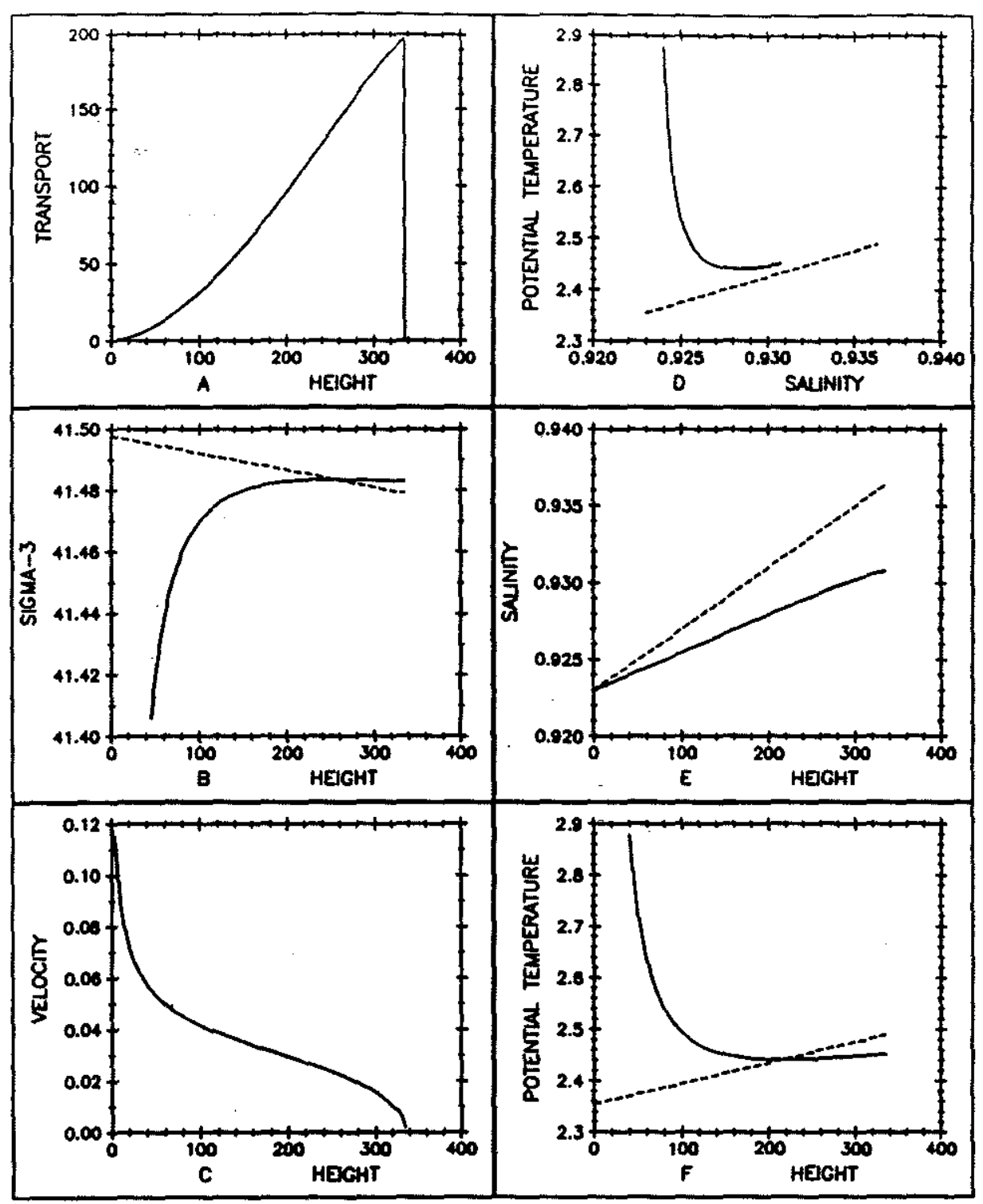

Figure 2.8: Solution for Atlantic case. Solid lines are the plume, dashed lines are the background. Height in meters. (A) Transport (scaled by $3.16 \mathrm{~m}^{3} / \mathrm{s}$ ). (B) Density $\left(\mathrm{g} / \mathrm{cm}^{3}\right)$ referenced to $3000 \mathrm{db}$. The axis scale is set to visualize the approach to equilibrium and so the very light fluid near $z=0$ is off-scale. (C) Velocity (scaled by $3.16 \mathrm{~m} / \mathrm{s}$ ). (D) $\theta-S$ relation for plume and background $\left({ }^{\circ} \mathrm{C}\right.$ and $\%$ ). (E) Salinity-34\%. (F) Temperature $\left({ }^{\circ} \mathrm{C}\right)$. 


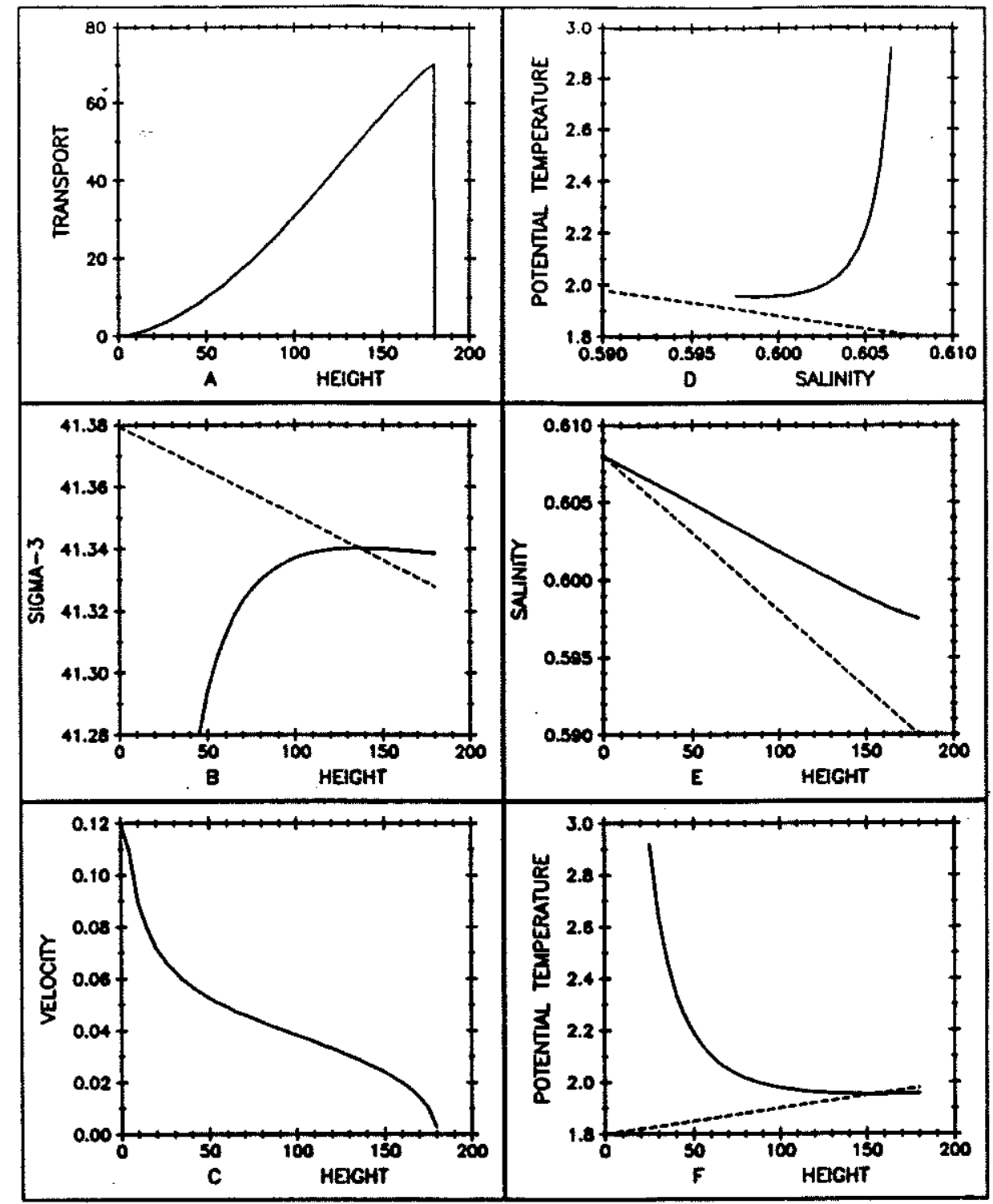

Figure 2.9: Solution for Pacific case. Solid lines are the plume, dashed lines are the background. Height in meters. (A) Transport (scaled by $3.16 \mathrm{~m}^{3} / \mathrm{s}$ ). (B) Density $\left(\mathrm{g} / \mathrm{cm}^{3}\right)$ referenced to $3000 \mathrm{db}$. The axis scale is set to visualize the approach to equilibrium and so the very light fluid near $z=0$ is off-scale. (C) Velocity (scaled by $3.16 \mathrm{~m} / \mathrm{s}$ ). (D) $\theta-S$ relation for plume and background $\left({ }^{\circ} \mathrm{C}\right.$ and $\%$ ). (E) Salinity-34\% . (F) Temperature $\left({ }^{\circ} \mathrm{C}\right)$. 
of the plume the velocity is about $10 \mathrm{~cm} / \mathrm{s}$. The entrainment is $7.7 \times 10^{7}$, and the transport past the equilibrium level into the layer is about $200 \mathrm{~m}^{3} / \mathrm{s}$.

\subsection{Discussion}

Of special interest are the $\theta-S$ characteristics of the two plumes (Figure 2.8D and 2.9D). In the Pacific the stable background salinity gradient means that relatively warm salty water will spread at the equilibrium level. In the Atlantic, the unstable background salinity gradient means that the plume must continue to rise until it is cool compared to its surroundings, to come to equilibrium. A significant qualitative correspondence exists between the model $\theta-S$ results (Figure 2.8D) and the observed $\theta-S$ variation (Figure 2.4). Below $2.54^{\circ} \mathrm{C}$, and especially below $2.48^{\circ} \mathrm{C}$, the observed $\theta-S$ relation moves off a mixing line approximately along isopycnals in the cool, fresh direction. Below $2.42^{\circ} \mathrm{C}$, the observed $\theta-S$ relation merges with bottom values which are nearly in line with those above the layer. This pattern is the same as that implied by the model, since the final plume $\theta-S$ values lie on the fresh side of the background $\theta-S$ line (Figure 2.8d). The model $\theta-S$ curve (Figure 2.8d, solid line) is plotted together with the observations in Figure 2.4 to illustrate this tendency. In the ocean these different characteristics mix approximately along isopycnals. Therefore the plume near equilibrium acts as a cool, fresh source in a limited density range shifting the $\theta-S$ relation away from the linear background relation. Figure 2.5 nicely illustrates the different nature of the plumes in the two oceans, resulting from the opposite vertical salinity gradient.

The structure of buoyant plumes in the Atlantic and Pacific is illustrated schematically in figure 2.10. It has been assumed that the vent water has the same salinity as the surrounding water at the exit level. For those vents with an initial salinity flux as well, vertical entrainment would rapidly bring the vent water close to ambient values, as happens with temperature. The plume would continue to entrain fluid, and eventually lag the background values as it rises to a new penetration height given by the new buoyancy flux $B_{0}$ which includes the contribution from salinity. The equilibrium anomalies have to compensate in the same manner, but their magnitude is increased or decreased somewhat depending on the new penetration height.

The entrainment of ambient seawater into the plume, and the upward motion of this water to lower densities generates, in the equilibrium layer, anomalous values of any quantity with significant vertical variation over the height scale of the plume. For example, the vertical gradient of silica in the depth range $2500 \mathrm{~m}$ to $3500 \mathrm{~m}$ near the TAG site is about $-10 \mu \mathrm{mol} / 1 / \mathrm{km}$. If silica from a given level is mixed upwards $300 \mathrm{~m}$ an anomaly of several $\mu \mathrm{mol} / 1$ can result. This assumes that the 

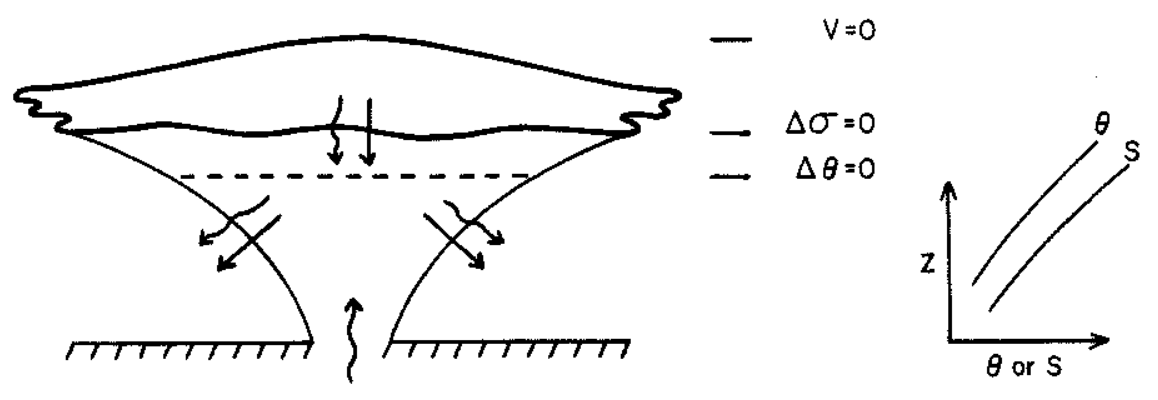

ATL ANTIC
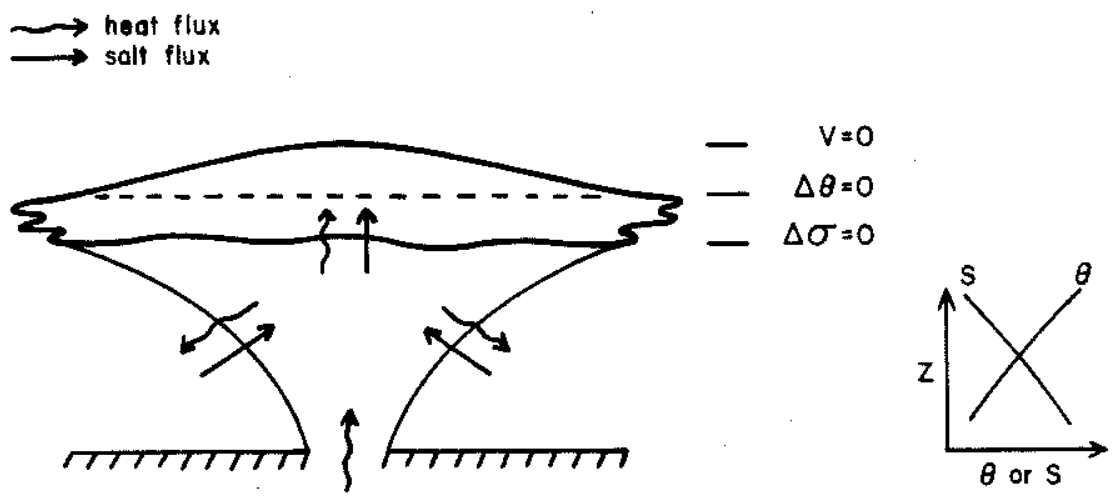

PACIFIC

Figure 2.10: Schematic of temperature and salinity fluxes for buoyant plumes in the Atlantic and the Pacific, which have opposite background salinity profiles. 
source of silica to the plume from vent water is negligible compared to the source from entrainment, which may not always be the case. In general, the change in the average concentration of any quantity over the area of the plume with height above bottom depends on the initial concentration and background profile through the physics of the entrainment process. Therefore the final equilibrium concentration also depends on the entrainment process and cannot be calculated as the result of simple two-point mixing between source water and ambient water.

The observed properties are not easily related to model variables, making estimates of mixing and fluxes from observations unrepresentative of the important net quantities. The key point is that individual CTD profiles do not represent averages over the turbulent fields within the plume. Furthermore the situation of a buoyant plume rising in a variable background state does not correspond to simple dilution of $\theta$ or $\mathrm{S}$, to allow an estimate of entrainment or heat flux solely from observed equilibrium anomalies. This is especially clear in the Atlantic where the equilibrium temperature anomaly is negative. To estimate the entrainment and associated vertical transport of water an account must be kept of the mixing occurring throughout the plume as it rises, for example by constructing a vertical profile of horizontally averaged salinity in the plume. Salinity measurements in the spreading layer do not give information about the level from which the entrained water came. To measure heat flux the net divergence of heat in a box surrounding the source must be calculated. This quantity is usually not resolved because it is the difference between large quantities subject to significant error. Until such measurements are performed, combining existing data with simple models to estimate mixing and fluxes seems most appropriate and informative.

\subsection{Horizontal Flow: a 2-d Forced Baroclinic Vortex}

In the Pacific, isotherm deviations of several hundred meters are observed within about $10 \mathrm{~km}$ of heat sources, corresponding to horizontal temperature variations of about $0.1^{\circ} \mathrm{C}$ (Crane et al., 1985). Some of this structure is probably associated with horizontal geostrophic flow, but this possibility has not been discussed in the past. The turbulent plume model discussed in the previous section allows the vertical structure of the buoyant plume to be calculated. Implicit in the entrainment process in this model was a background flow coming in from an infinite distance to supply the necessary mass. This flow is discussed by Turner (1973) for a system which is not rotating. Rotation effects could be neglected in the plume model because the time-scale for a parcel of fluid to rise in the plume is small compared to an inertial period (one hour compared to 10 hours). As fluid spreads near its equilibrium level the time scales become comparable and rotation cannot be neglected. Rotation 
has an important effect on the background flow as fluid can no longer move in from infinite distance because that would violate angular momentum conservation. From simple ideas about the tendency of rotation to cause flow to turn, a recirculation and trapping of fluid near the source might be anticipated. In this section the general form of the solution to linear f-plane flow forced by an internal buoyancy source is shown, following Gill (1982). Unlike his example, which was for a special form of the forcing, simple analytic forms for the solution do not exist because the forcing calculated in the turbulent plume model has a complex vertical structure.

Gill's (1982) model was time-dependent and inviscid, and was supposed to represent the initial stage of the response to buoyancy forcing. The amplitude of his solution increased with time, and since the model was linear, friction eventually became important. Friction and diffusion were represented by Rayleigh damping, where the time derivative is replaced by a friction parameter $r$ to get a steady solution. This technique is used here also for simplicity. The other simplification is the neglect of the nonlinear terms. These neglected terms are small for weak enough forcing. Their size for a given strength forcing is estimated below.

The crest of the East Pacific Rise is several tens of kilometers wide, while the median valley is a depression only a few hundred meters deep. Thus the simplifying assumption of a flat bottom is probably valid in this situation. The rift valley of the Mid-Atlantic Ridge is of similar width, but the mountains on either side typically rise $1000 \mathrm{~m}$ to $2000 \mathrm{~m}$ above the floor of the valley. In this case topography could be important, depending on the exact location of the source within the valley. For this study, the bottom is assumed to be flat. The domain is the infinite half-plane $z>0$, $-\infty<x<\infty$, with $z=0$ at the bottom sitting on top of a crest of a north-south mid-ocean ridge. The $x$-axis is aligned in the east-west direction. This domain is only meant to represent the crest, not the entire ridge.

The equations are:

$$
\begin{gathered}
r u-f v=-P_{x} \\
r v+f u=0 \\
P_{z}=-g \rho / \rho_{o} \\
r \rho+w \rho_{z}=\frac{-B}{g} \\
u_{x}+w_{z}=0
\end{gathered}
$$

where $r \ll f$ is the Rayleigh damping and B is the internal buoyancy source, localized at $x=0$. The boundary conditions are $w=0$ at $z=0$ and decay away from the source. The basic balances away from the source are that vertical motion forces isopycnals to move (2.4 without $B$ ), giving rise to horizontal pressure gradients using the hydrostatic relation (2.3). The resulting flow along the ridge, $v$, 
is mostly geostrophic (2.1), while the frictional drag it experiences (2.2) is balanced by the Coriolis force from an $\mathrm{O}(r)$ weaker cross-ridge flow, $u$.

A streamfunction can be defined $\psi_{x}=w$ and $\psi_{z}=-\mathrm{u}$. The equation for $\psi$ is

$$
\psi_{x x}+\frac{f^{2}+r^{2}}{N^{2}} \psi_{z z}=\frac{B_{x}}{\rho_{o} N^{2}}
$$

where $N^{2}=-g \rho_{z} / \rho_{0}$. In the following $N$ will be constant. Writing

$$
\begin{aligned}
& \psi(x, z)=\int_{0}^{\infty} \hat{\psi}(x, m) \sin (m z) d m \\
& B(x, z)=\int_{0}^{\infty} \hat{B}(x, m) \sin (m z) d m
\end{aligned}
$$

and substituting gives an equation for $\hat{\psi}$ with solutions exponential in $\mathrm{x}$. Integrating the $\hat{\psi}$ equation from $\mathrm{x}=0$ to $\mathrm{x}=\infty$ gives a condition which fixes the amplitude of the exponential solution in terms of $\hat{B}$. Thus

$$
\psi(x, z)=\int_{0}^{\infty} \frac{\operatorname{sgn}(x) \hat{B}(m)}{2 f m N \rho_{o}} e^{\frac{-f m|x|}{N}} \sin (m z) d m .
$$

The forcing $\hat{B}$ comes from the turbulent plume model calculation of the vertical structure of the plume. The entrainment into the plume causes a convergent flow just outside the plume boundary. The buoyancy source generates the upwelling which balances this convergent flow. This idea can be used to calculate B. At the source the dominant balance is

$$
w N^{2}=B / \rho
$$

Substituting into the mass equation (2.5) gives

$$
\frac{\partial}{\partial z}\left(\frac{B}{\rho N^{2}}\right)=-\frac{\partial u}{\partial x}
$$

The right hand side is just the inflow divided by the horizontal length scale of the plume. This length scale represents the distance over which the inflow turns towards the vertical direction, and will be taken to be the final radius of the plume $\left(A_{f} / \pi\right)^{1 / 2}$. Using the entrainment assumption which relates the inflow to the plume velocity $\mathrm{W}$, the above equation becomes

$$
\frac{\partial}{\partial z}\left(\frac{B}{\rho N^{2}}\right)=\frac{E W}{A_{f}}=\frac{(A W)_{z}}{A_{f}}
$$

So that

$$
B=\frac{A W}{A_{f}} \rho N^{2}
$$


This expression for $B$ is in terms of quantities calculated in the plume model, and thus can be Fourier transformed and substituted into the equation for $\psi(2.7)$. For $W=10 \mathrm{~cm} / \mathrm{s}$ and $N=10^{-3} \mathrm{~s}^{-1}, B=10^{-5} \mathrm{~cm} / \mathrm{s}^{3}$. The area factor makes $B$ vary from about $10^{-7} \mathrm{~cm} / \mathrm{s}^{3}$ to $10^{-6} \mathrm{~cm} / \mathrm{s}^{3}$ throughout most of its vertical range. The corresponding scale for horizontal flow is $u=\frac{B}{\rho_{0} f N}$, which for $f=10^{-4} \mathrm{~s}^{-1}$ and $N=10^{-3} \mathrm{~s}^{-1}$ is about $10 \mathrm{~cm} / \mathrm{s}$. This is sufficiently strong to overcome background flow of order $1 \mathrm{~cm} / \mathrm{s}$, and allow recirculation. The scale for the vertical velocity outside the plume is $B / N^{2}$, or about $1 \mathrm{~cm} / \mathrm{s}$.

The length scale in equation 2.10 is not an exact factor, but may be calibrated by the condition

$$
\int_{V} B d V=F_{0}
$$

that is, the integral of the buoyancy flux divergence over all space equals the buoyancy source $F_{0}$ at the bottom.

The main characteristics of the forcing are that it is vertically confined to a distance less than the penetration height $z^{*}$ above the bottom, and that it is nonzero at the bottom. Since $w=0$ at the bottom, a vertical pressure gradient (and therefore a density anomaly) exists to balance the buoyancy force there.

To illustrate the solution it is easier in practice to solve the Poisson equation (2.6) numerically. This equation can be nondimensionalized by choosing $\psi \propto$ $B_{0} L / \rho_{0} N^{2}$, and $L \propto N z^{*} / f$, where $B_{0}$ is the scale of the forcing. A solution is illustrated for forcing which has a vertical structure like that of the vertical transport in the plume model (Figure 2.11). Flow converges into the source region $(z=0$ to $z=1$ ), rises, and exits in a thin layer just above the top of the source. This circulation closes by downwelling over a region of radius $L$. A small amplitude streamline (0.05) indicates that the total extent of downwelling is at least twice as big. The meridional flow, $v$ (scaled by $B_{0} / \rho_{0} r N$ ), is in the clockwise sense above and counterclockwise sense below.

The scale for the density perturbations is $B_{0} / g r$, which is about $10^{-4} \mathrm{~g} / \mathrm{cm}^{3}$ for $r=10^{-5} \mathrm{~s}^{-1}$. Isopycnals are pushed down where there is downwelling and lifted up where there is upwelling. At the top of the source, a weak inversion exists which goes away if $r$ is increased slightly. In this region, vertical stability is reduced.

The pattern of flow illustrated for a point source in two dimensions can be generalized to three dimensions. Above about $z=0.9$, where the flow is away from the source, anticyclonic motion exists. Below this level, where flow is convergent, cyclonic motion exists. Thus isopycnals spread apart near $z=1.1$ consistent with the thermal wind. In terms of a geostrophic calculation moving down from an upper level of no motion, the tilting isopycnals generate flow which is reversed in the lower part of the circulation cell. 


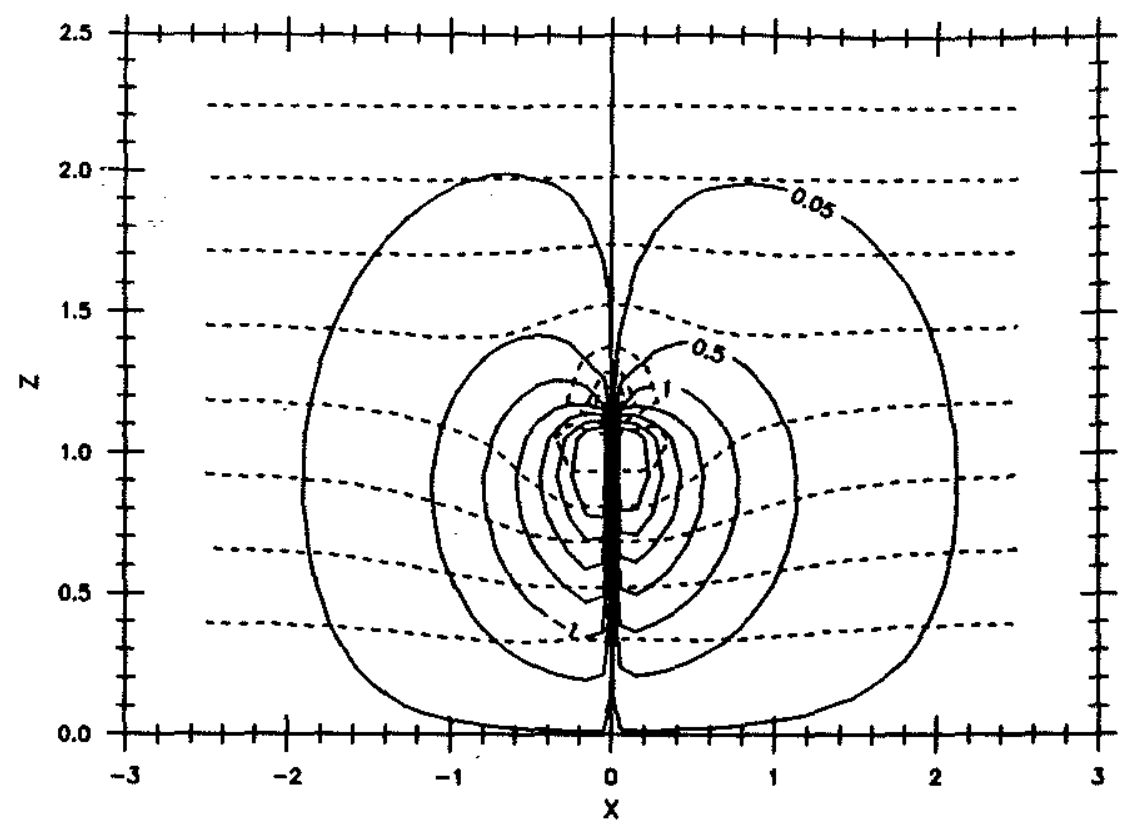

A

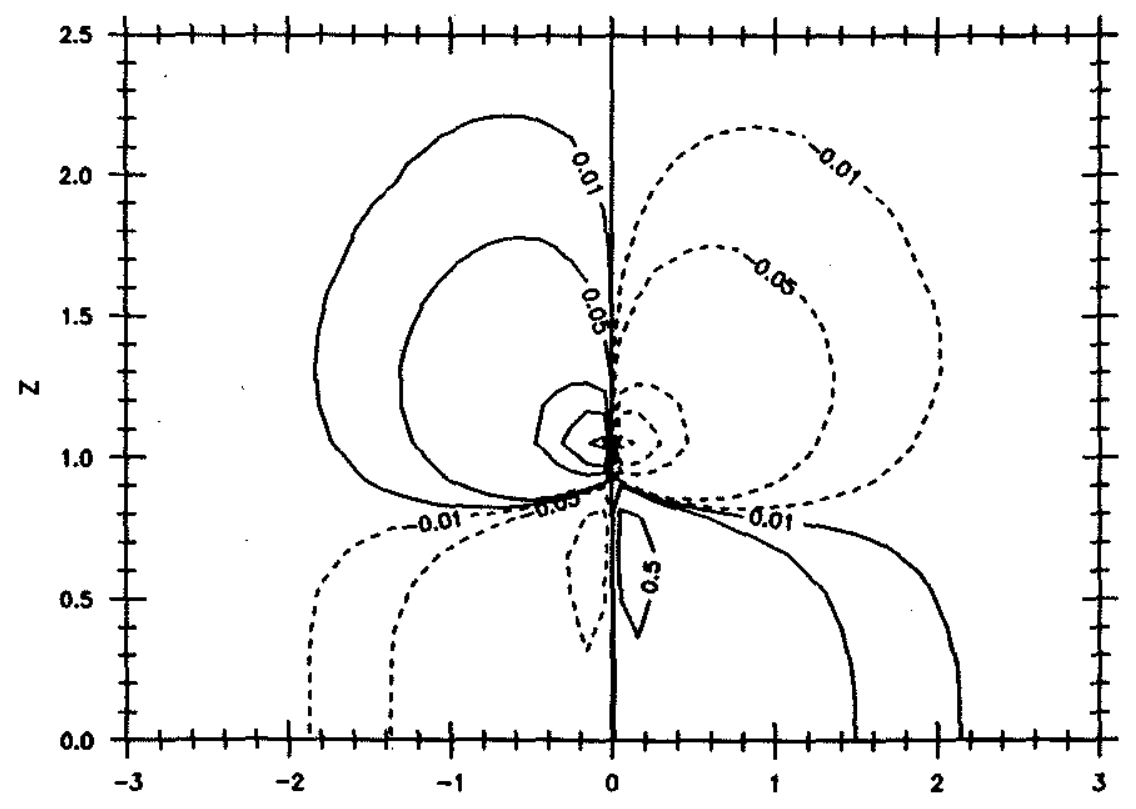

Figure 2.11: A) Streamlines (solid) and density perturbations (dashed) of vortex forced by turbulent plume. The forcing is located at $x=0, z=0$. Height is scaled by $z^{*}$ and width is scaled by $N z * / f$. B) Meridional velocity $v$, positive values are northward. 
The size of the Rossby number $R=U / f L$ measures the importance of the nonlinear terms compared to the Coriolis force in equations 2.1, 2.2. Substituting the above scales gives $R=\frac{B}{f N^{2} z^{*}}=0.3$ for $B=10^{-6} \mathrm{~cm} / \mathrm{s}^{3}, N^{2}=10^{-6} \mathrm{~s}^{-1}, f=$ $10^{-4} \mathrm{~s}^{-1}$, and $z^{*}=3 \times 10^{4} \mathrm{~cm}$. In the density equation the ratio $\frac{u \rho_{x}}{w \rho_{z}}$ is $U / r L$, using 2.4 with $B=0$. This ratio is 3 for the values used above. The nonlinear terms are important, at least close to the source where $u$ is largest, so the linear model is strictly valid if $B$ is smaller or the stratification is stronger. Numerical solutions to equations similar to 2.1-2.5 but including nonlinear heat advection and planetary $\beta$ $\left(\frac{\partial f}{\partial y}\right)$ are examined in Chapter 4.

Some observational support for this circulation appears in recent measurements of a hydrothermal plume at the Juan de Fuca Ridge (Figure 2.12). The strong chemical anomalies indicate that this plume resulted from a sudden emptying of a hydrothermal system (Baker, Massoth, and Feely, 1987). The important feature of the cross-section of the plume is the vertical spreading of isopycnals centered near $1800 \mathrm{~m}$ depth and $12 \mathrm{~km}$ distance, whose pattern is similar to the model density deviations.

The penetration height of the plume is $700 \mathrm{~m}$, to about $1800 \mathrm{~m}$ depth, which is 3.9 times the penetration height of the example in the previous section. According to the formula $z^{*}=5 B_{o}^{1 / 4} N^{3 / 4}$ the buoyancy source would be $3.9^{4}$ or about 230 times greater. Such a strong source probably has nonlinear effects not present in the linear model of the horizontal flow. The observed density deviations, however, would be associated with strong but not huge geostrophic flow. The geostrophic flow corresponding to a horizontal density deviation of $2.0 \times 10^{-5} \mathrm{~g} / \mathrm{cm}^{3}$ over a horizontal scale of $5 \mathrm{~km}$ and vertical scale of $500 \mathrm{~m}$ amounts to about $20 \mathrm{~cm} / \mathrm{s}$. This example and other observations (e.g. Crane et al., 1985) of the structure of the background field above a ridge crest suggest that the main effect of the turbulent plume on its environment may be represented by a buoyancy driven vortex. Evidence for isolated patches of order $10 \mathrm{~km}$ in diameter from chemical tracer measurements is increasingly common as more complete sampling programs are conducted near ridges, and it may be speculated that nonlinear effects can produce isolated eddies from a continuous buoyancy source at the crest.

\subsection{Large-scale $\theta-S$ anomalies above the Mid-Atlantic Ridge}

Measurements of helium-3 close to the TAG hydrothermal field show values elevated above background, indicating a local injection (Jenkins, Rona, and Edmond, 1980). Deep water helium data from the Transient Tracers observational program with sparse but adequate coverage in the North Atlantic and tropical Atlantic to justify looking for a large-scale plume were provided by W. Jenkins (this program was 


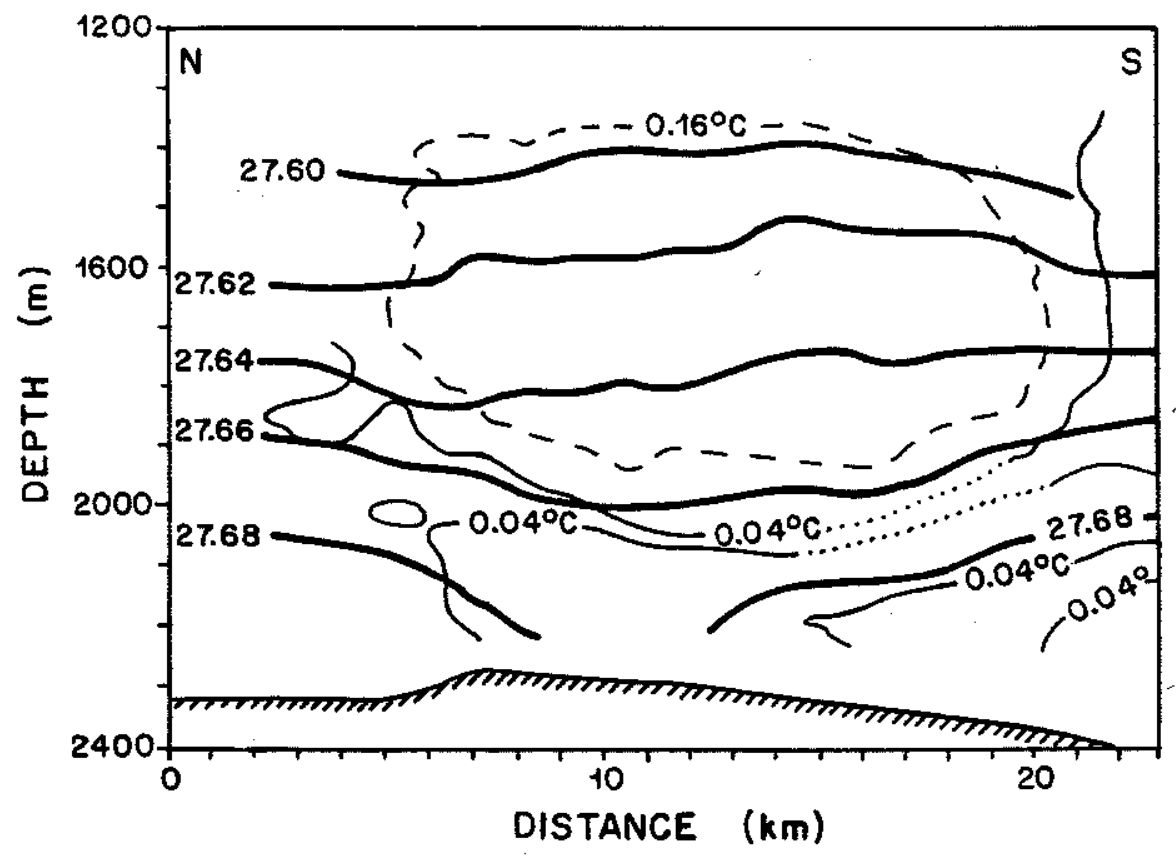

Figure 2.12: A north-south potential density section ( $\sigma_{\theta}$, heavy solid lines) at the Juan de Fuca Ridge near $45^{\circ} \mathrm{N}, 130^{\circ} \mathrm{W}$. Temperature anomaly (light solid and dashed lines) is defined by the difference from a background linear relation between potential density and potential temperature. The maximum anomaly near $1800 \mathrm{~m}$ depth is $0.28^{\circ} \mathrm{C}$. Adapted from Baker et al. (1987). 
designed mainly to provide helium measurements in the thermocline). These data were mapped onto several temperature surfaces spanning mid-depth. The data were highly variable, perhaps because of relatively poor vertical resolution in deep water, and no large-scale coherent feature was found.

No tracers of a hydrothermal influence have been observed thus far outside the rift valley of the Mid-Atlantic Ridge. Yet hydrothermal sources are believed to exist at more than 11 locations along the ridge between $26^{\circ} \mathrm{N}$ and $10^{\circ} \mathrm{N}$ based on measurements of manganese, methane, and helium within the rift valley (manganese by Klinkhammer et al., 1985; methane by Charlou et al., 1988; helium by Jenkins et al., 1980). These sources have been found at various levels in the water column, between $2500 \mathrm{~m}$ and $4000 \mathrm{~m}$ depth (see also Figure 2.15). This section of the ridge is also one in which the conductive heat source is relatively high, according to the geographic distribution shown by Langseth and Von Herzen (1971). Another such ridge section is near $45^{\circ} \mathrm{N}$ and the third is between $20^{\circ} \mathrm{S}$ and $35^{\circ} \mathrm{S}$.

In previous sections evidence has been presented for a cool, fresh influence on isopycnal surfaces and for the vertical spreading apart of isotherms near a hydrothermal heat source on the Mid-Atlantic Ridge. This result may be used as a guide to suggest that the expected large-scale signal from both hydrothermal and conductive heat sources along the crest of the Mid-Atlantic Ridge is relatively fresh and cool water spreading along a broad range of isopycnal surfaces. The range would depend on the bathymetry and locations of hydrothermal systems, for example, the degree of isolation of segments of the ridge crest from surrounding water. The strength of the signal depends on the strength and number of sources, and on the speed of the mean flow in the region. At the TAG site, the observed salinity anomaly from a background $\theta-S$ curve was about $.004 \%$ (section 2.3 ). If this anomaly were simply diluted over the $100 \mathrm{~km}^{2}$ area of the ridge segment the average anomaly would be small, about $.001 \%$, and hence unobservable far from the source. The buoyant plume, however, gives rise to a salinity flux past the equilibrium level which removes salt at the rate $2.5 \times 10^{3} \mathrm{~g} / \mathrm{s}$ (section 2.4.1). Then for 100 vents in a box $1000 \mathrm{~km}$ by $1000 \mathrm{~km}$ by $1 \mathrm{~km}$, an anomaly of $.005 \%$ could be generated in about $500 \mathrm{yrs}$. A mean current of $0.01 \mathrm{~cm} / \mathrm{s}$ could flush this box in a similar time, so the flow must be very weak or the number of vents must be greater for an observable signal. The conductive heat flux helps to increase the anomaly or allow a stronger current. In this section, observations are examined for evidence of these effects.

Other properties besides salinity with a significant vertical gradient would be expected to be mixed upwards by heating and thus be anomalous on a constant density surface. An estimate of the anomaly can be made by multiplying the mean vertical gradient by the mixing height, $z^{*}=300 \mathrm{~m}$. In the depth range $2500 \mathrm{~m}$ to $3500 \mathrm{~m}$ the mean oxygen and silica gradients are $-.03 \mathrm{ml} / 1 / \mathrm{km}$ and $-10 \mu \mathrm{mol} / 1 / \mathrm{km}$, 
giving anomalies of about $+.01 \mathrm{ml} / \mathrm{l}$ for oxygen and $+3 \mu \mathrm{mol} / 1$ for silica. This size oxygen anomaly is unobservable while the silica anomaly is marginally observable. At deeper levels the expected anomalies are somewhat greater. At depths below $4000 \mathrm{~m}$ (temperatures less than $2^{\circ} \mathrm{C}$ ), the mean gradients are $+.13 \mathrm{ml} / 1 / \mathrm{km}$ and $18 \mu \mathrm{mol} / 1 / \mathrm{km}$, giving anomalies of $-.04 \mathrm{ml} / 1$ for oxygen and $+5 \mu \mathrm{mol} / 1$ for silica. At these levels the expected oxygen anomaly is marginally observable.

One way to examine variations in the $\theta-S$ relation is to define a reference $\theta-S$ curve and calculate a salinity anomaly by subtracting the reference value of $S$ from the observed $S$ at a given $\theta$. A salinity anomaly thus defined will be used below as an indicator of variations in the $\theta-S$ relation. Another way is to plot salinity on isothermal surfaces as by Worthington and Wright (1970). In this study, plots of salinity on isopycnal surfaces are used since the influence of a source such as geothermal heating spreads by advection and diffusion primarily along a constant density surface. Most of the variation of salinity is expected to be caused by the mixing of salty deep water from the northern North Atlantic with fresh bottom water from the South Atlantic. The object is to see if the pattern of variation is consistent with a local vertical mixing effect around the ridge, which would be suggestive of heating. Mechanical vertical mixing by time-dependent vertical motion of water over the rough topography of the ridge could be important near topographically isolated valleys, but the speed required of deep flow $(50 \mathrm{~cm} / \mathrm{s}$, appendix A) to make this effect important elsewhere seems unrealistically high.

A quantity which may be related to heating is stability $\left(-\frac{\partial \rho}{\rho \partial z}\right.$, where $\rho$ is referenced to the in situ pressure). The distribution of stability and its relation to deep water masses in the Atlantic is discussed in appendix A. Although there can be a more complicated vertical structure in places, generally deep and bottom water are separated by a high stability layer in which density increases rapidly with depth. This is the basic state on which there may be variations related to local sources. The strong vertical mixing over scales of several hundred meters at the TAG site (section 2.3) suggests that layers affected by vent systems may have reduced stability. Stability is not a true tracer since there is no conservation equation for it; however, it is examined here because it is related to flow and mixing near a heat source.

There is a significant east-west variation in the $\theta-S$ relation at $13^{\circ} \mathrm{N}$ and $24^{\circ} \mathrm{N}$ (Figure 2.13). At these latitudes the $\theta-S$ relation between about $1.8^{\circ} \mathrm{C}$ and $2.5^{\circ} \mathrm{C}$ lies on the fresh side of the Worthington-Metcalf mean. Above $2.5^{\circ} \mathrm{C}$ at $24^{\circ} \mathrm{N}$ the water is saltier in the east because of the influence of high salinity Mediterranean water. Near $2.0^{\circ} \mathrm{C}$ or roughly $4000 \mathrm{~m}$ depth, the anomaly is about $-.015 \%$, calculated as the salinity difference at constant temperature. Its distribution can be more easily seen in sections at $13^{\circ} \mathrm{N}$ and $24^{\circ} \mathrm{N}$ (Figure 2.14 ). The anomaly extends 
more than $1000 \mathrm{~km}$ west of the crest and across the eastern trough at somewhat deeper levels.

The anomaly can also be calculated as the salinity difference at a constant density (Figure 2.15). The amplitude is larger because of the angle between isopycnals and isotherms in the $\theta-S$ plane. Also shown is a profile of stability at a station at $13^{\circ} \mathrm{N}$. The relation between the maximum negative anomaly and maximum stability indicates that the anomaly occurs at the transition from salty deep water of northern origin to fresh bottom water of southern origin. For stations above and east of the ridge, the anomaly is a result of the smoothing of the knee in the mean $\theta-S$ relation (Figure 2.13). These variations reflect the basic water mass distribution in this part of the North Atlantic. For an increasing contribution of water from the south at a given temperature, though, the anomaly ought to be greatest in the southern-most station, and disappear to the north. Instead, the anomaly is greatest at $13^{\circ} \mathrm{N}$ and $24^{\circ} \mathrm{N}$, and is weaker to the south or to the north (Figure 2.15). The pattern is hard to infer from a few stations; to look for variations which may result from heating it is helpful to examine the distribution of properties on a density surface. The problem is to distinguish large-scale water mass variations from anomalies related to heating.

Maps of salinity on the isopycnal surfaces $\sigma_{4}=45.85$ (units of $\mathrm{g} / \mathrm{cm}^{3}$ are dropped, as is standard) and $\sigma_{4}=45.90$ have been prepared (Figure 2.16). Also displayed on both surfaces are oxygen concentration and dissolved silica. The final map on the upper surface is of stability, while the final map on the lower surface is salinity from the TTO program. These densities correspond roughly to a potential temperature of $2.2^{\circ} \mathrm{C}$ and $1.9^{\circ} \mathrm{C}$, and to depths of $3500 \mathrm{~m}$ and $4200 \mathrm{~m}$ in the western trough. The data come from WHOI cruises and the GEOSECS and TTO programs (section 2.2). The total variation of salinity on these surfaces is small, about $.04 \%$, and it was necessary to make corrections owing to salinity differences in the standard sea water used to calibrate the salinometers, which in turn are used to calibrate the CTD profiles (Mantyla, 1986). The corrected data are presented.

The basic pattern of the salinity distribution on both surfaces is of relatively salty water in the north and west, and fresh water in the south and east. High salinity water extends south along the western boundary past the equator while near the ridge water is lower in salinity. This structure results from the southward flowing deep western boundary current and northward flowing bottom water above the western flank of the ridge (Warren, 1981). Bottom water enters the eastern trough principally through the Romanche Fracture Zone, but there is also some flow through the Vema Fracture Zone near $11^{\circ} \mathrm{N}$ (Warren, 1981; Vangriesheim, 1980). In the eastern trough the bottom water is expected to flow north above the eastern 

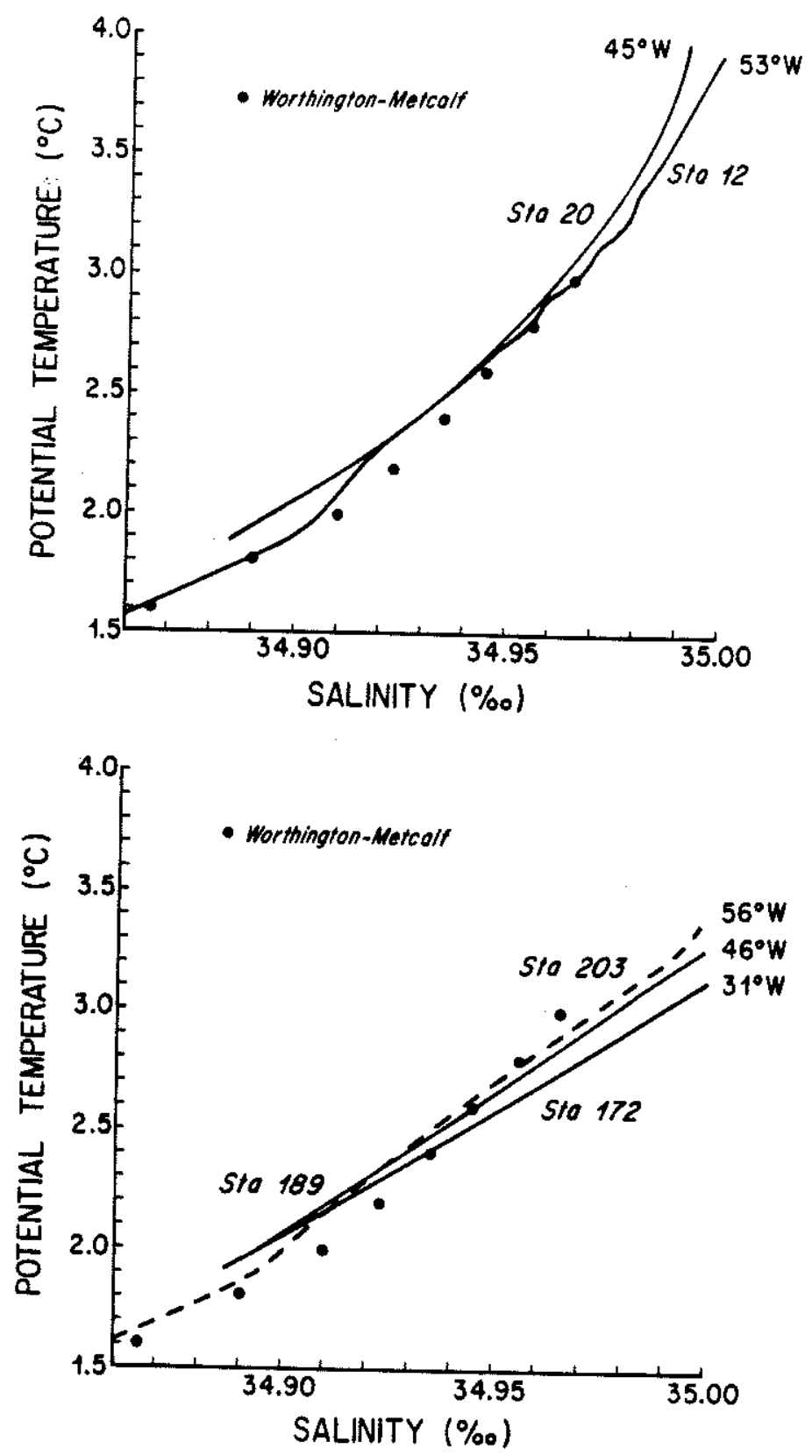

Figure 2.13: Potential temperature versus salinity for stations at $13^{\circ} \mathrm{N}$ (upper), and $24^{\circ} \mathrm{N}$ (lower) at several longitudes. At $13^{\circ} \mathrm{N}$ station 20 is near the crest while 12 is about $1000 \mathrm{~km}$ to the west. At $24^{\circ} \mathrm{N}$ a station east of the ridge is drawn for comparison (172). Station 189 is at the crest at $24^{\circ} \mathrm{N}$. Station 203 (dashed) is 

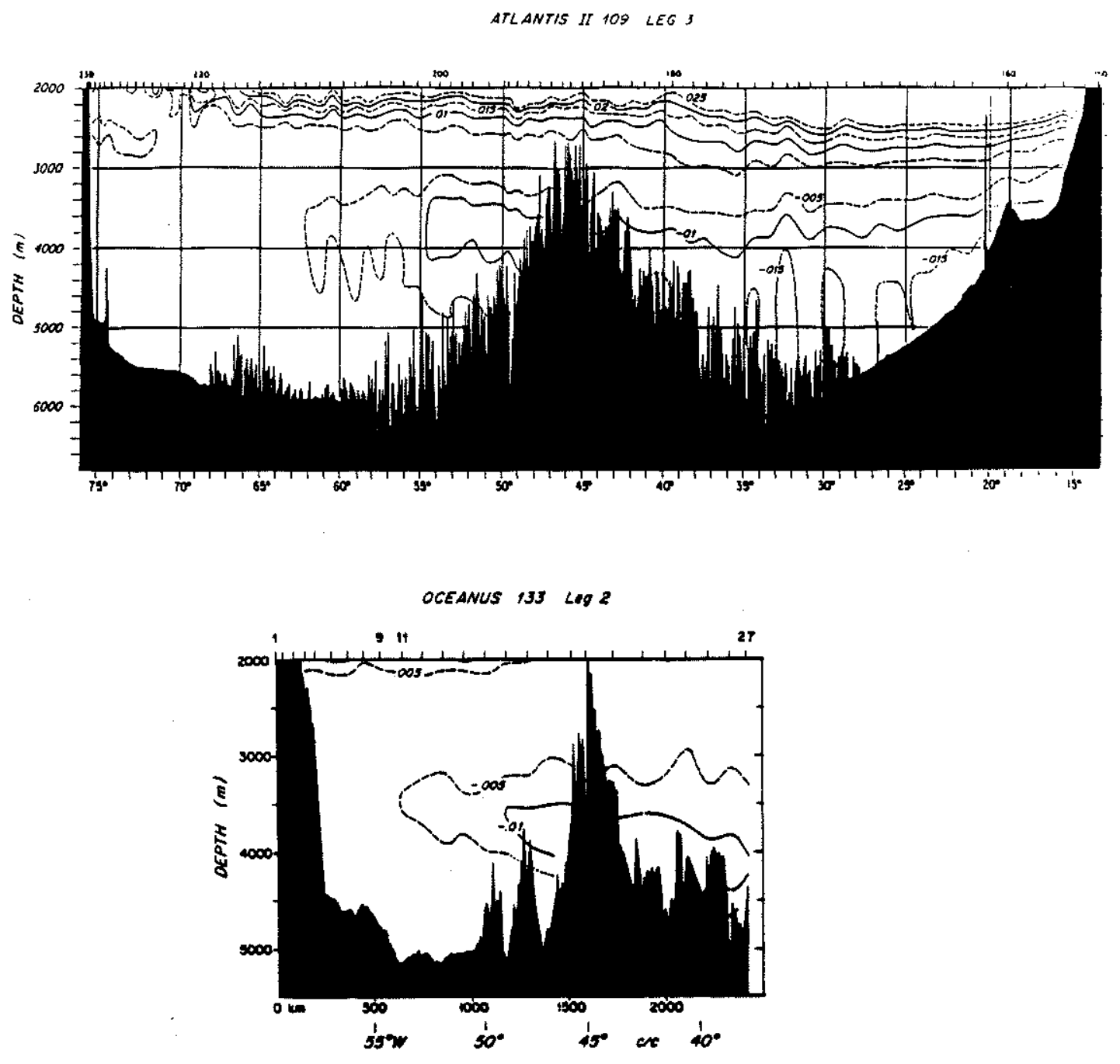

Figure 2.14: Maps of the salinity anomaly $(\%)$ relative to the mean deep water $\theta$-S curve of Worthington and Metcalf (1961) at $24^{\circ} \mathrm{N}$ (upper), and $13^{\circ} \mathrm{N}$ (lower). Negative values are fresh. Prepared by M. McCartney. 


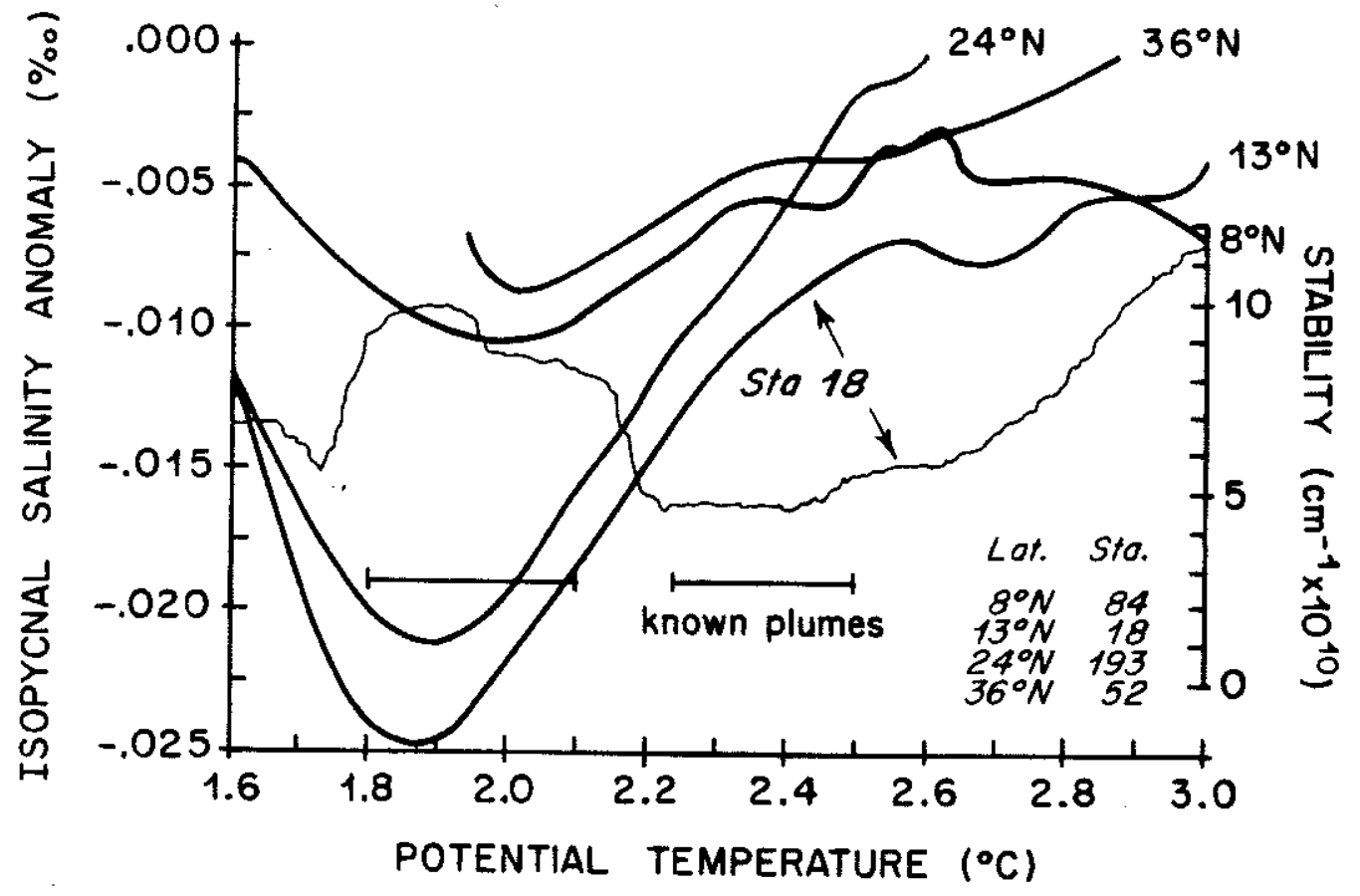

Figure 2.15: Salinity anomaly at several latitudes above the western flank of the ridge. The anomaly has been calculated along an isopycnal referenced to $4000 \mathrm{db}$ instead of along an isotherm to better represent its magnitude and temperature. Also shown is a stability profile (light solid line), and the temperature range of known hydrothermal vents above the crest. Vent locations from Klinkhammer et al. (1985); vent temperature data from Charlou et al. (1988), and P. Rona (data personally communicated). 
flank of the ridge roughly to $30^{\circ} \mathrm{N}$, according to the Stommel and Arons (1960) model. Thus fresh bottom water is expected to be flowing north on both sides of a limited portion of the ridge. On the upper surface low salinity is found extending northwest from the equator basically along the eastern side of the axis of the ridge. On both surfaces the low salinity along the western side is not continuous from south to north. Near $10^{\circ} \mathrm{N}$ salty water extends across the western trough at these densities. The basic pattern on these two surfaces, though, is consistent with the presence of boundary currents expected in the Stommel and Arons (1960) model.

On the upper surface in the latitude range roughly $10^{\circ} \mathrm{N}$ to $20^{\circ} \mathrm{N}$, there is a salinity minimum which is isolated from surrounding water by about a $.005 \%$ change in salinity. A minimum of similar amplitude also appears on the lower surface. In both cases it is defined by several stations from two different cruises. Furthermore where other ship tracks cross the $13^{\circ} \mathrm{N}$ section the salinities agree. Shifting one section or another by $.005 \%$ would not seem to be justified. Even so, the isolation has to be regarded as marginal. Station dependent salinometer errors could occur for unforseen reasons, or the distribution may reflect time-dependent flow, although the cruises are not widely separated in time (section 2.2). On the deeper surface the distribution of oxygen has the same basic shape as the salinity, with low oxygen correlated with low salinity (Figure 2.16). The oxygen distribution supports the isolation, but again the amplitude (from western trough to eastern trough) is marginal, about $.05 \mathrm{ml} / 1$. Finally, data from the TTO program also support the presence of a salinity minimum of about $.005 \%$ on the deeper surface. The TTO data alone on the upper surface (not shown) do not show a minimum; low salinity is connected to lower values east of the ridge. These data, however, are sparse compared to WHOI data in crucial places near the ridge and in the eastern trough. Thus they are not necessarily inconsistent with the WHOI data on the upper surface.

In contrast, on the upper surface oxygen is not extreme at the salinity minimum, nor is silica isolated (from west to east across the ridge) on either surface. Both of these properties are extreme at the eastern boundary. This should not be taken as contradictory evidence on the upper surface since the estimated magnitudes of a oxygen and silica anomaly indicated that neither would be likely to be observable. On the lower surface the absence of a silica maximum is not supportive of a heating effect, as water in the eastern trough where silica concentrations are high may flow into the western trough through some fracture zone.

The stability distribution on the upper surface shows an overall correlation with salinity such that stability is low where salinity is low (Figure 2.16). Exceptions to this occur near the South American and African continental margin and in the northern part of the eastern trough. The results of previous sections indicate that low salinity and low stability ought to be correlated where heat sources exist, but 
they do not apply to the overall distributions of either property. On a large scale stability is not necessarily correlated with any tracer from heat sources (Chapter 3). The overall distribution shown here probably reflects changes in the vertical shear of horizontal flow, since horizontal variations in stability on a given potential density surface are related to horizontal density variations on a level surface. The result here at least does not contradict the presence of heating at the ridge. Sections of stability which further illustrate its horizontal and vertical structure are discussed in appendix A.

\section{Alternatives to geothermal heating}

Mechanical mixing over a rough bottom is a conceivable but unlikely alternative. This requires unrealistically strong flow restricted to a limited part of the ridge. On the other hand significant vertical mixing probably does occur as water spills through fracture zones from one basin into another. Westward flow through fracture zones between $25^{\circ} \mathrm{N}$ and $12^{\circ} \mathrm{N}$ may contribute to produce the observed salinity minimum. Such flow is usually thought to supply bottom water to a basin which is more isolated from a deep water formation region; however, this cannot be the case here since any water from the eastern trough would be lighter than bottom water in the western trough.

\section{Conclusion}

The variations of salinity and oxygen shown by recent data resulting in extrema near the ridge on an isopycnal surface are close to the level of experimental error, but they are consistent between different data sets. It is therefore concluded that the observed salinity minimum is consistent with the geothermal heating of water as it moves north along a limited portion of the ridge.

These observations also demonstrate property distributions in basic agreement with deep boundary currents in the tropical Atlantic and suggest profitable areas for future measurements.

\section{Other regions}

In the South Atlantic Broecker and Takahashi (1980) describe some variations of the $\theta-S$ relation near $2.5^{\circ} \mathrm{C}$ using several GEOSECS stations in the western trough. They suggest that in the latitude range $4^{\circ} \mathrm{S}$ to $18^{\circ} \mathrm{S}$ the variation could be caused by a recent release of heat at the ridge crest, although the connection to the ridge is not established. The sections at $11^{\circ} \mathrm{S}$ and $23^{\circ} \mathrm{S}$ allow the connection to be checked (appendix A). The result is that the $\theta-S$ variations are correlated with oxygen variations and that they can only be caused by lateral movement, not vertical mixing. Decreasing salinity and oxygen to the south and east means that a weak flow near $2.5^{\circ} \mathrm{C}$ from the southern Angola Basin to the Brazil Basin could bring relatively fresh water and account for the observed oxygen and $\theta-S$ variations. The $\theta-S$ variations are strong enough to overwhelm any geothermal 
signal at slightly lower temperatures.

By comparison, in the South Pacific, where the large-scale influence of hydrothermal sources is traceable in part by the distributions of helium and manganese, there is a correspondence between these tracers, a $\theta-S$ anomaly, and low stability approximately in the range $5^{\circ} \mathrm{S}$ to $20^{\circ} \mathrm{S}$ (Lupton and Craig, 1981; Reid, 1982). Above the East Pacific Rise near $30^{\circ} \mathrm{S}$, where the mid-depth total flow is eastward based on manganese measurements (Klinkhammer and Hudson, 1986), the correspondence has not been demonstrated. Thus in the restricted latitude range $5^{\circ} \mathrm{S}$ to $20^{\circ} \mathrm{S}$ both passive and active tracers are correlated. Outside this range the correlation is unclear. In the presence of background flow, however, the large-scale correlation between active tracers (e.g. density perturbations) and passive tracers is not necessary (Chapter 3). A meridional section of stability at $180^{\circ} \mathrm{W}$ and a zonal section at $43^{\circ} \mathrm{S}$ have been presented by Reid (1986) without interpretation. In the $180^{\circ} \mathrm{W}$ section at latitudes near $25^{\circ} \mathrm{S}$, helium-3 and low stability coincide at about $2500 \mathrm{~m}$ depth. Further north on this section the cores or extreme values diverge by several hundred meters although there is still a general alignment. Unfortunately these sections are too far south and west of the tracer measurements to make a satisfactory comparison.

\subsection{Summary}

In this chapter the physical characteristics of seawater in an active convection region driven by geothermal heating are described, using a unique data set from the first such region to be found in the Atlantic. A cold, fresh plume spreading in a layer $300 \mathrm{~m}$ to $450 \mathrm{~m}$ above the bottom was discovered at $26^{\circ} \mathrm{N}$ on the Mid-Atlantic Ridge. An explanation is offered for the observed temperature and salinity characteristics which is corroborated by turbulent plume model calculations. The dynamical significance of the background temperature and salinity fields on plume characteristics is emphasized in the model calculations. For instance, the difference between the equilibrium and final height of the plume, which may influence the magnitude of the observed anomalies, is controlled by them. The $\theta-S$ characteristics of the plume could be predicted, and this kind of calculation could be useful in the future for predicting other chemical anomalies to test ideas about mixing. Fine-structure Cox number calculations support the idea that convection increases mixing over a relatively broad area of the rift valley.

To understand what happens past the stage at which the plume has reached its greatest height the effects of rotation must be taken into account. A general agreement between the turbulent plume model and observations motivates using the turbulent plume to drive the flow in the next larger-scale regime, generating a 
vortex and isopycnal deformation above the heat source. The effect of the buoyant plume on the surrounding environment is explored using a linear model for flow with rotation. In this regime, the upwelling water above the source is returned downward by diffusion over a broad area to close the circulation cell. Horizontal flow is generated by this vertical motion because of the presence of rotation. The results suggest that the vortex is of sufficient magnitude to trap water in a local recirculation. This could have a crucial effect on the expected dispersal of properties, including minerals, tracers, and organisms to other areas of the ridge and to the interior.

The results of the smaller-scale studies are used as a guide to possible large-scale effects of heating, such as a low salinity source. Over a limited part of the MidAtlantic Ridge roughly between $10^{\circ} \mathrm{N}$ and $20^{\circ} \mathrm{N}$, a salinity minimum on two density surfaces was found, of magnitude near the level of error in comparing different data sets. The presence of a minimum is supported by observations from several data sets. Although the interpretation of the minimum as an effect of heating is questionable because of its magnitude, alternative explanations seem less likely.

Future measurements in the Atlantic ought to be made in the region of the Mid-Atlantic Ridge, especially across the ridge between $26^{\circ} \mathrm{N}$ and the equator. A crucial section would be near $16^{\circ} \mathrm{N}$ to $18^{\circ} \mathrm{N}$, including some sampling in nearby fracture zones. For instance, the Kane Fracture Zone, just south of the $24^{\circ} \mathrm{N}$ section needs to be resolved with hydrographic stations on both flanks to see if the extreme property values can be traced to it. The possibility of westward flow through the Kane Fracture Zone has been mentioned previously, but the existing evidence was not thought to be of adequate coverage or accuracy to make a case for such flow (Warren, 1981). Observations should also be made at the Fifteen Twenty Fracture Zone for similar reasons. Finally, measurements should be designed to address the question of the horizontal flow forced by hydrothermal systems. Existing near-field observations are not broad enough to encompass it, while the basin-wide sections are too widely spaced to sample it.

The isopycnal deformation implied by the model with rotation can be extended along the ridge, by recognizing that the heat source also extends along the ridge. A representation of this deformation is used in the next chapter as the forcing for a study of the large-scale effects of the heating, including the variation of rotation with latitude. 


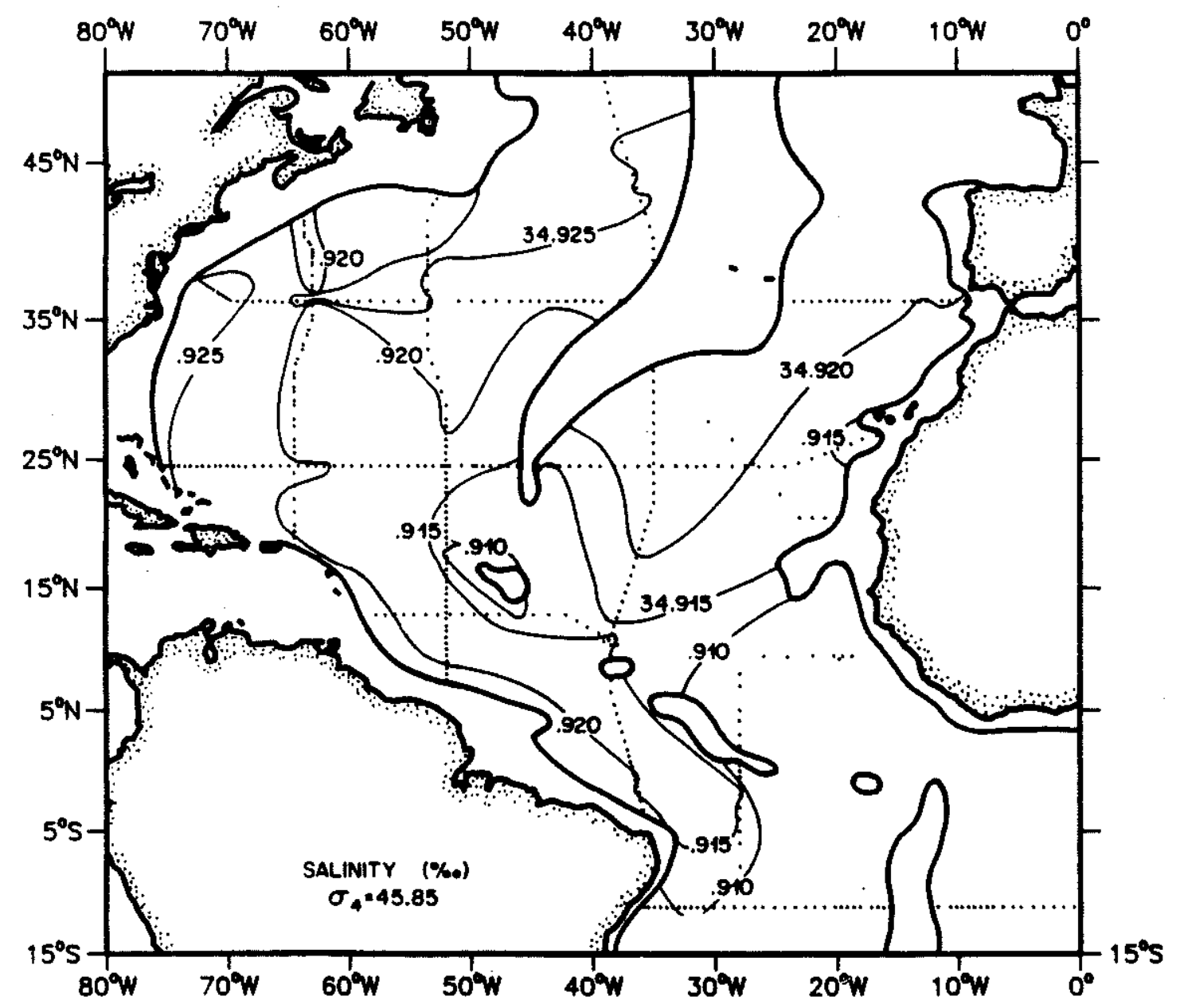

Figure 2.16: Salinity $(\%)$ on $\sigma_{4}=45.85$. Topography adapted from Worthington and Wright (1970). 


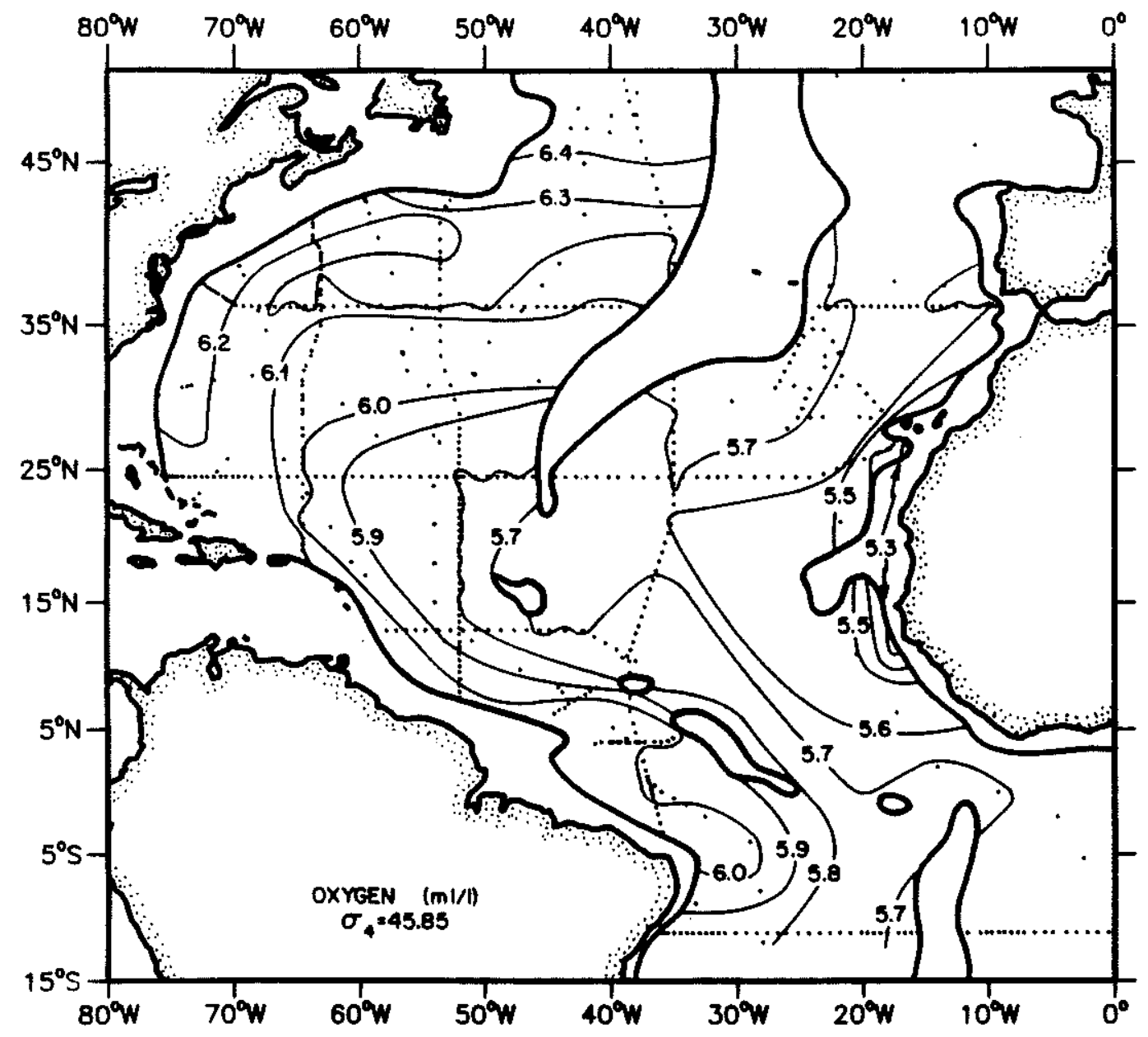

Figure 2.16: Oxygen (ml/1). $\sigma_{4}=45.85$. 


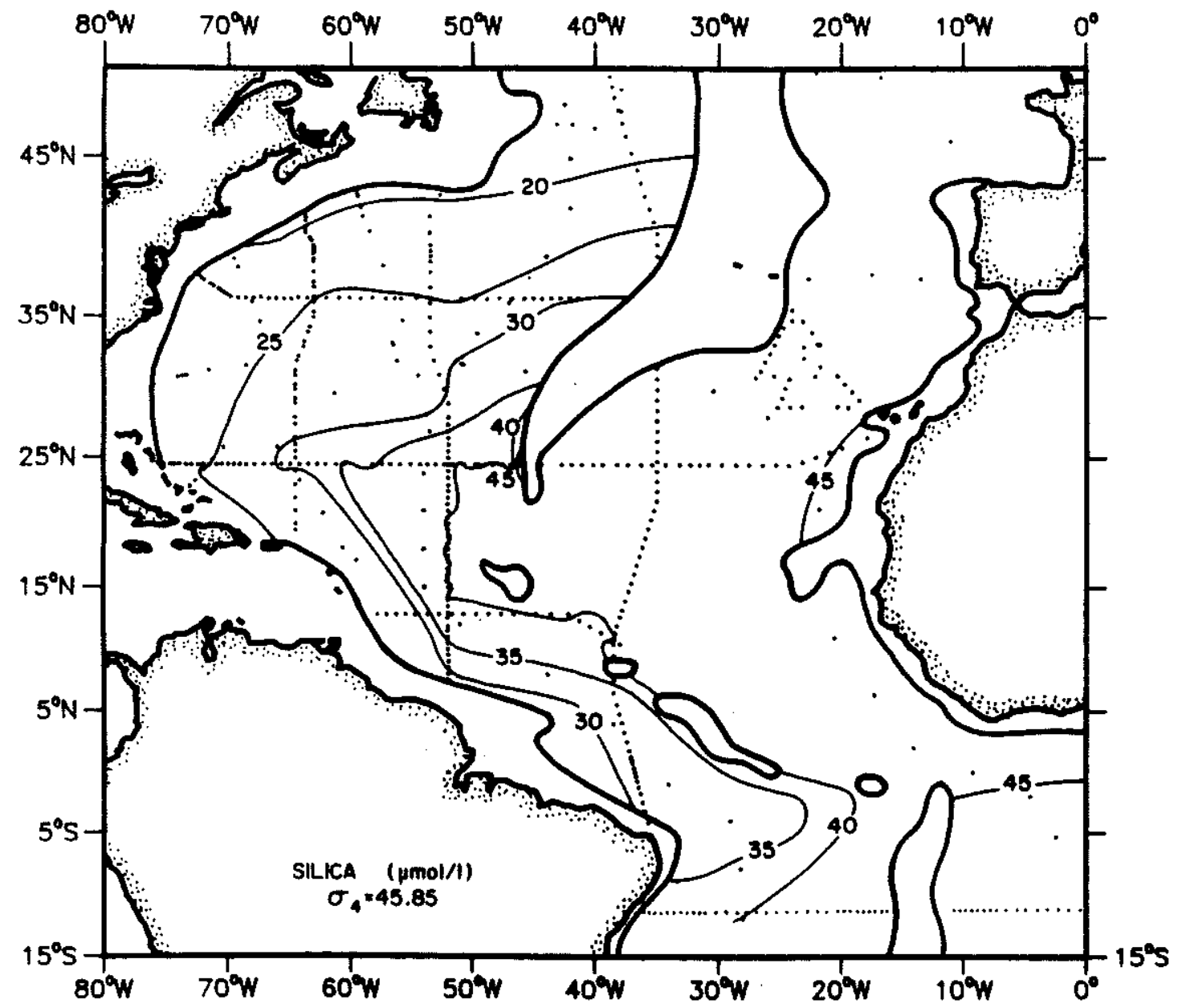

Figure 2.16: Silica $(\mu \mathrm{mol} / 1), \sigma_{4}=45.85$. 


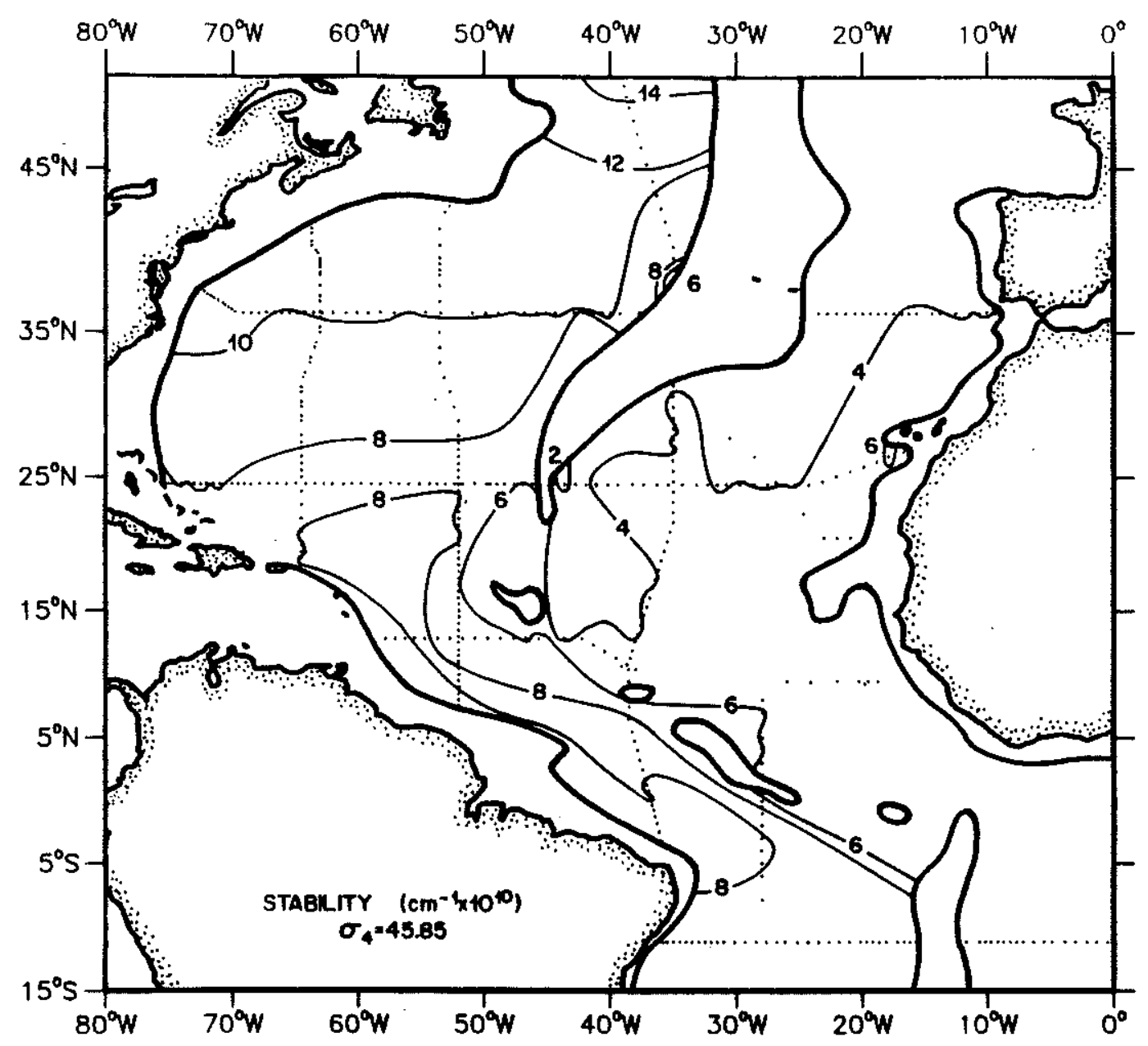

Figure 2.16: Stability $\left(\mathrm{cm}^{-1} * 10^{10}\right)$ on $\sigma_{4}=45.85$. 


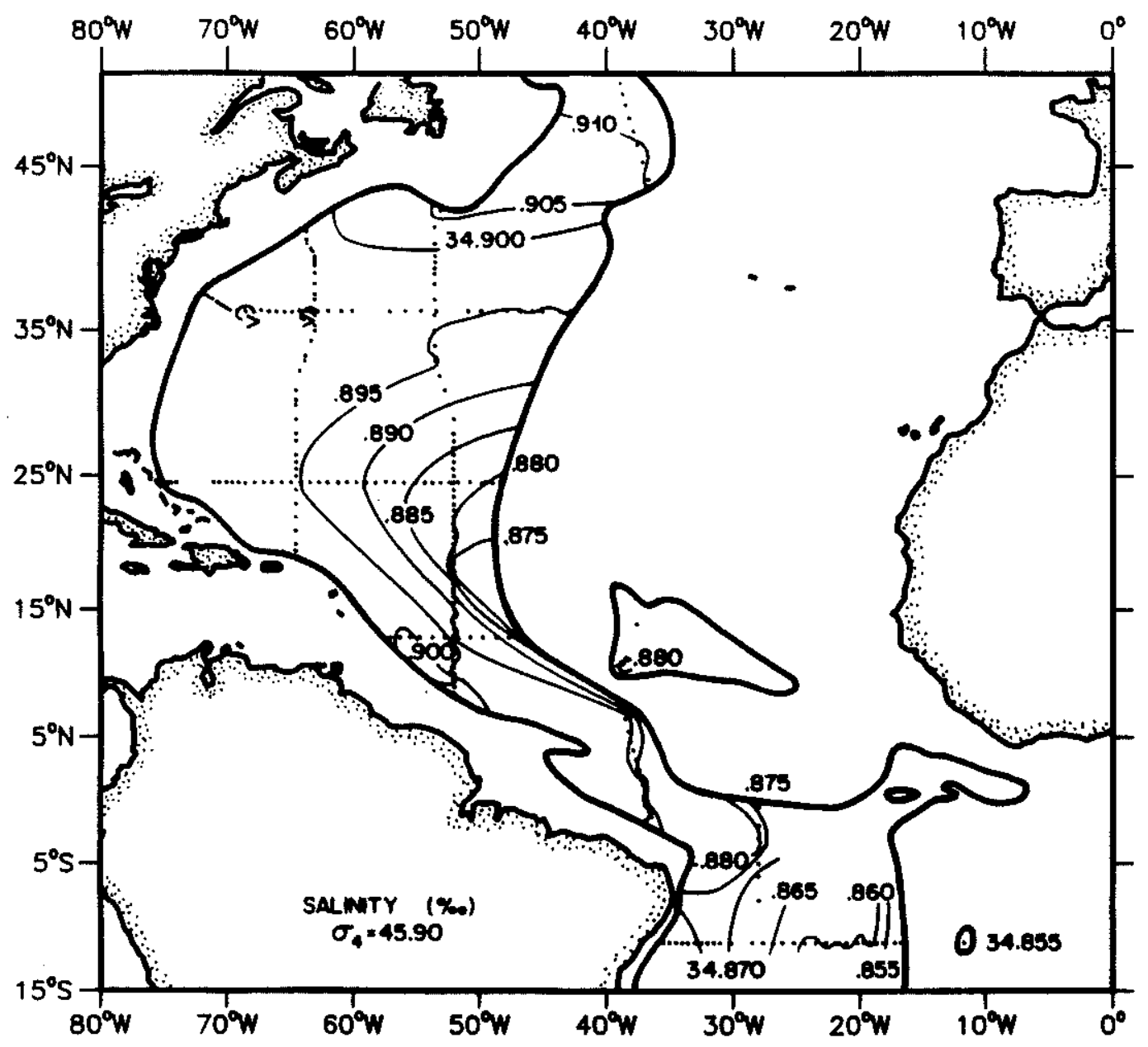

Figure 2.16: Salinity $(\%), \sigma_{4}=45.90$. 


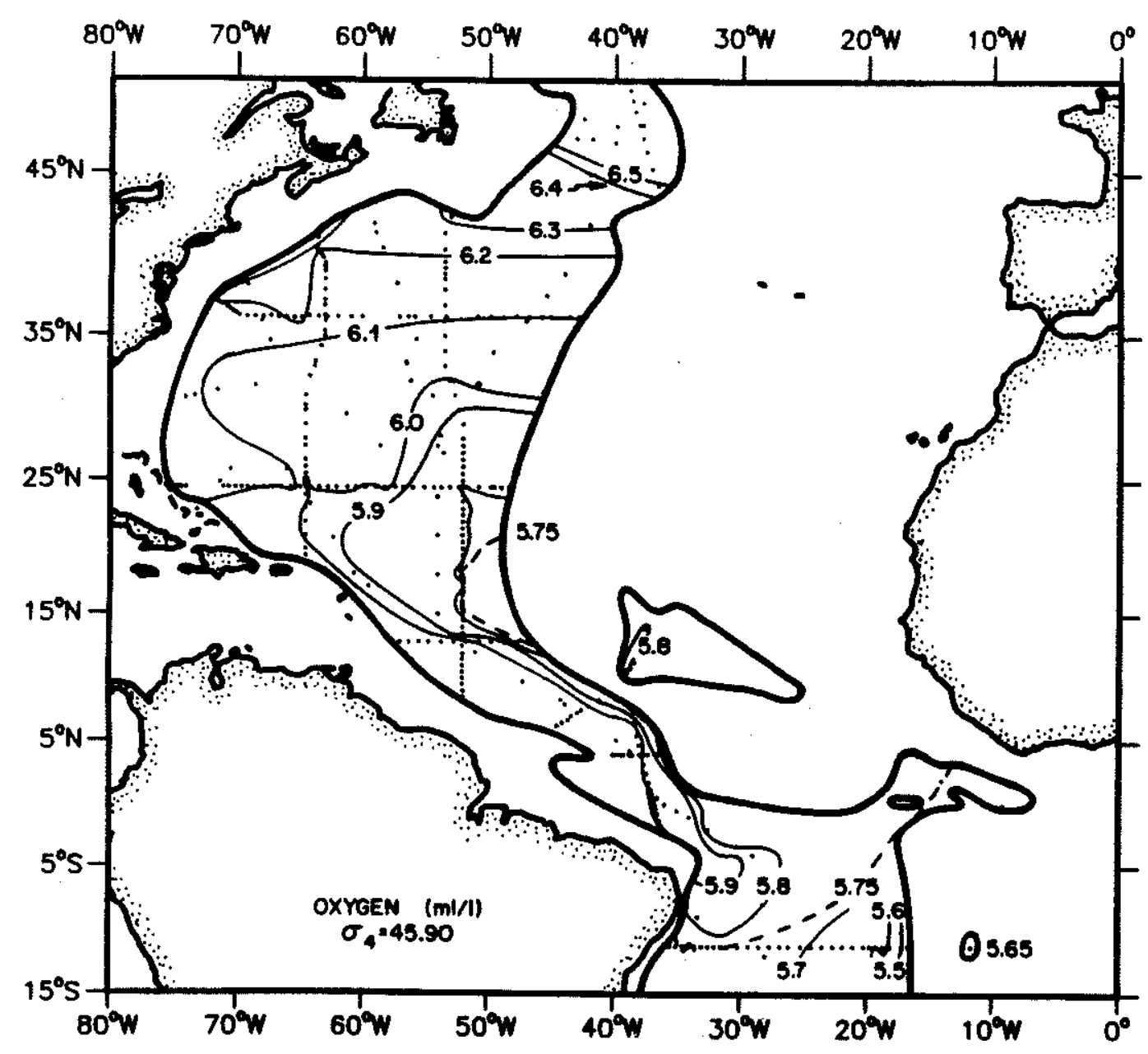

Figure 2.16: Oxygen $(\mathrm{ml} / 1)$ on $\sigma_{4}=45.90$. Values in the southeastern part of the eastern trough are from the Levitus Atlas. 


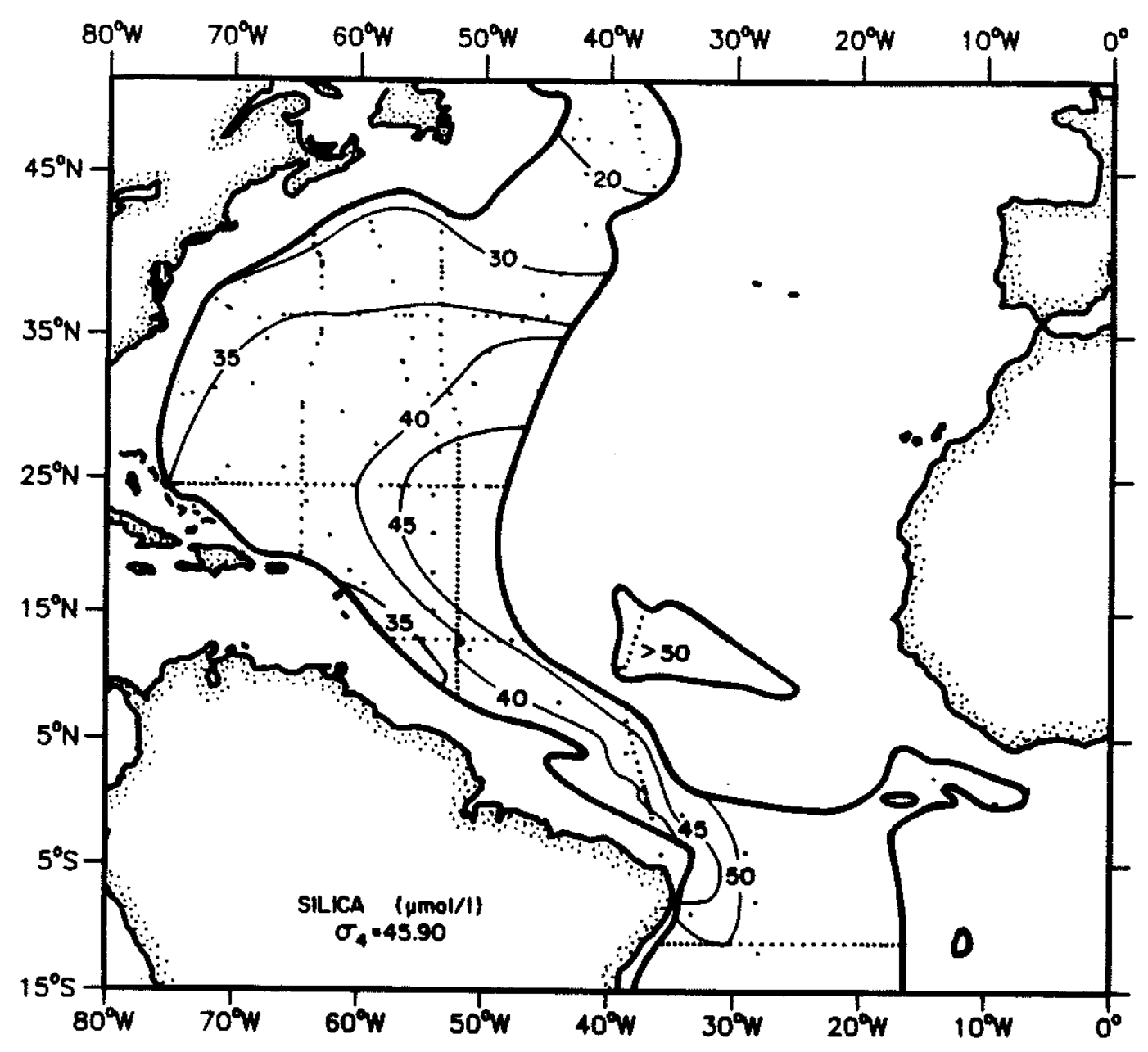

Figure 2.16: Silica $(\mu \mathrm{mol} / 1), \sigma_{4}=45.90$. 


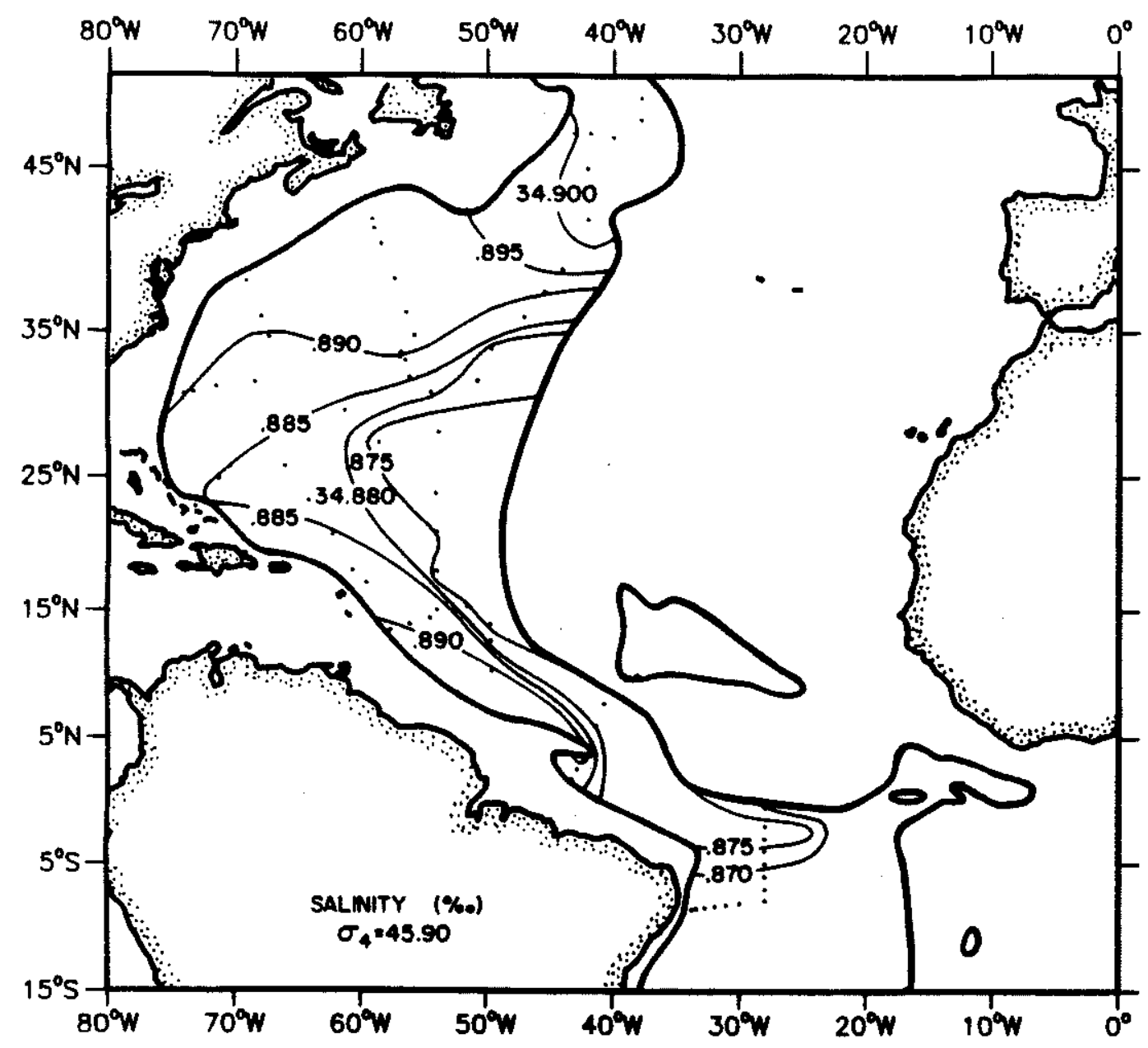

Figure 2.16: TTO salinity $(\%)$ on $\sigma_{4}=45.90$. 


\title{
Chapter 3
}

\section{The Large-scale Influence of a Heat Source}

\begin{abstract}
An approximate temperature perturbation equation for a continuously stratified deep ocean layer on a sphere is presented. A background flow is allowed, forced by uniform upwelling. Solutions with truncated vertical structure are given which are forced by a representation of geothermal heat sources. The solutions exhibit a range of behavior from passive advection to upstream influence, depending on the ratio of the background flow speed to long wave phase speed. Owing to the different phase speeds of the vertical modes, the forced structure is dispersed, which can result in both positive and negative temperature anomalies upstream and downstream of the source. This dispersal is enhanced at low latitudes. Depending on the strength of the imposed temperature anomaly at the source, there may or may not be a reversal of the flow field. Thus the correlations between temperature, geostrophic shear, and a passive tracer such as ${ }^{3} \mathrm{He}$ can be positive, negative, or zero away from the source. Some of these ideas are illustrated in an example calculation for the South Pacific, by comparing observed tracer and isopycnal depth distributions to a model solution. Extensions are made in which the importance of higher resolution and the effects of nonlinearity and background shear is examined.
\end{abstract}

\subsection{Introduction}

The existence of temperature-salinity anomalies and isopycnal deformations forced by geothermal sources in the rift valley, and of large-scale temperature-salinity anomalies, most extreme near the mid-ocean ridge, have been described in the previous chapter. These features provide the basic observational motivation for the study described here. In this chapter a model is presented for the large-scale effect of a heat source in a deep layer.

Geothermal sources introduce tracers of deep flow and have themselves a dynamical effect by supplying buoyancy at depth (Chapter 1). On a broad scale along 
certain segments of the mid-ocean ridge crest the magnitude of the heat-flux is estimated to be of order $1 \mathrm{~W} / \mathrm{m}^{2}$ (total hydrothermal plus conductive components, see chapter 1). In the immediate vicinity of a hydrothermal vent system, the average heat flux may be very large. Thus vertical motion is forced at many scales. Some of the details of flow near vents have been described in Chapter 2. The important dynamical concept about the near field for this chapter is the tendency for rotation to support circulating currents, allowing significant thermal anomalies to build up. Dynamical modes of the system are therefore excited and can distribute the energy introduced at the bottom elsewhere in the basin (section 3.2).

The direction of influence of the heating may be expected to depend partly on flow forced by other mechanisms, such as high latitude sinking. In the Stommel and Arons (1960) model, a uniform interior upwelling exists to balance this mass source. The upwelling forces a poleward and eastward interior flow. In a stratified ocean the upwelling constrains only the vertically integrated flow, and thus we do not know what to expect at any particular depth. However, the model has been found to explain consistently the existence of boundary currents and the largescale distribution of properties in different circumstances (Warren, 1981). Stommel (1982) sketched the westward influence of a mass source at a mid-ocean ridge in a basic state of no motion. But it is not obvious that there is westward influence in the presence of opposing flow, for instance that of the Stommel and Arons model. By including this background flow there is some hope of comparison with real property distributions, and thus it may be possible to assess the importance of forcing at a mid-ocean ridge.

Knox (1971) has examined the influence of a heat source in a temperature stratified fluid, with a goal of contrasting dynamically important fields such as temperature with the pattern that passive tracers might take. He examined the case of a heated horizontal strip of fixed vertical scale in an infinitely deep layer. His study was similar to this one in that he first looked at linear solutions and then nonlinear corrections, and that he used Fourier techniques. However his model was nonrotating, and thus did not include the possibility of an active westward influence caused by variations in earth's rotation.

Deep interior flow has received less attention than flow near boundaries, because it is weaker and harder to measure. It is crucial to determine the flow in the interior because the interior absorbs the water transported in boundary currents and somehow helps return it to the source. Recently observations have been made which indicate both westward and eastward flow in the tropical South Pacific at mid-depth (Lupton and Craig, 1981; Klinkhammer and Hudson, 1986; mid-depth is meant to denote water between about $1500 \mathrm{~m}$ and $3500 \mathrm{~m}$ depth). Such flow is contrary to the simplest expectation from the Stommel and Arons model. Furthermore no 
eastward flow or influence is present in Stommel's (1982) outline of the influence of a geothermal source. The aim of this study is to understand some competing mechanisms responsible for deep flow. The rich combination of tracers and dynamics in the geothermal heating problem is attractive and motivating.

Some of the basic aspects of the model have been presented elsewhere (Joyce and Speer, 1987). The main difference in this derivation is that the model here uses spherical coordinates instead of cartesian coordinates. Then the variation of earth's rotation is correctly represented, which has a significant effect on long wave phase speeds. After the model derivation, several examples, an application, and extensions are given which were not presented earlier. The extensions help one to understand the effect of heating in less idealized conditions.

\subsection{Standard Model}

The model domain is a region of lateral dimension $a=$ earth's radius, and thickness $\mathrm{H}=4 \mathrm{~km}$, meant to represent an abyssal layer below the thermocline with a flat bottom. Spherical coordinates are used with components $(\phi, \theta, z)$ where $\phi$ is longitude, $\theta$ is latitude (both in radians) and $z$ is height above the bottom, with velocity components $\mathbf{u}=(u, v, w)$. The basic stratification is assumed to be established by processes unrelated to geothermal heating and is specified. For convenience it is a linear function of the vertical coordinate.

The steady-state large-scale equations of motion for a thin sheet of fluid on a sphere are:

$$
\begin{gathered}
-2 \Omega \sin (\theta) v=\frac{-1}{\bar{\rho} a \cos (\theta)} p_{\phi} \\
2 \Omega \sin (\theta) u=\frac{1}{\bar{\rho} a} p_{\theta} \\
0=-p_{z}-\rho g \\
\frac{u}{a \cos (\theta)} \rho_{\phi}+\frac{v}{a} \rho_{\theta}+w \rho_{z}=K_{v} \rho_{z z} \\
\frac{1}{a \cos (\theta)} u_{\phi}+\frac{1}{a \cos (\theta)}(v \cos (\theta))_{\theta}+w_{z}=0
\end{gathered}
$$

where $\mathrm{p}$ is pressure and $\rho$ is density. The neglect of horizontal diffusion is the essential simplification which allows the characteristic method to be used below, and is expected to be reasonable away from boundaries in the interior. It is strictly smaller then vertical diffusion only if the horizontal diffusion $K_{H} \ll K_{v} L^{2} / H^{2}$, where $L$ is the lateral scale of variations in the density field in the interior. The vorticity equation is

$$
v=a \tan (\theta) w_{z}
$$


Taking $\partial / \partial z$ of this equation gives the relation between vertical velocity and zonal density variations,

$$
w_{z z}=\frac{-g}{\bar{\rho} a^{2}} \frac{\rho_{\phi}}{2 \Omega \sin ^{2}(\theta)} .
$$

The basic state is separated in the density and velocity fields

$$
\begin{gathered}
\rho_{\text {total }}=\rho_{o}(1+\gamma \bar{\rho}(z)+\epsilon \rho(\phi, \theta, z)) \\
\mathbf{u}_{\text {total }}=\overline{\mathbf{u}}(\phi, \theta)+\mathbf{u}^{\prime}(\phi, \theta, z)
\end{gathered}
$$

where $\bar{\rho}$ is the basic stratification, $\gamma=0\left(10^{-4}\right)$ and $\epsilon=0\left(10^{-5}\right)$ represent the relative strengths of the basic stratification and the perturbations forced by heating. The relative strength of the velocities is

$$
\frac{U^{\prime}}{\bar{U}}=\frac{g \epsilon H}{2 \Omega L \bar{U}}=C \frac{a}{L} \frac{\epsilon}{\gamma}, \quad C=\frac{g \gamma H^{2}}{2 \Omega a w_{o}}=\frac{c_{o}}{\bar{U}}
$$

where $\mathrm{w}_{o}$ is the upwelling velocity at the top of the layer and $c_{o}=\frac{g \gamma H}{2 \Omega a}$ is the phase speed of planetary waves. The horizontal derivatives are scaled by $\mathrm{L}^{-1}$ rather than the larger basin scale $a$. Substituting these forms into the density equation and subtracting the basic state balance $\bar{w} \bar{\rho}_{z}=K_{v} \bar{\rho}_{z z}$ gives

$$
\left(\overline{\mathbf{u}}+C \frac{a}{L} \frac{\epsilon}{\gamma} \mathbf{u}^{\prime}\right) \nabla \rho+\frac{L}{a} \bar{w} \rho_{z}+C w^{\prime} \bar{\rho}_{z}+C \frac{\epsilon}{\gamma} w^{\prime} \rho_{z}=\frac{L}{a P_{e}} \rho_{z z}
$$

where $\mathrm{P}_{e}=w_{o} H / K_{v}$. Two limits are useful to look at. In the limit $\mathrm{C} \rightarrow 0$ the equation becomes

$$
\overline{\mathbf{u}} \cdot \nabla \rho=P_{e}^{-1} \rho_{z z}
$$

which is passive advection in all three directions. In the limit $\mathrm{C} \rightarrow \infty$ the equation becomes

$$
\frac{\epsilon}{\gamma} \mathbf{u}^{\prime} \cdot \nabla \rho+w^{\prime} \bar{\rho}_{z}=\frac{\epsilon}{\gamma P_{e}^{\prime}} \rho_{z z}
$$

where $P_{e}^{\prime}=U^{\prime} H^{2} / a K_{v}$. In this equation the linear terms are the balance in Stommel's (1982) " $\beta$-plume", so this equation describes a nonlinear $\beta$-plume (Chapter 4).

Equation 3.9 is linearized for small $\epsilon / \gamma=L U^{\prime} / a c_{o}$, which means that the particle velocity is small compared to the long-wave phase speed. In addition, for $L<a$ vertical advection by the mean flow can be neglected, that is, parcels do not leave out the top of the layer too quickly. This gives 


$$
\overline{\mathbf{u}} \cdot \nabla \rho+C w^{\prime} \bar{\rho}_{z}=\frac{L}{a P_{e}} \rho_{z z} .
$$

Note that although the lateral scale of variation is smaller than the basin scale it is still much greater than the deformation radius, which is $\mathrm{O}(10 \mathrm{~km})$ in deep water. Taking $\partial^{2} / \partial z^{2}$ of the above equation and using $\rho_{z} / H \rho_{z z}<<1$, that is, that the vertical scale of the perturbation is small compared to the layer thickness, gives

$$
\overline{\mathbf{u}} \cdot \nabla \rho_{z z}+C w_{z z}^{\prime} \bar{\rho}_{z}=\frac{L}{a P_{e}} \rho_{z z z z} .
$$

The boundary conditions are $w^{\prime}=0$ at the top and bottom, and heat flux convergence $\rho_{z z}=0$ at the top and bottom. The upper boundary condition on $w^{\prime}$ is somewhat arbitrary, but is motivated by the desire to prevent the perturbations from affecting the upper layer. A different condition such as $w_{z}^{\prime}=0$ at the top would change the modal structure (see below) representing the solutions. This would not change the linear behavior in any important way, but it could change the nonlinear behavior. Ideally, no flux boundary conditions $\left(\rho_{z}=0\right)$ would be used because then the heat introduced at the source is redistributed in the basin without leaking out the boundaries. The $\rho_{z z}=0$ boundary condition on the density is used rather than a flux condition to allow the solution to be made up of components with simple sinusoidal vertical structure. In general with interior perturbations and a no flux boundary condition the heating $\rho_{z z}$ would be nonzero and require horizontal advection at the top and bottom to balance it. The horizontal density gradients implied by advection would give rise to vertical shear near the boundaries. In this model such turning of the velocity near the boundaries is not taken into account. The justification for this approach is its relative simplicity while still retaining some interesting physics.

The variables are expanded in the form

$$
\begin{gathered}
\rho=\sum_{m} \rho^{m} \sin (m \pi z) \\
w^{\prime}=\sum_{m} \frac{\rho_{\phi}^{m}}{m^{2} \sin ^{2}(\theta)} \sin (m \pi z) \\
u^{\prime}=\sum_{m} \frac{-\rho_{\theta}^{m}}{m \sin (\theta)} \cos (m \pi z) \\
v^{\prime}=\sum_{m} \frac{\rho_{\phi}^{m}}{m \sin (\theta)} \cos (m \pi z) \\
p=\sum_{m} \frac{\rho^{m}}{m} \cos (m \pi z)
\end{gathered}
$$


to satisfy the boundary conditions at the top and bottom. To illustrate the conditions under which individual terms in this expansion can be thought of as modes, consider representing density as $\rho=\hat{\rho}(\phi, \theta) G(z)$. Substituting into 3.13 and using 3.6 gives

or

$$
\overline{\mathbf{u}} \cdot \nabla \hat{\rho} G^{\prime \prime}+C \frac{-g \bar{\rho}_{z}}{\bar{\rho} a^{2} 2 \Omega \sin ^{2}(\theta)} \hat{\rho}_{\phi} G=\frac{L}{a P_{e}} \hat{\rho} G^{\prime \prime \prime \prime},
$$

$$
\frac{\overline{\mathbf{u}} \cdot \nabla \hat{\rho}}{\hat{\rho}_{\phi} C(\theta)}-\frac{g \bar{\rho}_{z} G}{\bar{\rho} G^{\prime \prime}}=\frac{L}{a P_{e}} \frac{\hat{\rho}}{\hat{\rho}_{\phi} C(\theta)} \frac{G^{\prime \prime \prime \prime}}{G^{\prime \prime}}
$$

where $C(\theta)=C / a^{2} 2 \Omega \sin ^{2}(\theta)$. The first term on the left is a function of $\phi, \theta$ only, the second of $z$ only, but the term on the right is mixed. However, if $\bar{\rho}_{z}$ is constant then $G$ is sinusoidal and the right-hand side of the equation is separated. Requiring $\bar{\rho}_{z}$ to be constant allows the equation to be separated with vertical diffusion, and the terms in the above expansion to be interpreted as dynamical modes.

Substituting these forms and also the Stommel and Arons velocity field scaled by $U, \mathbf{\mathbf { u }}=(-2 \phi \cos (\theta), \tan (\theta))$, gives finally the nondimensional equation

$$
\left[-2 \phi-\frac{C}{m^{2} \pi^{2} \sin ^{2}(\theta)}\right] \rho_{\phi}^{m}+\tan (\theta) \rho_{\theta}^{m}=-P_{e}^{-1} m^{2} \pi^{2} \rho^{m}
$$

The two parameters controlling the nature of the solution are the Peclet number $\mathrm{P}_{e}$ and $\mathrm{C}$, the ratio of the long-wave phase speed to the background flow strength. Typical values of these parameters are $\mathrm{P}_{e}=10$ and $\mathrm{C}=1$.

This equation is a first order partial differential equation already in characteristic form. It is solved by specifying an initial condition at a source and integrating along characteristics. The initial amplitude decays away from the source with the scale $P_{e} / m^{2}$. Thus high modes are trapped near the source. For this reason the solution is truncated to 3 modes; the importance of higher modes is discussed in a later section.

At the source, the amplitude of each mode is set by a projection of the given source vertical structure. Several example structures are chosen in the following to illustrate the dependence on source vertical structure. The amplitude scale of the perturbations is set by $\epsilon$, and for $\epsilon=10^{-5}$ the corresponding temperature perturbation $\left(\frac{\epsilon}{\alpha}\right.$, where $\alpha$ is the thermal expansion coefficient $\left.\left(2 \times 10^{-4 \circ} \mathrm{C}^{-1}\right)\right)$ is about $0.05^{\circ} \mathrm{C}$, which is typical of observed values (Reid, 1982). In the following solutions, potential temperature has been substituted for density according to $\rho=-\rho_{o} \alpha \Theta$.

\subsection{Solutions}

To illustrate the pattern of influence for different values of $\mathrm{C}$, two solutions are shown (Figures 3.1 and 3.2). The source extends from about $\phi=0.1$ to $\phi=0.4$ 
$\left(5^{\circ} \mathrm{N}\right.$ to $\left.25^{\circ} \mathrm{N}\right), 1500 \mathrm{~km}$ from the eastern boundary. From these low latitudes the source can potentially influence almost any other point in the basin. The vertical structure of the source is a positive temperature perturbation symmetric about middepth. Only odd mode numbers have a nonzero projection on this vertical structure and their amplitudes are $\theta^{1}=0.61, \theta^{3}=-0.36$, and $\theta^{5}=0.2$. The Peclet number is 100 , so diffusion is weak.

The influence is mostly north and east, basically downstream. Mode 1 initially penetrates to the west somewhat but then turns back. Mode 1 dominates the temperature pattern, but the negative amplitudes from mode 3 are evident east of mode 1 in the lowest panel of the middle colulmn. At mid-depth the temperature source appears to spread eastward as well as westward before bring carried north in the background flow, owing to mode separation. Note that a passive tracer such as helium would appear only along the eastern lobe, directly downstream of the source. Since the temperature deviation represents an isopycnal deviation, an upstream influence of temperature is equivalent to an upstream influence of vertical shear in the velocity field. Thus a passive tracer such as helium need not be correlated with geostrophic shear forced by the source.

The total pressure is slightly twisted by the perturbations, as measured by the size of $C a / L \epsilon / \gamma$, in this case 0.1 . The sense of the modification is given by the mode 1 velocity which is clockwise (anticylonic) in the upper half of the layer and counterclockwise (cyclonic) in the lower half. At $z=2000 \mathrm{~m}$ height the perturbation pressure is zero.

The mode separation is emphasized by increasing $\mathrm{C}$ to 1 (Figure 3.2). The strong dependence of the solution on mode number and latitude is clear. Now mode 1 penetrates almost due west, while modes 2 and 3 turn north and east within $1000 \mathrm{~km}$ to $2000 \mathrm{~km}$ of the source. At $z=2000 \mathrm{~m}$ the decay west of the source is entirely a result of separation rather than diffusion. The separation is especially evident in the temperature field shown in the lower middle panel in which the temperature perturbation increases west of the source and becomes negative north of the source. At this level mode 3 has a negative amplitude and thus induces the westward increase by separation. Also evident is the stronger decay of the higher mode to the north and east.

Owing to the reduced strength of background flow relative to perturbation flow the low latitude pressure field is dominated by the mode 1 plume. On the equatorward side of the plume the flow is westward in the upper half and eastward in the lower half of the layer.

The final example illustrates the situation when the source extends from the equator to the northern boundary. (Figure 3.3) Now for $\mathrm{C}=1$ the characteristics fill out a larger area of the basin. The vertical structure of the source is different 

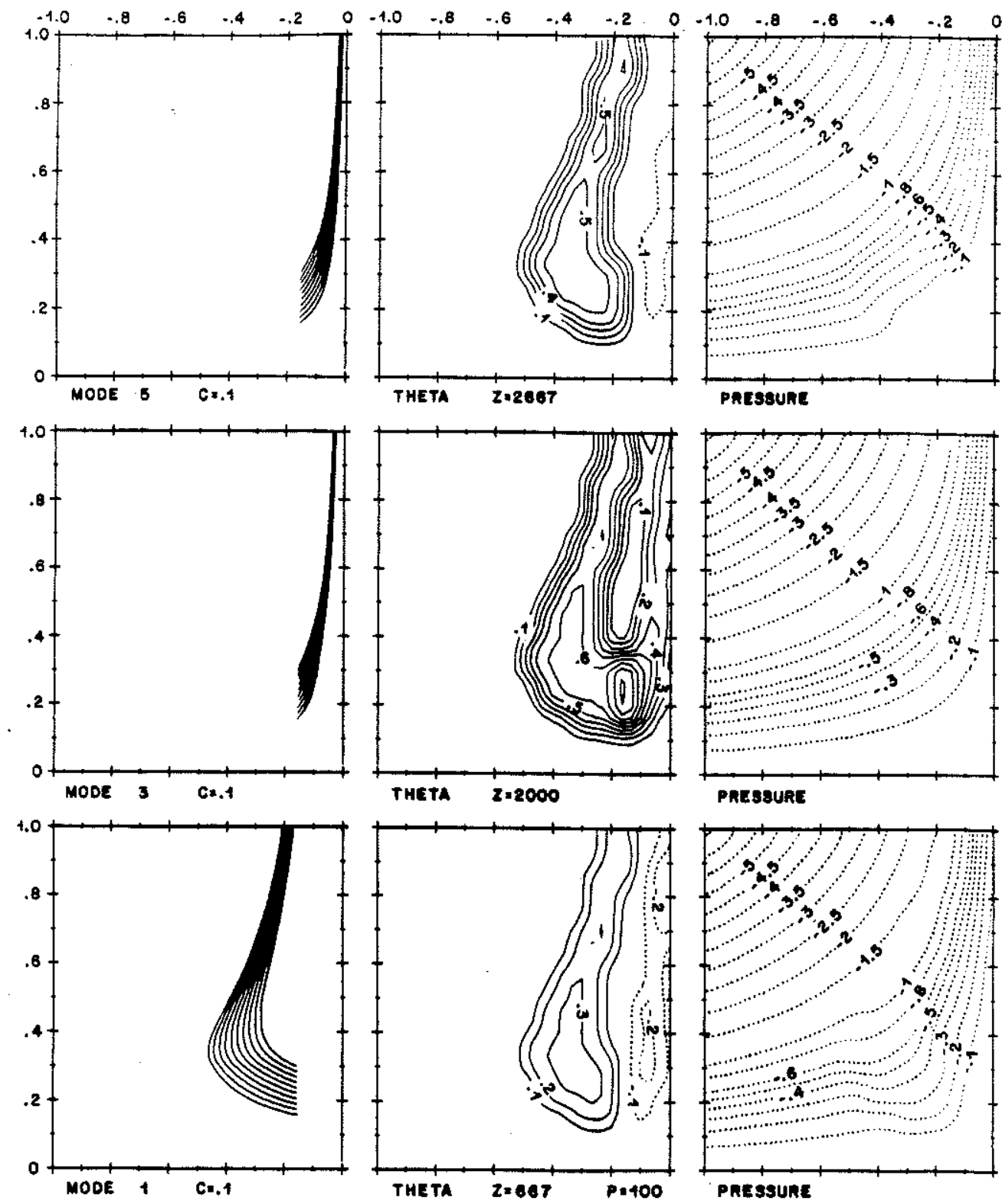

Figure 3.1: a) Solution for a mid-depth positive temperature anomaly, the axes are longitude and latitude in radians. In the left-most column the characteristics for mode 1,3 and 5 are shown (only odd modes are forced). The middle and right columns show perturbation temperature and total pressure at 3 different levels, $z$ $=667 \mathrm{~m}, \mathrm{z}=2000 \mathrm{~m}$, and $\mathrm{z}=2667 \mathrm{~m}$ height above bottom. $\mathrm{C}=0.1, \mathrm{Pe}=100$, $\mathrm{C} a \epsilon / L \gamma=0.1$. 

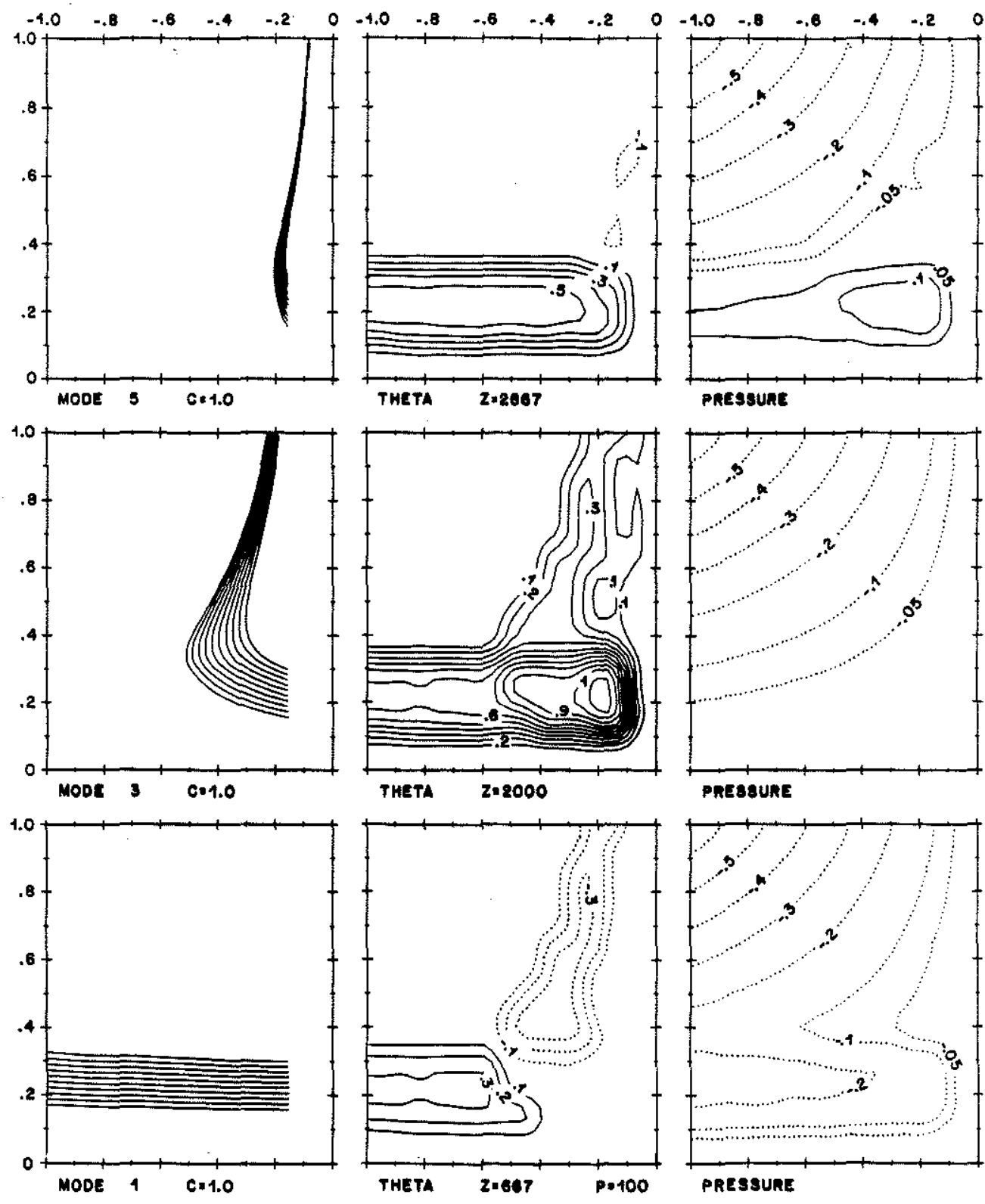

Figure 3.2: b) Solution for $\mathrm{C}=1, \mathrm{C} a \epsilon / L \gamma=1$. 

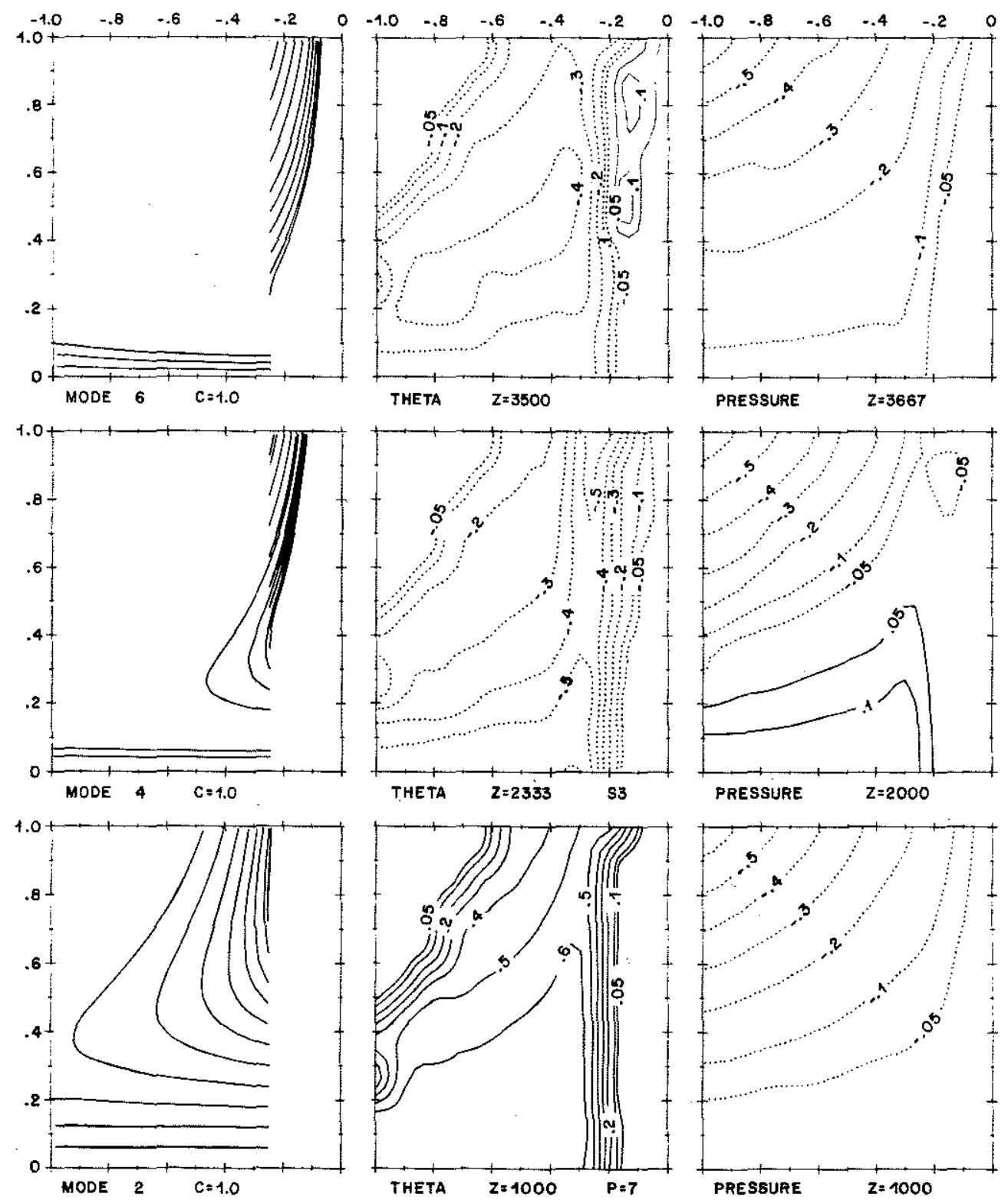

Figure 3.3: Solution for a mid-depth stability minimum, $\mathrm{C}=1, \mathrm{Pe}=7$. Only even modes are forced. The source extends from the equator to the northern boundary at $60^{\circ} \mathrm{N}$.

and represents a stability minimum at mid-depth, so that isotherms are forced apart. The mode amplitudes are $\theta^{2}=0.69, \theta^{4}=-0.24$, and $\theta^{6}=0.076$. This structure is antisymmetric so only even modes are non-zero. The main difference is that now the perturbation flow runs into the equator, as well as the other boundaries, where it must turn to close the circulation. Stronger diffusion damps the mode amplitudes slightly more than before.

These solutions are meant to illustrate the modal separation and the influence it has on the solution. The separation arises because of the presence of background flow, which disperses the purely westward-pointing $\beta$-plume. Thus characteristics which would otherwise run straight from the source to the western boundary, may, 
with some small background flow, spread into very different regions of the basin. The other key characteristic of the solutions is the tendency for small vertical scales to be passively advected and damped. These concepts are independent of the choice of vertical structure above the source, although the details of the solution at particular levels depend strongly on the initial mode amplitudes. Both separation and diffusion together give rise to temperature anomalies far from the source with vertical structure very different from that imposed at the source.

In this model, the possibility arises that the temperature and passive tracer plumes point in different directions. Thus it can be misleading to attempt to relate maps of shear to maps of tracers without considering background flow. The degree of correlation of a passive tracer with any of the modes depends crucially on the value of $C$, as well as the location and geometry of the source and Peclet number.

\subsubsection{Solution with application to the South Pacific}

The East Pacific Rise in the South Pacific is thought to be a region of strong geothermal heat flux, because the spreading rate is large there. The presence of hot spots is clearly indicated by the existence of large-scale helium and manganese plumes, with the most extreme values centered at the rise near $110^{\circ} \mathrm{W}$ (Östlund et $a l, 1987$; Klinkhammer and Hudson, 1986). The helium plume extends west near $15^{\circ} \mathrm{S}$ about $7800 \mathrm{~km}$ past $180^{\circ} \mathrm{W}$, where it lies roughly between $10^{\circ} \mathrm{N}$ and $30^{\circ} \mathrm{S}$. South of $25^{\circ} \mathrm{S}$, a manganese plume extends east from the rise, with highest concentrations near $30^{\circ} \mathrm{S}$. Both plumes are at about $2500 \mathrm{~m}$ depth, somewhat above the crest. The importance of these measurements is that they indicate westward middepth low latitude flow, and eastward mid-depth flow farther south to at least $35^{\circ} \mathrm{S}$. The eastward flow probably continues past $35^{\circ} \mathrm{S}$ into the region of the circumpolar current. The observations of mid-depth flow based on He and $\mathrm{Mn}$ are supported by the distribution of hydrothermal precipitates on the seafloor underneath the He and Mn plumes. Edmond et al. (1982) summarize the evidence for both the westward and eastward flow based on seafloor sediment studies, including the distribution of the ratio $\mathrm{Al} /(\mathrm{Al}+\mathrm{Fe}+\mathrm{Mn}$ ), adapted here (Figure 3.4, right column). Hydrothermal deposits are poor in aluminum relative to continental runoff and hence small values of this ratio indicate a strong hydrothermal influence.

Stommel (1982) offered an explanation for the westward flow which involved the westward extension of a vortex generated at the ridge crest by heating. This explanation does not accomodate eastward flow (or any flow) east of the ridge crest, since the $\beta$-plume can only penetrate west in the absence of background flow. Solutions presented here with background flow can have flow east of the forcing region. Furthermore, as has been illustrated in previous sections, the temperature 
perturbation can penetrate upstream and generate a pattern which is not easy to predict. This latter behavior can be compared with observations, as the temperature perturbation at a given level in the model is essentially equivalent to the depth of an isopycnal surface, and maps of isopycnal depth near convenient levels have been presented by Reid (1986).

Observations of tracers and isopycnal depth help constrain the model solution. The relative importance of background flow to long wave speed (C) is constrained by comparing the model temperature to the isopycnal depth. The flow given by the tracers constrains the magnitude of the forcing $(\epsilon / \gamma)$ by comparing flow patterns to total pressure in the model.

A best case run was made for the South Pacific (Figure 3.4). The vertical structure of the forcing represents a stability minimum at mid-depth, thus only even modes are present. The magnitude of the forcing $(\epsilon / \gamma)$ is 0.05 in a region extending from $10^{\circ} \mathrm{S}$ to $20^{\circ} \mathrm{S}(\theta=-0.17$ to $\theta=-0.34) 1200 \mathrm{~km}$ west of the eastern boundary $(\phi=-0.2)$. A weaker forcing elsewhere along the ridge is ignored. This choice is necessary to get mid-depth westward flow near $15^{\circ} \mathrm{S}$, changing to eastward flow farther south. For reference this pattern is illustrated by the distribution of hydrothermal sediment deposits shown along with the solution for pressure at $z=2000 \mathrm{~m}$ (Figure 3.4). If such forcing were to extend southward the eastward flow would be overcome by recirculation near the source. The parameter $\mathrm{C}$ is 6 , which represents a trade-off between the westward extent of the isopycnal depth (and of the westward flow), and their ultimate southeastward trend. It is a combination of localized forcing, eastward background flow, and the latitudinal dependence of westward penetration of the modes which is responsible for westward flow near $15^{\circ} \mathrm{S}$ and eastward flow farther south, in basic agreement with the tracers.

The only region of westward flow is at low latitudes, north of $\theta=-0.3$. Between $\theta=-0.3$ and $\theta=-0.5$ there is some upstream penetration of the temperature anomaly. South of $\theta=-0.5$ all the forced structure goes east.

Isopycnal depths near $2000 \mathrm{~m}$ and $3000 \mathrm{~m}$ adapted from Reid (1986) are overlaid on the temperature anomaly in the middle column. Some distortion was necessary to line up the ridge axis. The isopycnal depth field near $2500 \mathrm{~m}$ is relatively featureless by comparison as expected from a even-mode structure. Only several contours that intersect the region of the rise are shown. The sense of the isopycnal depth variation is consistent with a stability minimum between $2000 \mathrm{~m}$ and $3000 \mathrm{~m}$ depth, and hence agrees with the sign of the temperature anomaly in the solution. The overall patterns agree between $\theta=-0.1$ and $\theta=-0.4$ but the return to the southwest is not too well defined in the data in the upper panel, while the model result is shifted east in the lower panel. No adjustments to the background velocity field were made to improve the correspondence. 

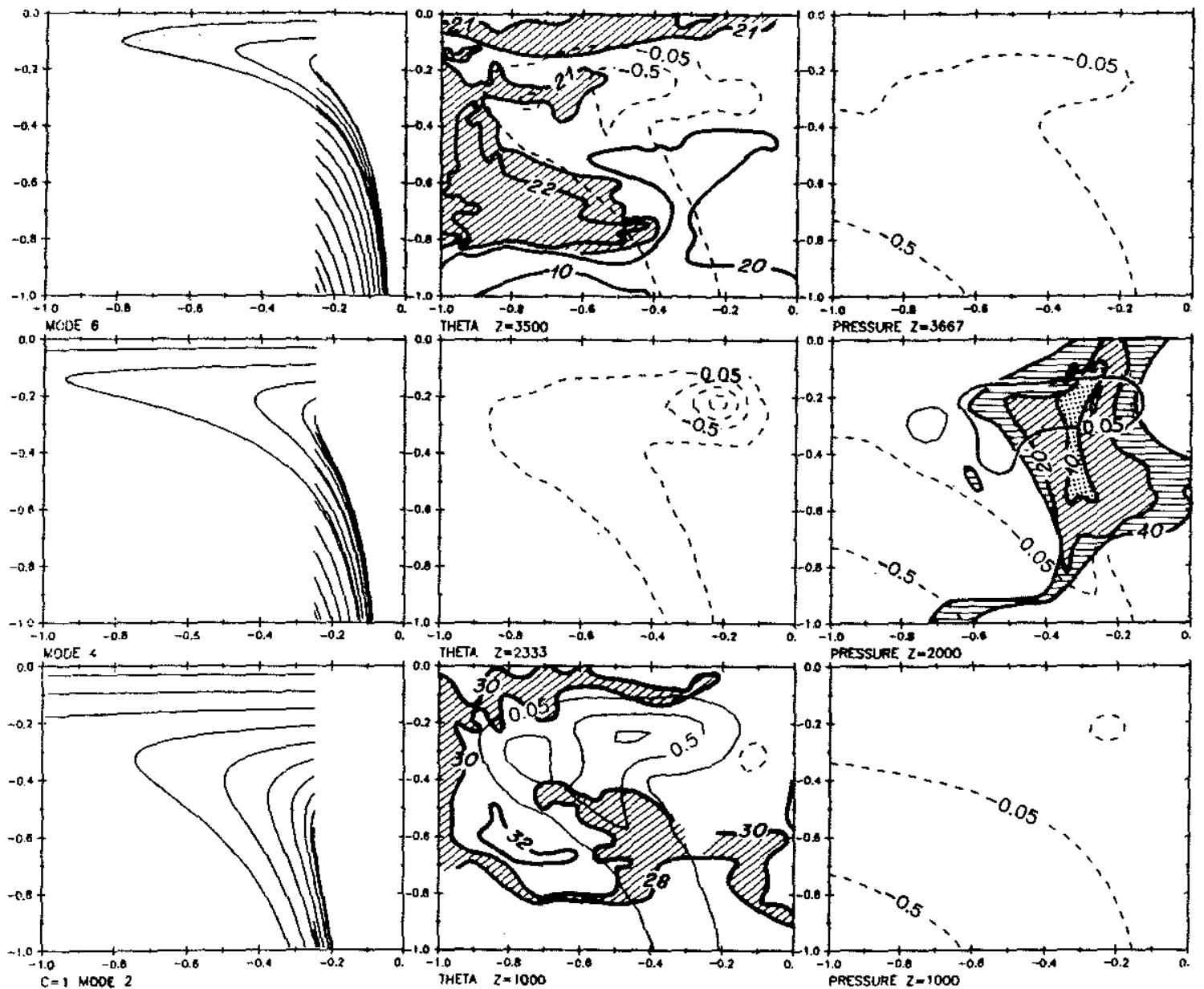

Figure 3.4: Best case solution for the South Pacific, $\mathrm{C}=6, \mathrm{Pe}=7, \epsilon / \gamma=0.05$. Even mode characteristics representing a stability minimum begin at $\phi=-0.2$. Their initial amplitude is nonzero only in the range $\theta=-0.15$ to $\theta=-0.35$. Isopycnal depths near $2000 \mathrm{~m}$ and $3000 \mathrm{~m}$ adapted from Reid (1986) are overlaid on temperature in the middle column, upper and lower panels; the shading of values less than $30 \mathrm{hm}(3000 \mathrm{~m}$, lower $)$, and values greater than $21 \mathrm{hm}(2100 \mathrm{~m}$, upper $)$ is to help separate contours visually. Hydrothermal seafloor deposits indicated by small values of the ratio $\mathrm{Al} /(\mathrm{Al}+\mathrm{Fe}+\mathrm{Mn})$ (adapted from Edmond et al., 1982) showing mid-depth flow are superimposed on pressure at $z=2000 \mathrm{~m}$ (right column). 
In the lower part of the layer underneath the westward flow, the solution shows eastward flow.-This behavior is supported by the distributions of silica and potential temperature at abyssal levels (Reid, 1986, his Figures 62 and 70). These properties are suggestive of such a flow, and furthermore the salinity, oxygen, and shear fields are all at least consistent with it (Reid, 1986, his Figures 57, 60, 61).

The solution shows generally eastward flow near the top of the deep layer. At low latitudes this agrees with Reid's (1986) version of the total flow at $1000 \mathrm{~m}$ depth and with the geostrophic flow at this depth referenced to $3500 \mathrm{~m}$ (his Figures 22 and 23). Property distributions are mostly zonal, but oxygen does show some suggestion of eastward advection (his Figures 26, 30, and 34). In the southwest portion of the basin the solution does not agree with Reid's maps, which are dominated by the subtropical anticyclonic gyre system, shifted south at this depth.

With this solution it becomes clear that the baroclinic structure mapped by Reid (1986) and the flow indicated by the tracer work provide independent information which can be used to predict the forcing and infer the flow in different parts of the basin. This is only true in the context of a model that can calculate the direction of influence of the various vertical components of the forcing. This information is left unused if no account is taken of the dynamically active nature of some quantities (e.g., isopycnal depth) introduced by the forcing. The interplay of active and passive tracers is emphasized in the solutions described here, illustrating some of the ideas thought to be important in the model.

\subsection{Extensions of the Standard Model}

A model of the large-scale influence of a heat source in a simple background flow has been described. The essential features of the standard model are that it is a linear, geostrophic, flat-bottomed model in spherical coordinates. The background flow has no vertical shear. Solutions were sought of the form $\Theta=\sum_{n=1}^{\infty} \Theta^{n} \sin (n \pi z)$. Only the first 3 modes in the sinusoidal vertical temperature structure were retained in the solutions to the temperature equation.

In this section, the results of an examination of some effects which were neglected in the standard model such as higher vertical resolution, different horizontal structure of the source, background vertical shear, and nonlinearity are presented.

\subsubsection{Increased vertical resolution}

When the solution method was given for the standard model it was argued that only three vertical modes were necessary for adequate representation of the solution. The reason for this was the combined effect of decreasing amplitude with mode number 
(generally like $1 / m$ ), and the strong damping of higher modes (like $m^{2}$ ). It was noted that this limited representation of a bottom-trapped source could not resolve the structure. close to the bottom. The influence of the higher modes, governed by their characteristics, is mostly downstream except when the background flow is extremely weak $(C \rightarrow \infty)$. Thus there is the possibility that neglecting these higher modes will suppress a downstream influence and overemphasize the dispersal of the low modes.

Solutions were generated for a variety of cases in which $C$ and the number of modes changed. As many as 50 modes were used, but usually the number was around 10. In all cases the additional modes did not change the large scale pattern of the solution. Near the source the additional modes could have a relatively small effect on the perturbation temperature field. The effect of the additional modes on the pressure perturbation is smaller than that on the temperature because of the $1 / m$ factor in the pressure sum. The three mode solutions seem to adequately represent the large-scale perturbation field. In cases where the initial source vertical structure is highly trapped, resulting in larger amplitudes in the higher modes, and when vertical diffusion is very weak, the near field may not be adequately represented at certain levels. The addition of 1 or 2 extra modes in these cases should be enough to test the representation.

To illustrate the effect of additional modes on the solution a case with 21 modes is shown (Figures 3.5 and 3.6). The Peclet number $P_{e}=100$, and $C=0.05$. The fit to the initial condition of a temperature perturbation decaying exponentially away from the bottom is good above $100 \mathrm{~m}$. It is not much improved overall compared to a 10 mode case, whose fit is good above $200 \mathrm{~m}$. In addition, it is apparent that Gibb's phenomenom is restricting the goodness of the fit near the bottom. If the initial temperature perturbation were zero at the bottom, an odd periodic extension of it would not be discontinuous at $z=0$, and there would be no Gibb's phenomenon. The amplitude of the solution at $2000 \mathrm{~m}$ is slightly larger than the three mode case, because in the latter case the fit to the initial condition happens to be poor near $2000 \mathrm{~m}$. At $\mathrm{z}=667 \mathrm{~m}$ there is a slight increase in downstream influence in the 21 mode case over the 3 mode case. The main effect is clear at $z=300 \mathrm{~m}$, where the 3 -mode solution poorly represents the stronger forcing, while the 21-mode solution represents it well and is stronger than at shallower levels.

\subsubsection{Mediterranean source example}

Solutions have been described that are forced by sources which are supposed to represent geothermal heating near the crest of a mid-ocean ridge. In the standard model the heating is specified as a meridionally confined line source at low latitudes. 


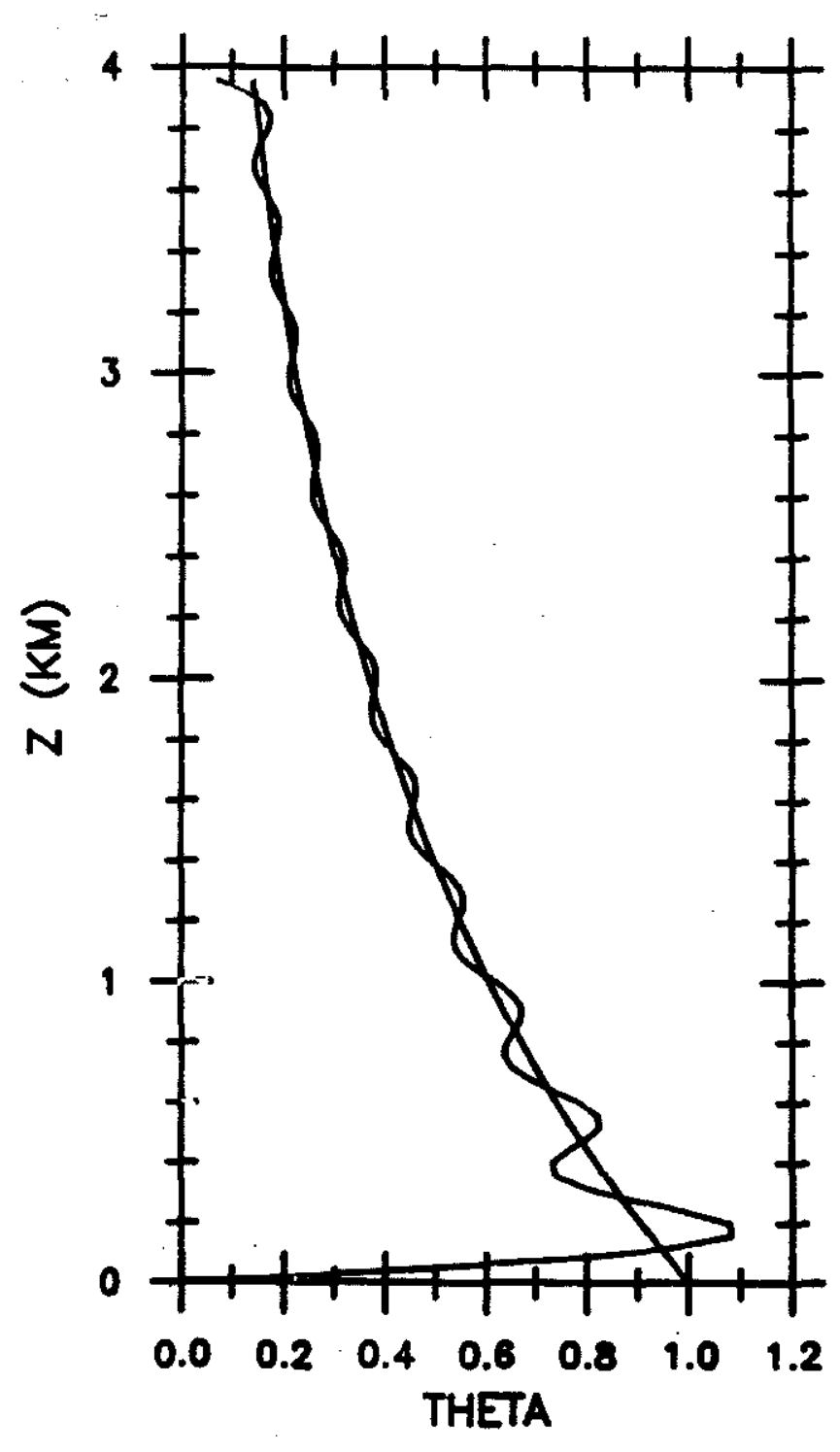

Figure 3.5: Idealized forcing and representation of the vertical structure of the forcing with 21 modes. 

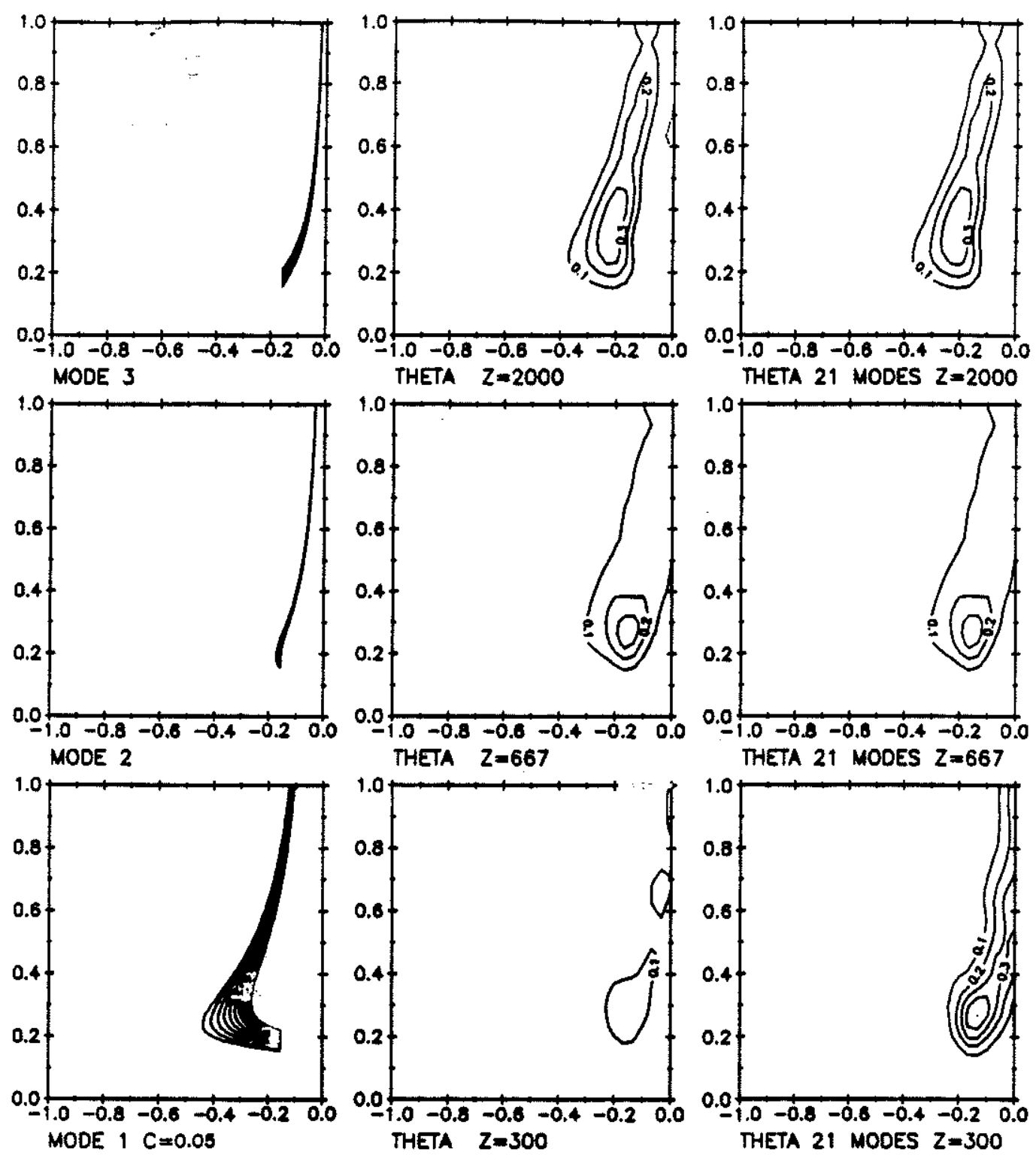

Figure 3.6: Solution for the high vertical resolution case. Characteristics (left); temperature (middle) and pressure (right) at 3 levels. Vertical structure of the forcing from Figure 3.5, $\mathrm{C}=0.05, \mathrm{Pe}=100,21$ modes. 
This horizontal structure strongly influences the solution in the rest of the basin. A long source.(large latitudinal extent) influences a larger area of the basin, and allows more modes to penetrate a given region. In general, a region west of the source recieves a particular mode only from some limited latitude range of the source. Because of this behavior it seems worthwhile to examine solutions with source shapes and locations different from the standard case. The influence of different vertical source structure has been described earlier and resulted from the mode number dependence of phase speed combined with the initial amplitude of each mode.

The density anomaly introduced at mid-depth by the Mediterranean overflow is another example which will be used to illustrate a different source in the model (Figure 3.7). In this case $C$ is fixed at 1 . The extremes of very weak and very strong background flow ( $C$ large and small) are not presented because in those cases the modes essentially overlap, and the resulting pattern is either due west or downstream of the source. These situations are more intuitive and easier to predict than the $C=\mathrm{O}(1)$ case.

The source is at the eastern boundary, centered at $\theta=0.5$ radians, and extending about $500 \mathrm{~km}$ north and south of the center. The vertical structure at the source is a positive density anomaly or cool water perturbation of amplitude $-0.5^{\circ} \mathrm{C}$ exponentially trapped to the top of the layer, with a decay scale of $2000 \mathrm{~m}$. This anomaly represents a bowing upwards of isopycnals in deep water near the outflow, perhaps mimicking the low in steric height evident in Reid's (1981) map. The Mediterranean source is represented only as a density anomaly, the extra mass is neglected. This extra mass could increase the strength of the background flow by increasing the upwelling required to balance mass in the basin. The main effect near the eastern boundary where $\bar{u}$ is weak would be to increase the dispersal of the modes, especially the northward tendency.

Mode 1 is able to reach the western boundary, but modes 2 and 3 quickly turn northwards in the background flow. It is the meridional component of the background flow which determines this pattern. The zonal component is weak near the eastern boundary and plays little role in guiding the characteristics.

The temperature anomaly and total pressure plots are all at levels in the upper half of the $4000 \mathrm{~m}$ thick layer. To make the total pressure plots, $\epsilon / \gamma$ was chosen to be 0.02 . Mode 1 dominates, but there is a substantial northward influence. At $\mathrm{z}=3000 \mathrm{~m}$ the cool tongue appears to bend northwards owing to the higher modes. At $\mathrm{z}=2000 \mathrm{~m}$ there is a weak warm anomaly north of the source because mode 3 is of opposite sign at that level. Similarly the pressure field at $z=2000 \mathrm{~m}$ has a weak anticyclonic tendency to the north of the source, with a northward pointing 

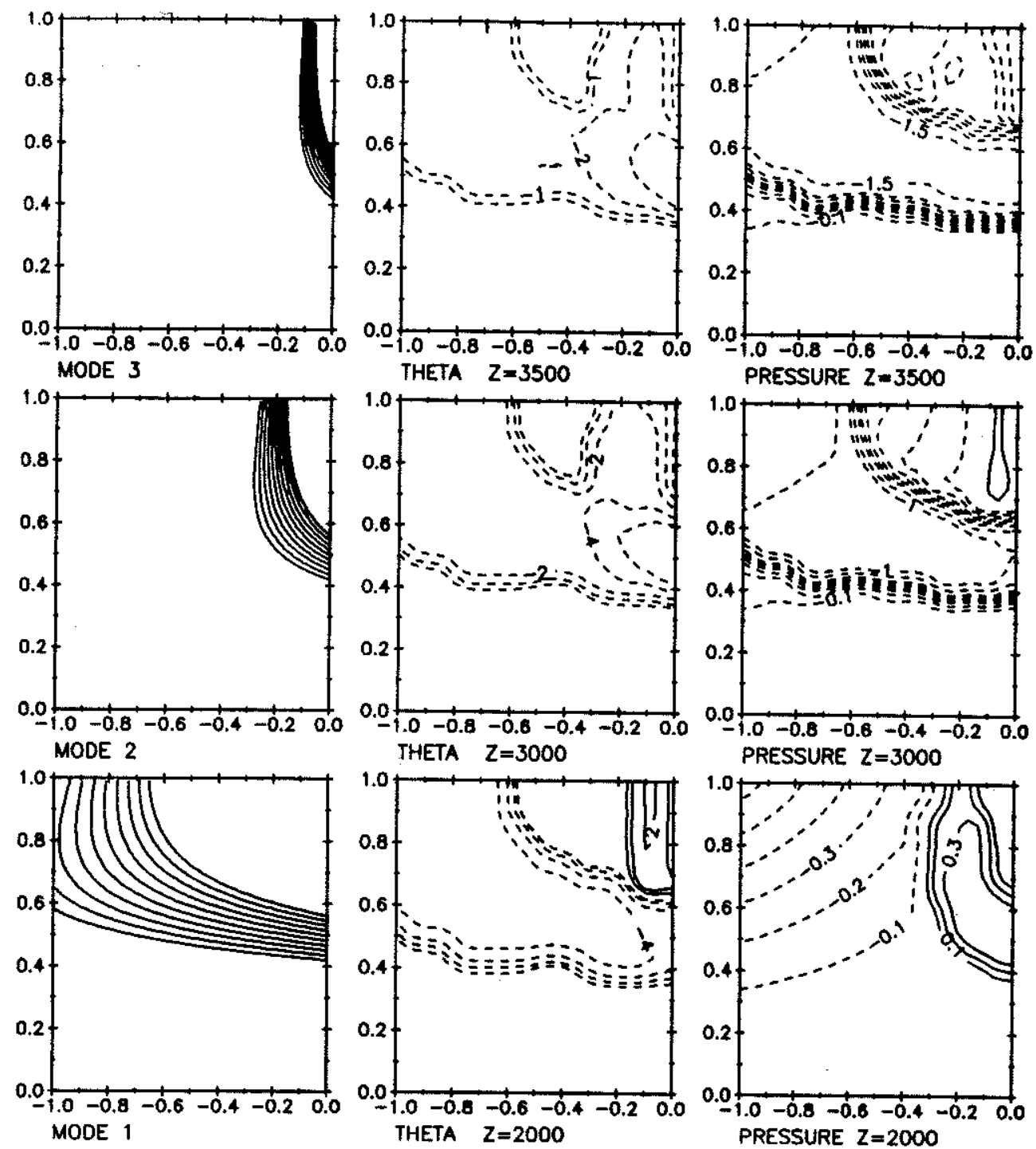

Figure 3.7: Mediterranean outflow example. Characteristics (left); temperature (middle) and pressure (right) at 3 levels. Forcing is a positive density anomaly at the eastern boundary between $\phi=0.4$ and $\phi=0.6, \mathrm{C}=1, P_{e}=1$. 
background pressure field further west, owing to the negative pressure anomaly along mode 1 characteristics at this level. At shallower levels the pressure field is dominated by mode 1 , which adds an increasingly cyclonic tendency towards the top of the layer. In the lower half of the layer the mode 1 pressure anomaly is positive or anticyclonic.

A significant feature of the solution is the northward tendency of the higher modes. This depends only on the presence of a mass source to the basin. In the absence of any other mass sources the mass source of the overflow could not be neglected. The outflow would be expected to flow westward as a jet, but with some influence to the north forced by a northward background flow. Tziperman (1987) has presented a model of the outflow in which he explicitly takes into account the mass source. In his model this mass source gives rise to isopycnal deformations which in turn force vertical motion, stretching the outflow causing it to turn to the north. His three-moving-layer case in which the outflow enters the middle layer would be similar in this model to a forced mode 2 structure with a mid-depth eastern boundary source. Two similar features of the two models are the northward tendency and the ability of diffusion to limit the westward penetration of the outflow by damping out the perturbations.

The basic flow pattern is counterclockwise around the low pressure of mode 1 , changing from eastward in the south to northwestward. Higher modes in the northeast corner turn the flow more towards the northeast at deeper levels until finally causing a weak reversal near $z=2000 \mathrm{~m}$. Thus the main characteristics of the outflow water would be expected to be found to the north and west of the source. This distribution is found to some degree in observations at depths near $1000 \mathrm{~m}$ (Fuglister, 1960; Worthington and Wright, 1970), and in calculations of geostrophic velocity (Saunders, 1982), but a strong influence to the south and west is also clear at deeper levels (Worthington and Wright, 1970). Furthermore the flow calculated by Saunders (1982) for water deeper than $1200 \mathrm{~m}$ is to the south and west and thus opposite to the background flow used in this example. Outflow characteristics south and west of the source could result from horizontal diffusion, which has been neglected here.

\subsubsection{Background Vertical Shear}

Up to now, solutions of the large-scale temperature perturbation equation have been explored with vertically uniform background flow. Vertical shear in the background flow might be expected to influence the solutions by changing the vertical structure of the perturbations. The new vertical structure would have different dispersal characteristics, as has been evident in previous solutions. To investigate the 
effect of vertical shear in the background flow on the distribution of perturbation temperature, the background flow can be expanded in vertical modes and substituted into the linear perturbation equation. The presence of vertical structure in the background flow will couple the modes.

In the standard model the mode equations were uncoupled and each mode's horizontal structure could be solved for separately. When the background flow has vertical structure the linearized horizontal advection terms will involve products of sines and cosines and hence couple the system of equations. This new set is much more difficult to solve because the roots of the cubic equation for the characteristics are not simple expressions. If the shear is assumed to be weak, a perturbation series of decoupled mode equations can be written. The effect of the shear is examined by looking at the first term in this expansion.

Vertical structure in the background field is represented by additional vertical components, as with the perturbations,

$$
\bar{\Theta}(\phi, \theta, z)=\bar{\Theta}_{0 z} \frac{z}{H}+\sum_{n=0}^{\infty} r \bar{\Theta}^{n} \sin (n \pi z)
$$

where $\bar{\Theta}_{0 z}$ is constant and $\bar{\Theta}^{n}$ depends on $\phi$ and $\theta$. As an example, a single additional component is chosen so that the background flow has a $-\cos (\pi z / H)$ structure, as shown. Then the flow is equivalent barotropic and changes from some

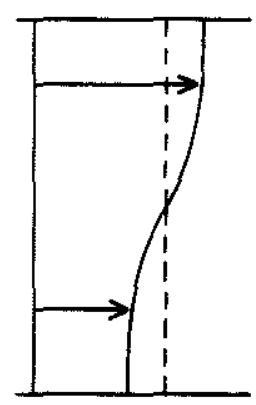

value at the top of the layer to some lower value at the bottom, depending on the sign and amplitude of the shear $r$. In this example, the basin-wide uniform upwelling and the background density field are considered to be given, and are not solved for, since the only purpose is to examine the effect of shear on the perturbations.

Substituting the specified forms of the background and perturbation variables 
into 3.9 , the equation for the $n^{\text {th }}$ mode is

$$
\begin{aligned}
& {\left[\left(\frac{u_{0}}{a \cos (\theta)}-\bar{c}^{n}\right) \Theta_{\phi}^{n}+v_{0} \Theta_{\theta}^{n}\right]} \\
& +\left(\frac{\bar{c}^{1}}{2(n+1)^{2}}-\frac{r u_{0}}{2 a \cos (\theta)}\left(1+\frac{1}{n+1}\right)\right) \Theta_{\phi}^{n+1}+\left(\frac{\bar{c}^{1}}{2(n-1)^{2}}-\frac{r u_{0}}{2 a \cos (\theta)}\left(1-\frac{1}{n-1}\right)\right) \Theta_{\phi}^{n-1} \\
& -\frac{r v_{0}}{2 a}\left(1+\frac{1}{n+1}\right) \Theta_{\theta}^{n+1}-\frac{r v_{0}}{2 a}\left(1-\frac{1}{n-1}\right) \Theta_{\theta}^{n-1}+\frac{\kappa_{v} n^{2} \pi^{2} \Theta^{n}}{H^{2}}=0
\end{aligned}
$$

where

$$
\begin{gathered}
u_{0}=-2 \frac{a w_{0}}{H} \phi \cos (\theta) \quad v_{0}=\frac{a w_{0}}{H} \tan (\theta) \\
\bar{c}^{1}=\frac{\beta g \alpha \bar{\Theta}^{1} H}{a f^{2} \pi}
\end{gathered}
$$

and

$$
\bar{\Theta}^{1}=\frac{r \rho_{0} 2 \Omega a^{2} w_{0} \phi \sin ^{2}(\theta)}{g \alpha H^{2}}
$$

so that $\bar{c}^{1}$ is $O(r)$. The $\bar{c}^{1}$ terms are from the vertical advection of the new background temperature structure, and looks like a modified phase speed, but for the $n \pm 1$ mode. These terms are numerically small because of the $1 / n^{2}$ factor.

The first term in brackets is the original characteristic equation of the standard model. The next four terms couple the $n^{\text {th }}$ mode to the $n \pm 1$ mode. The characteristic equation for this system for an arbitrary number of modes may be written by standard methods. The expression is not very convenient and instead $\Theta^{n}$ can be expanded $\Theta^{n}=\Theta_{0}^{n}+r \Theta_{1}^{n}+r^{2} \Theta_{2}^{n}+\cdots$. The parameter $r$ represents the amplitude of the shear. Since $r=-2 \bar{c}^{1} / u_{0}$, it can be thought of as a small modified phase speed, analogous to the "passive" temperature limit of small $C$. The $O(1)$ characteristic equation is the same as the standard model. The $O(r)$ equation is

$$
u_{0 c}^{n} \Theta_{1 \phi}^{n}+v_{0} \Theta_{1 \theta}^{n}=-\frac{\kappa_{v} n^{2} \pi^{2} \Theta_{1}^{n}}{H^{2}}-S^{n}
$$

where

$$
\begin{gathered}
u_{0 c}^{n}=\frac{u_{0}}{a \cos (\theta)}-c^{n} \\
S^{n}=A^{n} \Theta_{0 \phi}^{n+1}+B^{n} \Theta_{0 \phi}^{n-1}+C^{n} \Theta_{0 \theta}^{n+1}+D^{n} \Theta_{0 \theta}^{n-1},
\end{gathered}
$$

and

$$
\begin{gathered}
A^{n}=\frac{-u_{0}}{2 a \cos (\theta)}\left(\frac{\cos (\theta)}{2(n+1)^{2}}+\left(1+\frac{1}{n+1}\right)\right) \\
B^{n}=\frac{-u_{0}}{2 a \cos (\theta)}\left(\frac{\cos (\theta)}{2(n-1)^{2}}+\left(1-\frac{1}{n-1}\right)\right) \\
C^{n}=\frac{-v_{0}}{2 a}\left(1+\frac{1}{n+1}\right) \quad D^{n}=\frac{-v_{0}}{2 a}\left(1-\frac{1}{n-1}\right)
\end{gathered}
$$


This is the same characteristic equation as the standard model but with a new source term $S^{n}$ and new initial conditions. The new source term might be nonzero anywhere in the basin, so characteristics must begin along both the eastern and western boundaries, from which location they can reach any point in the basin. They have zero initial amplitude. The source term $S^{n}$ is nonzero where horizontal gradients of modes $n \pm 1$ are nonzero. For instance, $S^{n}$ is large across the envelope of characteristics emanating from the heat source. The new source term generates a correction field $\Theta_{1}^{n}$ from zero initial amplitude; this correction field decays away from the new source regions, carrying (small) mode amplitudes into previously unperturbed areas of the basin.

The new source term has to be approximated by a finite difference. The original step-function choice of horizontal heat source structure represents infinite velocities and is numerically unacceptable. This is not a fundamental problem since the resolution can be made arbitrarily high by increasing the number of characteristics. In this example, the heat source was chosen to have a triangular meridional structure, i.e. a linear ramp to a maximum and linear ramp back to zero. This choice spreads the required $\mathrm{O}(1)$ gradients across several gridpoints. It was necessary to triple the number of characteristics used to calculate the $\mathrm{O}(1)$ field from 10 to 30 , and to use 50 of them in the calculation of the correction field, to eliminate false structure. Altogether about 400 integrations were required in each calculation.

The correction field is illustrated together with the $O(1)$ temperature field and characteristics (Figure 3.8a and 3.8b). The correction field is is a result of the interaction of the background vertical structure with the perturbation structure. The amplitude is small, about 0.05 , because the horizontal gradients of the $O(1)$ field are themselves $O(1)$ where the background flow is weak. The strength of the correction is limited because in critical regions where the characteristics for different modes disperse, tending to generate a correction, the characteristics for a particular mode often diverge, counteracting this tendency by reducing horizontal gradients. The amplitude decays along characteristics owing to the damping term.

Some simple approximate analytical expressions for the mode $n$ correction field indicate that it is proportional to the distance that characteristics overlap when they are nearly parallel, and proportional to the mode $n \pm 1 O(1)$ field when they are nearly perpendicular (appendix B). In general, mode 1 crosses mode 2 at a large angle and creates a correction field much like mode 2 itself, but of opposite sign. Near the heat source $(\phi=-0.15)$ the interaction $S^{1}$ is $O(1)$, but it gets multiplied by the interaction time $\Delta \phi / u_{0 c}^{\mathrm{1}}(\Delta \phi$ is the longitudinal extent of the interaction region) which is very small since the characteristic velocity is large, and hence the net effect is small. Modes 2 and 3 are able to build up a significant amplitude and keep it because they tend to remain in each other's interaction region longer than 


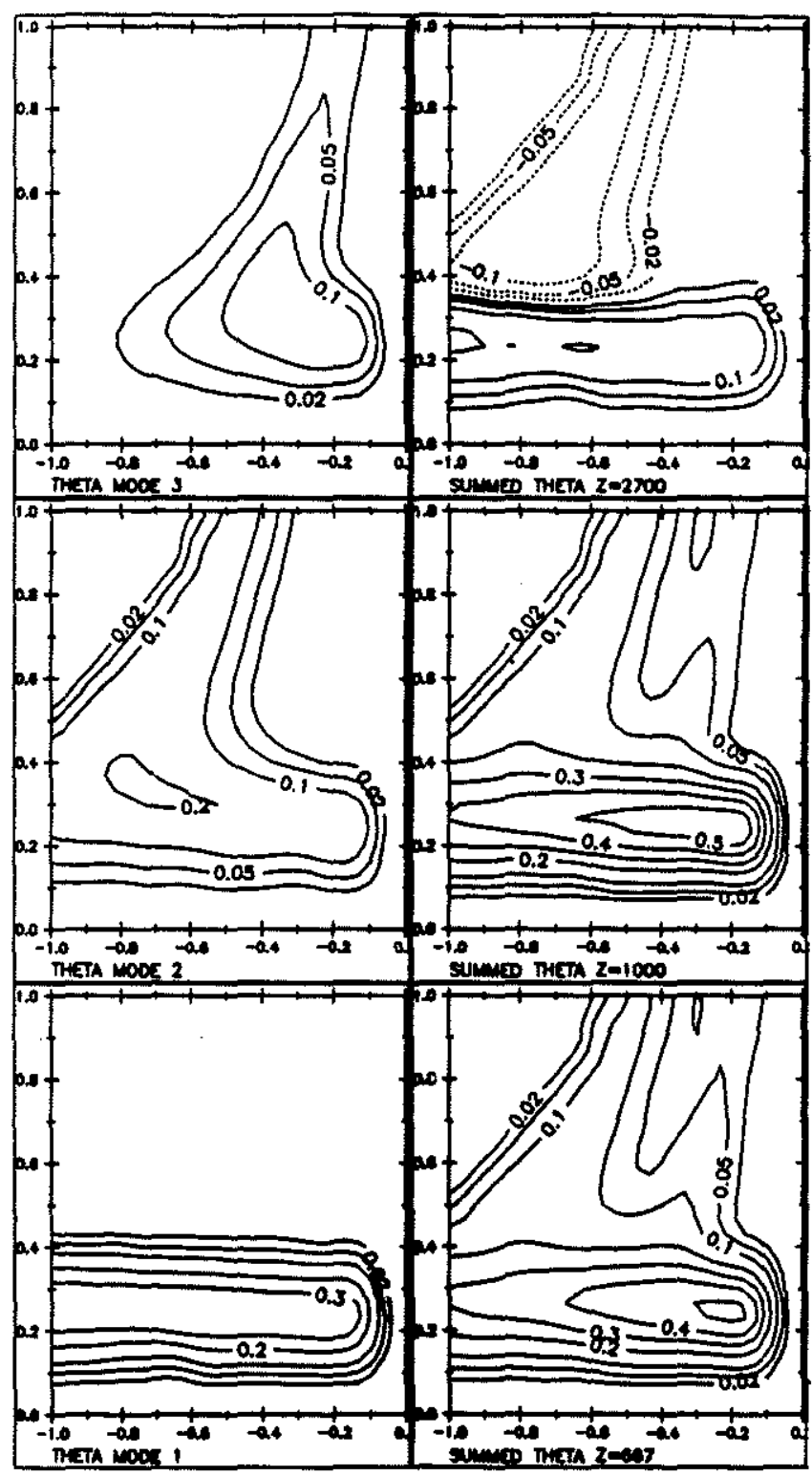

Figure 3.8: a) Solution for a bottom trapped temperature anomaly, $C=1, P_{e}=1$. Temperature perturbation for each mode (left column), and temperature at three levels (right column). 


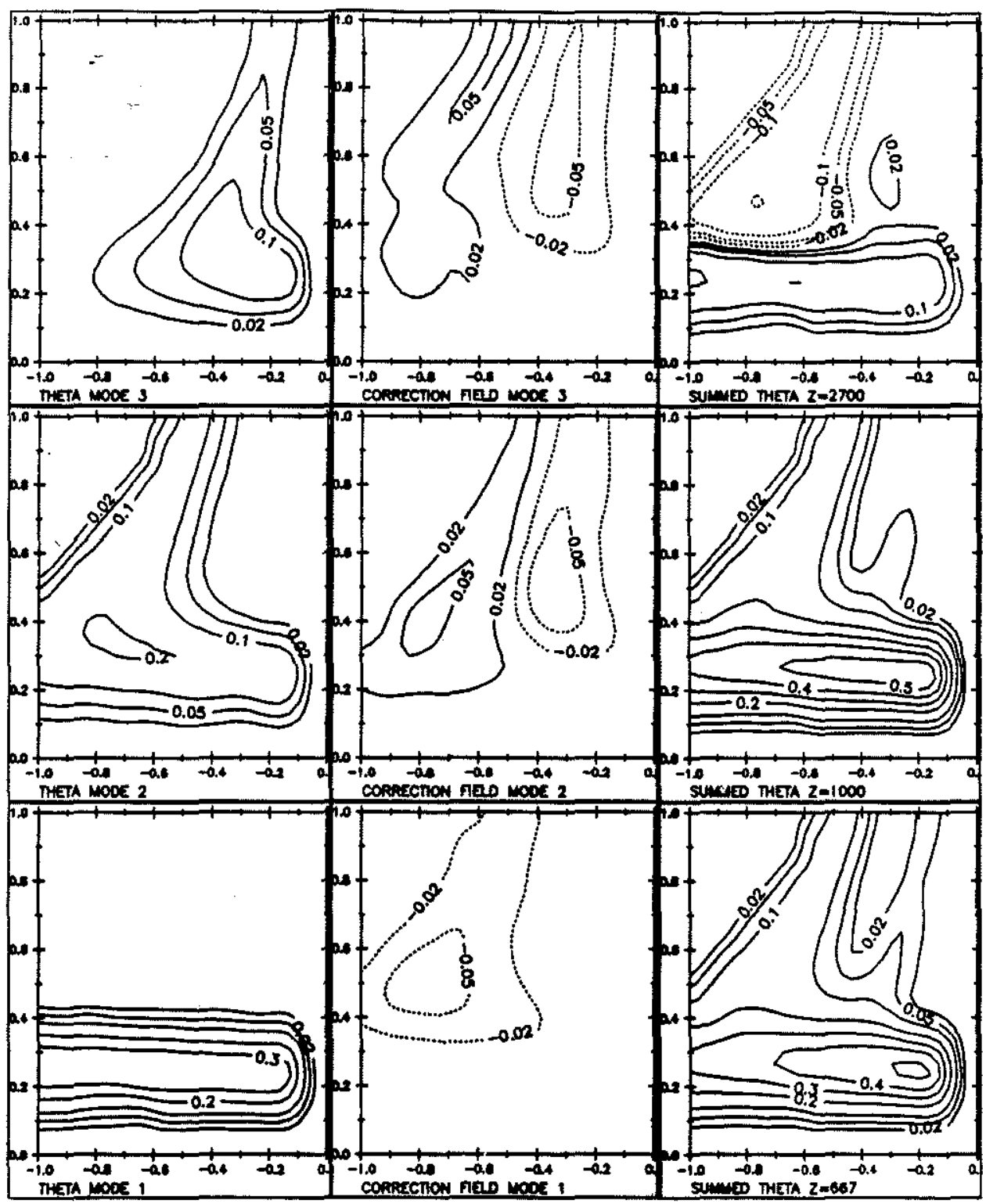

Figure 3.8: b) Solution and correction field for a bottom trapped temperature anomaly with background shear, $C=1, P_{e}=1$. Temperature perturbation for each mode (left column), correction field for each mode (middle column), and summed temperature plus correction for $r=0.3$ at three levels (right column). 
modes 1 and 2. Mode 2 characteristics which nearly parallel the mode 1 plume on the equatorward side cannot generate a mode 2 correction because that coupling coefficient $\left(D^{2}\right)$ is zero. These effects can be used to infer the changes in the total fields. For mode 2 the general effect of the correction is to steepen the eastern side of the plume. For mode 3 the correction acts to shift the pattern westward. Reversing the sign of the shear, so that the flow is weaker near the top, would reverse the sense of the correction.

Although the amplitude of the correction field is small for each mode, it can add up when summed to a more substantial number at certain levels. The maximum change is about $-0.15 r$ at $z=2700 \mathrm{~m}$, resulting from a negative mode 1 and positive modes 2 and 3 near $\phi=-0.8, \theta=0.5$. A value of $r=0.3$ was chosen to maximize the effect of shear, yet the solution does not change much. The conclusion is that although details of the solution may change as a result of the shear, the change is relatively small and predictable.

One lesson from calculating the interaction of background and perturbation fields is the important new role of the horizontal structure of the heat source. Together with the convergence and divergence of characteristics, which compress or expand this structure, it sets the strength of the advection and hence the strength of the interaction.

\subsubsection{Nonlinearity}

The nonlinear terms in the standard model were neglected because it was assumed that the perturbations did not deform the basically flat isopycnals very much. Then the parameter $\epsilon / \gamma$ was small and the lowest order balance was linear. However, a rough scaling of the terms in the full equation with representative values indicates that the horizontal nonlinear terms may be important. In this section, the effect of the nonlinear terms is examined, as before, by a perturbation expansion. Again, this correction can be compared with a weakly coupled system of mode equations. One difference from the previous case is that the equations can be iterated. Iterative solutions for several values of $\epsilon$ are given. Now the temperature field depends on the source strength $\epsilon$ in a more fundamental way, instead of simply being scaled by it. Finally, the importance of the horizontal structure of the heat source will be emphasized.

Keeping the horizontal advection terms $\mathbf{u} \cdot \nabla \Theta$ in the heat equation (3.4), expanding in powers of $a \epsilon / L \gamma$, which represents the strength of the perturbation flow, the $O(a \epsilon / L \gamma)$ equation can be written

$$
\mathbf{u}_{0 c}^{n} \cdot \nabla \Theta_{1}^{n}=-\frac{L n^{2}}{a P e} \Theta_{1}^{n}-C S^{n}
$$


where

$$
\because \quad S^{n}= \begin{cases}\frac{3 J\left(\Theta_{0}^{2}, \Theta_{0}^{1}\right)}{2 \sin (2 \theta)}+\frac{5 J\left(\Theta_{0}^{3}, \Theta_{0}^{2}\right)}{6 \sin (2 \theta)} & n=1 \\ \frac{4 J\left(\Theta_{0}^{3}, \Theta_{0}^{1}\right)}{3 \sin (2 \theta)} & n=2 \\ \frac{J\left(\Theta_{0}^{1}, \Theta_{0}^{2}\right)}{\sin (2 \theta)} & n=3\end{cases}
$$

The $O(1)$ equation is the standard model. Here $J$ is the Jacobian.

The source terms from nonlinearity come into play when the characteristics of one mode are not parallel to those of another. Thus the largest effect occurs when $C$ is $O(1)$. In both the pure $\beta$-plume case $(C \rightarrow \infty)$, and the passive tracer case $(C \rightarrow 0)$ the terms are smaller. In the following $C$ is fixed at 1.

The characteristics and temperature of the $O(1)$ field and the correction field are illustrated (Figure 3.9). A triangular meridional source structure was used, as discussed in the previous section. Only every third characteristic is plotted. The effect on mode 1 is to steepen it in the region where mode 2 characteristics turn northward. The mode 2 correction is largest, with an amplitude as high as 0.9 -much larger than the $O(1)$ mode 2 field. The overall tendency is for mode 2 to be made higher and steeper, counteracting the initial divergence of its characteristics. The mode 3 correction is about twice the amplitude of the $O(1)$ field, and tends to broadly amplify it.

The pattern of the correction field for mode 1 can be understood physically by considering the clockwise flow circulating at mid-depth owing to the mode 2 high pressure perturbation. This flow goes up the mode 1 gradient near the heat source, and down-gradient further west. For mode 1 characteristics penetrating from the east, this heat flux divergence and convergence gives rise to an isolated negative correction field.

For the mode 2 correction field, the dominant horizontal advection term is the product of mode 3 velocity with mode 1 temperature, which has the structure of positive mode 2. A clockwise circulation around the $O(1)$ mode 3 perturbation is up-gradient and hence cooling immediately west of the source, and down-gradient further to the west. The correction is negative in the south because $\Theta_{y}^{1}$ is of opposite sign there. The amplitude of the mode 2 correction is larger because mode 3 is more "perpendicular" to mode 1 and because mode 2 is slower and remains in an overlapping mode region longer. The tendency for mode 2 characteristics to carry this correction along their path away from the interaction region is more noticeable in the mode 2 correction field, together with its decay.

Mode 2 velocity advects heat into mode 3 north and south of the center of the mode 1 plume, owing to the product of mode 2 velocity and mode 1 temperature. Since the strongest effect is near the heat source, mode 3 characteristics carry this correction in a pattern resembling the $O(1)$ mode 3 field itself. 


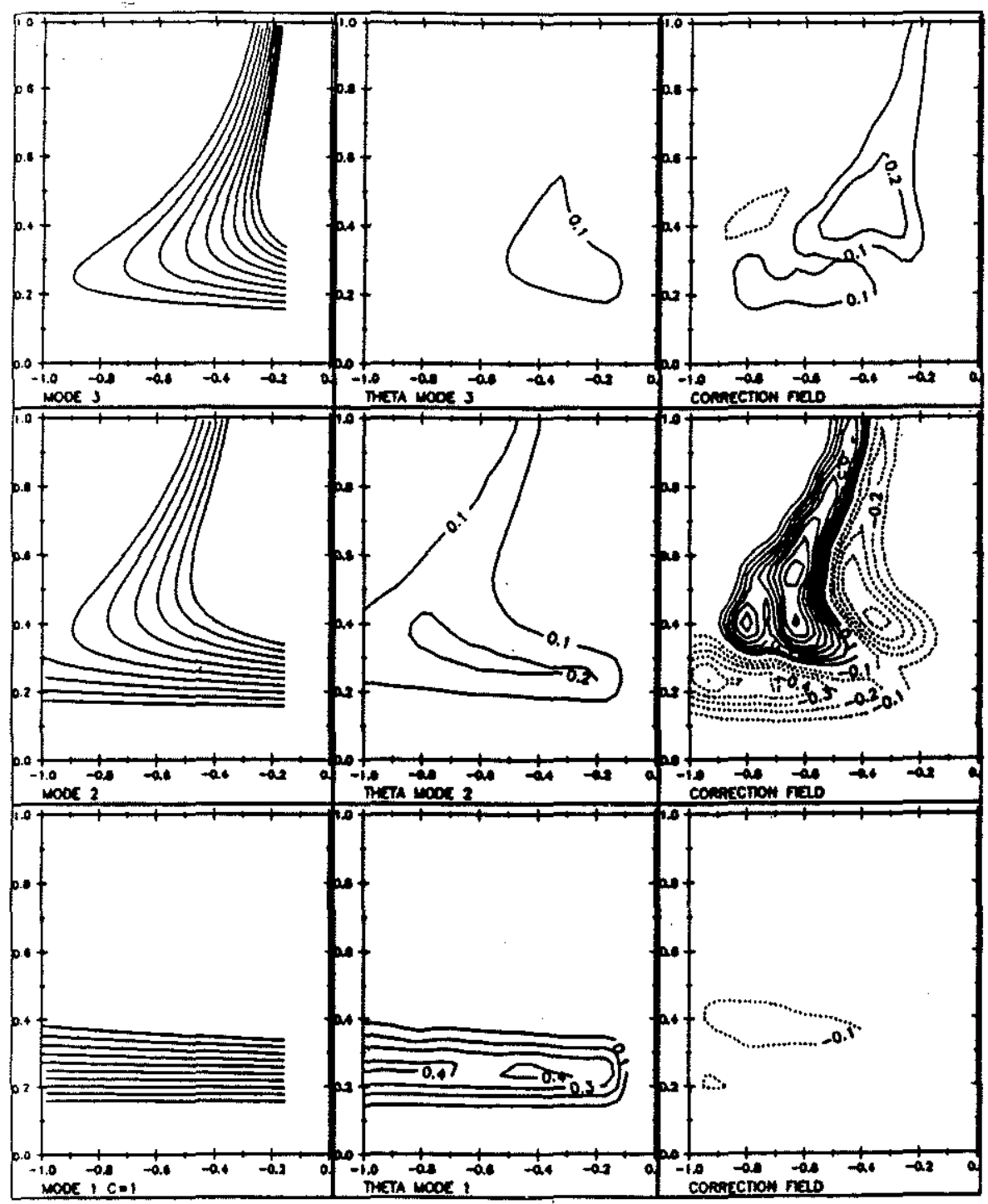

Figure 3.9: a) Characteristics (left column), temperature perturbation (middle column), and correction field (right column) for nonlinear effect. $\mathrm{C}=1, \mathrm{Pe}=1$. 


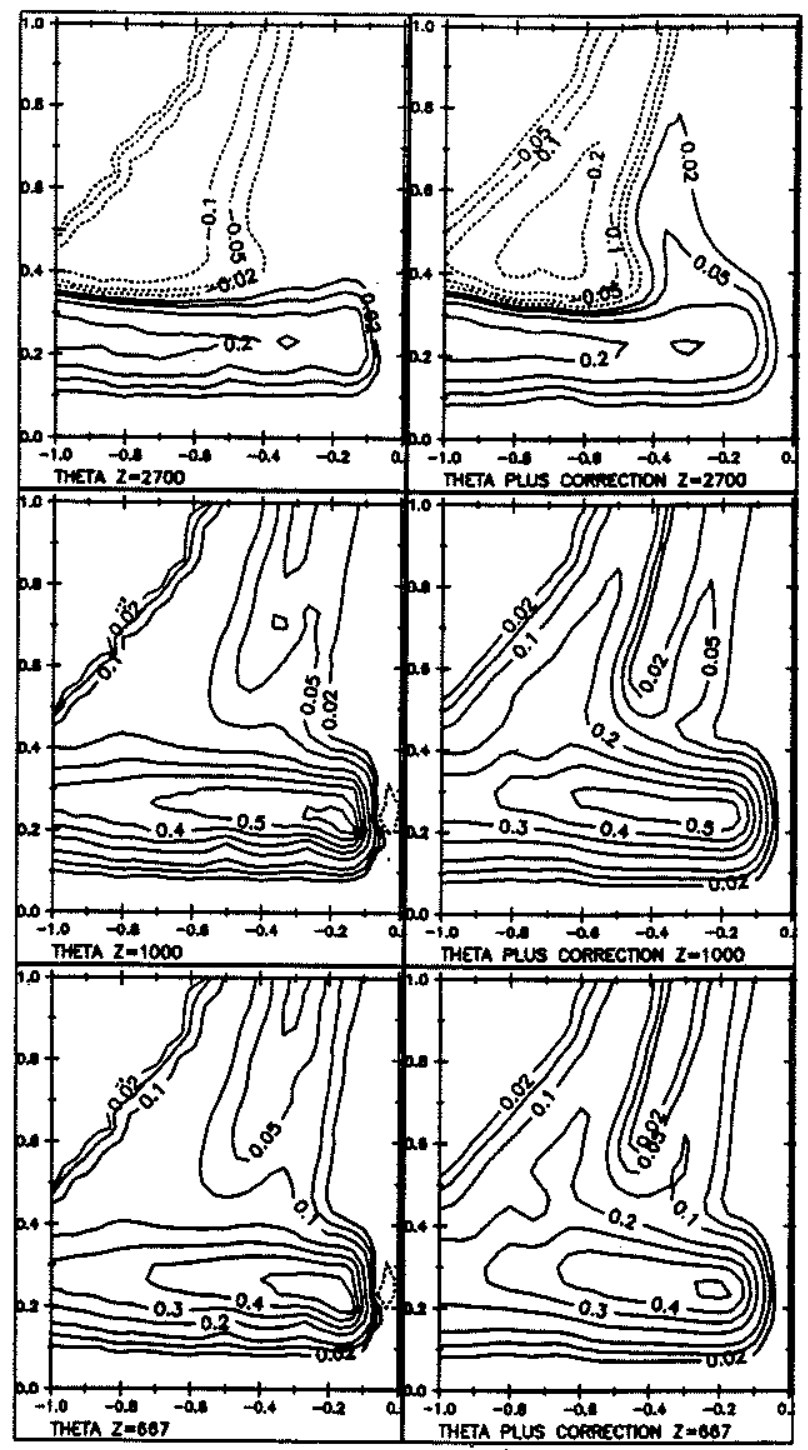

Figure 3.9: b) Total temperature field without nonlinear effect (left), and including nonlinear correction (right), $a \epsilon / L \gamma=0.3$. 
In physical space the effect of the correction fields depends on the relative contribution of each mode at a given level. The dominant effect comes from the mode 2 correction, and the overall modification is to increase horizontal gradients and therefore vertical shear in the northern part of the plume. The nonlinear terms affect the energy distribution among the three modes. It is clear that the higher modes, modes 2 and 3, gain energy, while mode 1 loses it. These exchanges of energy will appear in solutions presented in the next section.

\section{Iterative Solutions}

Once the correction field has been calculated from the $O(1)$ solution, it can be multiplied by $C a \epsilon / L \gamma$ and they can be summed. Then a new correction field can be calculated from this improved estimate of the solution. The whole process of calculating a correction field and adding can be repeated, building up a sequence of solutions. The hope is that after a few iterations they stop changing and represent an approximate solution. The solution is approximate because the finite difference representation of nonlinearity restricts resolution to about $200 \mathrm{~km}$.

The first 3 iterations are shown for $\varepsilon=C a \epsilon / L \gamma=0.1$ (Figure 3.10). What is shown is the sum at each stage; $C$ and $P e$ are fixed at 1 . After the third iteration, nothing changes. The runs may be summarized by plotting modal energy, defined as the mean square value of amplitude (Figure 3.11). Iteration 0 is the solution to the linearized equation. After the initial growth of modes 2 and 3 and decay of mode 1 during the first 3 iterations, there is no further change in energy. The changes agree with the tendency in the correction fields, amplifying modes 2 and 3 , and decreasing mode 1 slightly. Apparently more heat is advected away from the heat source to raise the total energy when nonlinearity is included. In the next case $\varepsilon=0.15$. Energy changes occur up to the sixth iteration. Finally at $\varepsilon=0.2$ the solution does not converge.

The dimensional amplitude depends on the value of the background vertical density gradient strength $\gamma$. For a $5^{\circ} \mathrm{C}$ vertical temperature change in deep water, $\gamma$ is about $10^{-3} \mathrm{~g} \mathrm{~cm}^{-3}$. Then for the non-convergent value $\varepsilon=0.2, L=10^{8}$, $\epsilon=3 \times 10^{-5}$. The resulting temperature deviation is $\epsilon / \alpha=0.15^{\circ} \mathrm{C}$. As expected, this value is somewhat high for large-scale temperature anomalies near ridge crests, which are more typically near $0.05^{\circ} \mathrm{C}$.

The iterated solutions indicate that there is a limit to the source strength above which the basic assumptions of the model break down. They also suggest that for weak nonlinearity, the original solutions to the linearized problem are a good representation of the large-scale temperature distribution resulting from a heat source.

\section{Weak Coupling}

Mode 1 remains fairly passive in the weakly nonlinear solutions. It is therefore 


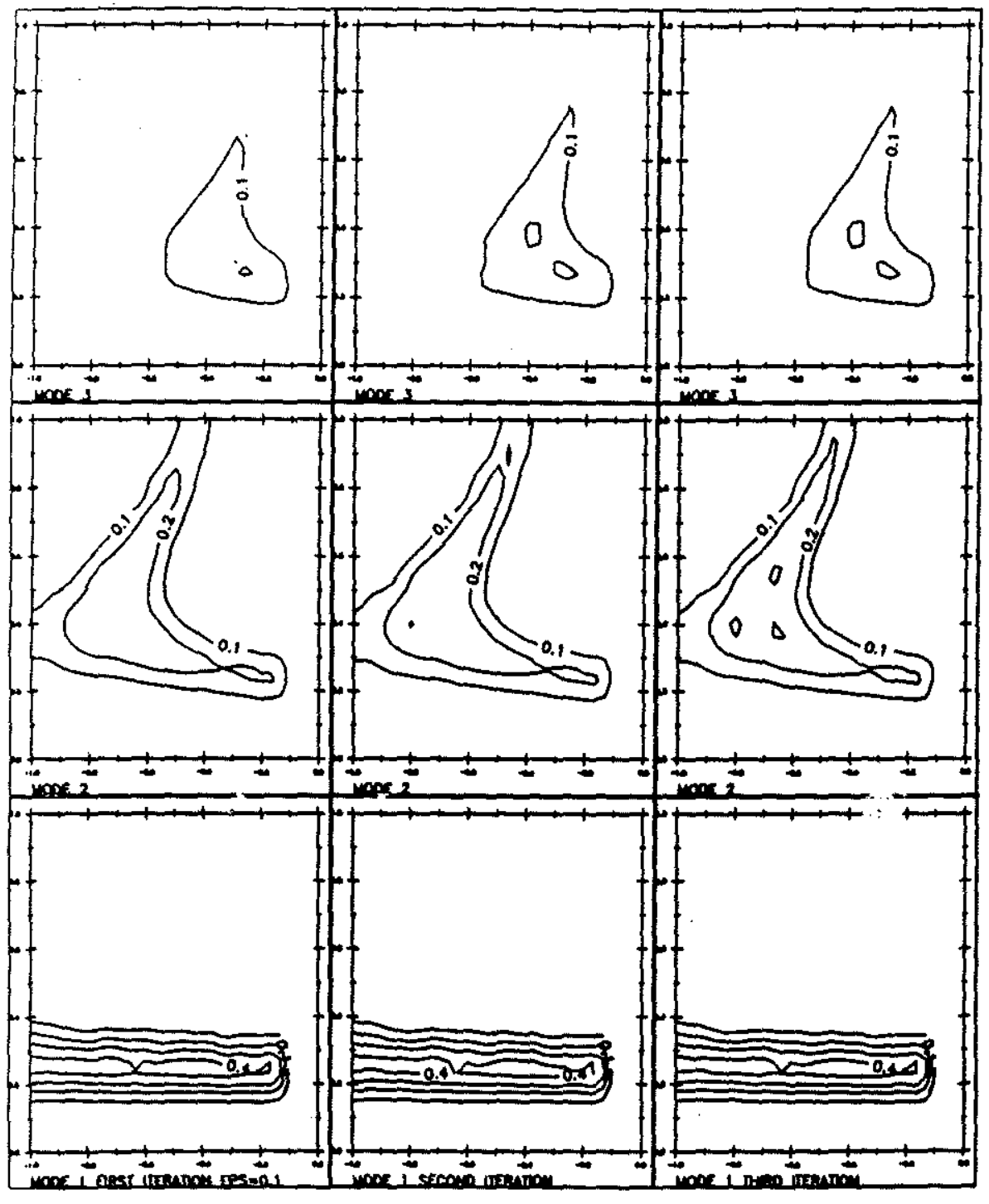

Figure 3.10: Temperature field of iterative nonlinear solutions of the chacteristic equation. Iteration 1 (left), iteration 2 (middle), iteration 3 (right). 

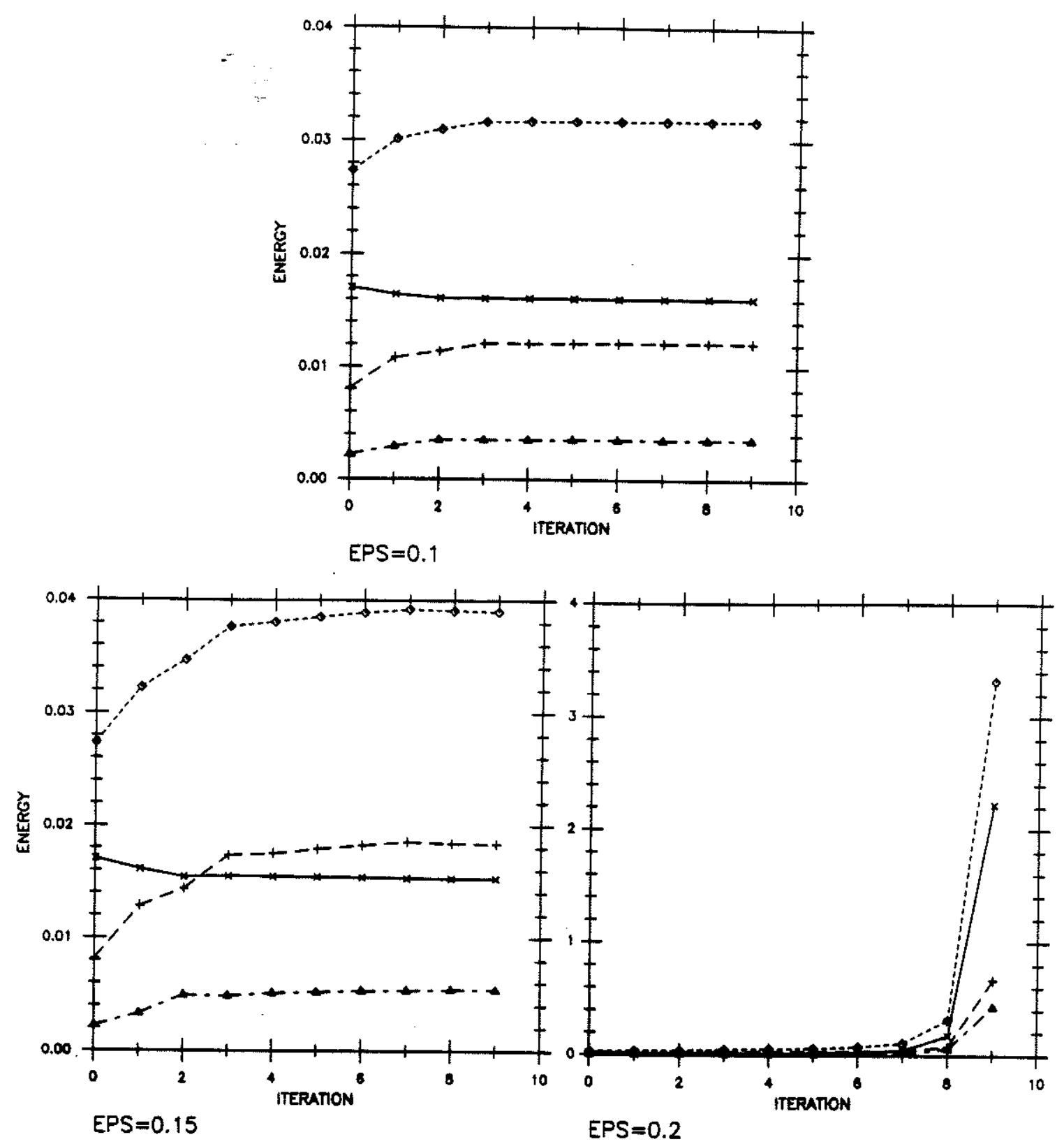

Figure 3.11: Energy at each iteration. For each plot the topmost curve is total energy, second from top is mode 1 energy, third is mode 2 energy, fourth is mode 3 energy. a) $\varepsilon=0.1$, b) $\varepsilon=0.15$, c) $\varepsilon=0.2$. 
tempting to neglect the coupling of mode 1 to modes 2 and $3\left(S^{1}\right)$, to simplify the calculation of the characteristics. This approximation leaves the system linear, but still coupled,-like the background shear case. It is equivalent to assuming that mode 1 has a very fast time scale, i.e. large characteristic velocity. Then the system becomes, neglecting diffusion,

$$
\begin{gathered}
\mathbf{u}_{0 c}^{1} \cdot \nabla \Theta^{1}=0 \\
\mathbf{u}_{0 c}^{2} \cdot \nabla \Theta^{2}+\varepsilon a\left(\Theta_{\phi}^{3} \Theta_{\theta}^{1}-\Theta_{\phi}^{1} \Theta_{\theta}^{3}\right)=0 \\
\mathbf{u}_{0 c}^{3} \cdot \nabla \Theta^{3}+\varepsilon b\left(\Theta_{\phi}^{1} \Theta_{\theta}^{2}-\Theta_{\phi}^{2} \Theta_{\theta}^{1}\right)=0
\end{gathered}
$$

where

where $\varepsilon=C a \epsilon / L \gamma$.

$$
a=\frac{4}{3 \sin (2 \theta)} \quad b=\frac{1}{2 \sin (2 \theta)}
$$

The mode 1 temperature can be solved for everywhere in the basin as before, and substituted into the mode 2 and mode 3 equations, which then become linear. Characteristics begin at the heat source. They are defined by the equation for their slope $s$, which is obtained by the condition that some linear combination of the equations exists in which changes in the mode amplitudes occur in a specific direction (Whitham, 1974). It is

$$
\left(\bar{v}_{0}^{2}+\varepsilon^{2} a b\left(\Theta_{\phi}^{1}\right)^{2}\right) s^{2}+\left(\varepsilon^{2} 2 a b \Theta_{\theta}^{1} \Theta_{\phi}^{1}-u_{0 c}^{2} \bar{v}-u_{0 c}^{3} \bar{v}\right) s+\varepsilon^{2} a b\left(\Theta_{\phi}^{1}\right)^{2}+u_{0 c}^{2} u_{0 c}^{3}=0 .
$$

In the nonlinear solutions, the modal amplitudes were always zero east of the heat source for $C=1$. If this is assumed to still hold, the limit $\Theta_{\phi}^{1} \rightarrow 0$ can be examined to further simplify the problem, since mode 1 is almost zonal. Thus for $\varepsilon \ll 1$, the characteristic slopes are

$$
s^{+}=\frac{u_{0 c}^{2}}{\bar{v}}+\frac{\varepsilon^{2} 36\left(\Theta_{\theta}^{1}\right)^{2}}{15 C} \quad s^{-}=\frac{u_{0 c}^{3}}{\bar{v}}-\frac{\varepsilon^{2} 36\left(\Theta_{\theta}^{1}\right)^{2}}{15 C}
$$

The tendency is for a compression of the original mode 2 envelope of characteristics, because of the addition of some eastward velocity. Conversely the mode 3 envelope is expanded where it overlaps mode 1 . The compression of mode 2 is at least qualitatively consistent with the steepning tendency of the correction field (Figure 3.9a). The expansion of mode 3 is not obvious in the figure, but mode 2 characteristics do carry some mode 3 amplitude to the west of the original mode 3 pattern, below the contour level.

The discriminant of the characteristic (slope) equation (3.28) west of the source may be calculated. When it is negative somewhere the system is no longer 
hyperbolic and it is not appropriate to consider characteristics. The discriminant turns out to be positive for $\varepsilon$ less than about 0.15 . This seems to correspond approximately with the value for which the iterative solutions stopped converging. The correspondance is not exact, but it suggests that a physical criterium for convergence is that the system be able to propagate information into any region of the basin.

\subsection{Conclusion}

The large-scale distribution of properties are generally consistent with the deep circulation of the Stommel and Arons (1960) model. Recent tracer measurements, for example in the South Pacific, suggest other components of flow which may be forced by heating at the mid-ocean ridges. In this chapter a model has been developed for the influence of a heat source in an ocean basin, with the idea that any flow forced by heating would have to overcome the flow forced by a general upwelling. The ability of a background flow to overcome the westward tendency of large-scale temperature perturbations and to break apart the forced vertical structure has been examined. Part of the behavior of the solutions is that the location of temperature anomalies need not be related to that of passive tracers. Perturbations of a background vertical temperature gradient can penetrate westward against a background flow; however, since tracers introduced at the source typically do not have a background vertical gradient their influence must be purely downstream. The two governing parameters are the Peclet number and $\mathrm{C}$, the ratio of long wave speed to background flow strength. This latter parameter can be related to observed density fields, while the forcing strength can be related to observed tracer patterns indicating total flow. This situation was illustrated with an example using observations from the South Pacific, and will also be apparent in numerical solutions presented in chapter 4 .

In the model, bottom depth variations were ignored. The effect of bottom topography such as a ridge with a east-west slope could be to significantly modify the direction of influence of the temperature perturbations. This may occur because just as planetary beta gives the modes their tendency to penetrate west, topographic beta resulting from the flow at the bottom (Gill, 1982), might steer the modes along the ridge to the north or south of the flat-bottomed path.

The effect of higher vertical resolution was explored, as was a different horizontal structure representing the Mediterranean outflow. Extensions of the standard model have been made to include background structure and nonlinear terms in the temperature equation. Within the range of validity of the techniques used to make the extensions, the standard model solutions are not much modified. In the next chapter numerical methods will be used to solve a more complete set of equations 
in a closed basin to check and extend these results. 


\title{
Chapter 4
}

\section{A Numerical Simulation of Geothermal Heating}

\begin{abstract}
Computer generated solutions to large-scale equations in a basin are examined to see if their behavior is similar to that of the analytical model presented in Chapter 3. The advantage of the numerical model is that the strengths of the forcing and background shear are not restricted. In the numerical model, the main effect of strong heating is that the warm plume west of the heat source narrows latitudinally, and that a large component of velocity exists along the core of the plume. For a typical deep water stratification, the pattern occurs when the strength of the heating is several times that estimated to be a typical real average value. For strong background flow, the plume breaks apart into its modal constituents and isotherms flatten back toward the basic state. Background shear with a large vertical scale modifies the solutions, but the effect is not great. The numerical model agrees with the basic ideas present in the analytical model.
\end{abstract}

\subsection{Introduction}

The standard model and extensions presented in the previous chapter were based on simplified physics. The heat source was represented as a fixed temperature anomaly, and no boundary conditions were imposed around the edge of the basin. Thus the solutions were meant to apply in an interior region, outside of any boundary layer around the source or next to a wall, where gradients may be large. Furthermore the strength of the source was thought to be restricted. There was a concern that method of solution by characteristics might break down before the model itself did (section 3.4.4).

The basic motivation, therefore, for examining the output of a numerical model was to see if there is a general agreement between solutions of the analytical model and those of a more complete set of equations solved in a closed system. The numerical solution contains all the boundary layers required near a source and 
walls, and is restricted in source strength only by the condition that there be a steady solution.

A new numerical model became available which made this comparison practical. This model was designed by R. Salmon; no results from this model have yet been published. The key elements of the model are that the momentum equations are steady and linear, with Rayleigh damping in all 3 velocity components, and that the temperature equation is nonlinear, with vertical and horizontal diffusion. A linear analytic version of this model has been described by Salmon (1986), with application to the general circulation. The goal is not to compare this model directly to nature, but to examine and extend the physics of the analytical model.

This numerical model was designed to study the general circulation, including an Ekman layer, thermocline, and abyssal flow, i.e., wind and buoyancy driven circulation. For this application, the model was modified to simulate only the abyssal layer. A background flow was prescribed throughout this layer and temperature was specified at the boundaries. The simulation of an abyssal layer was necessary to get adequate vertical resolution in the region of interest. The details of the model and its modification are discussed next. In modifying the numerical model to represent only an abyssal layer some sacrifice of power and generality has to be made. The only justification for this is to keep focused on the goal of this study.

\subsection{Geometry, Equations, and Forcing}

The geometry is a rectangular box $4000 \mathrm{~km} \mathrm{x} 4000 \mathrm{~km} \mathrm{x} 4 \mathrm{~km}$. There are 10 levels or 9 modes on a $20 \times 20$ grid. The advection of heat is done in physical space while the pressure field is solved for in terms of vertical modes. The equations solved by the numerical model are

$$
\begin{gathered}
r u-f v=-\phi_{x}+\tau_{z}^{x} \\
r v+f u=-\phi_{y}+\tau_{z}^{y} \\
r w=-\phi_{z}+g \alpha \theta \\
u_{x}+v_{y}+w_{z}=0 \\
\theta_{t}+u \theta_{x}+v \theta_{y}+w \theta_{z}=K_{v} \theta_{z z}+K_{H} \nabla^{2} \theta+\frac{Q}{\rho C_{p}} .
\end{gathered}
$$

In these equations, $(x, y, z)$ are cartesian coordinates positive in the east, north, and up directions. The velocity field in these coordinates in $(u, v, w)$. The other variables are potential temperature $(\theta)$ and pressure $(\phi)$ divided by a constant density $\rho_{o}$. The stress divergence $\tau_{z}$ and the heating $\mathrm{Q}$ are both given functions of $\mathrm{x}, \mathrm{y}$ 
and z. Also, $g$ is gravity, $\alpha$ is the thermal expansion coefficient divided by $\rho_{o}$, and $K_{v}$ and $K_{H}$ are vertical and horizontal turbulent heat diffusion coefficients.

With friction $r$ and $f=f_{0}+\beta y$, where $f_{0}$ is the Coriolis parameter evaluated at the central latitude of the box, the vorticity equation is

$$
r \zeta+\beta v=f w_{z}+\tau_{z x}^{y}-\tau_{z y}^{x},
$$

where $\zeta=v_{x}-u_{y}$. The velocity components are

$$
\begin{gathered}
u=\left(r^{2}+f^{2}\right)^{-1}\left[-f \phi_{y}-r \phi_{x}+f \tau_{z}^{y}+r \tau_{z}^{x}\right] \\
v=\left(r^{2}+f^{2}\right)^{-1}\left[f \phi_{x}-r \phi_{y}+r \tau_{z}^{y}-f \tau_{z}^{x}\right]
\end{gathered}
$$

The vorticity equation can be written as

$$
r^{2} \nabla \cdot f^{-2} \nabla \phi+\frac{r \beta}{f^{2}} \phi_{x}=-\phi_{z z}+g \alpha \theta_{z}+r \hat{z} \cdot \nabla \times\left(\frac{\tau_{z}}{f}\right)
$$

for small $r$, where $\hat{z}$ is the unit vector in the vertical direction. Given some initial condition the temperature at a new time can be found, and then this equation can be used to find the pressure. There is no normal flow at the boundaries, and temperature is specified to be linear in $z$ on all sides. The latter condition imposes a basic stratification like that of the analytical model. At the top and bottom boundaries $w=0$ and temperature is independent of horizontal position, so the steady solution satifies $\rho_{z z}=0$, as in the analytical model.

The interior solution obtained by setting $r=0$ is $\phi_{z}=g \alpha \theta$, that is, the hydrostatic relation. If this solution does not satisfy the no normal flow condition at the boundaries, then higher order terms will become important. In the absence of stress, the basic balance in 4.6 is $\beta v=f w_{z}$. These two balances just express the smallness of $w$ in the interior, where the flow is basically hydrostatic and geostrophic.

The representation of a localized heat source in a large-scale model can only be very schematic. The spacing between gridpoints is $200 \mathrm{~km}$, which is wider than the median valley of any mid-ocean ridge. Heating is represented in the model in terms of 3 modes:

$$
Q=Q_{s}\left(Q^{1} \sin (\pi z)+Q^{2} \sin (2 \pi z)+Q^{3} \sin (3 \pi z)\right) \delta\left(x-x_{\circ}\right) \Pi(y) .
$$

In this expression, $Q_{s}$ sets the overall scale of the heating and the $Q^{i}$ set the individual forced-mode amplitudes relative to unit forcing. The horizontal structure is a line source along $\mathrm{x}=\mathrm{x}_{0}$ with a step-function meridional structure of variable extent. In the solutions illustrated below, the horizontal structure of the forcing is clear. A few preliminary runs were made with $Q$ proportional to a delta function 
in $z$; however, the above form was settled on for easier comparison to the analytical solutions.

The magnitude of the heat source scale $Q_{s}$ comes from an estimate of the average geothermal heat flux over a segment of a mid-ocean ridge's median valley. The distribution of heat flux, made up of conductive and hydrothermal components, along the crest of mid-ocean ridges is not well known. An estimate of the total average heat flux made up of a conductive component plus a hydrothermal component is of order $1 \mathrm{~W} / \mathrm{m}^{2}$ (Chapter 1 ). From this value $Q_{s}$ turns out to be about 1 in the units used in the model $\left(3 \times 10^{-9} \mathrm{~W} \mathrm{~cm}^{-3}\right)$.

Friction has an important role in the numerical model, absorbing interior flow at walls and near the heat source, and helping to smooth out numerically unacceptable gradients. It has a diffusive-like effect on temperature since $r \zeta \sim r \nabla^{2} \theta$ by the thermal wind, and thus tends to close isotherms around the heat source. At meridional "Stommel" boundary layers the thickness is $r / \beta$, while zonal boundary layers are wider, $(r / \beta)^{1 / 2}$, and their thickness increases to the west (Salmon, 1986). To approach the case of maximum westward penetration of the heating $r$ was reduced below the value strictly required for a one gridpoint wide east-west layer $(\beta \Delta x$, where $\Delta x$ is the grid-spacing). However, for numerical stability this boundary layer has to be resolved. The resolution is accomplished implicitly, because the upstream differencing scheme introduces zonal diffusion over exactly this scale. The result of lowering the explicit value of $r$ is, then, to shrink the wide zonal boundary layers which emanate from the corners of the heat source by about a factor of two, from 4 grid-spacings to 2 grid-spacings. Without this correction the boundary layers are so wide that they are important over too large a region of the interior west of the source.

The choice of boundary layer thicknesses such that they are resolved by one or two gridpoints is a minimum thickness. Increasing the value of the friction $r$ widens the boundary layer and increases resolution. No new behaviour was found for higher boundary layer resolution obtained in this manner, nor for the case in which friction is held fixed and the number of gridpoints increased (Salmon, personal communication). If friction is reduced so that the boundary layer is thinner than one gridpoint, however, large discontinous changes and eventual unbounded growth occurs.

The other western boundary layer in the model is a diffusive boundary layer, with thickness $\frac{K_{H} f^{2} m^{2} \pi^{2}}{\beta N^{2} H^{2}}$, where $m$ is the vertical mode number and $H$ is the total depth. In the regime in which the diffusive layer is thicker than the Stommel layer, the diffusive boundary layer brings the incident normal flow to zero and can carry an $O(1)$ vertical transport, and hence closes the internal flow generated by heating. The dominant physical balances for this layer are geostrophic $\left(\beta v=f w_{z}\right)$ 
and hydrostatic $\left(\phi_{z}=g \alpha \theta\right)$, with upwelling or downwelling balancing horizontal temperature diffusion $\left(w \bar{\theta}_{z}=K_{H} \theta_{x x}\right)$. Inserting typical scales $K_{H}=2 \times 10^{6} \mathrm{~cm}^{2} \mathrm{~s}^{-1}$, $f=10^{-4} \mathrm{~s}^{-1}, m=1, \beta=10^{-13} \mathrm{~cm}^{-1} \mathrm{~s}^{-1}, H=4 \times 10^{5} \mathrm{~cm}, N=10^{-3} \mathrm{~s}^{-1}$, the thickness of the diffusive layer is about $200 \mathrm{~km}$, or one grid spacing. For the runs presented here the scale appeared to be somewhat wider, about 2-3 gridpoints (Figure 4.1). The Stommel boundary layers, in comparison, were one gridpoint wide on the western boundary and showed the expected widening from east to west on the northern boundary (Figure 4.3, upper panel). The requirement that the diffusive boundary layer be resolved by at least one gridpoint constrains the diffusion coefficient $K_{H}$. In practice $K_{H}$ was simply fixed at $2 \times 10^{6} \mathrm{~cm}^{2} / \mathrm{s}$, which gave adequate resolution without being too diffusive in the interior. Vertical temperature diffusion was also constant at $1 \mathrm{~cm}^{2} / \mathrm{s}$.

In the analytical model the strength of the heat source is measured by isotherm deviation $(\epsilon / \gamma)$. The connection between heating and isotherm deviation depends on the stratification. In the numerical model $Q / N^{2}$, where $\mathrm{N}$ is the buoyancy frequency, is analogous to $\epsilon / \gamma$, and represents the vertical velocity at the source. So a weak heat source can have a large effect if the stratification is also weak. Together with latitude, $\mathrm{N}$ determines the phase speed of waves in the model, and thus a weakly stratified layer at high latitude has the best chance for an important nonlinear effect, as measured by the condition that the phase speed be comparable to the particle speed. In the solutions presented, $N$ is either $7 \times 10^{-3} \mathrm{~s}^{-1}$ or $10^{-3} \mathrm{~s}^{-1}$, and the central latitude of the basin is either $25^{\circ} \mathrm{N}$ or $40^{\circ} \mathrm{N}$ (the basin extends $20^{\circ}$ north and south of the central latitude). The phase speed of the lowest and fastest mode is $2.7 \mathrm{~cm} / \mathrm{s}\left(25^{\circ} \mathrm{N}\right)$ or $0.27 \mathrm{~cm} / \mathrm{s}\left(40^{\circ} \mathrm{N}\right)$ at the central latitude. This condition for a nonlinear effect is observable, with particle speeds given by the shear flow, and the indication from data is that it could be important, even in the interior. In contrast, vertical advection is always dominantly linear, because the temperature boundary condition sets a vertical temperature gradient which makes the isotherms basically flat everywhere.

In order to compare numerical and analytical solutions a background flow whose forcing mechanism is unrelated to heating is required. This flow is created by extending the stress divergence term throughout the layer, that is by applying a body force to the entire water column. The depth-dependence of this body force can be varied, giving complete control over the background flow structure. The horizontal pattern of this flow was chosen in most runs to mimic the Stommel and Arons (1960) horizontal flow pattern (see Figure 4.3). Thus the background flow is to the north and east in the interior, away from the western and northern boundaries. Such a pattern of flow does occur in the abyssal layer of this numerical 
model's solutions of the general circulation (Salmon, personal communication). The background vertical velocity is zero in those runs for which the applied body force $\tau_{z}$ is independent of depth. In this case there is no background vertical shear and therefore no projection of the wind forcing onto internal modes, so the flow closes horizontally in northern and western boundary layers. Otherwise internal modes are forced by vertical structure of the stress divergence and they compete with those forced by the heat source $Q$.

The equations were time-stepped at intervals ranging from 10 days to 500 days, depending on the maximum velocity in the basin. A typical step was 1 year in the runs with weaker stratification. A steady solution always existed up to the point when $\mathrm{Q}$ was so large that the basic stratification broke down becoming unstable. Typically the total integration time was that required for mode 4 to cross the basin. Longer runs were done occasionally as a check. This study took altogether about one hour of Cray time at the San Diego Supercomputer Center.

\subsection{Response}

In this section solutions are presented with different heat source strengths and background flow strengths. To begin the discussion of the numerical solutions the simplest case will be presented: a single forced internal mode and no background flow. In these examples only the lowest mode is forced $\left(\mathrm{Q}^{1}=1, \mathrm{Q}^{2}=\mathrm{Q}^{3}=0\right)$. In one case the heating is weak, and in the other it is strong (Figures 4.1 and 4.2).

It is helpful to define some quantities to characterize the overall behavior of a solution. A useful measure of the nonlinearity of the solutions is the ratio of the horizontal advection of temperature to the vertical advection of temperature (HA/VA). This number is of the same order as $\epsilon / \gamma$. When this ratio is $\mathrm{O}(1)$ the basic stable stratification can be destroyed because then the perturbation temperature is essentially the same size as the background vertical variation. That is, $\frac{u \cdot \nabla \theta}{w \bar{\theta}_{x}}=\frac{a \theta}{L \bar{\theta}}$ since $\mathrm{u}=\mathrm{O}(a w / H)$ from the large-scale vorticity equation (equation 6 without the friction term), where $a$ is earth's radius, $H$ and $L$ are length and height scales respectively. This limit represents the extreme nonlinear case. The other quantity is the temperature Peclet number $\left(P_{e}\right)$, which in this chapter is the ratio of horizontal advection to horizontal diffusion. It is a measure of the importance of horizontal diffusion. These quantities are calculated as root mean square values over the entire basin.

Vertical diffusion was always smaller than horizontal diffusion in the runs shown here. It is still important for high modes, however, despite being small in the overall balance. Some runs (not shown) were made with strong vertical diffusion compared to horizontal diffusion, which had the effect of trapping the variations to the source. 
In these runs the vertical diffusion was several times greater than the canonical value of $1 \mathrm{~cm}^{2} / \mathrm{s}$. It did not prove possible to examine the case of "typical" or weak vertical diffusion combined with relatively small horizontal diffusion, as was the case in the analytical model, because of numerical convergence constraints. Weaker horizontal diffusion simply gives rise to stronger gradients. The analytic model; however, was for the interior region only, and did not include solutions for the flow immediately surrounding the source where horizontal diffusion is expected to be important. A heat source which was less localized might allow vertical diffusion to be relatively more important, but it also would not be as realistic.

At the source, a strong upwelling is generated by the heating (Figure 4.1). The balance $w \overline{\theta_{z}}=Q$, where $\theta$ has been replaced by the background profile, gives the right scale for $w$, even though friction and diffusion are significant. The nonhydrostatic term in equation $3(r w)$ is always of secondary importance to buoyancy and the vertical pressure force. The upwelling stretches the levels below mid-depth and compresses the levels above, inducing meridional flow and relative vorticity consistent with the vorticity equation. The stronger isotherm deviation towards the pole results from the variation of $f$, since stretching is more effective at high latitude. The effect of the heating decays to the east of the source in one or two gridpoints which is the approximate width of the diffusive and frictional boundary layers. To the west of the source the water downwells in a broad region extending to the boundary. In the middle left panel of the figure the downwelling is somewhat hard to see because of the large magnitudes at the source, but it is more apparent in the lower right meridional section. The temperature field extends westward from the source across the basin and sharpens in a western boundary layer. Some weaker flow exists outside the region of isotherm deviation, especially near corners, but this flow is ignored.

In this run the nonlinear effects are negligible. The ratio of horizontal advection and vertical advection is $10^{-5}$. Horizontal diffusion is important, as indicated by the small Peclet number, $\mathrm{Pe}=10^{-4}$. In this case the heat balance in the interior region west of the source is that downwelling heats deep levels and is balanced by diffusion.

When only a single mode is present, the thermal wind implies that the pressure field is parallel to the temperature field. This situation clearly holds in Figure 4.1, where the heating is weak. In figure 4.2 this is no longer true because other modes have been generated by nonlinear interaction. The ratio of horizontal and vertical advection is much larger, $10^{-1}$. Another indication of nonlinearity is that although $\mathrm{Q}_{s}$ increased by a factor of $10^{4}, \mathrm{Wmax}$ increased by only $10^{3}$. Since only a single mode is forced, it might seem puzzling that there is any nonlinear interaction. However the flow is not purely geostrophic. The frictional component gives rise to 

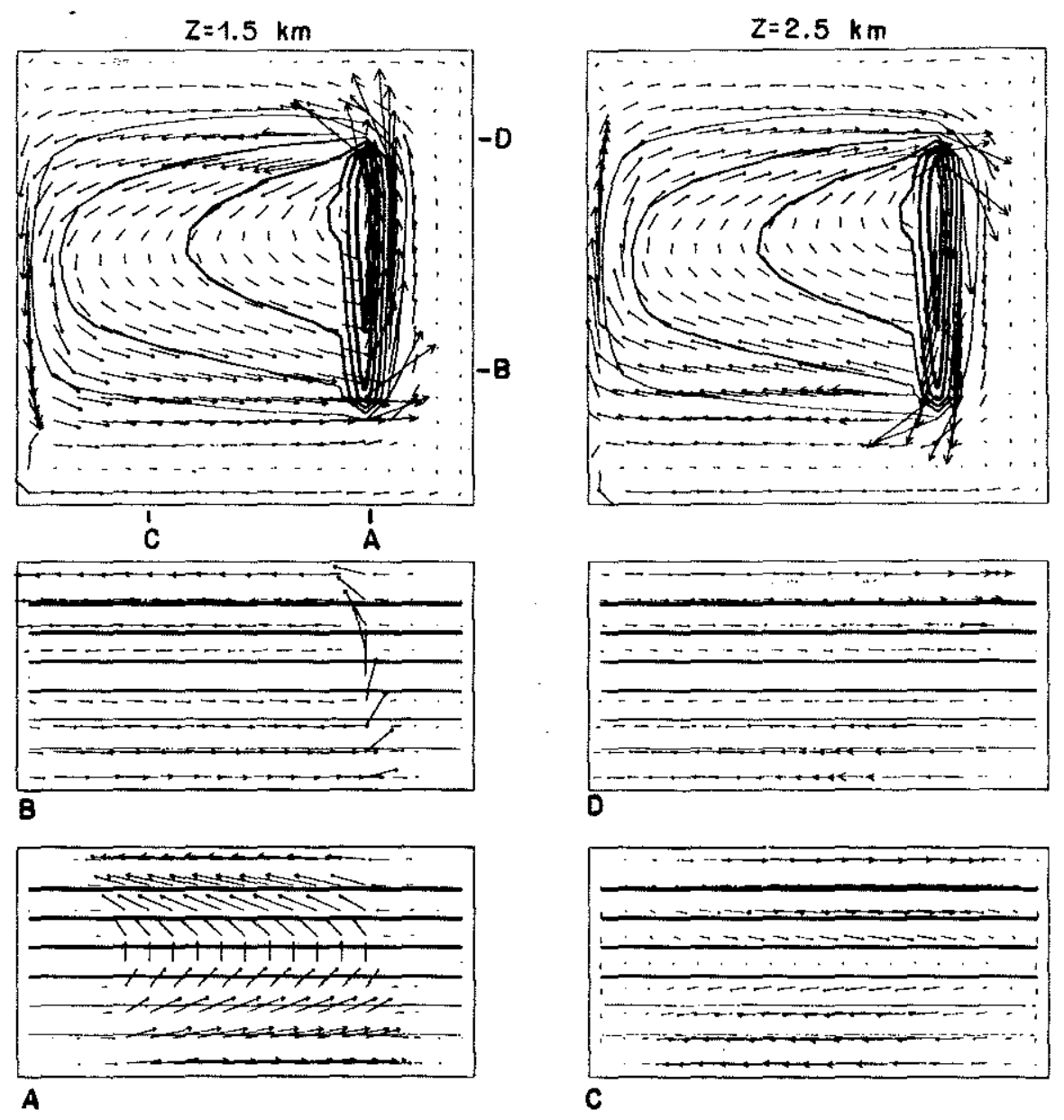

Figure 4.1: Single forced mode solution, no background flow. The upper two panels are plan views of temperature and velocity at $z=1.5 \mathrm{~km}$ above the bottom (left) and $2.5 \mathrm{~km}$ above the bottom (right). Below these panels are four sections, two zonal (middle pair), and two meridional (lower pair), of temperature and velocity. These levels and sections are the same in following illustrations of solutions. The source extends from $30^{\circ} \mathrm{N}$ to $50^{\circ} \mathrm{N}$ along a meridian $1000 \mathrm{~km}$ distance from the eastern boundary, and the source strength $\mathrm{Q}_{s}=0.01$. The central latitude is $40^{\circ} \mathrm{N}$, and $\mathrm{N}=7 \times 10^{-3} \mathrm{~s}^{-1}$. The maximum horizontal speed is $U \max =3 \times 10^{-4} \mathrm{~cm} / \mathrm{s}$, $\mathrm{W} \max =1 \times 10^{-6} \mathrm{~cm} / \mathrm{s} . \mathrm{HA} / \mathrm{VA}=10^{-5}, \mathrm{Pe}=10^{-4}$. 

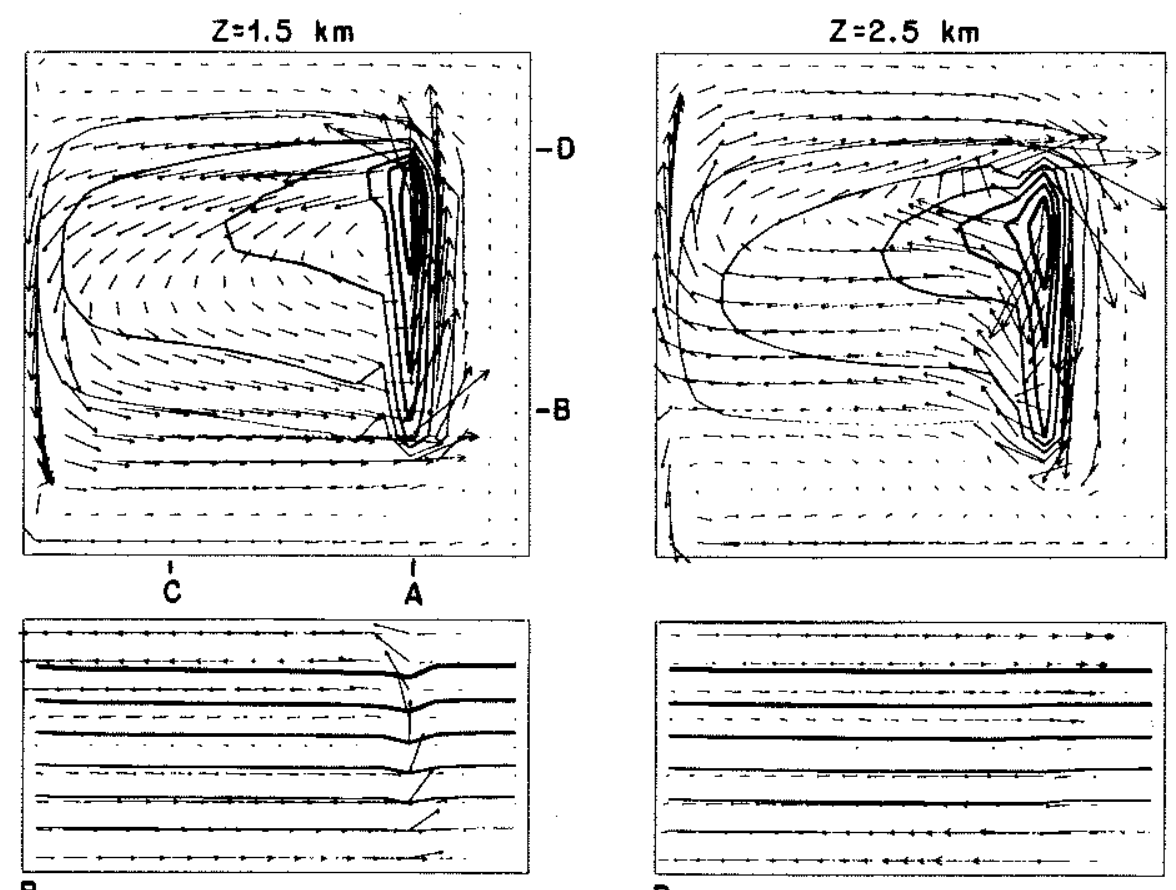

B
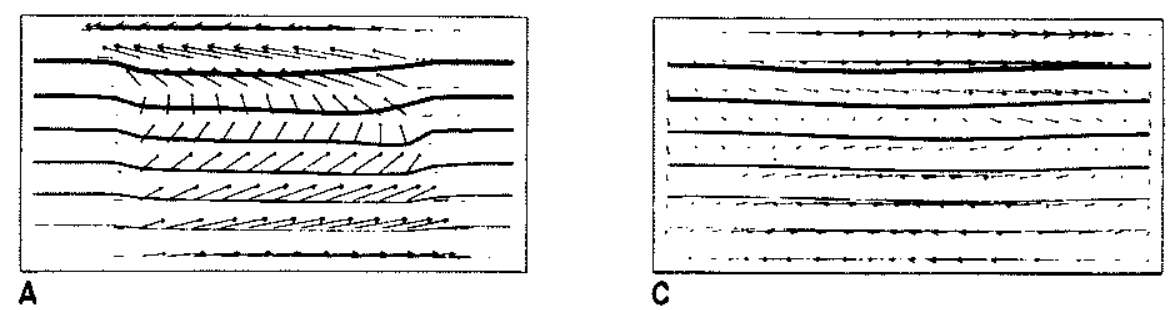

Figure 4.2: Same geometry as 4.1, $\mathrm{Q}_{s}=100 . \mathrm{U} \max =3 \mathrm{~cm} / \mathrm{s}, \mathrm{Wmax}=1 \times 10^{-3} \mathrm{~cm} / \mathrm{s}$. $\mathrm{HA} / \mathrm{VA}=10^{-1}, \mathrm{Pe}=1$. 
a small self-interaction, of order $r a^{2}$, where $a$ is the amplitude of the flow. In modal form,

$$
u \theta_{x}+v \theta_{y}=-\frac{r \pi}{2 H}\left[\left(u^{1}\right)^{2}+\left(v^{2}\right)^{2}\right] \sin (2 \pi z)+O\left(r^{2}\right)
$$

Physically, friction allows flow down the pressure gradient and hence across isotherms. Heating or cooling occurs because the flow tries to preserve its temperature. Thus in the lower, cyclonic (low pressure) part of the solution, inflow cools the source requiring a negative mode 2 amplitude to partially cancel mode 1 there. The net effect is like a small upward shift of the heat source. This interaction is weak, so it is necessary to compare its strength to the weak nonlinear effect from the vertical advection term. This term is

$$
w^{\prime} \theta_{z}^{\prime}=\frac{m w^{1} \theta^{1}}{2} \sin (2 \pi z)
$$

where

$$
w^{1} \theta^{1}=\frac{g \alpha\left(\theta^{1}\right)_{x}^{2}}{2 \rho f^{2} m^{2}}
$$

to lowest order in $r$. Vertical advection changes sign across the source as $w$ changes sign from downwelling to upwelling. The downwelling of a mode 1 temperature structure heats water below mid-depth $\left(w \theta_{z}^{\prime}>0\right)$ and cools water above mid-depth $\left(w \theta_{z}^{\prime}<0\right)$ while upwelling does the opposite. Thus a positive mode 2 is forced west of the source and a negative mode 2 is forced east of the source. For a single mode the ratio of horizontal to vertical advection is $\frac{r u^{2}}{w \theta}$, which is $O(1)$ for the runs shown (in the units of the model $r^{-1}=10$ days, $\theta=10^{3} \mathrm{~km} /$ day $^{2}$, and $\bar{\theta}=4 \times 10^{5} \mathrm{~km} /$ day $\left.^{2}\right)$. Thus both terms can give rise to higher modes from a single forced mode. For the solutions shown here, though, the structure of mode 2 for increasing $Q_{s}$ was found to be dominated by the ageostrophic generation term 4.8 (mode amplitudes from a series of runs not shown). The effects are easy to separate since the vertical advection term changes sign zonally across the source. At the source, horizontal advection dominates, generating negative mode 2 amplitude. Somewhat west of the source, the horizontal advection term is still significant. Vertical advection has a small, secondary effect, generating positive mode 2 amplitude west of the source and negative amplitude east of the source. This indicates that the ageostrophic advection of heat is important for generating higher modes from a single forced mode. Once mode 2 is generated, stronger interactions between different modes can take place. These interactions take place mostly near the source, because the amplitude of the flow is greatest there. It might be anticipated that when more than one mode is forced the nonlinear terms become important for smaller $Q_{s}$, and this was found to be true in other runs. 
Many of the same remarks made about the weakly forced case apply to the strong heating case. The vorticity balance (which is always linear) connects upwelling and downwelling to meridional flow in the interior. The pattern of the solution has changed significantly. While the same broad sense of circulation exists with clockwise flow in the upper layer and counterclockwiseflow in the lower layer, the pattern is different, and especially in the upper layer, the strong part of the flow has constricted to about $\frac{1}{4}$ of the meridional extent it had when $\mathrm{Q}_{s}=0.1$. Likewise the isotherms have constricted meridionally giving the appearance of coming from a much more localized source. Curiously, together with the velocity, the temperature looks like a passive tracer downstream of a source. This contrasts to the weakly heated case, where velocity was parallel to isotherms not because horizontal advection was important, but by accident of vertical structure (in this example the case of a single mode; the sum of multiple forced modes result in isotherms and isobars which are not necessarily parallel).

The Peclet number is small in the linear example because horizontal advection is tiny. In the nonlinear run horizontal advection and diffusion are of equal importance, and the interior heat balance is made up of three terms. Heating caused by downwelling warm water and by horizontal advection from the warm source region is balanced by diffusion.

Throughout the interior core of the warm plume emanating from the heat source, both vertical and horizontal advection are a local source of heat. In this example, the only way to get rid of this heat is through horizontal diffusion. Therefore the plume must constrict to such a scale that horizontal diffusion can act. The $\beta$ effect keeps the zonal scale large, and so the plume becomes narrow. Balancing these two terms to find the appropriate meridional scale gives $\mathrm{y}=\left(\mathrm{K}_{H} L / U\right)^{\frac{1}{2}}$, which is $400 \mathrm{~km}$ for $L=4000 \mathrm{~km}, \mathrm{~K}_{H}=2 \times 10^{6} \mathrm{~cm}^{2} / \mathrm{s}$ and an $\mathrm{rms} U=0.5 \mathrm{~cm} / \mathrm{s}$. A smaller velocity scale not biased by large boundary layer values should probably be used, and this would increase the scale somewhat. However the number seem to be about right for the nonlinear plume and probably explains its narrowness.

The preceeding remarks are general and illustrate the entire range of nonlinear behavior found in all the solutions. Several examples are given next to make a comparison between the numerical and analytical models, and a series of runs are shown to illustrate the modal response of the system to increasing heating and stronger background flow. 


\subsubsection{An example of mode separation and nonlinear correc- tion field.}

A basic feature of the analytic solutions is the different characteristic curves for different modes, that is, the tendency for modes to separate. This happens because of the variation in phase speed of the modes with mode number and latitude, which means that a given background flow will advect high modes more than low modes, and that a given mode will have a greater westward influence at low latitudes. The overall controlling parameter, $\mathrm{C}$, was found in Chapter 3 to be the ratio of the phase speed and the background flow speed.

One of the primary goals was to look for this behavior in the numerical model, to see if the concept was relevant or not in a more complicated system. To illustrate the effect of increasing the background flow strength (decreasing $C$ ) on a given mode, the amplitude of mode 2 from the numerical model for three different values of $\mathrm{C}$ is shown (Figure 4.3). To evaluate $\mathrm{C}$ the phase speed at the central latitude $\left(40^{\circ} \mathrm{N}\right)$ has been used. For $\mathrm{C}$ equal to infinity the influence is purely westward. When $C$ decreases to 1 , mode 2 is held near the source, and when $C$ gets to be 0.5 the influence is eastward or downstream. At this point the influence is almost purely passive. The amplitude of the mode changes by no more than about $10 \%$ over this range, and only by $1 \%$ from $\mathrm{C}=\infty$ to $\mathrm{C}=1$. The flow pattern (shown for $\mathrm{C}=1$ ) changes gradually to that of the background flow alone at $\mathrm{C}=0.5$. There is some extension of the plume westward in the northern boundary current at low values of C.

The behavior of the solution at a fixed $C$ is shown to illustrate mode separation in the numerical model (Figure 4.4). In this case only three vertical modes were used, and all three are shown. The more passive behavior of the higher modes is apparent; and again they can be advected in boundary currents.

In either case of changing $\mathrm{C}$ or fixed $\mathrm{C}$, the response was as expected, for both the value and changes in $\mathrm{C}$. Furthermore this response was consistent and predictable throughout all the numerical solutions examined.

A correction field like that calculated analytically was made by subtracting the modal amplitudes of two runs, one nonlinear and the other linear (Figure 4.5). The forcing was the same $\left(\mathrm{Q}_{s}=50, \mathrm{Q}^{1}=0.51, \mathrm{Q}^{2}=0.0625, \mathrm{Q}^{3}=0.0255\right.$; note that these forced amplitudes are to be multiplied by $\mathrm{m}^{2}$ to give temperature mode amplitudes in agreement with the modal amplitudes chosen in the analytical model, since $m^{2} Q \sim \theta$ ) in both cases, but in the linear run the temperature equation (4.5) was linearized. This was done by replacing the full temperature by the background one in the vertical advection term, and by replacing the full horizontal velocity by the background one in the horizontal advection term. The strength of the forcing 


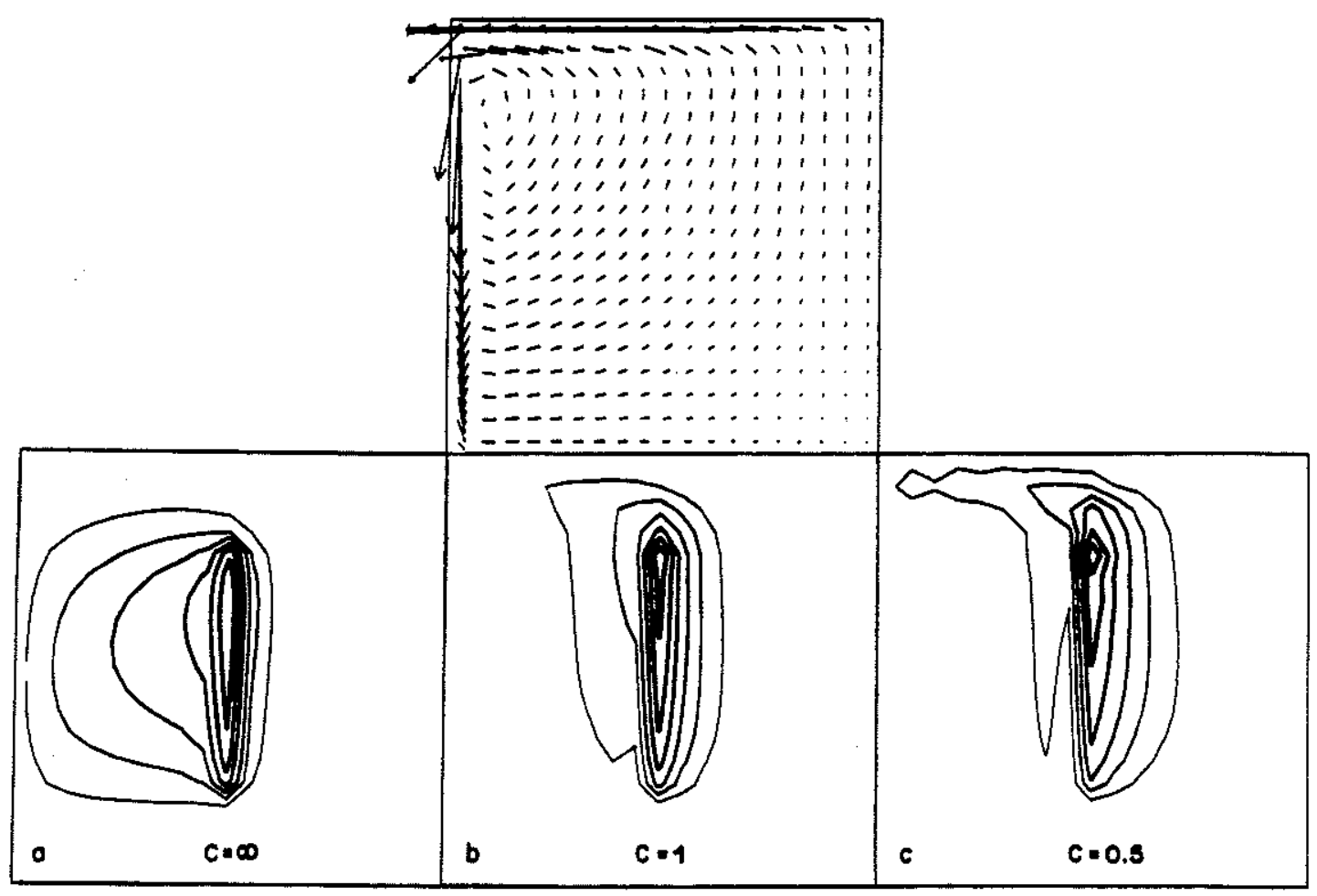

Figure 4.3: The horizontal structure of mode 2 for three values of $\mathrm{C}: \infty, 1,0.5$. The location of the heat source in this example is down the middle of the basin, and $Q_{S}=1, Q^{1}=0.5, Q^{2}=0.25, Q^{3}=0$. The background flow is shown in the solution at $z=1.5 \mathrm{~km}$, for $C=1$ (arrows, middle upper panel), and is northward and eastward in the interior. There is a northern boundary current with westward flow and a western boundary current with southward flow. 


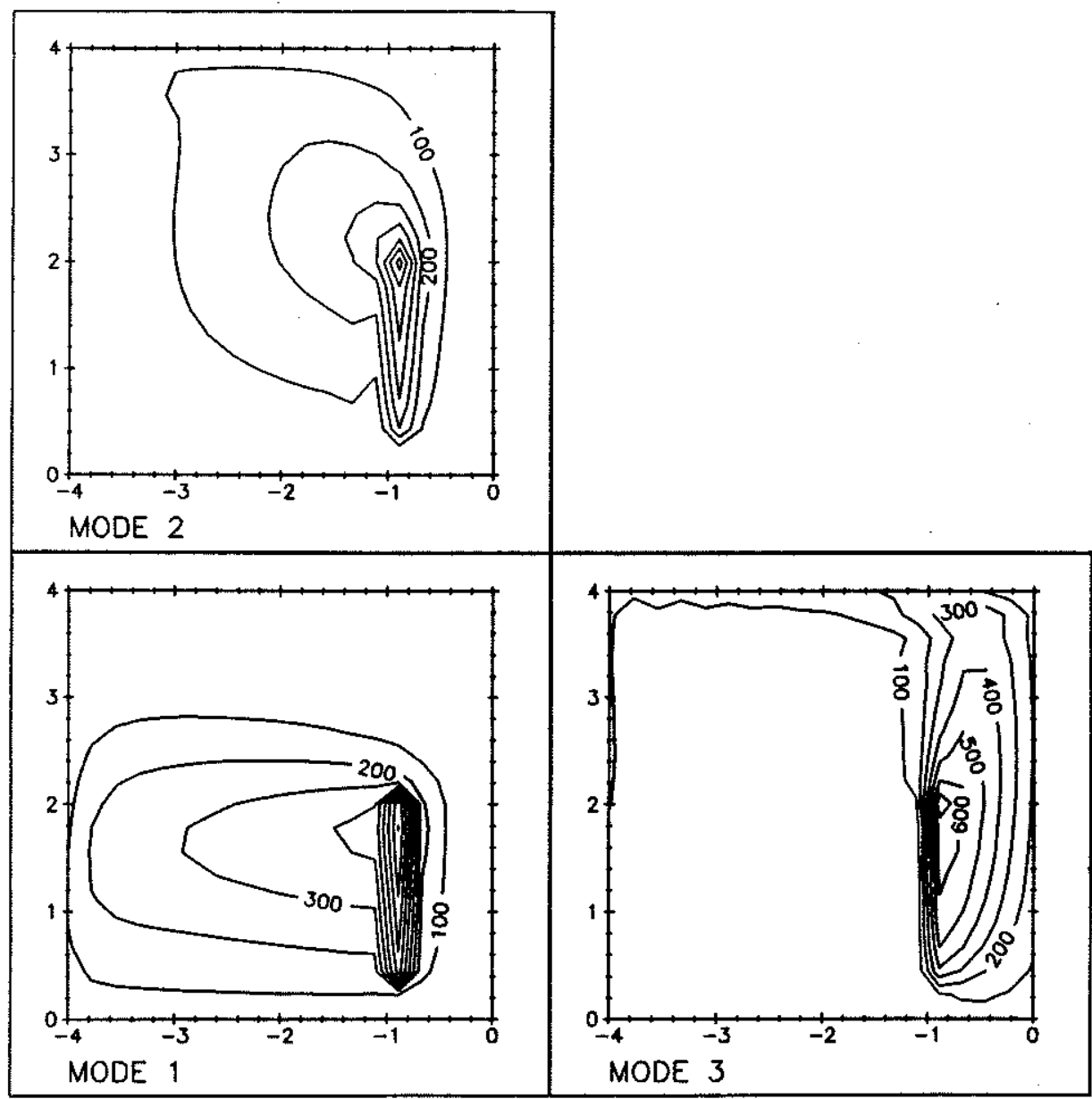

Figure 4.4: Mode amplitude showing separation for the case $\mathrm{C}=4$. The heating strength $Q_{s}=25, Q^{1}=0.5, Q^{2}=0.125, Q^{3}=0.055$. 


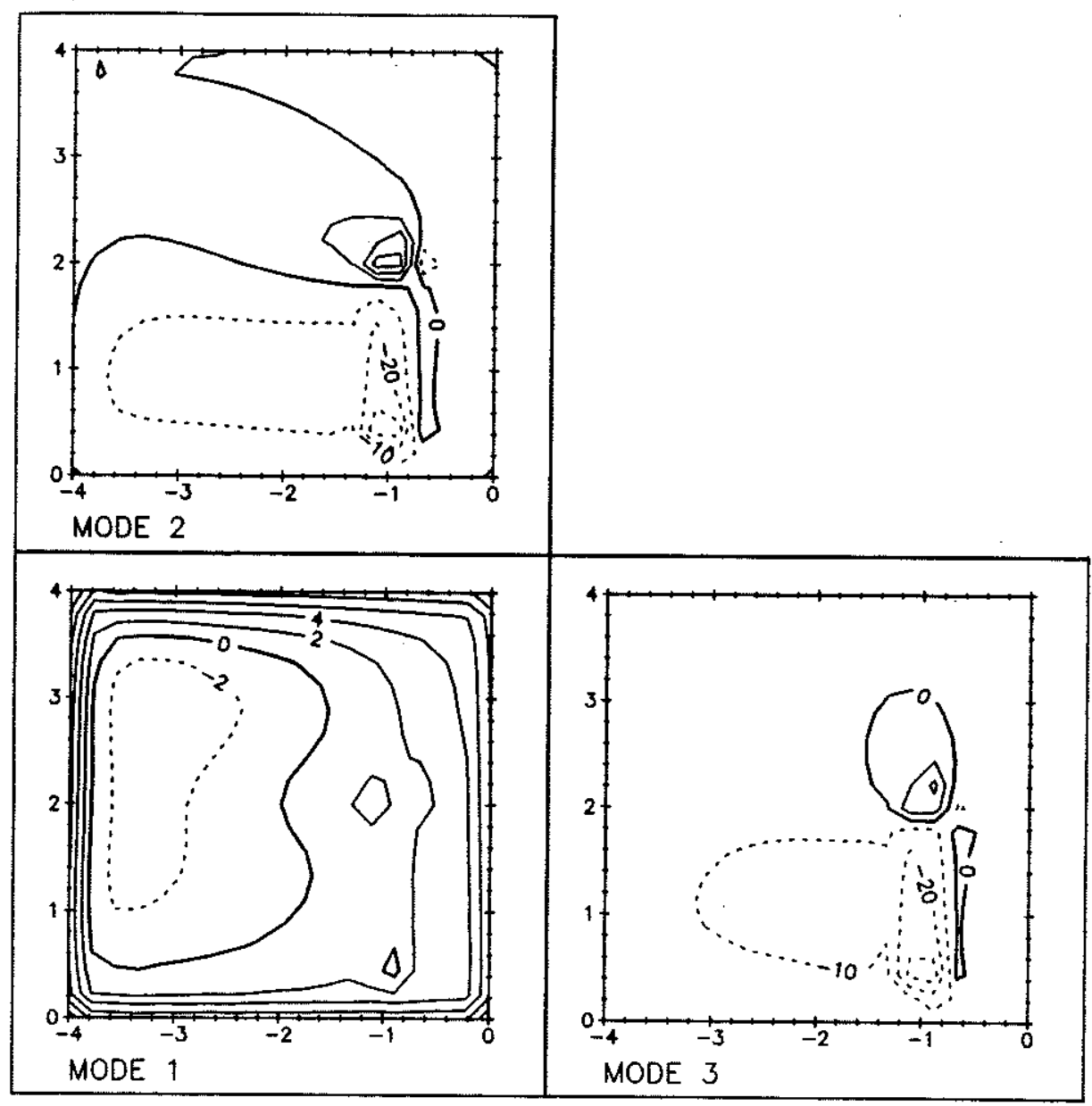

Figure 4.5: The correction field which must be added to the linear solution to get the nonlinear solution. The heating strength $\mathrm{Q}_{s}=50$, and $\mathrm{C}=1$. The maximum value of the mode amplitudes of the linear solution are mode $1=2400$; mode $2=$ 500 ; mode $3=250$. 
was chosen to correspond to the analytical case $a \epsilon / L \gamma=0.1$. The central latitudes is $25^{\circ} \mathrm{N}$, and $\mathrm{C}=1$. The Peclet number for vertical advection and diffusion is also 1. For this value of $\mathrm{C}$ the nonlinear solution looks much like the linear one. A qualitative difference arises only when $\mathrm{C} \gg 1$.

The mode 1 correction is negligible, less than $0.1 \%$. All that is visible is noise from background vertical structure which wasn't fully eliminated. The analytic correction for mode 1 was also very small, a few percent, with no important tendency (Figure 3.13). The tendency for modes 2 and 3 is to sharpen or steepen the linear patterns, as was found in the analytical case. The mode 2 correction is remarkably similar, but mode 3 is lacking as broad a region of positive correction as expected from the analytical model. The amplitude of the correction field is close to $10 \%$, which is correct for agreement since $a \epsilon / L \gamma=0.1$.

A series of runs is shown next to illustrate the pattern of behavior for increasing heating and background flow (Figures 4.6, 4.7, 4.8, 4.9). The relative mode amplitudes are as above, and the central latitude is $40^{\circ} \mathrm{N}$. The stratification has been reduced to $\mathrm{N}=10^{-3} \mathrm{~s}^{-1}$, which may better represent some regions of deep water. As a result nonlinearity will become significant at lower values of heating. Now the phase speed of the lowest mode is $.27 \mathrm{~cm} / \mathrm{s}$ at the central latitude. The first three have no background flow, and the last one has a background flow chosen to be comparable to interior velocities. Along with the temperature and velocity fields, the structure of modes 1 to 4 is shown for each run.

There is a smooth progression in the response of the solutions to increased heating. No sudden change in the character of the response was ever seen. A clear indication of stronger heating is the growing deviation of isotherms away from the flat basic state. For instance, a north-south variation in the vertical separation of isotherms (stability) is apparent in the strongly forced case (Figure 4.8). This variation exists west of the source to some degree (lower right panel).

In the case with background flow (Figure 4.9), the interior velocity field is substantially modified. However the mode amplitudes are virtually identical to the no background flow case with the same heating (Figure 4.7). Thus in spite of the significant nonlinearity of the solution $(\mathrm{HA} / \mathrm{VA}=0.1)$, it is essentially a superposition of the two separate flow fields. With $\mathrm{C}=22$ in the absence of heating, the interior horizontal velocity would be $0.01 \mathrm{~cm} / \mathrm{s}$, with a maximum of about $0.05 \mathrm{~cm} / \mathrm{s}$. This is comparable to the interior velocity of the gyre in the no background case, which is about an order of magnitude smaller than the maximum velocity, or about $.02 \mathrm{~cm} / \mathrm{s}$. Decreasing $\mathrm{C}$ to some lower value by increasing the background flow would eventually change the structure of the mode amplitudes as was shown before (Figure 4.3). Then the background flow would completely dominate the total velocity. Strong heating and strong background flow combine both mode separation effect and the 

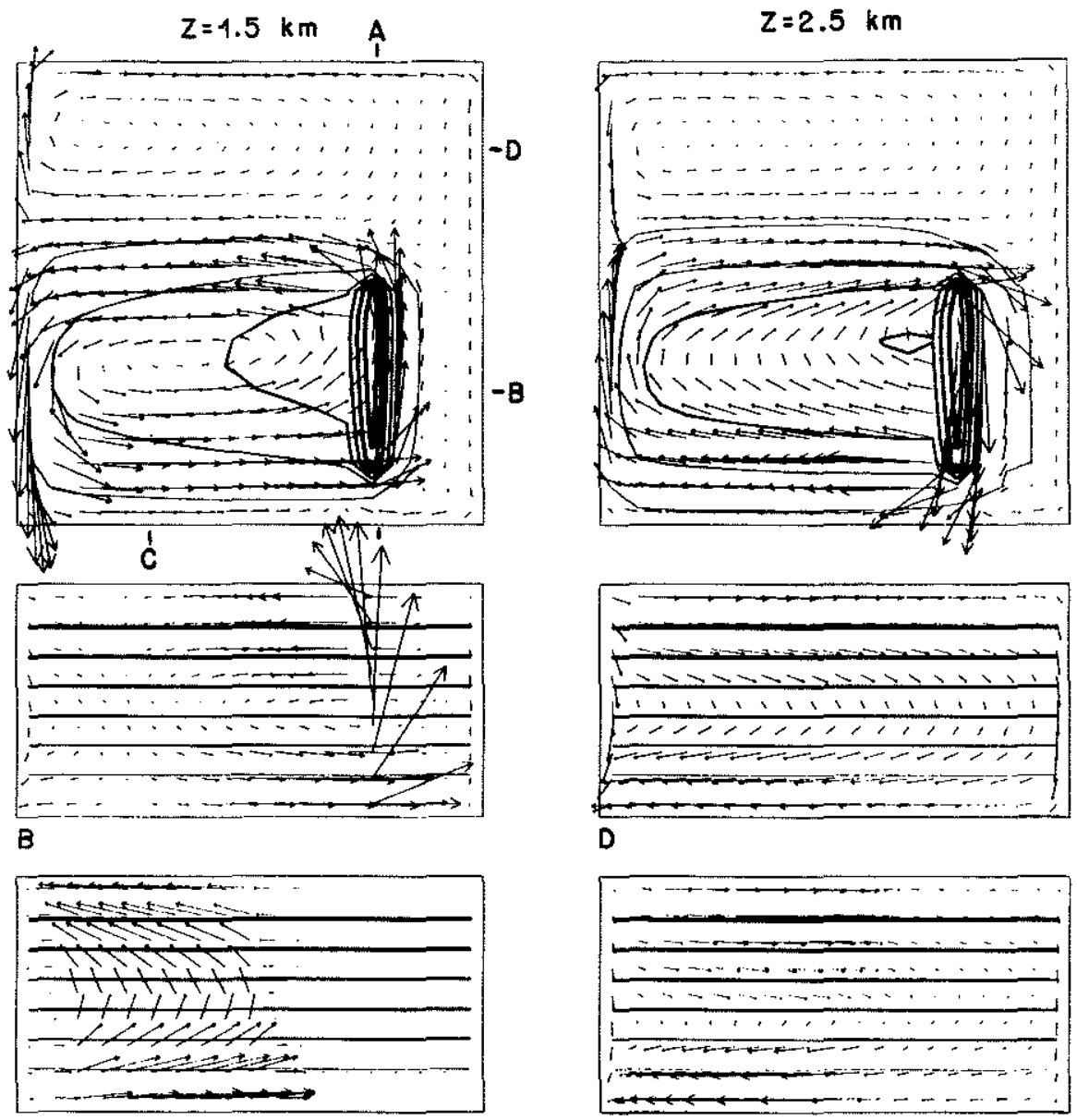

A

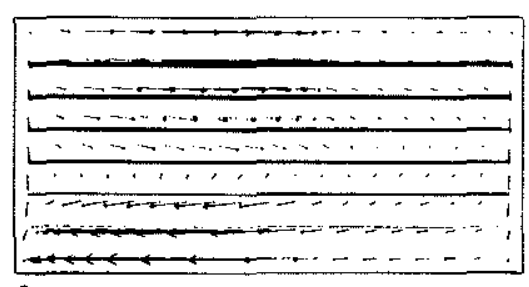

C

Figure 4.6: a) Solution for three mode forcing, $Q_{s}=0.1$, levels and sections are as in Figure 4.1. $\mathrm{N}=10^{-3} \mathrm{~s}^{-1}$, no background flow. The central latitude is $40^{\circ} \mathrm{N}$. The parameter $a \epsilon / L \gamma=3 \times 10^{-3}, U \max =.02 \mathrm{~cm} / \mathrm{s}, \mathrm{W} \max =10^{-5}, \mathrm{HA} / \mathrm{VA}=10^{-2}$, $\mathrm{Pe}=6 \times 10^{-2}$. 

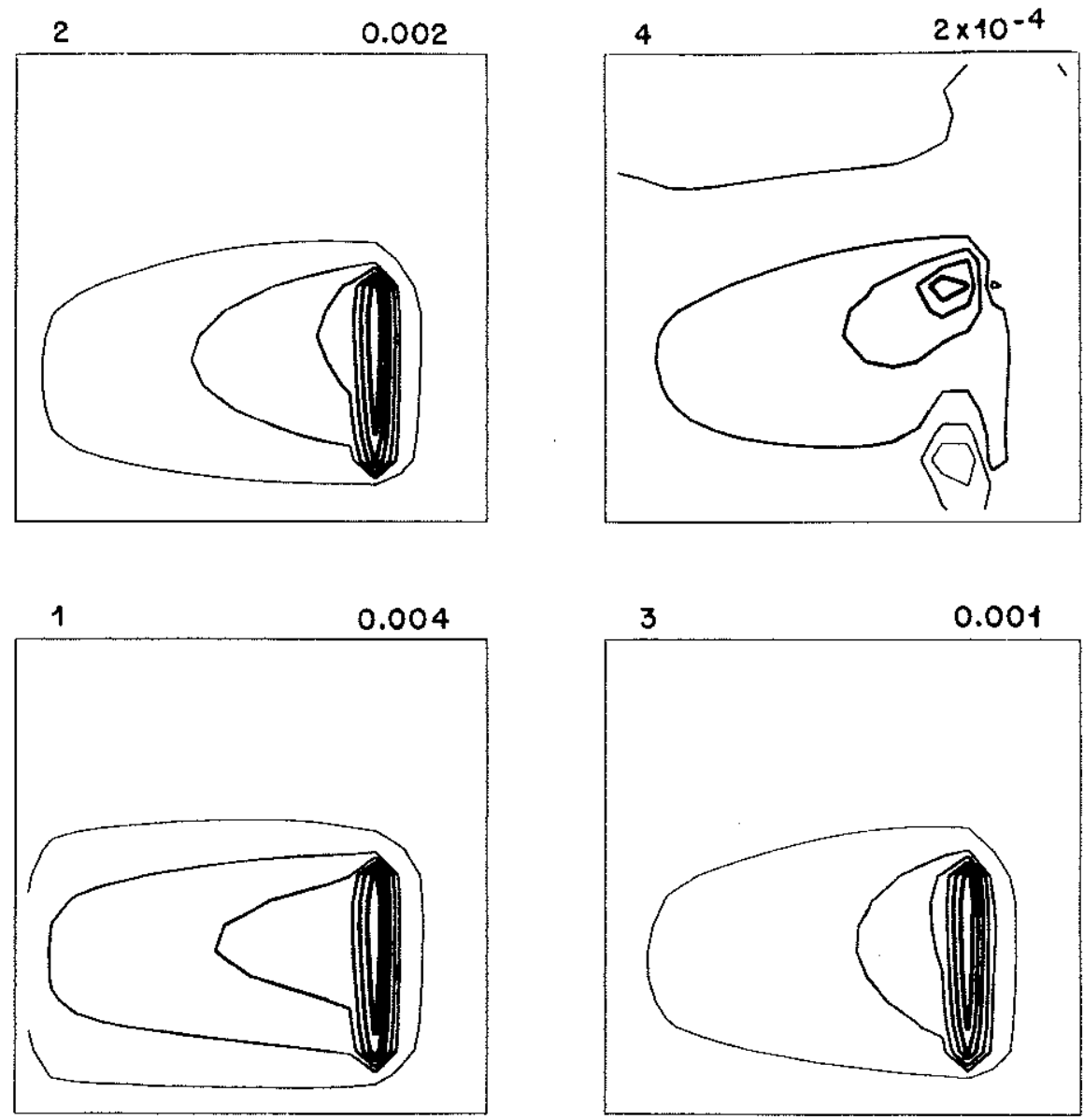

Figure 4.6: b) Mode amplitudes for $Q=0.1$, with maximum values. 

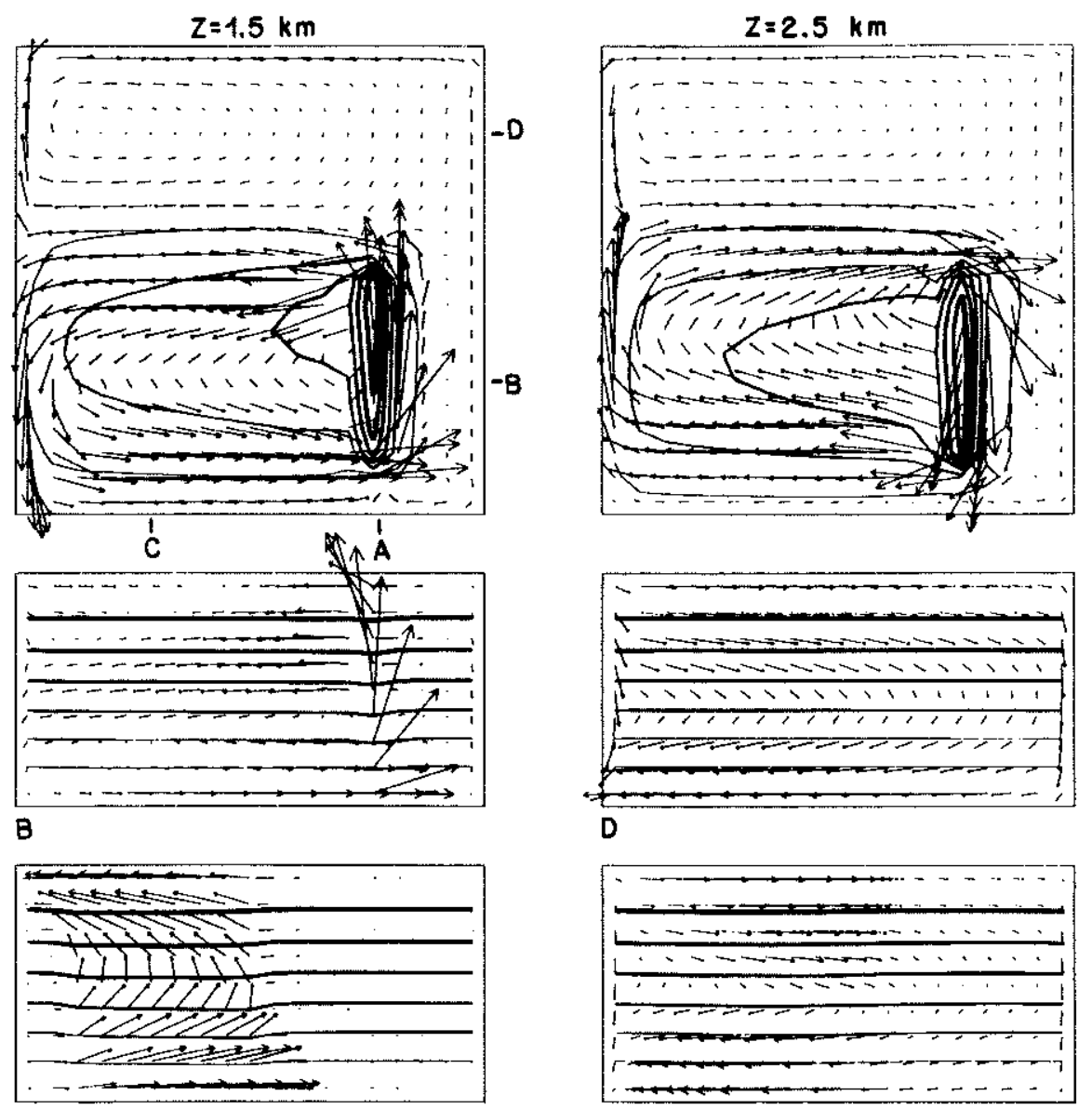

A

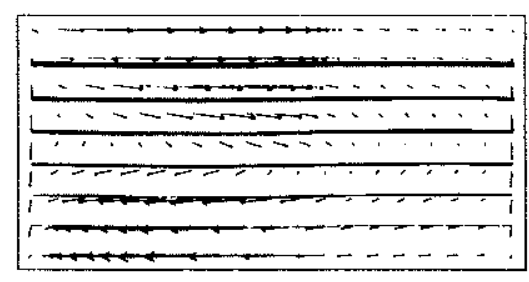

C

Figure 4.7: a) Same as 4.6 for $\mathrm{Q}_{s}=1$. The parameter $a \epsilon / L \gamma=3 \times 10^{-2}$, $\mathrm{U} \max =.2 \mathrm{~cm} / \mathrm{s}, \mathrm{Wmax}=10^{-4}, \mathrm{HA} / \mathrm{VA}=10^{-1}, \mathrm{Pe}=5 \times 10^{-1}$. 

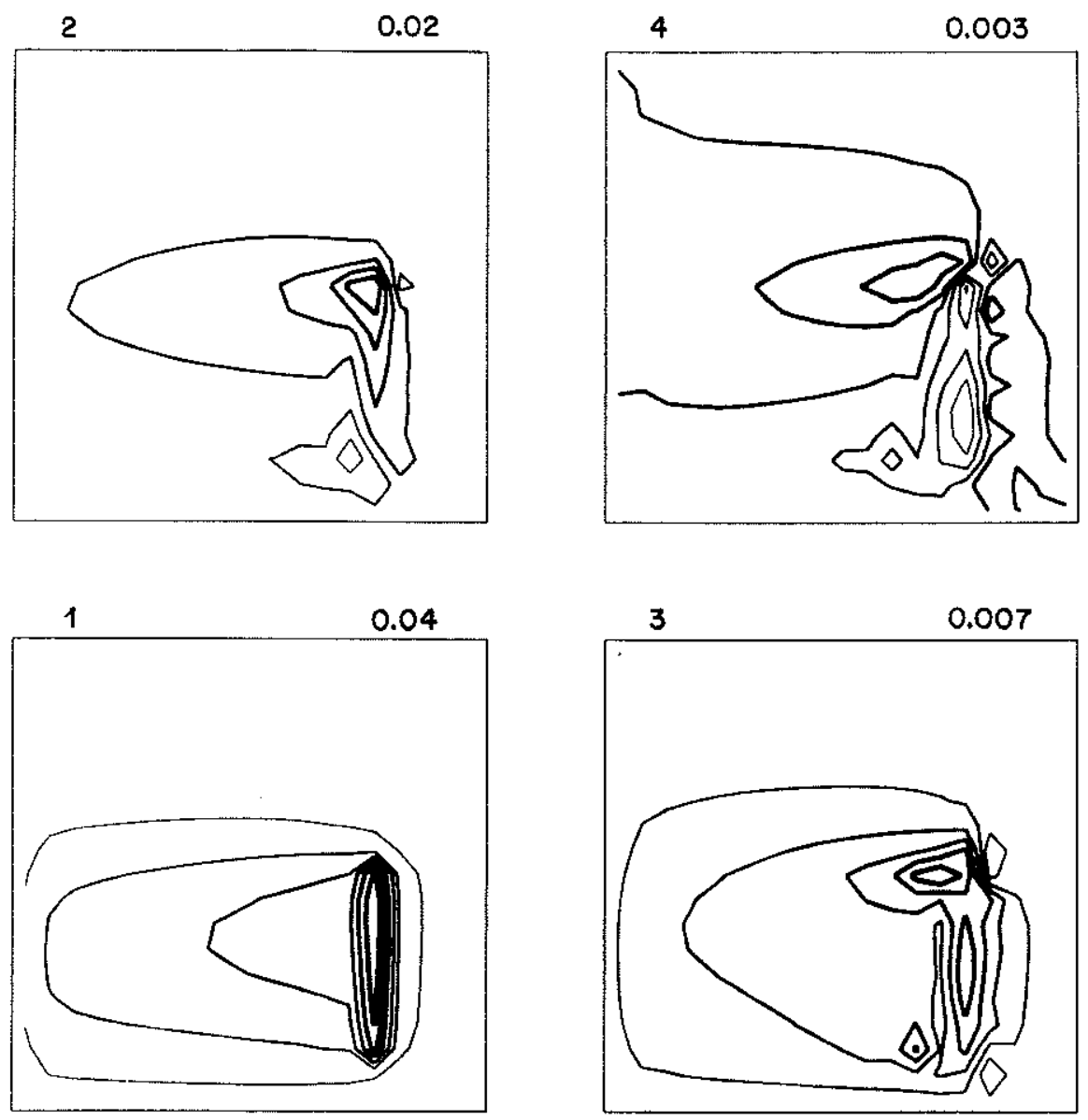

Figure 4.7: b) Mode amplitudes for $Q=1.0$, with maximum values. 

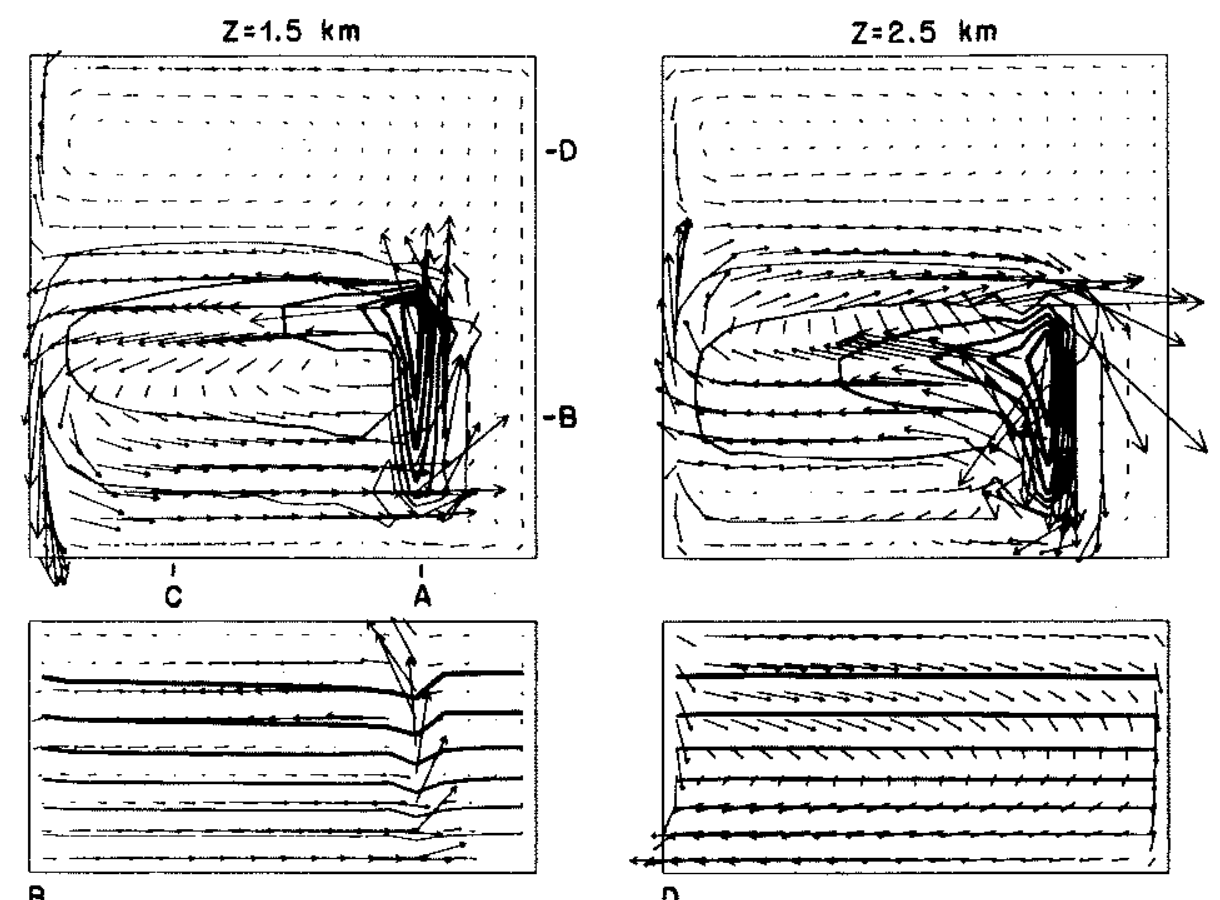

B

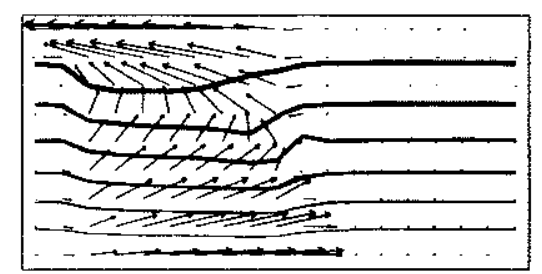

A

D

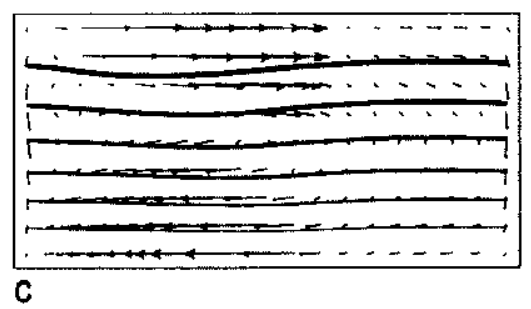

Figure 4.8: a) Same as 4.6 for $\mathrm{Q}_{s}=4$. The parameter $a \epsilon / L \gamma=0.1, \mathrm{Umax}=.6 \mathrm{~cm} / \mathrm{s}$, $\mathrm{W} \max =2 \times 10^{-4} . \mathrm{HA} / \mathrm{VA}=0.3, \mathrm{Pe}=2$. 

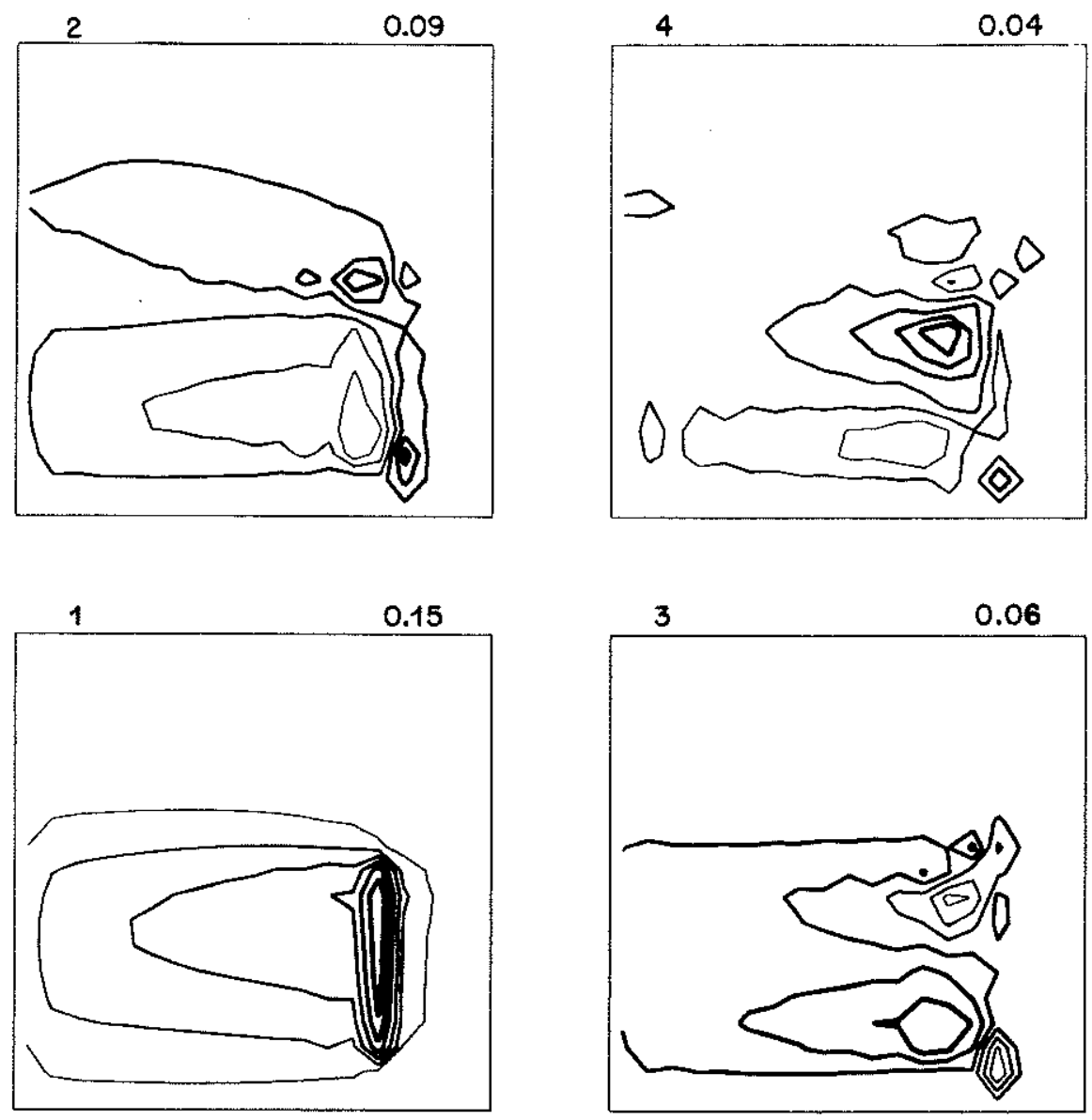

Figure 4.8: b) Mode amplitudes for $Q=4.0$, with maximum values. 

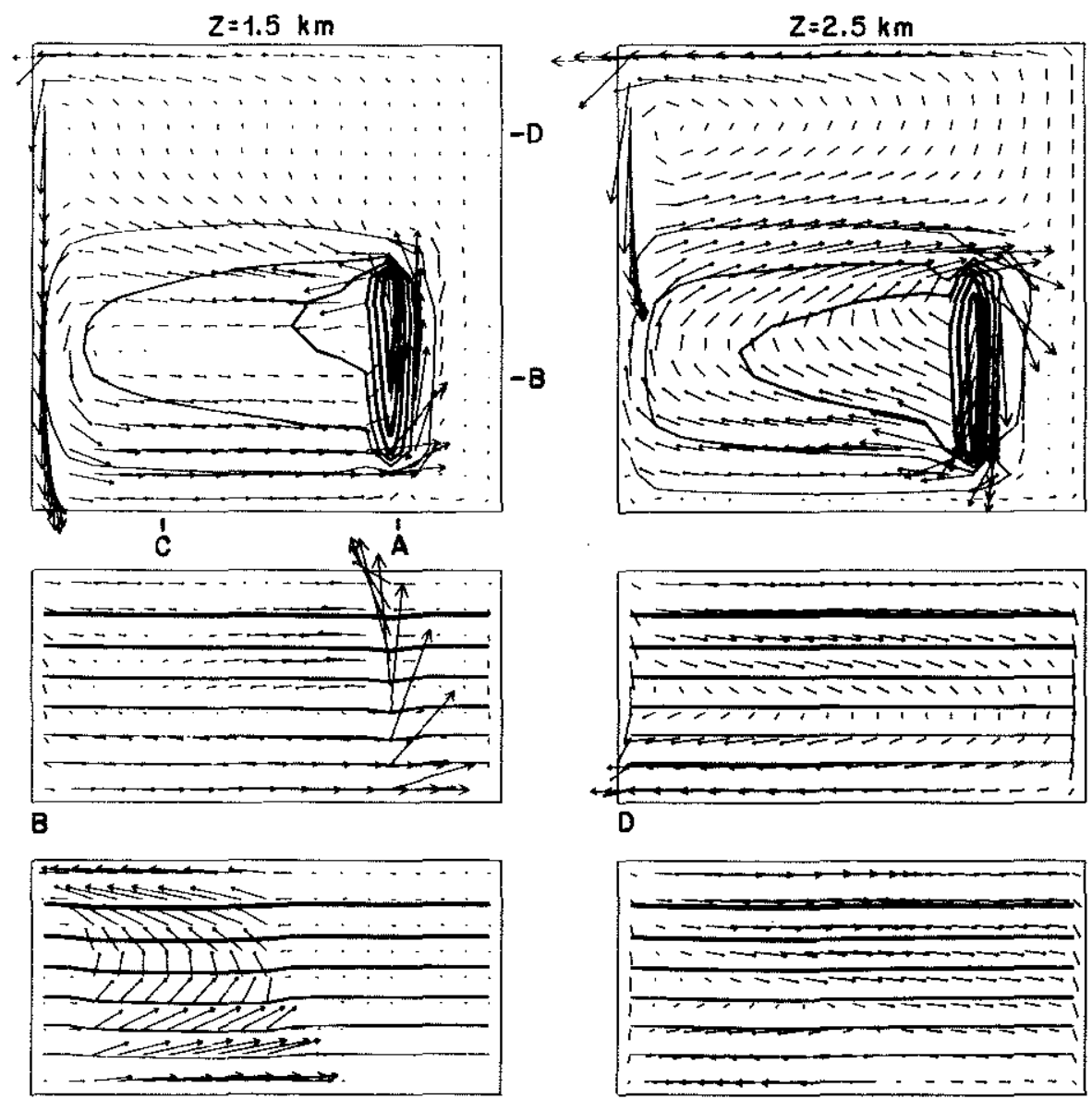

A

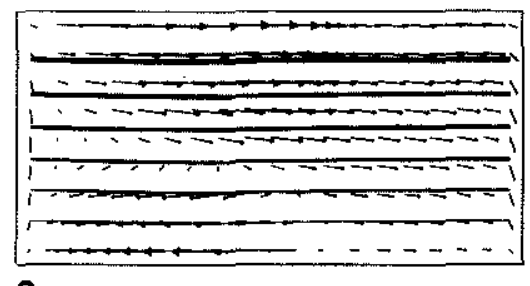

Figure 4.9: a) Solution with a background flow, $C=22$. Same geometry, heating $\left(Q_{s}=1\right)$, stratification, and parameters as 4.6 . 

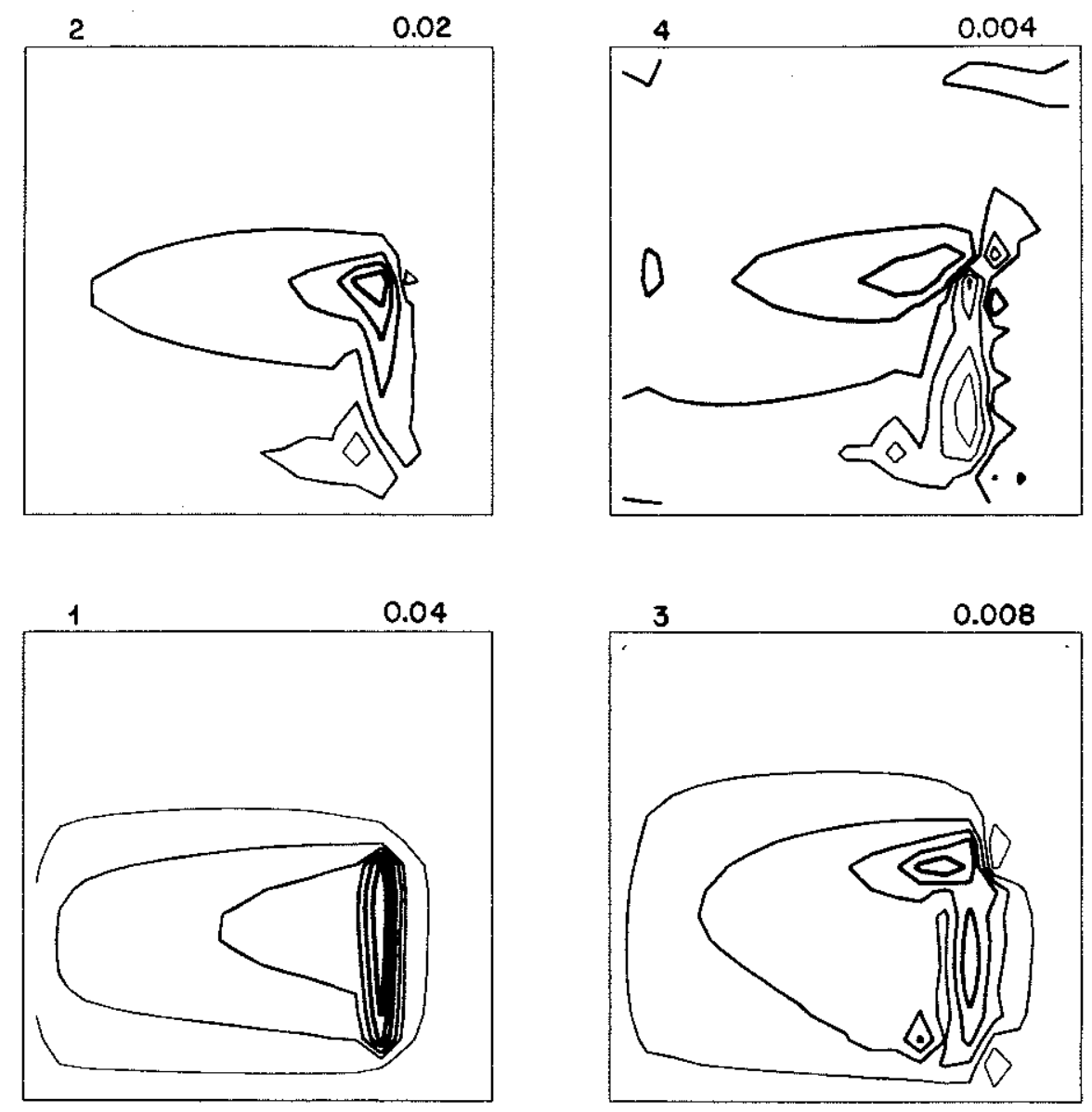

Figure 4.9: b) Mode amplitudes for case with background flow, and maximum values. 
modification of the background flow. As a result of the background flow a passive tracer can have a very different dispersal pattern compared to the position of the warm plume (Figure 4.9). A tracer injected in the upper layer at the source would spread west across most of the basin, but rather than returning directly east is carried into the poleward, eastern corner (upper right). Below mid-depth the tracer would be carried toward the poleward, western corner (upper left), before entering the southward flowing western boundary current.

Associated with the development of the narrow nonlinear plume is the gradually changing response of modes 2 and 3 . In figure $4.6 \mathrm{~b}$ the modal structure looks like that of the forcing. In figure $4.7 \mathrm{~b}$ the response is different, with an increasing negative component of mode 2 amplitude, beginning at the southern portion of the source. The mode 3 response is more complicated, but on the whole its relative amplitude has decreased at the source and increased in the interior (for all the modes, the magnitude of the response changes in proportion of the strength of the heating). In figure $4.8 \mathrm{~b}$ there is a strong negative component of mode 2 (lightweight lines). Mode 3 also changes sign along the source, but the sense of the variation is opposite mode 2. Mode 1 is essentially unchanged, except for the overall increase in magnitude.

This pattern arises because of horizontal heat advection, and can be interpreted from a modal point or view, or a physical space point of view. The modal interpretation is based on the nonlinear source term coming from the product of two or more modes, and written in terms of a Jacobian, which was used to calculate the correction field in the analytical model. The combination of dissipation and the $\beta$ effect produces a nested pattern of modes with higher modes inside lower ones. Thus mode 3 , for instance, decays to the west along contours of mode 1 amplitude. The Jacobian $\mathrm{J}\left(\theta^{3}, \theta^{1}\right)$ is then negative on the north side of the plume and positive on the south side, and so the nonlinear source term for mode 2 , which is proportional to $-\mathrm{J}\left(\theta^{3}, \theta^{1}\right)$, gives the observed tendency. The same arguement can be used to rationalize the changes in the structure of mode 3.

In physical space, the effect of nonlinearity is consistent with the idea of a local advection of temperature by the dominant flow pattern. For instance, the mode 1 flow twists temperature clockwise in the upper half of the layer and counterclockwise in the lower half. However it is more useful to examine the tendency of the temperature equation (5) to form a meridional gradient. Taking the y-derivative of the equation and neglecting the small vertical diffusion gives

$$
u \cdot \nabla \theta_{y}+u_{y} \theta_{x}+\overbrace{v_{y} \theta_{y}+w_{y} \theta_{z}}+w \theta_{z y}=\nabla^{2} \theta_{y} .
$$

The first term is small because the y-gradient near the source is small. It tends 
to reduce $\theta_{y}$ by advecting the small y-gradient near the source to the interior, and thus has the wrong tendency. The second term is small because of the large zonal scale of the plume, and the fifth term is small because the basic state is flat. The ratio of the two terms under the brace is $a \epsilon / \gamma \ell$, where $\ell$ is the meridional scale. This ratio is $0(1)$. In fact, because of the interior balance $\beta v=f w_{z}$, they are really the same effect (modally $\mathrm{v}_{y} \propto \mathrm{w}_{y}$ ). The term $v_{y} \theta_{y}$ is the convergence of $\theta_{y}$, while $\mathrm{w}_{y} \theta_{z}$ forces convergent meridional flow. In the steady state this tendency is balanced by meridional diffusion.

\subsubsection{Background shear}

The final complication is vertical shear in the background flow. The body force which generated the background flow of Figure 4.9 was multiplied by an exponential decay away from the top of the layer with a decay scale of $10 \mathrm{~km}$. As a result, the background horizontal velocity decreases downward approximately linearly to about $70 \%$ of its magnitude at the top, as shown below. This is meant to represent

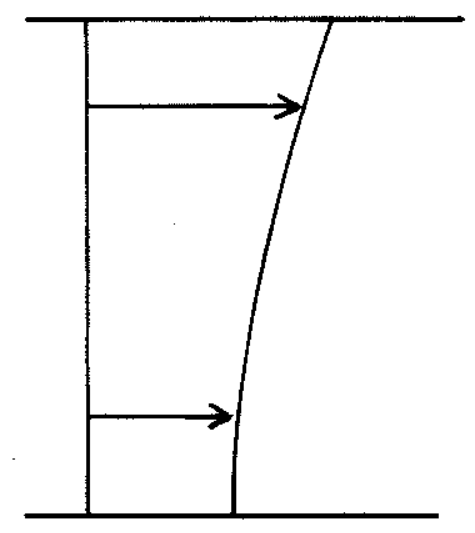

a deep shear profile below the thermocline, where the vertical scale of shear is probably larger than in the upper layer. The background vertical velocity $\bar{w}$ is also exponential, and this vertical structure projects onto internal modes, which compete with those generated by heating.

The resulting horizontal velocity field (Figure 4.10) is much the same as that without shear (Figure 4.9). The interaction of background shear modes with heatingforced modes creates sum and difference modal amplitude over a broad area. Direct forcing of an internal mode by background shear is only apparent in mode 4 structure, as a high in the western-central part of the basin. Near the source the upwelling is spread out somewhat, reducing the maximum upward velocity and thus making 
the downwelling elsewhere in the basin easier to see. There is more of a downstream influence, and the temperature can therefore be thought of as being more passive. This agrees with intuition, since vertical shear might be expected to reduce the vertical scale, and the downstream influence of small vertical scale features has been noted.

Other cases (not shown) with different decay scales and background flow strengths were examined. Solutions with shear and strong heating approach those discussed above. Stronger shear (varying both strength and decay scale) solutions are not much different than the one illustrated (Figure 4.10); certain features are more or less emphasized, but all the elements of all the solutions are present to some degree in this case.

The numerical solution with background shear has general features in common with the analytical calculation (Figure 3.12). For comparison to the analytical correction field, the numerical solution (Figure 4.10) ought to be contrasted with the case with background shear (Figure 4.9). An exact correspondence between the numerical and analytical solutions cannot be made because the vertical structure in the numerical model was originally intended to represent an Ekman layer, and is therefore different from that in the analytical calculation. Nevertheless, the common features are that mode 1 gains an influence to the north and downstream, the amplitude of mode 2 is increased slightly in the interior, steepening the pattern, and mode 3 is spread diffusively over a wider area.

\subsection{Discussion}

A satisfying correspondence between the basic behavior of the numerical and analytical models has been found, and suggests that some fundamental physical ideas contained in the simpler conceptual model are relevant when the full equations are solved in a basin. On the whole the important nonlinear interactions occur near the source, generating modes which then affect the rest of the basin according to the value of $\mathrm{C}$, the ratio of phase speed to background flow strength. Judging from the relation between isotherm excursion and heating in the model, the analytic solutions seem to span much of the range of nonlinearity of the numerical solutions. To a greater degree than expected, the effect of nonlinearity is contained in the analytic model. The narrow nonlinear plume was not predicted however, although in retrospect perhaps it could have been.

To gauge the importance of vertical resolution in the numerical model, a study of the ratio of energy in unforced modes to that in forced modes was made. The result was that the forcing $\mathrm{Q} / \mathrm{N}^{2}$ needed to be about an order of magnitude more than the estimated real average value for the sum of the energy in the unforced 


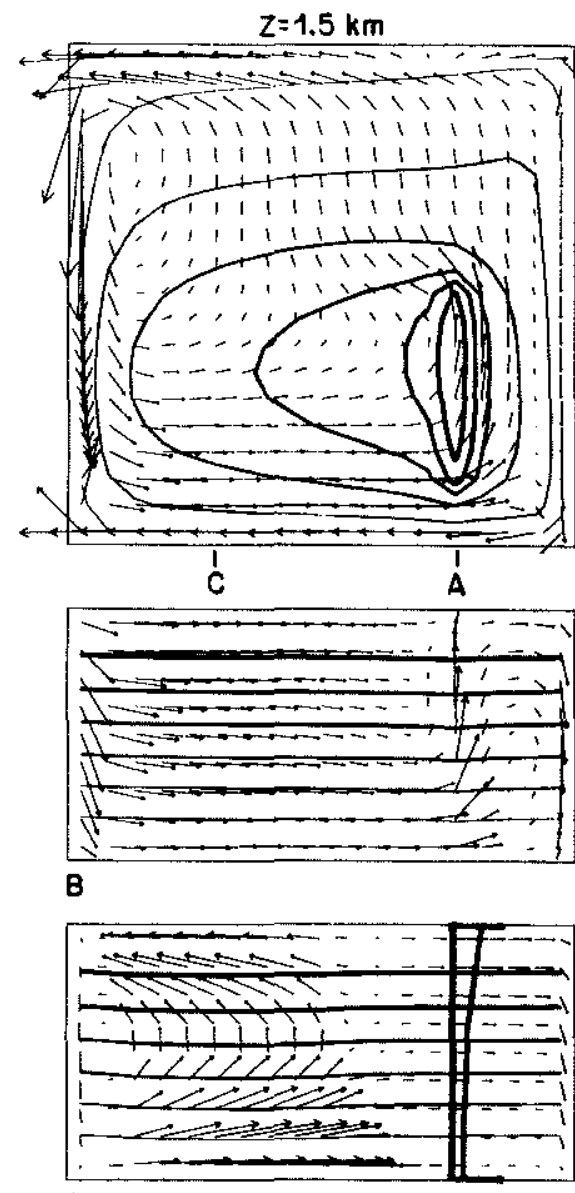

A
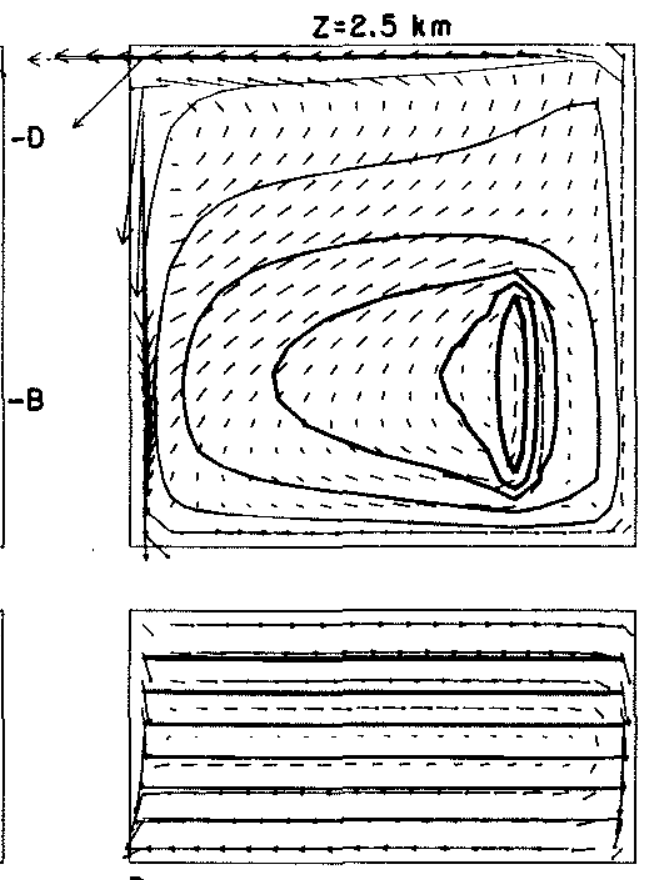

D

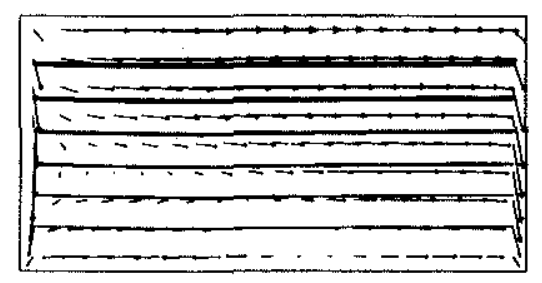

C

Figure 4.10: a) Solution using background flow with vertical shear. The geometry, stratification, and heating are as in $4.9\left(\mathrm{Q}_{s}=1\right)$. The parameter $a \epsilon / L \gamma=3 \times 10^{-2}$, $\mathrm{U} \max =0.1 \mathrm{~cm} / \mathrm{s}, \mathrm{Wmax}=4 \times 10^{-5}, \mathrm{HA} / \mathrm{VA}=4 \times 10^{-2}, \mathrm{Pe}=0.05$. 

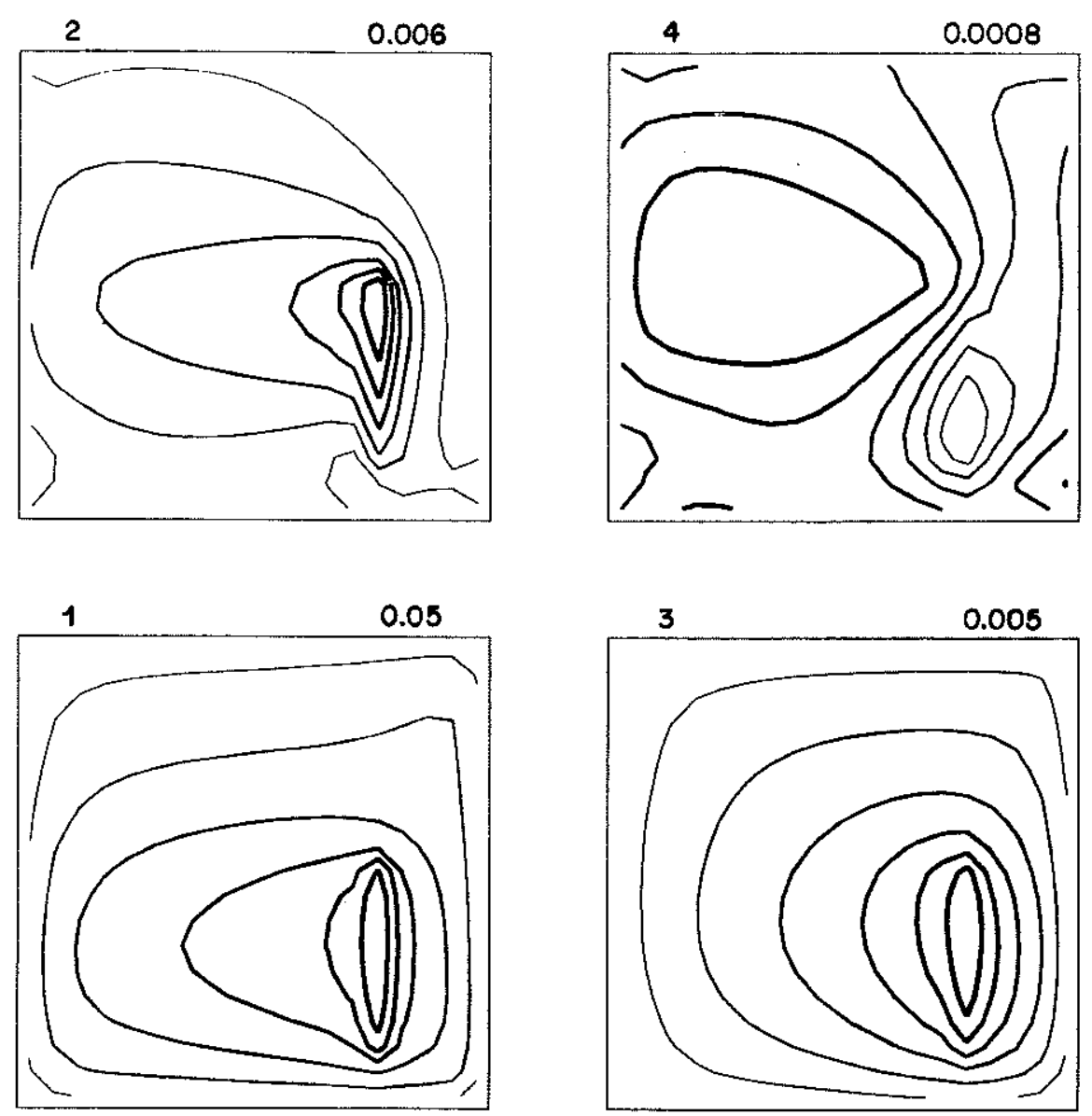

Figure 4.10: b) Mode amplitudes for case with background vertical shear, with maximum values. 
modes to be comparable (greater than a few percent) to that in the forced modes. At these valuess of forcing, which for the weaker stratification case of $\mathrm{N}=1 \times 10^{-3}$ corresponds to $\mathrm{Q}=10$, the response is on the verge of being statically unstable, and therefore unacceptable. The conclusion is that in these steady solutions, the required resolution is simply that which is deemed adequate for the forcing.

The basic assumption in creating the model was the separation between geothermal heating and the processes responsible for the basic stratification. Imposing temperature at the boundaries restricts the conditions in the interior. In these solutions horizontal advection cannot dominate diffusion and still satisfy the temperature boundary condition. The conditions of the model were designed to address geothermal heating in an established abyssal layer, and they still seem appropriate. This study is intermediate between linear dynamics and a full general circulation model with flux boundary conditions, and hopefully illustrates some phenomenon not present in the former and obscured in the latter. It seems necessary and helpful to understand this step before going on to a more complete thermohaline circulation problem. 


\section{Chapter 5}

\section{Final Conclusion}

The heat transfer from the interior of the earth to the ocean is concentrated by mantle convection at mid-ocean ridges, where new crust is formed. The size of the heat flux near the crest of a ridge is estimated to be of order $1 \mathrm{~W} / \mathrm{m}^{2}$, from theoretical models and by simply counting individual heat sources in limited areas. The nature of the heat transfer varies drastically along the crest, involving both conduction and convection, and fluid motion is forced at many length scales. Some of the ingredients of mantle material are dissolved and carried into the ocean by convection, providing tracers of deep circulation. In this thesis, the processes through which geothermal heating along the crest of mid-ocean ridges influences the circulation and characteristics of surrounding water have been studied.

New CTD measurements from the rift valley of the Mid-Atalntic Ridge at $26^{\circ} \mathrm{N}$ were examined for evidence of a geothermal heat source. A cold, fresh plume

was discovered spreading in a layer $300 \mathrm{~m}$ to $450 \mathrm{~m}$ above the bottom. These characteristics were consistent with a mixture of hot spring water with ambient sea water rising to some equilibrium level. A turbulent entrainment model forced by a continuous point source of buoyancy was used to see how such a plume could form, what its penetration height would be, and what its influence on the temperature and salinity fields would be for different background distributions. The background salinity field was found to be crucial for determining the characteristics of the plume. In the Atlantic, the unstable deep background salinity profile gives the plume added buoyancy which forces it to an equilibrium level where it is relatively fresh and cool. Model results agree with observations and a comparison to a Pacific plume is made to emphasize the dependence of the plume anomalies on the background fields.

The prediction of final equilibrium anomalies for quantities other than temperature and salinity, for instance helium or manganese, was not pursued but could be important in the future. Such calculations would help constrain the chemical fluxes from hydrothermal sources, and perhaps provide a test of the entrainment and mixing hypotheses in the model. The fine-structure calculations possible with vertically averaged CTD data could demonstrated the presence of strong mixing and 
the feasibility of smaller-scale turbulence measurements. Such measurements might help constrain the total heat loss at the crest, including components not directly measurable now.

The convergence and entrainment of ambient water into the plume generates a recirculation around the heat source, because of earth's rotation. The horizontal scale of the recirculation depends on the rotation rate and stratification, while the vertical structure is determined by that of the buoyant plume at the center of the vortex. The turbulent plume model could be used to calculate the vertical structure of the vortex. For a typical plume the main result is that the strength of the vortex seems adequate to trap fluid near the source, even in the presence of background flow of several $\mathrm{cm} / \mathrm{s}$. Thus chemical and thermal anomalies may build up near the source. This flow has not been clearly identified with direct current measurements thus far.

Maps of salinity on two isopycnal surfaces were examined for evidence of a large-scale geothermal heating effect. Salinity minima near the ridge were found, whose presence is supported by several different data sets and the oxygen distribution, but not by the silica distribution. The salinity minima are consistent with the geothermal heating of water flowing north along the ridge, and their location is a good place for future observational programs seeking a geothermal influence outside the rift valley of the Mid-Atlantic Ridge. Despite the supporting evidence this interpretation remains questionable because the magnitude of the variations responsible for the minima are close to the level of measurement error. The observations help define areas for future exploration to test the idea of a heating effect, and to search for possible flow through fracture zones.

Tracer measurements in the South Pacific have demonstrated that the middepth flow is more complicated than that of the Stommel and Arons (1960) deep circulation model. A simple model which includes both a heat-forced flow and a background flow was developed to help investigate possible large-scale influence of a heat source. Only the net effect on the density field of smaller-scale convective processes was represented in the large-scale model. That effect was the isopycnal deformation caused by horizontal motion around a heat source. The model results illustrate how a heat source can force density pertubations and therefore vertical shear upstream of the source, depending on the strength of the background flow relative to the long wave phase speed. The tendency for the vertical structure of the solution is to be different from that of the forcing owing to the separation and dispersal of the vertical modes making up the solution. These results affect the interpretation of observations of tracers and vertical shear by showing how these two properties can have very different and unexpected spatial distributions. A solution whose forcing strength and background flow speed are calibrated to observations 
in the South Pacific illustrates these ideas. Although the agreement is only fair it suggests that the basic components of the model may be relevant in this region.

The large-scale model was extended to include neglected nonlinear terms and vertical structure of the background horizontal velocity. Their effect could be described in terms of new sources along dynamical characteristics of the equations. The magnitude of these effects was generally small; the pattern of the solutions changed, but the overall shape and change was predictable. Furthermore it was found that the three vertical mode representation of a solution was usually adequate at large scales.

A numerical model was used to test these results in a more general situation, and with no restriction on the amplitude of the forcing. The basic ideas about mode separation, upstream and downstream influence, the effect of nonlinearity and background structure were all found to be relevant and understandable in the numerical solutions, based on the experience from the analytical model. The conclusion from the analytical extensions and numerical calculations was that the simple concepts from the analytical model adequately describe the solutions overall, and that nonlinearity and background structure are not important at the most basic level. The agreement between the analytical results and numerical results gives increased confidence that the ideas about the influence of a heat source may carry over to the real ocean. 


\section{Appendix A}

\section{Atlantic Stability Distribution and South Atlantic $\theta-S$ variations near the Mid-Atlantic Ridge}

\section{Stability}

Sections of stability were prepared at $45^{\circ} \mathrm{N}, 24^{\circ} \mathrm{N}, 13^{\circ} \mathrm{N}, 11^{\circ} \mathrm{S}$, and $23^{\circ} \mathrm{S}$ (Figures A.1-A.2) to look for an effect of heating on the density field. The definition of stability used here is $-\frac{1}{\rho} \partial \rho / \partial z$ where $\rho$ is the potential density referenced to the local pressure. The derivative enhances vertical structure in the density field, making it easier to visualize. In the $45^{\circ} \mathrm{N}$ section (Figure A.1, western trough) a layer of low stability near $2000 \mathrm{~m}$ depth exists extending from the western boundary east toward the Mid-Atlantic Ridge. This layer is at the right depth and temperature $\left(3.4^{\circ} \mathrm{C}\right)$ to be Labrador Sea Water. It has been described in the context of low potential vorticity, which is proportional to stability times the Coriolis parameter f, by Talley and McCartney (1982). The lowest stability values at about the same level are confined next to the crest of the ridge. At $24^{\circ} \mathrm{N}$, a layer of low stability also exists, but it is deeper, near $3000 \mathrm{~m}$ and $2.5^{\circ} \mathrm{C}$ and decays to the west rather than to the east from the most extreme values near the crest of the ridge. For comparison, the bottom temperature at the crest can be as low as roughly $2.0^{\circ} \mathrm{C}$. A second layer of low stability occurs near $4500 \mathrm{~m}$ depth, with the lowest values at the western boundary. This structure is connected to salty, high oxygen, low nutrients characteristics near the western boundary (Roemmich and Wunsch, 1985), and so represents a water mass layer of northern origin. A section at $36^{\circ} \mathrm{N}$ was made, but there is no coherent mid-depth minimum, so this section is not displayed. At this latitude there is a region of reduced stability above the western flank of the ridge, though, near $2.4^{\circ} \mathrm{C}$. The crest is shallower, near $2.8^{\circ} \mathrm{C}$. At $24^{\circ} \mathrm{N}$ and more so at $36^{\circ} \mathrm{N}$, the low stability feature is no longer a clean extremum, but becomes ragged and messy.

At $13^{\circ} \mathrm{N}$ (Figure A.1) there is a well defined low stability layer near $3200 \mathrm{~m}$ depth, most extreme near the crest. The potential temperature at the level of the minimum is near $2.3^{\circ} \mathrm{C}$, while the bottom temperature at the crest is closer to $1.8^{\circ} \mathrm{C}$. Note that although the region of low stability is above the extremum of the salinity 
anomaly at $24^{\circ} \mathrm{N}$ and $13^{\circ} \mathrm{N}$, the water is still fresh by a least $-.005 \%$.

Zonal sections of stability in the South Atlantic at $11^{\circ} \mathrm{S}$ and $23^{\circ} \mathrm{S}$ both have a well defined low stability layer near $3000 \mathrm{~m}$ depth, similar to that at $13^{\circ} \mathrm{N}$ (Figures A.1 and A.2). The structure is complicated somewhat by a strong maximum near $4000 \mathrm{~m}$ depth associated with the boundary between deep water and bottom water of northern and southern origin, respectively. The stability minimum is most extreme above the crest of the ridge and decays gradually to the west, disappearing at the western boundary. To the east the stability at the level of the minimum increases abrubtly, and no large-scale minimum is present. Some aspects of this overall pattern are also found in a recent numerical simulation of the Atlantic general circulation including deep water formation by M. Cox (G.F.D.L., Princeton University; personal communication). The low stability layer in the western trough at both $11^{\circ} \mathrm{S}$ and $24^{\circ} \mathrm{S}$ is fairly well represented in the numerical model, and is associated with the expected properties of deep water of northern origin, but the model also shows a weak minimum and maximum in the eastern trough (Angola Basin) as well, which does not occur in the observations.

In overview, south of $24^{\circ} \mathrm{N}$ a well defined mid-depth layer of low stability exists, roughly near $3000 \mathrm{~m}$ depth. Its density is near $45.83 \mathrm{~g} / \mathrm{cm}$ and its potential temperature is about $2.4^{\circ} \mathrm{C}$. It extends at least to $32^{\circ} \mathrm{S}$ where it appears in a section of stability presented by Reid, Nowlin, and Patzert (1977) confined to the eastern half of the western trough (their Figure 3b). They did not comment on this particular feature, but did mention that near this depth various extrema in stability may be related to the layering of North Atlantic Deep Water discussed by Wüst. In their view the stability extrema represent distinct water mass layers and the boundaries between them. This view is supported by observations at other latitudes.

Lateral variations in stability could result from flow generated by the heating or by vertical shear of horizontal flow unrelated to heating, making their interpretation ambiguous. In addition, mixing at the crest may not be entirely convective. It is conceivable that mechanical mixing near the crest could generate low stability, if there were eddy flow of sufficient strength to lift water over topographic obstructions of a height comparable to the thickness of the layer, about $500 \mathrm{~m}$. A Froude number kind of argument can be made to estimate the required strength. The flow would have to be such that $U=N \delta z$, where $N$ is the buoyancy frequency, to have enough kinetic energy to overcome gravity. For $\delta z=500 \mathrm{~m}, N=10^{-3} \mathrm{~s}^{-1}, U$ is $50 \mathrm{~cm} / \mathrm{s}$. This value seems excessive in deep water, even near strong topography. Saunders and Francis (1985) used the same arguement to dismiss the ventilation of isolated segments of the median valley at the crest by this mechanism. Although the flow may not be strong enough to go over obstructions, if it is time-dependent there may be occasional overturning in isolated valleys. Dense water could flow into these 
valleys from time to time since vertical excursions of isopycnal surfaces associated with large-scale geostrophic shear in the component of flow along the crest can be several hundred meters, for a vertical shear of order $1 \mathrm{~cm} / \mathrm{s} / \mathrm{km}$. Perhaps some mixing occurs in the complicated adjustment process of flow near isolated valleys along the crest, but as long as such flow is steady no mechanical mixing is expected.

Throughout the depth range $2500 \mathrm{~m}$ to $3500 \mathrm{~m}$ the stability at the TAG site is low, about $5 \times 10^{-9} \mathrm{~cm}^{-1}$. Although any single vent system is not necessarily important on a large-scale, the TAG site at $26^{\circ} \mathrm{N}$ does seem to be an example of a source region of low stability, in addition to relatively fresh water. The density near the equilibrium level of the hydrothermal plume there is about $45.81 \mathrm{~g} / \mathrm{cm}^{3}$, which is above the levels discussed in the text. On this surface, near $2.5^{\circ} \mathrm{C}$, salinity is still low above the ridge, but without showing a minimum. East of the ridge the high salinity Mediteranean overflow water has a stronger influence. This segment of the ridge is open to the interior at fracture zones to the north and south, so the low values are not topographically isolated.

Constant stability on a level surface does not imply flow, but where there are horizontal variations in stability isopycnals are vertically spread apart or brought closer together. Thus the reduced stability towards the crest means an increasing poleward component of flow through the thermal wind relation. One can go further by considering a purely vertical advection-diffusion density equation balance $w \rho_{z}=\kappa \rho_{z z}{ }^{1}$, thought to be valid in the interior (Warren, 1977). Then away from sources a stability extremum must be associated with a level of no vertical motion (still true for variable diffusion coefficient as long as it is a function of $\rho_{z}$ ). Suppose that the bottom is flat. Then according to the geostrophic vorticity relation $\beta v=f w_{z}$ (Chapter 3), which can be vertically integrated to the level of the extremum from the bottom where $w=0$, there would be opposing poleward and equatorward flow underneath. The western boundary current in the South Atlantic has this kind of vertical structure, but the effect of planetary $\beta$ ought to confine it to be in the west. From the observed stability distribution in the two South Atlantic sections and the above balances, and also assuming for simplicity that the vertical diffusion coefficient is constant, the sense of the opposing flow can be inferred. Positive curvature immediately below the stability minimum means that $w$ is negative and hence $w_{z}$ is positive, so that the flow is poleward (south). Below this $w$ has to decrease to zero at the stability maximum and again at the bottom, in either case requiring a flow reversal ( $w_{z}$ changes sign). Thus below the stability minimum horizontal advection of density will eventually dominate vertical advection as $w$ gets small toward the bottom, and then $w$ is no longer necessarily

\footnotetext{
${ }^{1}$ Strictly, potential density is the proper density variable in the advection term, that is, the adiabatic compression effect must be subtracted from the advection term, see Warren (1977).
} 
proportional to the curvature. The flow could therefore remain equatorward to the bottom, because of the tendency for equatorward advection of dense water to make $w$ negative $\left(-\frac{v \rho_{y}}{\rho_{z}}<0\right)$. This simple pattern is not clear in the large-scale horizontal density gradient, which is complicated by several changes of sign across the basin (Fuglister, 1960). According to the Stommel and Arons (1960) deep circulation dynamics, North Atlantic Deep Water ought to flow southward in the southern hemisphere, away from its northern hemisphere source. On the other hand, Antarctic Bottom Water can flow northward, if horizontal advection strongly cools the layer near the bottom of the ocean, since this can be balanced by downwelling and diffusion, implying compression and equatorward flow. Bottom topography could alter this picture by forcing a different interior vertical velocity.

The $\theta-S$ relation at $11^{\circ} \mathrm{S}$ and $23^{\circ} \mathrm{S}$ supports this kind of interior flow (Figure A.3). In the western trough at $23^{\circ} \mathrm{S}$, deep water between $2^{\circ} \mathrm{C}$ and $3^{\circ} \mathrm{C}$ has salinity values like those near the western boundary at $11^{\circ} \mathrm{S}$, suggesting that it came from the north. Bottom water above the western flank has a $\theta-S$ relation which is similar at the two latitudes. Below $2^{\circ} \mathrm{C}$ stations having a comparable relative position on the western flank show only a weak salinity shift of about $0.005 \%$ from $23^{\circ} \mathrm{S}$ to $11^{\circ} \mathrm{S}$. 

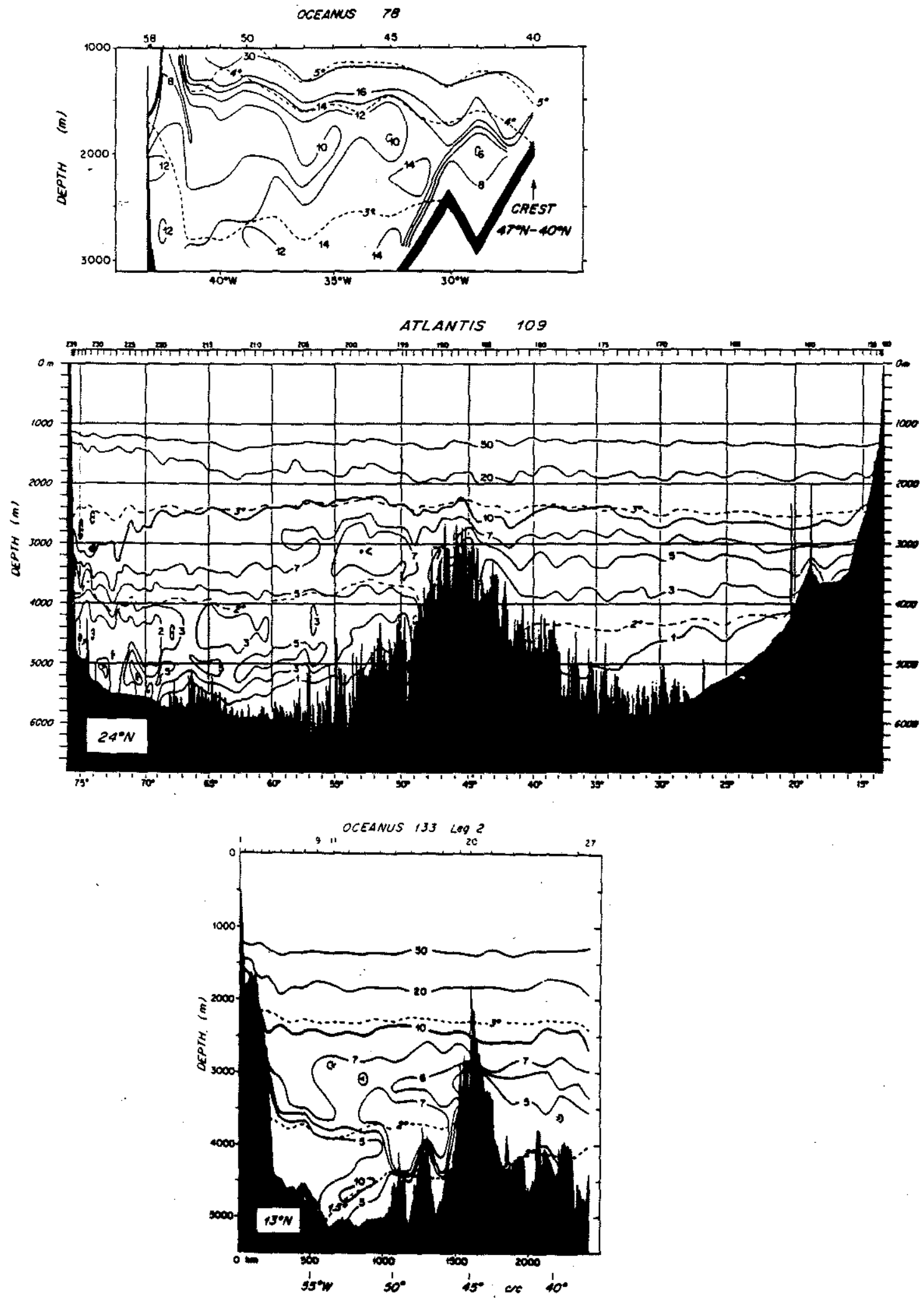

Figure A.1: Stability $\left(\mathrm{cm}^{-1} * 10^{10}\right)$. $45^{\circ} \mathrm{N}$ (upper), $24^{\circ} \mathrm{N}$ (middle), $13^{\circ} \mathrm{N}$ (lower). 
South Atlantic $\theta-S$ variations near the Mid-Atlantic Ridge

In the South Atlantic Broecker and Takahashi (1980) describe some variations of the $\theta-S$ relation near $2.5^{\circ} \mathrm{C}$ using several GEOSECS stations in the western trough. They suggest that in the latitude range $4^{\circ} \mathrm{S}$ to $18^{\circ} \mathrm{S}$ the variation could be caused by a recent release of heat at the ridge crest, although the connection to the ridge is not established. The sections at $11^{\circ} \mathrm{S}$ and $23^{\circ} \mathrm{S}$ allow the connection to be checked. Temperature-salinity plots at selected stations at $11^{\circ} \mathrm{S}$ show anomalies near $2.5^{\circ} \mathrm{C}$ whose magnitude increases toward the crest (Figure A.3). This isotherm lies somewhat above the level of the crest, which is probably closer to $2.3^{\circ} \mathrm{C}$. East of the crest the anomalies disappear and the curve shifts to higher salinities. Vertical diffusion cannot produce such curvature by mixing water above and below; however, the variations could be produced by lateral advection and diffusion along isopycnals from a region where the $\theta-S$ relation is shifted to lower salinity. At $23^{\circ} \mathrm{S}$ the $\theta-S$ relation between $2.0^{\circ} \mathrm{C}$ and $3.0^{\circ} \mathrm{C}$ shifts dramatically across the crest of the ridge. In this temperature range the salinity on the eastern side of the crest is roughly $34.88 \%$, which is fresh enough to be the source of the anomalies at $11^{\circ} \mathrm{S}$.

Supporting evidence that this source of water has the right characteristics for the anomalies is in the oxygen distribution. Associated with fresh variations are low oxygen concentrations (Figure A.3). The oxygen concentrations are consistent with a source region in the eastern trough, where oxygen concentrations are lower (Figure A.3, oxygen at $23^{\circ} \mathrm{S}$ (dash-dot); Bainbridge, 1980). Broecker and Takahashi claim that no other conservative property anomalies were associated with the $\theta-S$ anomalies. Apparently the combination of nitrate and oxygen to form a conservative tracer (their "NO") in the presence of in situ consumption takes away some of the utility of the separate variations of these properties, by canceling out their influence on water in the eastern trough.

These observations suggest the presence of northward flow near $2.5^{\circ} \mathrm{C}$ above the eastern flank of the ridge at $23^{\circ} \mathrm{S}$. Some of this water crosses over the ridge and reaches $11^{\circ} \mathrm{S}$ but not the equator, where the salinity is high (Figure A.3, dots). Northward and westward flow near $2.5^{\circ} \mathrm{C}$ would have to be weak and subject to much horizontal diffusion, since the $\theta-S$ characteristics do change on an isopycnal, becoming warmer and saltier. Northward flow of bottom water above the eastern flank of the Mid-Atlantic Ridge at $23^{\circ} \mathrm{S}$ is expected based on the Stommel and Arons model and the observed interior property variations in bottom water of the Angola Basin (Speer, 1985). This particular flow was not identified because the characteristics of the bottom water above the flank were not consistent from property to property with water of northern or southern origin. The presence of northward flow of deep and bottom water remains speculative. Basically, though, a weak flow near $2.5^{\circ} \mathrm{C}$ from the southern Angola Basin to the Brazil Basin could bring relatively 

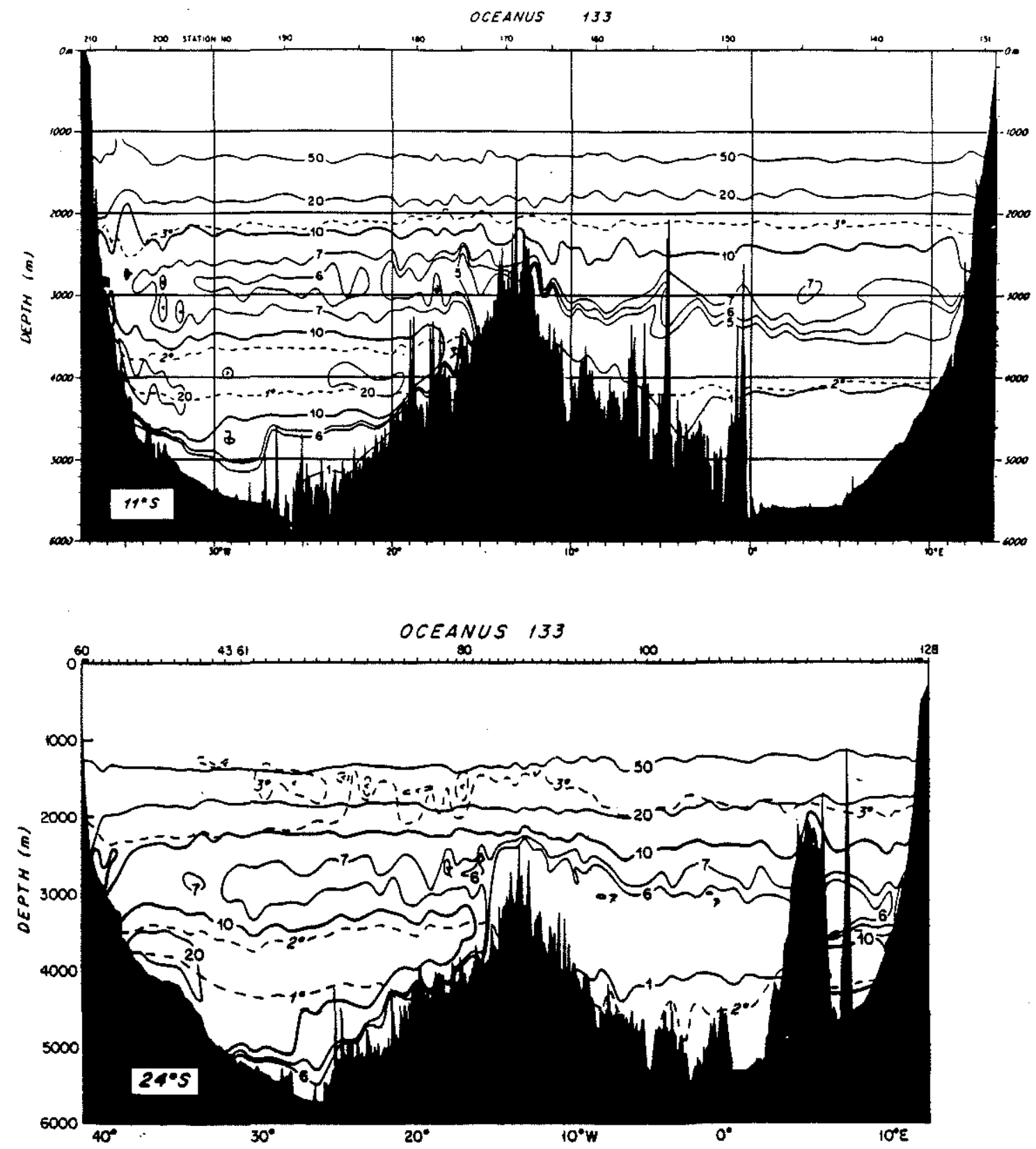

Figure A.2: Stability $\left(\mathrm{cm}^{-1} * 10^{10}\right), 11^{\circ} \mathrm{S}$ (upper), $23^{\circ} \mathrm{S}$ (lower). 
fresh water and account for the observed oxygen and $\theta-S$ variations. The $\theta-S$ variations are strong enough to overwhelm any geothermal signal at slightly lower
temperatures.

No particular variations in the stability profile are associated with the salinity and oxygen variations near $2.5^{\circ} \mathrm{C}$ (Figure A.3). Stability shows a broad decrease with a minimum which does not align with either salinity or oxygen maxima, but North Atlantic Deep Water. Below this layer stability increases to a maximum as the water characteristics change to those of Antarctic Bottom Water. 


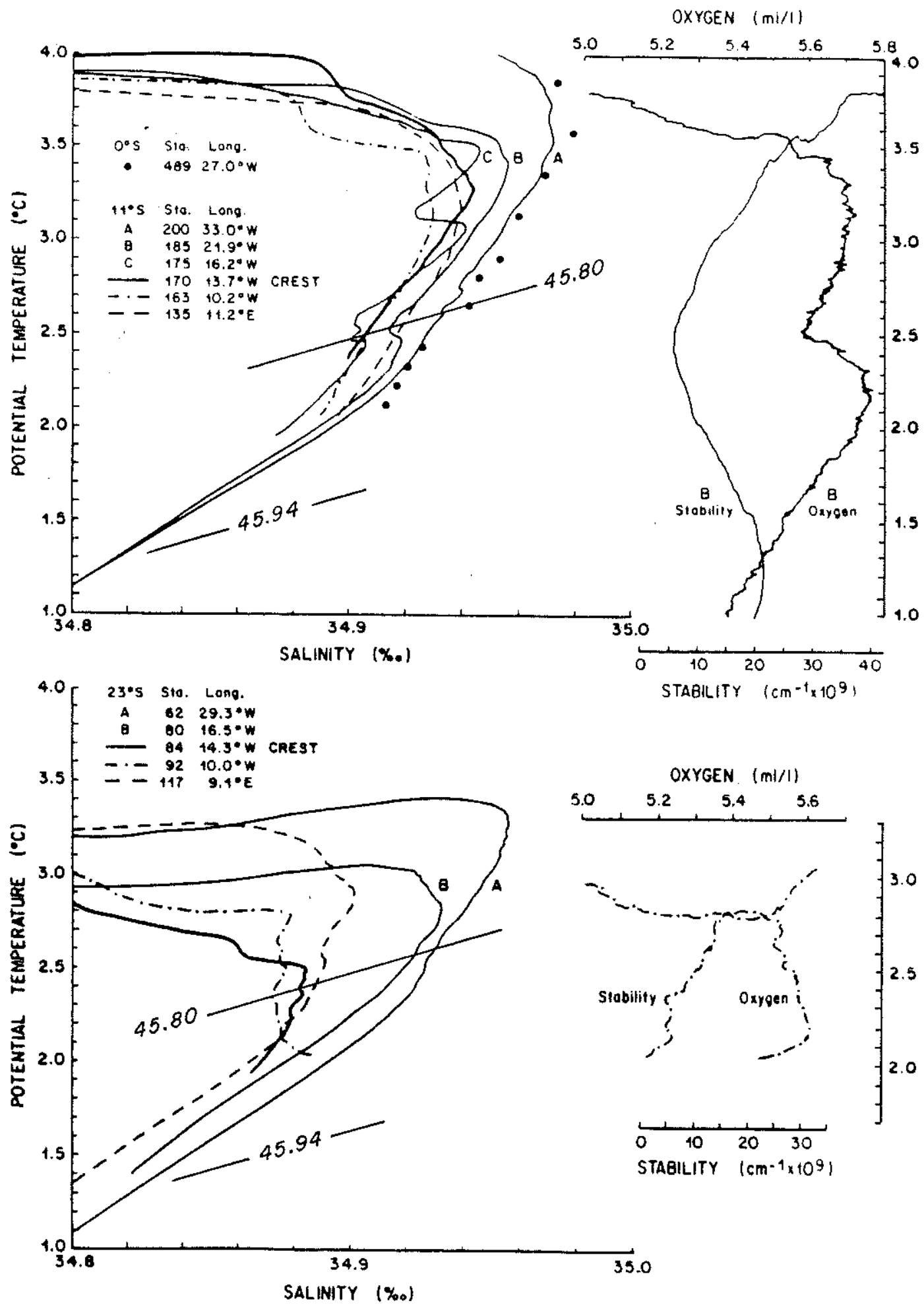

Figure 2.11: Potential temperature versus salinity curves spanning the South Atlantic at $11^{\circ} \mathrm{S}$ (upper) and $23^{\circ} \mathrm{S}$ (lower). Crawford station 489 on the equator is shown for comparison (dots). Stability and oxygen are shown for station 185 at $11^{\circ} \mathrm{S}(\mathrm{B})$ above the western flank of the ridge, and for station 92 at $23^{\circ} \mathrm{S}$. Density is a linear approximation referenced to $4000 \mathrm{db}$. Labels $\mathrm{A}, \mathrm{B}, \mathrm{C}$ go from west to east, the solid line is at the crest. The dash-dot line is mid-way, and the dash line is near to the eastern boundary. 


\section{Appendix B}

\section{Mode Interactions}

For certain ranges of $\mathrm{C}$ and structures of the heat source, relatively simple formal expressions for the correction field can be found. They can be useful for helping to
interpret the interaction of the modes.

Let $\tau, \chi$ be characteristic coordinates such that $\chi=$ constant defines characteristics of a given mode. These coordinates have been given earlier (Chapter 3 ), their form is not important for this discussion. For convenience, $K_{v}=0$, damping can be easily added. The temperature correction $\left(\Theta_{1}\right)$ equation in these coordinates is

$$
\begin{gathered}
\frac{d \Theta_{1}}{d \tau}=\gamma^{+} \cdot S^{+}+\gamma^{-} \cdot S^{-} \\
S^{ \pm}=\nabla_{\tau, \chi} \Theta_{0}^{n \pm 1}
\end{gathered}
$$

where $\gamma^{ \pm}$are the coupling coefficients between the $n \pm 1$ modes and the background structure $\bar{\Theta}^{1}$. They are just the $(A, B),(C, D)$ written in new coordinates.

Two limits can be examined in which the behavior is simplest. If the characteristics for modes $n$ and $n \pm 1$ are parallel then $\Theta_{0}$ does not change along them. Then $S^{ \pm}=\frac{\partial \Theta_{0}}{\partial \chi}$ and so $\Theta_{0}=\Theta_{0}(\chi)$. If the source region is not of too great a meridional extent, $\gamma^{ \pm}=\gamma^{ \pm}(\tau, \bar{\chi})$, where $\bar{\chi}$ is the midpoint of the envelope of characteristics.

or

$$
\frac{d \Theta_{1}}{d \tau}=\gamma^{ \pm}(\tau) S^{ \pm}(\chi)
$$

$$
\Theta_{1}=S^{ \pm}(\chi) \int_{0}^{\tau} \gamma^{ \pm}\left(\tau^{\prime}\right) d \tau^{\prime}
$$

This form still requires integrating along characteristics since the right-hand-side depends on $\tau$, but in since the characteristcs are parallel the transformation to new coordinates can be approximated by simple functions and the integration worked out. Since the coupling coefficients do not change sign in the basin, the magnitude of $\Theta_{1}$ increases monotonically with distance along the characteristic, for fixed characteristic velocity. 
In the other case, if the characteristics are approximately perpendicular (this case can only occur if $\mathrm{C}=\mathrm{O}(1)$ ), then

$$
\frac{\partial}{\partial \chi^{n \pm 1}}=k \frac{\partial}{\partial \tau^{n}}
$$

for some $\mathrm{k}$. If as before the source is localised, $k=\bar{k}(\bar{\tau}, \bar{\chi})$ and $\gamma=\bar{\gamma}(\bar{\tau}, \bar{\chi})$, where $\bar{\tau}, \bar{\chi}$ are the coordinate locations of the intersection of characteristics. Therefore

and so

$$
\frac{d \Theta_{1}^{n}}{d \tau}=\overline{\gamma^{ \pm}} S^{ \pm}=\bar{\gamma}^{ \pm} \frac{\partial \Theta_{0}^{n \pm 1}}{\partial \chi}=\bar{k} \bar{\gamma}^{ \pm} \frac{\partial \Theta_{0}^{n \pm 1}}{\partial \tau}
$$

$$
\Theta_{1}^{n}=\bar{k} \bar{\gamma} \Theta_{0}^{n \pm 1} .
$$

since the barred terms are constants. Now the correction field is just proportional to the mode $n \pm 1 \mathrm{O}(1)$ field. That is, the generation of correction field occuring as the mode $\mathrm{n}$ characteristic enters the envelope of $\mathrm{O}(1)$ characteristics is canceled out as it leaves the envelope.

For certain values of $\mathrm{C}$ it is possible to find the correction accurately for a given mode in this manner by piecing together solutions in different parts of the basin. In general, however, the required range of $\mathrm{C}$ will vary from mode to mode. This method is most useful only as an aid to interpreting the numerical calculation. 


\section{References}

Bainbridge, A.E. (1980) GEOSECS Atlantic Expedition: Volume 2, Sections and Profiles. National Science Foundation, Washington D.C., $196+$ xiv pp.

Baker, E.T., G.J. Massoth, and R.A. Feely (1987) Cataclysmic hydrothermal venting on the Juan de Fuca Ridge. Nature 329:149-151.

Brewer, P. G., J. L. Sarmiento, and W. M. Smethie Jr. (1985) The Transient Tracers in the Ocean (TTO) program: the North Atlantic study, 1981; the tropical Atlantic study, 1983. Journal of Geophysical Research 90:6903-6905.

Broecker, W. S. and T. Takahashi (1980) Hydrography of the central Atlantic-III. The North Atlantic deep-water complex. Deep-Sea Research 27(A):591-613.

Campbell, A. C., and J. M. Gieskes (1984) Water column anomalies associated with hydrothermal acitivity in the Guaymas Basin, Gulf of California. Earth and Planetary Sciences Letters 68:57-72.

Chan, L. -H., D. Drummond, J. M. Edmond, and B. Grant (1977) Barium distribution in the Atlantic GEOSECS stations. Deep-Sea Research 24:613-649.

Charlou, J. L., L. Dmitriev, H. Bougault, and H. D. Needham (1988) Hydrothermal $\mathrm{CH}_{4}$ between $12^{\circ} \mathrm{N}$ and $15^{\circ} \mathrm{N}$ over the Mid-Atlantic Ridge. Deep-Sea Research 35:121-131.

Crane, K. , F. Aikman III, R. Embley, S. Hammond, A. Malahoff, and J. Lupton (1985) The distribution of geothermal fields on the Juan de Fuca Ridge. Journal of Geophysical Research $\underline{90}$ (B1):727-744.

Converse, D. R., H. D. Holland and J. M. Edmond (1984) Flow rates in the axial hot springs of the East Pacific Rise $\left(21^{\circ} \mathrm{N}\right)$ : implications for the heat budget and the formation of massive sulfide deposits. Earth and Planetary Science Letters 69:159-175.

Cox, A., (1973) Plate Tectonics and Geomagnetic Reversals. W. H. Freemand and Co., San Francisco. 702 pp.

Edmond, J.M., K.L. Von Damm, R.E. McDuff, and C.I. Measures (1982) Chemistry of hot springs on the East Pacific Rise and their effluent dispersal. Nature 297:187-191.

Fuglister, F.C. (1960) Atlantic Ocean Atlas of Temperature and Salinity Profiles and Data from the International Geophysical Year of 1957-1958. Woods Hole Oceanographic Institution Atlas Series, 1, 209 pp.

Georgi, D.T., and R.W. Schmitt (1983) Fine and microstructure observations on a hydrographic section from the Azores to the Flemish Cap. Journal of Physical Oceanography 13:632-647. 
Gill, A. E. (1982) Atmosphere-Ocean Dynamics. International Geophysics Series, Volume 30. Academic Press, New York. 662pp.

Jenkins, W. J., P. A. Rona, and J. M. Edmond (1980) Excess ${ }^{3} \mathrm{He}$ in the deep water over the Mid-Atlantic Ridge at $26^{\circ} \mathrm{N}$ : evidence of hydrothermal activity. Earth and Planetary Science Letters $\underline{49}: 39-44$.

Joyce, T. M., and K. G. Speer (1987) Modeling the large-scale influence of geothermal sources on abyssal flow. Journal of Geophysical Research 92 (C3):28432850 .

Joyce, T.M., B.A. Warren, and L.D. Talley (1986) The geothermal heating of the abyssal subarctic Pacific Ocean. Deep-Sea Research 33(8):1003-1015.

Kamenkovich, V.M., A.S. Monin, and A.D. Voorhis (1986) The Polymode Atlas. Woods Hole Oceanographic Institution, Woods Hole, Mass., vi+375 pp.

Klinkhammer, G. and Hudson (1986) Dispersal patterns for hydrothermal plumes in the South Pacific using manganese as a tracer. Earth and Planetary Science Letters 79:241-249.

Klinkhammer, G., P. Rona, M. Greaves, and H. Elderfield (1985) Hydrothermal manganese plumes in the Mid-Atlantic Ridge rift valley. Nature 314:727-731.

Knox, R. A. (1971) On the flow induced in a thermally stratified fluid by a source of heat. Ph.D. thesis, Massachusetts Institute of Technology, Cambridge, Mass., $130 \mathrm{pp}$.

Langseth, M. G., Jr. and R. P. Von Herzen (1971) Heat flow through the floor of the world oceans. In: The Sea, Volume IV, Part I, A.E. Maxwell, editor, Wiley-Interscience, pp. 299-352.

Levitus, S. (1982) Climatological Atlas of the World Ocean. NOAA Professional Paper 13, National Oceanic and Atmospheric Administration, Rockville, Maryland, $173+\mathrm{xv}$ pp.

Little, S.A., K.D. Stolzenbach, and R.P. Von Herzen (1987) Measurements of plume flow from a hydrothermal vent field. Journal of Geophysical Research 92:25872596.

Lupton, J. E. and H. Craig (1981) A major helium-3 source at $15^{\circ} \mathrm{S}$ on the East Pacific Rise. Science 214:13-18.

Lupton, J.E., J.R. Delaney, H.P. Johnson, and M.K. Tivey (1985) Entrainment and vertical transport of deep-ocean water by buoyant hydrothermal plumes. Nature 316:621-623.

Mantyla, A. W. (1987) Standard seawater comparisons updated. Journal of Physical Oceanography 17:543-548. 
Millard, R. C., Jr. (1982) CTD Calibration and data processing techniques at WHOI using the 1978 practical salinity scale. International STD Conference and Workshop sponsored by the Marine Technology Society, San Diego Section, February, 1982, La Jolla, California, 19 pp.

Morgan, P. (1982) Heat flow in rift zones. In: Continental and Oceanic Rifts. Geodynamics Series Volume 8, edited by G. Pálmason. American Geophysical Union, Washington, D. C., 309 pp.

Morton, B. R., G. I. Taylor, and J. S. Turner (1956) Turbulent gravitational convection from maintained and instantaneous sources. Proceedings of the Royal Society, 234:1-23.

Östlund, H. G., H. Craig, W. S. Broecker, and D. Spencer (1987) GEOSECS Atlantic, Pacific, and Indian Ocean Expeditions: Volume 7, Shorebased Data and Graphics. National Science Foundation, Washington, D.C., 200 pp.

Reid, J.L. (1981) On the mid-depth circulation of the world ocean. In: Evolution of Physical Oceanography, Scientific Surveys in Honor of Henry Stommel, B. A. Warren and C. Wunsch, editors; The MIT Press, Cambridge, Massachusetts, pp. $70-111$.

Reid, J.L. (1982) Evidence of an effect of heat flux from the East Pacific Rise upon the characteristics of the mid-depth waters. Geophysical Research Letters 9:381-384.

Reid, J.L. (1986) On the total geostrophic circulation of the South Pacific Ocean: flow patterns, tracers and transports. Progress in Oceanography, 16(1), $61 \mathrm{pp}$.

Reid, J.L., W.D. Nowlin, and W.C. Patzert (1977) On the characteristics and circulation of the southwestern Atlantic Ocean. Journal of Physical Oceanography $\mathbf{7}: 62-91$.

Roemmich, D., and C. Wunsch (1985) Two transatlantic sections: meridional circulation and heat flux in the subtropical North Atlantic Ocean. Deep-Sea Research 32:619-664.

Rona, P.A., G. Klinkhammer, T. A. Nelson, J. H. Trefry, and H. Elderfield (1986) Black smokers, massive sulfides, and vent biota at the Mid-Atlantic Ridge. Nature 321:33-37.

Rona, P.A., and K.G. Speer, An Atlantic hydrothermal plume and its heat transfer: TAG area, Mid-Atlantic Ridge crest near $26^{\circ} \mathrm{N}$. In preparation.

Salmon, R. (1986) A simplified linear ocean circulation theory. Journal of Marine Research 44:695-711.

Saunders, P.M. (1982) Circulation in the eastern North Atlantic. Journal of Marine Research 40(Supplement):641-657. 
Saunders P.M., and T.J.G. Francis (1985) The search for hydrothermal sources on the Mid-Atlantic Ridge. Progress in Oceanography 14:527-536.

Sclater, J.G., C. Jaupart, and D. Galson (1980) The heat flow through the oceanic and continental crust and the heat loss of the earth. Reviews of Geophysics and Space Physics 18(1):269-311.

Speer, K. G. (1985) Property distributions and circulation in the Angola Basin. S.M. thesis, Massachusetts Institute of Technology, Cambridge, Mass. 130 pp.

Stefánsson, V., and S. Björnsson (1982) Physical aspects of hydrothermal systems. In: Continental and Oceanic Rifts. Geodynamics Series vol. 8, edited by G. Pálmason. American Geophysical Union, Washington, D. C., 309pp.

Stommel, H. (1982) Is the South Pacific helium-3 plume dynamically active? Earth and Planetary Science Letters 61:63-67.

Stommel, H., and A. B. Arons (1960) On the abyssal circulation of the world ocean-I. Stationary planetary flow patterns on a sphere. Deep-Sea Research 6: $140-154$.

Talley, L. D. and M. S. McCartney (1982) Distribution and circulation of Labrador Sea Water. Journal of Physical Oceanography, 12:1189-1205.

Turner, J. S. (1973) Buoyancy Effects in Fluids. Cambridge University Press, London, $367 \mathrm{pp}$.

Tziperman E. (1987) The Mediterranean outflow as an example of deep buoyancy driven flow. Journal of Geophysical Research 92(C13):14510-14520.

Van Dover, C. L., P. J. S. Franks, and R. D. Ballard (1987) Prediction of hydrothermal vent locations from distributions of brachyuran crabs. Limnology and Oceanography $\underline{32}(4): 1007-1010$.

Vangriesheim, A. (1980) Antarctic bottom water flow through the Vema Fracture Zone. Oceanologica Acta $\underline{3}: 199-207$.

Warren. B. (1973) Transpacific hydrographic sections at Lats. $43^{\circ} \mathrm{S}$ and $28^{\circ} \mathrm{S}$ : the SCORPIO Expedition-II. Deep water. Deep-Sea Research 20:9-38.

Warren, B. (1977) Shapes of deep density-depth curves. Journal of Physical Oceanography $\mathbf{7}: 338-344$.

Warren, B. (1981) Deep circulation of the world ocean. In: Evolution of Physical Oceanography, Scientific Surveys in Honor of Henry Stommel, B. A. Warren and C. Wunsch, editors; The MIT Press, Cambridge, Massachusetts, pp. 641.

Whitham G.B. (1974) Linear and Nonlinear Waves. Wiley-Interscience, J. Wiley $\&$ Sons, New York, 636 pp. 
Worthington, L. V., and W. G. Metcalf (1961) The relationship between potential temperature and salinity in deep Atlantic water. Rapports et Procès-Verbaux, Conseil-International pour l'Exploration de la Mer, 149:122-128.

Worthington, L. V. and W. R. Wright (1970) North Atlantic Ocean Atlas of Potential Temperature and Salinity in the Deep Water Including Temperature, Salinity and Oxygen Profiles from the Erika Dan Cruise of 1962, Woods Hole Oceanographic Institution Atlas Series 2, 24 pp. and 58 plates. 